\title{
DISSERTATION
}

\section{Fuzzy Probability Distributions}

ausgeführt zum Zwecke der Erlangung des akademischen Grades eines

Doktors der technischen Wissenschaften unter der Leitung von

O. Univ.-Prof. Dipl.-Ing. Dr. techn. Reinhard Viertl

E 107

Institut für Statistik und Wahrscheinlichkeitstheorie

eingereicht an der Technischen Universität Wien

Fakultät für Mathematik und Geoinformation

von

Dipl.-Ing. Wolfgang Trutschnig

Matr. Nr.: 9525888

1100 Wien, Quellenstraße 160-12

Wien, am 3. März 2006

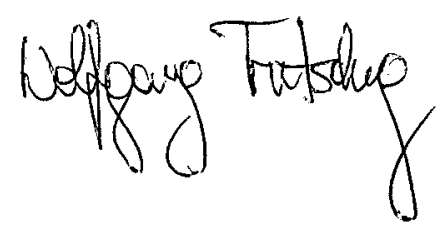




\section{Kurzfassung}

Messwerte kontinuierlicher physikalischer Größen sind a priori unscharf und können mittels des Konzepts der unscharfen Zahlen und unscharfen Vektoren modelliert werden.

Im Sinne einer quantitativen Verarbeitung solcher Daten ist es insbesondere notwendig, das klassische Konzept relativer Häufigkeiten für reelle Stichproben auf sogenannte unscharfe relative Häufigkeiten für unscharfe Stichproben zu erweitern. Die unscharfe relative Häufigkeit einer Menge ist selbst eine unscharfe Zahl. Ausgehend von der Interpretation von Wahrscheinlichkeiten als Grenzwerte relativer Häufigkeiten ist es daher unumgänglich, auch sogenannte unscharfe Wahrscheinlichkeitsverteilungen zu betrachten, für die die Wahrscheinlichkeit eines Ereignisses selbst eine unscharfe Zahl ist.

Nach einer grundlegenden Abhandlung über die wichtigsten algebraischen und topologischen Eigenschaften der Familie der unscharfen Zahlen und der Familie der $d$-dimensionalen unscharfen Vektoren ist daher der Großteil der vorliegende Arbeit der Untersuchung zweier unterschiedlicher natürlicher Zugänge zu unscharfen Wahrscheinlichkeitsverteilungen gewidmet:

Jenem über sogenannte unscharfe Wahrscheinlichkeitsdichten und einem speziellen Integrationsprozess ähnlich dem Aumann-Integral einerseits, und jenem über die Verteilung unscharfer Zufallsvektoren andererseits. Es wird dabei insbesondere versucht, den hohen Grad an Gemeinsamkeit der durch die beiden Zugänge induzierten unscharfen Wahrscheinlichkeitsverteilungen herauszustreichen.

Darüberhinaus wird ein Starkes Gesetz der Großen Zahlen für unscharfe relative Häufigkeiten und unscharfe Wahrscheinlichkeitsverteilungen induziert von unscharfen Zufallsvektoren bezüglich verschiedener Metriken bewiesen und, in Verallgemeinerung reellwertiger stochastischer Prozesse, sogenannte unscharfe stochastische Prozesse definiert, und grundlegende Eigenschaften untersucht. 


\section{Abstract}

The unavoidable imprecision of measurements of continuous physical quantities can be modelled by using the concept of fuzzy numbers and fuzzy vectors. Concerning a quantitative usage of such data it is necessary to extend the classical concept of relative frequencies for real data to so-called fuzzy relative frequencies for fuzzy data. Thereby the fuzzy relative frequency of a set, given a fuzzy sample, is a fuzzy number. Regarding probabilities as limits of relative frequencies it is consequently mandatory to consider so-called fuzzy probability distributions, for which the 'probability' of an event is a fuzzy number.

The present dissertation therefore starts with a chapter about basic algebraic and topological properties of the family of all fuzzy numbers and the family of all $d$-dimensional fuzzy vectors and then mainly concentrates on two different natural approaches to fuzzy probability distributions:

The approach based on so-called fuzzy probability densities and a certain integration process similar to the Aumann-Integral on the one hand, and the approach based on the distribution of a $d$-dimensional fuzzy random vector on the other hand. In particular it is tried to emphasize the high degree of similarity of the fuzzy probability distributions induced by these two different approaches.

In addition a Strong Law of Large Numbers for fuzzy relative frequencies and fuzzy probability distributions induced by fuzzy random vectors with respect to various metrics is proved and basic properties of so-called fuzzy stochastic processes in discrete as well as in continuous time, which are a generalization of classical real-valued stochastic processes, are analyzed. 


\section{Acknowledgement}

Mein aufrichtiger Dank gebührt Prof. Reinhard Viertl, der durch zahlreiche Anregungen und Ideen einen großen Beitrag zu dieser Arbeit geleistet hat.

Ich danke weiters meiner Freundin Nadine für die Liebe und Geduld, sowie meinen Eltern, meiner Schwester und meiner Großmutter für die Unterstützung. 


\section{Contents}

Kurzfassung

Abstract

Acknowledgement

$\begin{array}{lr}\text { Introduction } & 1\end{array}$

1 Fuzzy numbers and fuzzy vectors 4

1.1 Definitions and basic properties . . . . . . . . . . . 4

1.2 Non-empty convex and compact subsets of $\mathbb{R}^{d} \ldots \ldots . \ldots 7$

1.2 .1 Hausdorff metric . . . . . . . . . . . . . . 8

1.2 .2 Support functions . . . . . . . . . . . . . . . 18

1.2.3 $L^{p}$-metrics induced by support functions . . . . . . 27

1.3 Operations on $\mathcal{F}_{c}^{d} \ldots \ldots \ldots \ldots \ldots$

1.4 Support functions for $\mathcal{F}_{c}^{d} \ldots \ldots \ldots \ldots \ldots \ldots$

1.5 Metrics on subclasses of $\mathcal{F}_{c}^{d} \ldots \ldots \ldots 37$

2 Fuzzy probability distributions $\quad 50$

2.1 Motivation . . . . . . . . . . . . . . . . 50

2.2 Discrete fuzzy probability distributions . . . . . . . . . 56

2.3 Fuzzy probability distributions induced by fuzzy probability

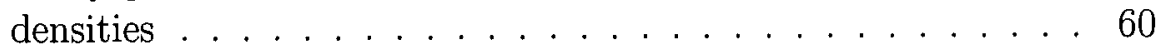

2.4 General fuzzy probability distributions . . . . . . . . . . 80

3 Expectation and other characteristics 82

3.1 Discrete case . . . . . . . . . . . . . . . . . 82

3.2 Case of fuzzy probability distributions induced by fuzzy probability densities . . . . . . . . . . . . . 85

4 Fuzzy random variables and vectors $\quad 91$

4.1 Fuzzy random variables . . . . . . . . . . . . . . . . . 92 
4.2 Fuzzy random vectors . . . . . . . . . . . . . . . . . . . . . 104

4.3 Fuzzy probability distributions induced by fuzzy random variables and fuzzy random vectors . . . . . . . . . . 107

5 Fuzzy stochastic processes $\quad 119$

5.1 Motivation, basic definitions, and

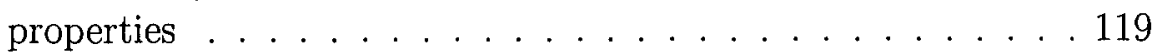

5.2 Independence of fuzzy random vectors . . . . . . . . . . 132

5.3 A Strong Law of Large Numbers for fuzzy relative frequencies 134

6 Appendix 140

6.1 Short introduction to Souslin sets . . . . . . . . . . . . . 140

6.2 Results from functional analysis and measure theory . . . . . 149

$\begin{array}{ll}\text { List of Symbols } & 151\end{array}$

Bibliography 155

$\begin{array}{ll}\text { Curriculum Vitae } & 159\end{array}$ 


\section{Introduction}

As a matter of fact in many real situations uncertainty is not only present in form of randomness (stochastic uncertainty) but also in form of fuzziness (imprecision), for instance due to the inexactness of measurements of continuous quantities. Examples, where imprecision should not be neglected, range from environmental data to measurements of physical quantities even on the macroscopic level.

Randomness is modelled by the concept of random variables, whereas imprecision is best modelled by the concept of fuzzy numbers and fuzzy vectors.

From the probabilistic point of view the unavoidable fuzziness of measurements has amongst others the following far-reaching consequence:

Without doubt one of the most central results of probability theory is the Strong Law of Large Numbers (SLLN), which in particular says, that given a sequence $\left(X_{n}\right)_{n \in \mathbb{N}}$ of identically distributed, pairwise independent, integrable random variables the probability of a measurable event $(X \in B)$ can be regarded as the limit of the relative frequencies of $B$ induced by the sequence $\left(X_{n}\right)_{n \in \mathbb{N}}$ (with probability one).

Incorporating into considerations the fact that a realistic sample of a onedimensional continuous quantity consists of fuzzy numbers, it is first of all necessary to generalize relative frequencies to the case of fuzzy samples, which yields so-called fuzzy relative frequencies. Furthermore keeping in mind the SLLN it is therefore mandatory to consider and analyze fuzzy-valued 'probabilities' as generalization of classical probabilities, which can be seen as the major task of this dissertation.

The developed 'probability' concept will be called fuzzy probability distribution in order to emphasize that it is a fuzzy-valued mapping and to point out that it is also naturally induced by the distribution of so-called fuzzy random variables and fuzzy random vectors.

In short the present thesis analyzes two approaches to fuzzy probability distributions - that based on so-called fuzzy probability densities and a certain integration process on the one hand, and that based on fuzzy random variables and fuzzy random vectors on the other hand. Furthermore it underlines the high degree of similarity of the two (at first sight) very different 
approaches.

In fact the thesis is divided into six chapters:

Chapter 1 can be seen as preparation for the following chapters. It starts with the definitions of fuzzy numbers and fuzzy vectors and provides results about the family $\mathcal{K}_{c}^{d}$ of all non-empty compact convex sets in $\mathbb{R}^{d}$ concerning the Hausdorff metric $\delta_{H}$, support functions, and the $L_{p}$-metrics induced by support functions. After defining basic operations like sum and scalar multiplication for fuzzy numbers and fuzzy vectors, the results on $\mathcal{K}_{c}^{d}$ are generalized to the families $\mathcal{F}_{c}^{1}$ and $\mathcal{F}_{c}^{d}$ of all fuzzy numbers and $d$-dimensional fuzzy vectors respectively. The main properties of the corresponding support functions are presented and metrics on subclasses of $\mathcal{F}_{c}^{d}$ are analyzed.

These metrics are treated in detail (and modified proofs are presented) since in literature a complete survey is hard to find and since the results are interesting in itself.

Chapter 2 begins with a motivation to consider fuzzy-valued 'probabilities', the definition of fuzzy relative frequencies, and the most important properties of fuzzy relative frequencies regarded as fuzzy-valued set functions.

Before concentrating on general fuzzy probability distributions induced by fuzzy probability densities on arbitrary measure spaces $(\Omega, \mathcal{A}, \mu)$ so-called discrete fuzzy probabilities, going back to J. Buckley [6], are treated briefly since they are a special case of the notion developed in Chapter 2.

Based on a useful compactness argument the main properties of fuzzy probability distributions $\mathbb{P}^{\star}$ induced by fuzzy probability densities $f^{\star}$ like monotonicity, sub- and superadditivity and the behaviour under building complements are analyzed. Furthermore the approach is illustrated by a detailed example and some figures. The chapter is concluded by the proposal of a general definition of a fuzzy probability distribution.

The main ideas presented in Chapter 2 are continued in Chapter 3 to generalize the notions of expectation and $k$-th moment to the case of fuzzy probability distributions induced by fuzzy probability densities. Again using a compactness result both the expectation and the $k$-th moment are easily seen to be fuzzy numbers defined via their corresponding $\alpha$-cuts.

Chapter 4 deals with fuzzy random variables and fuzzy random vectors. Using the rich theory of random sets (compare [29]) at first it is proved that eleven different measurability conditions for fuzzy random variables $X^{\star}$, defined on a complete probability space $(\Omega, \mathcal{A}, \mathcal{P})$, are equivalent. Analogous results for fuzzy random vectors are stated. After that it is shown that, similar to fuzzy relative frequencies, every fuzzy random vector and every fuzzy random variable induces a fuzzy probability distribution $\mathbb{P}^{\star}$ according to the general definition stated at the end of Chapter 2 .

The boundaries of the $\alpha$-cuts of this fuzzy probability distribution $\mathbb{P}^{\star}$ are 
furthermore analyzed with respect to regularity and continuity properties of classical probability measures.

In Chapter 5 so-called fuzzy stochastic processes in discrete as well as in continuous time are motivated and considered. The supremum and infimum of families of fuzzy numbers are explained. Based on these definitions measurability results like the measurability of the limes superior and the limes inferior of a fuzzy stochastic process in discrete time and the measurability of the limes superior and the limes inferior of a separable fuzzy stochastic process in continuous time is proved.

After defining independence for fuzzy random variables and fuzzy random vectors Chapter 5 is concluded by a SLLN for fuzzy probability distributions induced by fuzzy random vectors. This result says that given a sequence $\left(X_{n}^{\star}\right)_{n \in \mathbb{N}}$ of identically distributed, pairwise independent $d$-dimensional fuzzy random vectors for every $d$-dimensional Borel set $B$ the relative frequencies $h_{n}^{\star}(B, \omega)$ converge to $\mathbb{P}^{\star}(B)$ with probability one (with respect to various metrics treated in Chapter 1).

Finally the Appendix, Chapter 6, presents a short introduction to the theory of Souslin sets since some the results are needed in Chapter 4.

Furthermore some theorems from functional analysis and measure theory, which are used throughout the dissertation, are stated as reference. 


\section{Chapter 1}

\section{Fuzzy numbers and fuzzy vectors}

\subsection{Definitions and basic properties}

As already mentioned in the introduction the imprecision of measurements of continuous quantities can be modelled by the concept of fuzzy numbers and fuzzy vectors. A general definition of fuzzy numbers and fuzzy vectors and two elementary (but very useful) about the families of $\alpha$-cuts of fuzzy numbers and fuzzy vectors are stated within this first section.

Fuzzy numbers are a generalization of real numbers and are defined and represented by so-called characterizing functions (compare [9], [41]) .

Definition 1.1 $A$ characterizing function $\xi_{x^{\star}}(\cdot)$ of a fuzzy number $x^{\star}$ is a real function with the following properties:

1. $0 \leq \xi_{x^{\star}}(x) \leq 1 \quad \forall x \in \mathbb{R}$

2. $\exists x_{0} \in \mathbb{R}: \xi_{x^{\star}}\left(x_{0}\right)=1$

3. $\forall \alpha \in(0,1]$ the set $\left[\xi_{x^{\star}}\right]_{\alpha}=\left\{x \in \mathbb{R}: \xi_{x^{\star}}(x) \geq \alpha\right\}$, the so-called $\alpha$-cut, is a compact interval.

The set of all fuzzy numbers will be denoted by $\mathcal{F}_{c}^{1}$.

Examples for characterizing functions are classical indicator functions of single points (in which case the corresponding fuzzy number is called crisp) and every indicator function of a compact interval.

In the same manner fuzzy vectors are defined (compare [9], [20]): 
Definition $1.2 A$ vector-characterizing function $\xi_{\underline{x}^{\star}}(\cdot)$ of a d-dimensional fuzzy vector $\underline{x}^{\star}$ is a real-valued function with the following properties:

1. $0 \leq \xi_{\underline{x}^{\star}}(x) \leq 1 \quad \forall x \in \mathbb{R}^{d}$

2. $\exists x_{0} \in \mathbb{R}^{d}: \xi_{\underline{x}^{\star}}\left(x_{0}\right)=1$

3. $\forall \alpha \in(0,1]$ the set $\left[\xi_{\underline{x}^{\star}}\right]_{\alpha}=\left\{x \in \mathbb{R}^{d}: \xi_{\underline{x}^{\star}}(x) \geq \alpha\right\}$, the so-called $\alpha$-cut, is a compact convex set.

The set of all d-dimensional fuzzy vectors will be denoted by $\mathcal{F}_{c}^{d}$ and the set of all d-dimensional non-empty, convex and compact subsets of $\mathbb{R}^{d}$ will be denoted by $\mathcal{K}_{c}^{d}$.

Remark: Since different definitions of fuzzy vectors - with for instance starshaped $\alpha$-cuts - are also used in literature, the notation $\mathcal{F}_{c}^{d}$ is chosen to emphasize compactness and convexity of the $\alpha$-cuts.

As customary fuzzy vectors $\underline{x}^{\star}$ (fuzzy numbers) and their corresponding vector-characterizing functions $\xi_{\underline{x}^{\star}}$ (characterizing functions) will be identified and simply be denoted by $\xi^{\star}$.

Definition 1.3 Let $\xi^{\star} \in \mathcal{F}_{c}^{d}$ be a d-dimensional fuzzy vector, then the set $\operatorname{supp}\left(\xi^{\star}\right)$, defined by

$$
\operatorname{supp}\left(\xi^{\star}\right):=\overline{\bigcup_{\alpha \in(0,1]}\left[\xi^{\star}\right]_{\alpha}}=\overline{\left\{x \in \mathbb{R}^{d}: \xi^{\star}(x)>0\right\}}
$$

is called the support of the fuzzy vector $\xi^{\star}$, whereby $\bar{A}$ denotes the closure of the set $A$ for every $A \subseteq \mathbb{R}^{d}$.

The set of all d-dimensional fuzzy vectors with compact support will be denoted by $\mathcal{F}_{c, c}^{d}$.

The following two simple theorems describe how fuzzy vectors can be reconstructed via their $\alpha$-cuts and how a nested family $\left(B_{\alpha}\right)_{\alpha \in(0,1]}$ in $\mathcal{K}_{c}^{d}$ can be used to define a fuzzy vector:

Theorem 1.4 Let $\xi^{\star} \in \mathcal{F}_{c}^{d}$ be a d-dimensional fuzzy vector, then it follows that

- $\left(\left[\xi^{\star}\right]_{\alpha}\right)_{\alpha \in(0,1]}$ is a nested family in $\mathcal{K}_{c}^{d}$ with $\left[\xi^{\star}\right]_{\alpha} \supseteq\left[\xi^{\star}\right]_{\beta}$ whenever $\alpha \leq \beta$ and $\alpha, \beta \in(0,1]$, fulfilling

$$
\left[\xi^{\star}\right]_{\beta}=\bigcap_{\alpha<\beta}\left[\xi^{\star}\right]_{\alpha} \quad \text { for every } \beta \in(0,1] .
$$


- The fuzzy vector $\xi^{\star}$ can be reconstructed via the $\alpha$-cuts in the following way:

$$
\xi^{\star}(x)=\left\{\begin{array}{ll}
0 & \text { if } x \notin\left[\xi^{\star}\right]_{\alpha} \forall \alpha \in(0,1] \\
\max \left\{\alpha \in(0,1]: x \in\left[\xi^{\star}\right]_{\alpha}\right\} & \text { otherwise }
\end{array}\right\} \forall x \in \mathbb{R}^{d}
$$

Proof: It follows immediately from the definition of the $\alpha$-cuts of a fuzzy vector that $\left[\xi^{\star}\right]_{\alpha} \supseteq\left[\xi^{\star}\right]_{\beta}$ whenever $\alpha \leq \beta, \alpha, \beta \in(0,1]$ and that $\left[\xi^{\star}\right]_{\alpha} \in \mathcal{K}_{c}^{d}$. In order to prove the first assertion it is therefore sufficient to prove that

$$
\left[\xi^{\star}\right]_{\beta} \supseteq \bigcap_{\alpha<\beta}\left[\xi^{\star}\right]_{\alpha}
$$

If $x \in\left[\xi^{\star}\right]_{\alpha}$ for every $\alpha<\beta$ it follows directly from the definition that $\xi^{\star}(x) \geq \alpha$ for all $\alpha<\beta$. Therefore $\xi^{\star}(x)$ can't be smaller than $\beta$, which means that $x \in\left[\xi^{\star}\right]_{\beta}$.

In order to prove the second assertion define a function $\eta: \mathbb{R}^{d} \rightarrow \mathbb{R}$ by

$$
\eta(x):=\max \left\{\alpha \in(0,1]: x \in\left[\xi^{\star}\right]_{\alpha}\right\}
$$

and define the maximum of the empty set to be zero.

First of all notice that $\eta(\cdot)$ is well-defined since due to the already proved property (1.2) the maximum exists.

If $\eta(x)=\alpha_{0}$ it follows that $x \in\left[\xi^{\star}\right]_{\alpha_{0}}$ and therefore $\xi^{\star}(x) \geq \alpha_{0}$.

On the other hand if $\xi^{\star}(x)=\alpha_{0}$ it follows that $x \in\left[\xi^{\star}\right]_{\alpha_{0}}$, which means that $\eta(x) \geq \alpha_{0}$. This completes the proof.

Theorem 1.5 If $\left(B_{\alpha}\right)_{\alpha \in(0,1]}$ is a family in $\mathcal{K}_{c}^{d}$ with $B_{\alpha} \supseteq B_{\beta}$ if $\alpha \leq \beta$, then $\xi^{\star}$, defined by

$$
\xi^{\star}(x)=\left\{\begin{array}{ll}
0 & \text { if } x \notin B_{\alpha} \quad \forall \alpha \in(0,1] \\
\sup \left\{\alpha \in(0,1]: x \in B_{\alpha}\right\} & \text { otherwise }
\end{array}\right\} \forall x \in \mathbb{R}^{d}
$$

is a d-dimensional fuzzy vector called convex hull of the family $\left(B_{\alpha}\right)_{\alpha \in(0,1]}$ with

$$
\left[\xi^{\star}\right]_{\beta}=\bigcap_{\alpha<\beta} B_{\alpha}
$$

Proof: It is obvious that $\xi^{\star}(x) \in[0,1]$ for every $x \in \mathbb{R}^{d}$ and since $B_{1} \in$ $\mathcal{K}_{c}^{d}$ it follows immediately that there exists at least one $x_{0} \in \mathbb{R}^{d}$ such that $\xi^{\star}\left(x_{0}\right)=1$, so the first two assertions of Definition 1.2 are clearly fulfilled.

It remains to show that

$$
\left[\xi^{\star}\right]_{\beta}=\bigcap_{\alpha<\beta} B_{\alpha}
$$


Suppose $x \in\left[\xi^{\star}\right]_{\beta}$ and $\beta \in(0,1]$. It follows that $\xi^{\star}(x) \geq \beta$, which means that

$$
\sup \left\{\alpha \in(0,1]: x \in B_{\alpha}\right\} \geq \beta .
$$

Consequently for every $\alpha$ with $0<\alpha<\beta$ it follows that $x \in B_{\alpha}$.

On the other hand if $x \in B_{\alpha}$ for every $\alpha<\beta$ obviously

$$
\sup \left\{\alpha \in(0,1]: x \in B_{\alpha}\right\} \geq \beta
$$

holds. Therefore $\xi^{\star}(x) \geq \beta$ and $x \in\left[\xi^{\star}\right]_{\beta}$.

Since the collection $\left(B_{\alpha}\right)_{\alpha \in(0,1]}$ surely has the intersection property (which means that every finite subcollection has non-empty intersection), it has non-empty intersection itself. Furthermore because arbitrary intersections of compact and convex subsets of $\mathbb{R}^{d}$ are again compact and convex it follows that

$$
\left[\xi^{\star}\right]_{\beta}=\bigcap_{\alpha<\beta} B_{\alpha} \in \mathcal{K}_{c}^{d}
$$

Using so-called support functions a stronger version of Theorem 1.5 can be proved (compare Theorem 1.36).

Since the $\alpha$-cuts of fuzzy vectors are elements in. $\mathcal{K}_{c}^{d}$ by definition the whole theory of convex bodies in $\mathbb{R}^{d}$ can be applied to fuzzy vectors and fuzzy numbers. Consequently some of the most important and most useful results on convex and compact sets in $\mathbb{R}^{d}$ will be presented in the next section.

\subsection{Non-empty convex and compact subsets of $\mathbb{R}^{d}$}

Throughout the whole thesis the system of all non-empty compact subsets of $\mathbb{R}^{d}$ is denoted by $\mathcal{K}^{d}$. As already mentioned before, the system of all nonempty compact convex subsets of $\mathbb{R}^{d}$ is denoted by $\mathcal{K}_{c}^{d}$.

Moreover $\bar{B}(x, r)$ will denote the closed ball with center $x \in \mathbb{R}^{d}$ and radius $r \in[0, \infty)$ with respect to the Euclidean norm $\|\cdot\|_{2}$.

Definition 1.6 For every set $A \subseteq \mathbb{R}^{d}$, the convex hull conv $(A)$ is the intersection of all convex sets containing $A$.

Since arbitrary intersections of convex sets are convex, the convex hull conv $(A)$ of $A$ itself is a convex set for every $A \subseteq \mathbb{R}^{d}$.

The following theorem is well known: 
Theorem 1.7 (Caratheodory) For every set $A \subseteq \mathbb{R}^{d}$ the convex hull conv $(A)$ is the set of all convex combinations of at most $d+1$ points in $A$, i.e. for every $x \in \operatorname{conv}(A)$ there exist points $a_{1}, a_{2}, \cdots, a_{d+1} \in A$ and real numbers $\gamma_{1}, \gamma_{2}, \cdots, \gamma_{d+1} \in[0,1]$ such that

$$
\sum_{i=1}^{d+1} \gamma_{i}=1 \quad \text { and } \quad x=\sum_{i=1}^{d+1} \gamma_{i} a_{i} .
$$

Proof: Compare for instance [37].

Using Theorem 1.7 it is easy to see that

- $A \subseteq B \subseteq \mathbb{R}^{d}$ implies conv $(A) \subseteq \operatorname{conv}(B)$,

- $A$ bounded implies $\operatorname{conv}(A)$ bounded and

- $A$ compact implies $\operatorname{conv}(A)$ compact.

Definition 1.8 Let $A, B \in \mathcal{K}^{d}$ and $\lambda \in \mathbb{R}$, then the Minkowski sum and the Minkowski scalar multiplication are defined as follows:

$$
A+B:=\{a+b: a \in A, b \in B\}, \quad \lambda A:=\{\lambda a: a \in A\}
$$

It is easy to show that $\mathcal{K}_{c}^{d}$ and $\mathcal{K}^{d}$ are closed under these operations and that $\left(\mathcal{K}_{c}^{d},+\right)$ and $\left(\mathcal{K}^{d},+\right)$ are commutative semigroups.

In fact the following relations hold for $A, B, C \in \mathcal{K}_{c}^{d}$ (or $\mathcal{K}^{d}$ ) and $\lambda_{1}, \lambda_{2} \in \mathbb{R}$ :

$$
\begin{gathered}
(A+B)+C=A+(B+C), \quad A+B=B+A, \\
\lambda(A+B)=\lambda A+\lambda B, \quad \lambda_{1}\left(\lambda_{2} A\right)=\left(\lambda_{1} \lambda_{2}\right) A, \quad 1 A=A
\end{gathered}
$$

It can be proved (compare [34]) that $\left(\mathcal{K}_{c}^{d},+\right)$ can be embedded in a group such that the multiplication with scalars can be extended to this group in such a way, that the resulting system becomes a vector space.

\subsubsection{Hausdorff metric}

Definition 1.9 For $A, B \in \mathcal{K}^{d}$ the Hausdorff metric $\delta_{H}$ is defined by

$$
\delta_{H}(A, B)=\max \left\{\max _{a \in A} \min _{b \in B}\|a-b\|_{2}, \max _{b \in B} \min _{a \in A}\|a-b\|_{2}\right\}
$$

whereby $\|\cdot\|_{2}$ denotes the Euclidean Norm on $\mathbb{R}^{d}$. 
As it is well known (compare [1], [37]) that $\delta_{H}$ satisfies the properties of a metric the proof will be omitted here.

In many cases the following equivalent definition of the Hausdorff metric is useful.

Lemma 1.10 Let $\bar{B}(0,1)$ denote the closed ball in $\mathbb{R}^{d}$ of radius 1 and center 0 with respect to the Euclidean norm and suppose that $A, B \in \mathcal{K}^{d}$, then

$$
\delta_{H}(A, B)=\min \{\lambda \geq 0: A \subseteq B+\lambda \bar{B}(0,1) \text { and } B \subseteq A+\lambda \bar{B}(0,1)\} .
$$

Proof: Because of the compactness of the sets $A, B, \bar{B}(0,1)$ all sets of the form $B+\lambda \bar{B}(0,1)$ and $A+\lambda \bar{B}(0,1)$ are compact for $\lambda \in \mathbb{R}_{0}^{+}$.

If $A \subseteq B+\lambda \bar{B}(0,1)$ for all $\lambda>\mu$, it follows that

$$
A \subseteq \bigcap_{\lambda>\mu}(B+\lambda \bar{B}(0,1))=B+\mu \bar{B}(0,1) .
$$

Interchanging $A$ and $B$ and using the same argument it follows that

$$
\min \{\lambda \geq 0: A \subseteq B+\lambda \bar{B}(0,1) \text { and } B \subseteq A+\lambda \bar{B}(0,1)\}
$$

really exists. Define

$$
\tilde{\delta}(A, B):=\min \{\lambda \geq 0: A \subseteq B+\lambda \bar{B}(0,1) \text { and } B \subseteq A+\lambda \bar{B}(0,1)\}
$$

and suppose that $\delta_{H}(A, B)=\lambda_{1}$ and that $\tilde{\delta}(A, B)=\lambda_{2}$.

Since $\delta_{H}(A, B)=\lambda_{1}$ it follows that $\min _{b \in B}\|a-b\|_{2} \leq \lambda_{1}$ for every $a \in A$. This means that for every $a \in A$ there exists $b \in B$ such that $\|a-b\|_{2} \leq \lambda_{1}$ and consequently $A \subseteq B+\lambda_{1} \bar{B}(0,1)$.

Interchanging $A$ and $B$ and using completely the same argument directly yields $B \subseteq A+\lambda_{1} \bar{B}(0,1)$, which proves that $\lambda_{1} \geq \lambda_{2}$.

On the other hand since $A \subseteq B+\lambda_{2} \bar{B}(0,1)$ and $B \subseteq A+\lambda_{2} \bar{B}(0,1)$ it follows immediately that for every $a \in A$ there exists $b \in B$ such that $\|a-b\|_{2} \leq \lambda_{2}$ and vice versa. Therefore $\lambda_{2} \geq \lambda_{1}$, which completes the proof.

Lemma 1.11 Suppose $\left(A_{n}\right)_{n \in \mathbb{N}}$ is a monotonically decreasing sequence in $\mathcal{K}^{d}$. Then it follows that

$$
\lim _{n \rightarrow \infty} \delta_{H}\left(A_{n}, \bigcap_{i \in \mathbb{N}} A_{i}\right)=0
$$

Proof: Since the sequence $\left(A_{n}\right)_{n \in \mathbb{N}}$ surely has the intersection property it follows that $A:=\bigcap_{i \in \mathbb{N}} A_{i} \in \mathcal{K}^{d}$.

Suppose now that the theorem is wrong. Then there exists $\epsilon>0$ such that 
$\delta_{H}\left(A_{n}, A\right)>\epsilon$ infinitely often. Since obviously $A \subseteq A_{n}$ for all $n \in \mathbb{N}$ it follows that $A_{n} \nsubseteq A+\epsilon \bar{B}(0,1)$ infinitely often and since the sequence $\left(A_{n}\right)_{n \in \mathbb{N}}$ is monotonically decreasing it follows that

$$
A_{n} \nsubseteq A+\epsilon \bar{B}(0,1) \quad \text { for all } n \in \mathbb{N} .
$$

Defining $B_{n}:=A_{n} \backslash \operatorname{int}(A+\epsilon \bar{B}(0,1))$, where int denotes the interior of a set, it follows immediately that $\left(B_{n}\right)_{n \in \mathbb{N}}$ is a decreasing sequence in $\mathcal{K}^{d}$.

Using the same argument as at the beginning of the proof gives

$$
B:=\bigcap_{i \in \mathbb{N}} B_{i} \in \mathcal{K}^{d} \quad \text { and } \quad B \subseteq A
$$

If $x \in A$ then surely $x \in \operatorname{int}(A+\epsilon \bar{B}(0,1))$, and therefore $x \notin B$. This means that $A \cap B=\emptyset$, which is a contradiction to $B \subseteq A$.

Theorem $1.12\left(\mathcal{K}^{\mathrm{d}}, \delta_{H}\right)$ is a complete metric space.

Furthermore if $\left(K_{n}\right)_{n \in \mathbb{N}}$ is a Cauchy sequence in $\mathcal{K}^{d}$ then the limit $K \in \mathcal{K}^{d}$ can be characterized in the following way:

$$
K:=\left\{x \in \mathbb{R}^{d}: \exists\left(x_{n}\right)_{n \in \mathbb{N}} \text { such that } x_{n} \in K_{n} \forall n \text { and } \lim _{n \rightarrow \infty} x_{n}=x\right\}
$$

Proof: If

$$
A_{m}:=\overline{\bigcup_{i=m}^{\infty} K_{i}} \quad \text { and } \quad K:=\bigcap_{m=1}^{\infty} A_{m}
$$

then it follows from the Cauchy property that every $A_{m}$ is bounded and closed and therefore compact. Because of that $\left(A_{m}\right)_{m \in \mathbb{N}}$ is a sequence in $\mathcal{K}^{d}$ that surely has the intersection property as it is decreasing. Hence applying Lemma 1.11 shows that

$$
\lim _{m \rightarrow \infty} \delta_{H}\left(A_{m}, K\right)=0
$$

The next step is to prove that

$$
\lim _{i \rightarrow \infty} \delta_{H}\left(K_{i}, K\right)=0
$$

Let $\epsilon>0$ be arbitrary.

Since $\lim _{m \rightarrow \infty} \delta_{H}\left(A_{m}, K\right)=0$ it follows that there exists $n_{1}=n_{1}(\epsilon) \in \mathbb{N}$ such that for every $m \geq n_{1} A_{m} \subseteq K+\epsilon \bar{B}(0,1)$ holds. This proves that

$$
K_{i} \subseteq K+\epsilon \bar{B}(0,1) \quad \text { for every } i \geq n_{1} .
$$


On the other hand since $\left(K_{n}\right)_{n \in \mathbb{N}}$ is a Cauchy sequence in $\mathcal{K}^{d}$ it follows that there exists $n_{2}=n_{2}(\epsilon) \in \mathbb{N}$ such that for all $i, j \geq n_{2}$

$$
K_{i} \subseteq K_{j}+\epsilon \bar{B}(0,1) \quad \text { and } \quad K_{j} \subseteq K_{i}+\epsilon \bar{B}(0,1) .
$$

Since $K_{j}+\epsilon \bar{B}(0,1)$ is compact it follows that

$$
A_{i} \subseteq K_{j}+\epsilon \bar{B}(0,1) \quad \forall i, j \geq n_{2}
$$

and therefore that

$$
K \subseteq K_{j}+\epsilon \bar{B}(0,1) \quad \forall j \geq n_{2} .
$$

Defining $n_{0}:=\max \left\{n_{1}, n_{2}\right\}$ this shows that $\delta_{H}\left(K_{i}, A\right) \leq \epsilon$ for all $i \geq n_{0}$, which proves (1.5).

It remains to show that $K$ is the set of all limits of sequences $\left(x_{n}\right)_{n \in \mathbb{N}}$ such that $x_{n} \in K_{n}$ for all $n \in \mathbb{N}$. Set

$$
\tilde{K}:=\left\{x \in \mathbb{R}^{d}: \exists\left(x_{n}\right)_{n \in \mathbb{N}} \text { such that } x_{n} \in K_{n} \forall n \text { and } \lim _{n \rightarrow \infty} x_{n}=x\right\} .
$$

Suppose $x \in \tilde{K}$ and $\left(x_{n}\right)_{n \in \mathbb{N}}$ is a sequence converging to $x$ with $x_{n} \in K_{n}$ for all $n \in \mathbb{N}$. In order to prove that $x \in K$ it is sufficient to show that $x \in A_{m}$ for every $m \in \mathbb{N}$ since

$$
K=\bigcap_{m=1}^{\infty} A_{m}=\bigcap_{m=1}^{\infty} \overline{\bigcup_{i=m}^{\infty} K_{i}}
$$

By $\left\{x_{m}, x_{m+1}, \cdots\right\} \subseteq \bigcup_{i=m}^{\infty} K_{i} \subseteq A_{m}$ it follows that $x \in A_{m}$ for every $m \in \mathbb{N}$. This proves that $\tilde{K} \subseteq K$.

On the other hand if $x \in K$ for every $m \in \mathbb{N}$ there exists a sequence $\left(x_{n}^{m}\right)_{n \in \mathbb{N}}$ fulfilling

$$
\lim _{n \rightarrow \infty} x_{n}^{m}=x \quad \text { and } \quad x_{n}^{m} \in \bigcup_{i=m}^{\infty} K_{i} \quad \forall n \in \mathbb{N} .
$$

(In the following $d(\cdot, \cdot)$ denotes the metric induced by the Euclidean norm $\|\cdot\|_{2}$ on $\mathbb{R}^{d}$.)

If $m_{1}=1$ there surely exists $n_{1} \in \mathbb{N}$ such that $d\left(x_{n}^{m_{1}}, x\right) \leq \frac{1}{2}$ for all $n \geq n_{1}$. Furthermore there also exists $j_{1} \in \mathbb{N}$ such that $x_{j_{1}}:=x_{n_{1}}^{m_{1}} \in K_{j_{1}}$.

Setting $m_{2}=j_{1}+1$ there exists $n_{2} \in \mathbb{N}$ such that $d\left(x_{n}^{m_{2}}, x\right) \leq \frac{1}{2^{2}}$ for all $n \geq n_{2}$ and there exists $j_{2} \in \mathbb{N}$ with $j_{2} \geq m_{2}$ and $x_{j_{2}}:=x_{n_{2}}^{m_{2}} \in K_{j_{2}}$.

Proceeding inductively in the same manner gives a strictly increasing sequence $\left(j_{l}\right)_{l \in \mathbb{N}}$ of natural numbers and a sequences $\left(x_{j_{l}}\right)_{l \in \mathbb{N}}$ with

$$
d\left(x_{j_{l}}, x\right) \leq \frac{1}{2^{l}} \quad \text { and } \quad x_{j_{l}} \in K_{j_{l}} \quad \forall l \in \mathbb{N} .
$$


Obviously $\left(x_{j_{l}}\right)_{l \in \mathbb{N}}$ is a Cauchy sequence in $\mathbb{R}^{d}$. Using the Extension Lemma (Lemma 1.13) this sequence can be extended to a Cauchy sequence $\left(\tilde{x}_{j}\right)_{j \in \mathbb{N}}$ fulfilling $\tilde{x}_{j} \in K_{j} \forall j, \tilde{x}_{j_{l}}=x_{j_{l}}$ for all $l \in \mathbb{N}$ and $\lim _{j \rightarrow \infty} x_{j}=x$. This shows that $x \in \tilde{K}$ and completes the proof

The following simple Lemma was used in the proof of Theorem 1.12 and can be found for example in [1].

Lemma 1.13 (Extension Lemma) Suppose $\left(A_{n}\right)_{n \in \mathbb{N}}$ is a Cauchy sequence in $\mathcal{K}^{d}$. Furthermore suppose that $\left(n_{j}\right)_{j \in \mathbb{N}}$ is a strictly monotonically increasing sequence in $\mathbb{N}$ and that $\left(x_{n_{j}}\right)_{j \in \mathbb{N}}$ is a Cauchy sequence in $\left(\mathbb{R}^{d},\|\cdot\|_{2}\right)$ such that $x_{n_{j}} \in A_{n_{j}}$ for all $j \in \mathbb{N}$.

Then there exists a Cauchy sequence $\left(\tilde{x}_{n}\right)_{n \in \mathbb{N}}$ fulfilling $\tilde{x}_{n} \in A_{n}$ for all $n \in \mathbb{N}$ and $\tilde{x}_{n_{j}}=x_{n_{j}}$ for all $j \in \mathbb{N}$.

Proof: As before let $d(\cdot, \cdot)$ denote the metric induced by the Euclidean Norm $\|\cdot\|_{2}$ on $\mathbb{R}^{d}$. For $n \in\left\{1, \cdots, n_{1}\right\}$ choose $\tilde{x}_{n} \in A_{n}$ so that

$$
d\left(\tilde{x}_{n}, x_{n_{1}}\right)=\min _{a \in A_{n}} d\left(x_{n_{1}}, a\right)
$$

which is possible since $A_{n} \in \mathcal{K}^{d}$.

Analogous for every $j \in\{2,3, \cdots\}$ and every $n \in\left\{n_{j}+1, \cdots, n_{j+1}\right\}$ choose $\tilde{x}_{n} \in A_{n}$ so that

$$
d\left(\tilde{x}_{n}, x_{n_{j}}\right)=\min _{a \in A_{n}} d\left(x_{n_{j}}, a\right)
$$

Let $\epsilon>0$ be arbitrary.

Then there exists $N_{1}(\epsilon)$ such that

$$
d\left(x_{n_{j}}, x_{n_{k}}\right)<\frac{\epsilon}{3} \text { for all } n_{j}, n_{k} \geq N_{1} \quad \text { and }
$$

there exists $N_{2}(\epsilon)$ such that

$$
d\left(A_{n}, A_{m}\right)<\frac{\epsilon}{3} \text { for all } n, m \geq N_{2} .
$$

Suppose now that $n, m>\max \left\{N_{1}, N_{2}\right\}$.

Choose $k$ and $j$ so that $m \in\left\{n_{j}+1, \cdots, n_{j+1}\right\}$ and $n \in\left\{n_{k}+1, \cdots, n_{k+1}\right\}$.

Because of $n_{j}, n_{k} \geq \max \left\{N_{1}, N_{2}\right\}$ it follows immediately that

$$
d\left(\tilde{x}_{m}, \tilde{x}_{n}\right) \leq d\left(\tilde{x}_{m}, x_{n_{j}}\right)+d\left(x_{n_{j}}, x_{n_{k}}\right)+d\left(x_{n_{k}}, \tilde{x}_{n}\right) \leq \epsilon
$$

which proves the theorem.

Remark: Theorem 1.12 and Lemma 1.13 especially prove that the limit in the Hausdoff metric is the same as the topological limit. 
Remark: Theorem 1.12 and Lemma 1.13 in fact are valid in more general situations. One can start with an arbitrary metric space $(X, d)$ and look at the set $\mathcal{H}(X)$ of all non-empty compact subsets of $X$. The Hausdorff metric $\delta_{H}$ then is defined using the original metric instead of the Euclidean norm. It can be shown that $\left(\mathcal{H}(X), \delta_{H}\right)$ inherits many important properties like completeness and compactness from $(X, d)$.

These results are for instance very important in the field of Iterated Function Systems, where questions on convergence of the so-called Hutchinson operator are observed (compare for example [1], [23], [39]).

For $\mathcal{K}^{d}$ the following result similar to the famous theorem of Heine-Borel can be proved (compare [37]):

Theorem 1.14 Every bounded and closed set in $\mathcal{K}^{d}$ is compact. Moreover $\left(\mathcal{K}^{d}, \delta_{H}\right)$ is a separable metric space.

Proof: It suffices to prove that every bounded sequence in $\left(\mathcal{K}^{d}, \delta_{H}\right)$ has a convergent subsequence.

For arbitrary $A \in \mathcal{K}^{d}$ and $r \in \mathbb{R}_{0}^{+}$let

$$
B_{\delta_{H}}(A, r):=\left\{K \in \mathcal{K}^{d}: \delta_{H}(A, K)<r\right\}
$$

denote the open ball with respect to the Hausdorff metric $\delta_{H}$ with center $A$ and radius $r$.

Suppose now that $\left(K_{i}\right)_{i \in \mathbb{N}}$ is a bounded sequence in $\left(\mathcal{K}^{d}, \delta_{H}\right)$.

Then (by definition) there exists a positive real number $R \in(0, \infty)$ such that $K_{i} \subseteq B_{\delta_{H}}(\{0\}, R)$ for all $i \in \mathbb{N}$. In particular there exists a closed cube $W$ of edge length $\gamma$ such that $K_{i} \subseteq W$ for all $i \in \mathbb{N}$.

For each $m \in \mathbb{N}$ the cube can be written as a union of $2^{d m}$ closed subcubes of edge length $2^{-m} \gamma$.

For arbitrary $A \in \mathcal{K}^{d}$ let $Q_{m}(A)$ denote the union of all such small cubes that hit $A$. Since for each $m$ the number of subcubes is finite, the sequence $\left(K_{i}\right)_{i \in \mathbb{N}}$ must have a subsequence $\left(K_{i}^{1}\right)_{i \in \mathbb{N}}$ such that $Q_{1}\left(K_{i}^{1}\right)$ is identical for every $i$. Similarly there exists a subsequence $\left(K_{i}^{2}\right)_{i \in \mathbb{N}}$ of the subsequence $\left(K_{i}^{1}\right)_{i \in \mathbb{N}}$ such that $Q_{2}\left(K_{i}^{2}\right)$ is identical for every $i$.

Continuing in this way, a sequence $Q_{m}\left(K_{i}^{m}\right)$ of unions of subcubes (of edge length $2^{-m} \gamma$ for given $m$ ) and for each $m$ a sequence $\left(K_{i}^{m}\right)_{i \in \mathbb{N}}$ is obtained. If $S$ denotes the closed unit ball in $\mathbb{R}^{d}$ then it follows easily from the construction that for fixed $m$

$$
K_{j}^{m} \subseteq K_{i}^{m}+\frac{\gamma}{2^{m}} \sqrt{d} \quad \text { for all } i, j \in \mathbb{N} .
$$


Since $\left(K_{i}^{l}\right)_{i \in \mathbb{N}}$ is a subsequence of $\left(K_{i}^{m}\right)_{i \in \mathbb{N}}$ if $l \geq m$ it therefore follows that

$$
\delta_{H}\left(K_{j}^{l}, K_{i}^{m}\right) \leq \frac{\gamma}{2^{m}} \sqrt{d} \text { for } l \geq m \text { and } \forall i, j \in \mathbb{N} .
$$

Hence the sequence $\left(K_{m}\right)_{m \in \mathbb{N}}$, defined by $K_{m}:=K_{m}^{m}$, is easily seen to be a Cauchy sequence and a subsequence of the original sequence $\left(K_{i}\right)_{i \in \mathbb{N}}$.

This proves the first part of the theorem.

The second part is an easy consequence of the first part: The first part proves that $\left(\mathcal{K}^{d}, \delta_{H}\right)$ is a $\sigma$-compact metric space. Since every compact metric space is separable (in fact even totally bounded) and countable unions of countable sets are countable it immediately follows that $\left(\mathcal{K}^{d}, \delta_{H}\right)$ is separable.

Since $\mathcal{K}_{c}^{d}$ is a subclass of $\mathcal{K}^{d}$ by definition compactness and other properties of subsets of $\mathcal{K}_{c}^{d}$ will follow if it can be proved that $\mathcal{K}_{c}^{d}$ is a closed subset of $\mathcal{K}^{d}$. This is the content of the next lemma.

Lemma $1.15 \mathcal{K}_{c}^{d}$ is a closed subset of $\mathcal{K}^{d}$.

Proof: Suppose $\left(K_{i}\right)_{i \in \mathbb{N}}$ is a sequence in $\mathcal{K}_{c}^{d}$ converging to a set $K \in \mathcal{K}^{d}$.

If $x, y \in K$ according to Theorem 1.12 there exist sequences $\left(x_{n}\right)_{n \in \mathbb{N}}$ and $\left(y_{n}\right)_{n \in \mathbb{N}}$ such that

$$
\lim _{n \rightarrow \infty} x_{n}=x, \quad \lim _{n \rightarrow \infty} y_{n}=y \quad \text { and } \quad x_{n}, y_{n} \in K_{n} \forall n \in \mathbb{N} .
$$

If $\alpha \in[0,1]$ then $z_{i}:=\alpha x_{i}+(1-\alpha) y_{i} \in K_{i}$ holds for all $i \in \mathbb{N}$.

Since every $K_{i}$ is convex by definition and $\lim _{n \rightarrow \infty} z_{n}=\alpha x+(1-\alpha) y$ it follows that $\alpha x+(1-\alpha) y \in K$ which completes the proof.

Proposition $1.16\left(\mathcal{K}_{c}^{d}, \delta_{H}\right)$ is a complete, separable, $\sigma$-compact metric space in which every bounded and closed set is compact.

Proof: The fact that $\left(\mathcal{K}_{c}^{d}, \delta_{H}\right)$ is complete and separable is an immediate consequence of Lemma 1.15. Furthermore if $\left(A_{n}\right)_{n \in \mathbb{N}}$ is a bounded sequence in $\mathcal{K}_{c}^{d}$ then it is also bounded in $\mathcal{K}^{d}$. According to Theorem 1.14 there exists a convergent subsequence $\left(A_{n_{k}}\right)_{k \in \mathbb{N}}$ with limit $A \in \mathcal{K}^{d}$. Since $\mathcal{K}_{c}^{d}$ is closed it follows immediately that $A \in \mathcal{K}_{c}^{d}$ holds, which shows both that every bounded closed set in $\left(\mathcal{K}_{c}^{d}, \delta_{H}\right)$ is compact and that $\left(\mathcal{K}_{c}^{d}, \delta_{H}\right)$ is $\sigma$-compact.

The following lemma will be helpful in Section 1.5.

Lemma 1.17 Suppose that $A, B \in \mathcal{K}^{d}$, then the following inequality holds for the corresponding convex hulls:

$$
\delta_{H}(\operatorname{conv}(A), \operatorname{conv}(B)) \leq \delta_{H}(A, B)
$$


Proof: Since by assumption $A$ and $B$ are non-empty compact sets the corresponding convex hulls conv $(A), \operatorname{conv}(B)$ are elements in $\mathcal{K}_{c}^{d}$.

Set $\delta_{0}:=\delta_{H}(A, B)$ and suppose that $x \in \operatorname{conv}(A)$, then according to Theorem $1.7 x$ is the convex combination of at most $d+1$ points in $A$, i.e. there exist points $a_{1}, a_{2}, \cdots, a_{d+1} \in A$ and real numbers $\gamma_{1}, \gamma_{2}, \cdots, \gamma_{d+1} \in[0,1]$, such that

$$
\sum_{i=1}^{d+1} \gamma_{i}=1 \quad \text { and } \quad x=\sum_{i=1}^{d+1} \gamma_{i} a_{i} .
$$

It follows immediately from the definition of the Hausdorff metric that for every $a_{i}$ there exists $b_{i} \in B$ such that $\left\|a_{i}-b_{i}\right\|_{2} \leq \delta_{0}$ for all $i \in\{1, \cdots, d+1\}$. For the point $y$, defined by $y=\sum_{i=1}^{d+1} \gamma_{i} b_{i}$ it follows that $y \in \operatorname{conv}(B)$ and

$$
\|x-y\|_{2}=\left\|\sum_{i=1}^{d+1} \gamma_{i}\left(a_{i}-b_{i}\right)\right\|_{2} \leq \sum_{i=1}^{d+1} \gamma_{i} \delta_{0}=\delta_{0} .
$$

Starting with arbitrary $x \in \operatorname{conv}(B)$ and following the same idea finally yields $\delta_{H}(\operatorname{conv}(A), \operatorname{conv}(B)) \leq \delta_{0}$.

Before continuing with so-called support functions an interesting and useful result (Theorem 1.20) about generators of the Borel $\sigma$-algebra $\mathcal{B}\left(\left(\mathcal{K}^{d}, \delta_{H}\right)\right)$ in $\mathcal{K}^{d}$ generated by the Hausdorff metric $\delta_{H}$ will be proved (compare [29] for a more general setting). The following lemma provides the main tool for the proof.

Lemma 1.18 Suppose that $\mathcal{O}_{\delta_{H}}$ denotes the topology induced by the Hausdorff metric on $\mathcal{K}^{d}$ and that $\mathfrak{B}$ denotes the countable basis for the Euclidean topology on $\mathbb{R}^{d}$ consisting of all open balls $B(m, r)$ with $m \in \mathbb{Q}^{d}$ and $r \in \mathbb{Q}^{+}$. Then the following assertions hold:

1. For every open set $G \subseteq \mathbb{R}^{d}$ both sets $\mathcal{K}^{G}:=\left\{K \in \mathcal{K}^{d}: K \subseteq G\right\}$ and $\mathcal{K}_{G}:=\left\{K \in \mathcal{K}^{d}: K \cap \bar{G} \neq \emptyset\right\}$ are open in $\left(\mathcal{K}^{d}, \delta_{H}\right)$, i.e. $\mathcal{K}^{G}, \overline{\mathcal{K}}_{G} \in \mathcal{O}_{\delta_{H}}$ holds.

2. For every non-empty compact set $K_{0} \in \mathcal{K}^{d}$ and every $\epsilon>0$ there exist open balls $B_{1}, B_{2}, \cdots, B_{n} \in \mathfrak{B}$ such that the set $\mathcal{K}_{B_{1}, B_{2}, \cdots, B_{n}}^{\bigcup_{n}^{n} B_{i}}$, defined by $\mathcal{K}_{B_{1}, B_{2}, \cdots, B_{n}}^{\bigcup_{i=1}^{n} B_{i}}:=\left\{K \in \mathcal{K}^{d}: K \subseteq \bigcup_{i=1}^{n} B_{i}, K \cap B_{i} \neq \emptyset \forall i \in\{1,2, \cdots, n\}\right\}$, fulfills $K_{0} \in \mathcal{K}_{B_{1}, B_{2}, \cdots, B_{n}}^{\bigcup_{i=1}^{n} B_{i}} \subseteq B_{\delta_{H}}\left(K_{0}, \epsilon\right):=\left\{K \in \mathcal{K}^{d}: \delta_{H}\left(K_{0}, K\right)<\epsilon\right\}$. 
Proof: In order to prove the first assertion assume that $G \subseteq \mathbb{R}^{d}$ is open and that $L \in \mathcal{K}^{G}$. Since $L$ is compact and $G^{c}$ is closed it follows immediately that

$$
\epsilon:=\inf _{x \in L, y \in G^{c}}\|x-y\|_{2}>0 .
$$

If $K \in B_{\delta_{H}}\left(L, \frac{\epsilon}{2}\right)$ then the definition of the Hausdorff metric (1.4) implies that for every $y \in K$ there exists $x \in L$ fulfilling $\|x-y\|_{2}<\frac{\epsilon}{2}$. Consequently $y \in G$ holds, which shows that $K \subseteq G$. Therefore it follows immediately that $L \in B_{\delta_{H}}\left(L, \frac{\epsilon}{2}\right) \subseteq \mathcal{K}^{G}$, which proves that every $L \in \mathcal{K}^{G}$ is an inner point of $\mathcal{K}^{G}$. Hence $\mathcal{K}^{G}$ is open in $\left(\mathcal{K}^{d}, \delta_{H}\right)$.

If $G \subseteq \mathbb{R}^{d}$ is open and $L \cap G \neq \emptyset$, then there exists a point $x \in L$ and $\epsilon>0$ such that $B(x, \epsilon):=\left\{z \in \mathbb{R}^{d}:\|x-z\|_{2}<\epsilon\right\} \subseteq G$. For every set $K \in B_{\delta_{H}}(L, \epsilon)$ there exists a point $y \in K$ such that $\|x-y\|_{2}<\epsilon$ holds, which implies that $\emptyset \neq B(x, \epsilon) \cap K \subseteq G \cap K$. Consequently $L$ is an inner point of $\mathcal{K}_{G}$, which completes the proof that $\mathcal{K}_{G}$ is open since $L \in \mathcal{K}_{G}$ was arbitrary.

In order to prove the second assertion of the lemma suppose that $K_{0} \in \mathcal{K}^{d}$ and $\epsilon>0$. Choose $\epsilon^{\prime} \in(0, \epsilon) \cap \mathbb{Q}$ and cover $K_{0}$ by finitely many open balls $B_{1}, B_{2}, \cdots, B_{n} \in \mathfrak{B}$ of radius $\frac{\epsilon^{\prime}}{2}$ from the basis $\mathfrak{B}$ that have non-empty intersection with $K_{0}$ (this is possible since $K_{0}$ is compact). Consider the set

$$
\mathcal{K}_{B_{1}, B_{2}, \cdots, B_{n}}^{\bigcup_{n}^{n} B_{i}}:=\left\{K \in \mathcal{K}^{d}: K \subseteq \bigcup_{i=1}^{n} B_{i}, K \cap B_{i} \neq \emptyset \forall i \in\{1,2, \cdots, n\}\right\} .
$$

It follows immediately from the first part of the proof that $\mathcal{K}_{B_{1}, B_{2}, \cdots, B_{n}}^{\bigcup_{n}^{n} B_{i}}$ is as finite non-empty intersection of open sets itself open.

It will be proved now that $K_{0} \in \mathcal{K}_{B_{1}, B_{2}, \cdots, B_{n}}^{\bigcup_{i}^{n} B_{i}} \subseteq B_{\delta_{H}}\left(K_{0}, \epsilon\right)$ holds:

Suppose that $K \in \mathcal{K}_{B_{1}, B_{2}, \cdots, B_{n}}^{\bigcup_{i=1}^{n} B_{i}}$ is arbitrary but fixed. Then for every point $x \in K_{0}$ there exists an index $i_{0} \in\{1,2, \cdots, n\}$ such that $x \in B_{i_{0}}$ is fulfilled. Since $K \cap B_{i_{0}} \neq \emptyset$ holds there exists a point $y \in K$ such that $\|x-y\|_{2}<\epsilon^{\prime}$, which shows that $\max _{x \in K_{0}} \min _{y \in K}\|x-y\|<\epsilon^{\prime}$.

Interchanging $K_{0}$ and $K$ and following the same argumentation implies that $\max _{x \in K} \min _{y \in K_{0}}\|x-y\|<\epsilon^{\prime}$. Consequently by the very definition of the Hausdorff metric (1.4) $\delta_{H}\left(K, K_{0}\right)<\epsilon^{\prime}<\epsilon$ follows, which completes the proof of the lemma.

Proposition 1.19 With the notation used in Lemma 1.18 the family $\mathcal{C}$, defined by

$$
\mathcal{C}:=\left\{\mathcal{K}_{B_{1}, B_{2}, \cdots, B_{n}}^{\bigcup_{n}^{n} B_{i}}: n \in \mathbb{N}, B_{1}, B_{2}, \cdots, B_{n} \in \mathfrak{B}\right\}
$$

is a countable basis for the topology $\mathcal{O}_{\delta_{H}}$. 
Proof: Since $\mathfrak{B}$ is countable the set of all finite subsets of $\mathfrak{B}$ itself is countable, which immediately implies that the family $\mathcal{C}$ is countable. The fact that $\mathcal{C}$ is a basis for the topology is an obvious consequence of Lemma 1.18.

Theorem 1.20 Denote by $\mathcal{B}\left(\left(\mathcal{K}^{d}, \delta_{H}\right)\right)$ the Borel $\sigma$-algebra generated by the Hausdorff metric $\delta_{H}$ on $\mathcal{K}^{d}$ and define

$$
\begin{aligned}
& \mathscr{E}_{1}^{d}:=\left\{\mathcal{K}^{G}=\left\{K \in \mathcal{K}^{d}: K \subseteq G\right\}: G \subseteq \mathbb{R}^{d}, G \text { open }\right\} \\
& \mathscr{E}_{2}^{d}:=\left\{\mathcal{K}_{G}=\left\{K \in \mathcal{K}^{d}: K \cap G \neq \emptyset\right\}: G \subseteq \mathbb{R}^{d}, G \text { open }\right\} .
\end{aligned}
$$

Then both systems $\mathscr{E}_{1}^{d}$ and $\mathscr{E}_{2}^{d}$ generate the Borel $\sigma$-algebra $\mathcal{B}\left(\left(\mathcal{K}^{d}, \delta_{H}\right)\right)$, i.e. $\mathcal{A}_{\sigma}\left(\mathscr{E}_{1}^{d}\right)=\mathcal{A}_{\sigma}\left(\mathscr{E}_{2}^{d}\right)=\mathcal{B}\left(\left(\mathcal{K}^{d}, \delta_{H}\right)\right)$ holds

Proof: The theorem will be proved in two steps - first of all it will be shown that $\mathscr{E}_{1}^{d}$ and $\mathscr{E}_{2}^{d}$ generate the same $\sigma$-algebra and after that it will be proved that this $\sigma$-algebra coincides with $\mathcal{B}\left(\left(\mathcal{K}^{d}, \delta_{H}\right)\right)$.

Suppose that $G$ is an open subset of $\mathbb{R}^{d}$. Approximate $G$ by a sequence $\left(F_{n}\right)_{n \in \mathbb{N}}$ of closed subsets, defined by

$$
F_{n}:=\left\{x \in \mathbb{R}^{d}: \inf _{y \in G^{c}}\|x-y\|_{2} \geq \frac{1}{n}\right\} .
$$

Then it follows immediately that $\bigcup_{n=1}^{\infty} F_{n}=G$, which implies that

$$
\begin{aligned}
\left\{K \in \mathcal{K}^{d}: K \cap G \neq \emptyset\right\}^{c} & =\left\{K \in \mathcal{K}^{d}: K \cap \bigcup_{n=1}^{\infty} F_{n}=\emptyset\right\} \\
& =\bigcap_{n=1}^{\infty}\left\{K \in \mathcal{K}^{d}: K \cap F_{n}=\emptyset\right\} \\
& =\bigcap_{n=1}^{\infty} \underbrace{\left\{K \in \mathcal{K}^{d}: K \subseteq F_{n}^{c}\right\}}_{\in \mathscr{E}_{1}^{d}} \in \mathcal{A}_{\sigma}\left(\mathscr{E}_{1}^{d}\right) .
\end{aligned}
$$

Consequently $\mathscr{E}_{2}^{d} \subseteq \mathcal{A}_{\sigma}\left(\mathscr{E}_{1}^{d}\right)$ and therefore $\mathcal{A}_{\sigma}\left(\mathscr{E}_{2}^{d}\right) \subseteq \mathcal{A}_{\sigma}\left(\mathscr{E}_{1}^{d}\right)$ holds

On the other hand $G$ can also be approximated by a sequence $\left(E_{n}\right)_{n \in \mathbb{N}}$ of open subsets, defined by

$$
E_{n}:=\left\{x \in \mathbb{R}^{d}: \inf _{y \in G^{c}}\|x-y\|_{2}>\frac{1}{n}\right\}
$$

It follows immediately that $\bigcup_{n=1}^{\infty} E_{n}=G$ is satisfied. Furthermore since $E_{n}^{c}$ is closed for every $n \in \mathbb{N}$ the following equality holds:

$$
\left\{K \in \mathcal{K}^{d}: K \subseteq G\right\}^{c}=\left\{K \in \mathcal{K}^{d}: K \cap G^{c} \neq \emptyset\right\}
$$




$$
\begin{aligned}
& =\left\{K \in \mathcal{K}^{d}: K \cap \bigcap_{n=1}^{\infty} E_{n}^{c} \neq \emptyset\right\} \\
& =\bigcap_{n=1}^{\infty} \underbrace{\left\{K \in \mathcal{K}^{d}: K \cap E_{n}^{c} \neq \emptyset\right\}}_{\in \mathscr{E}_{2}^{d}} \in \mathcal{A}_{\sigma}\left(\mathscr{E}_{2} d\right) .
\end{aligned}
$$

Consequently $\mathscr{E}_{1}^{d} \subseteq \mathcal{A}_{\sigma}\left(\mathscr{E}_{2}^{d}\right)$ and therefore $\mathcal{A}_{\sigma}\left(\mathscr{E}_{1}^{d}\right) \subseteq \mathcal{A}_{\sigma}\left(\mathscr{E}_{2}^{d}\right)$ follows. This completes the proof that $\mathcal{A}_{\sigma}\left(\mathscr{E}_{1}^{d}\right)=\mathcal{A}_{\sigma}\left(\mathscr{E}_{2}^{d}\right)$, which in turn especially shows that $\mathcal{A}_{\sigma}\left(\mathscr{E}_{1}^{d}\right)=\mathcal{A}_{\sigma}\left(\mathscr{E}_{2}^{d}\right)=\mathcal{A}_{\sigma}\left(\mathscr{E}_{1}^{d} \cup \mathscr{E}_{2}^{d}\right)$.

The Borel $\sigma$-algebra $\mathcal{B}\left(\left(\mathcal{K}^{d}, \delta_{H}\right)\right)$ is generated by the topology $\mathcal{O}_{\delta_{H}}$ by definition. According to Proposition 1.19 this topology has a countable base $\mathcal{C}$, which consists of sets $\mathcal{K}_{B_{1}, B_{2}, \cdots, B_{n}}^{\cup_{i}^{n} B_{i}}$ that obviously lie in $\mathcal{A}_{\sigma}\left(\mathscr{E}_{1}^{d} \cup \mathscr{E}_{2}^{d}\right)$. Consequently it follows immediately that $\mathcal{O}_{\delta_{H}} \subseteq \mathcal{A}_{\sigma}\left(\mathscr{E}_{1}^{d} \cup \mathscr{E}_{2}^{d}\right)$ holds, which in turn implies that $\mathcal{B}\left(\left(\mathcal{K}^{d}, \delta_{H}\right)\right) \subseteq \mathcal{A}_{\sigma}\left(\mathscr{E}_{1}^{d} \cup \mathscr{E}_{2}^{d}\right)$ holds.

On the other hand since both the family $\mathscr{E}_{1}^{d}$ and the family $\mathscr{E}_{2}^{d}$ according to Lemma 1.18 consist of open sets it follows that $\mathcal{A}_{\sigma}\left(\mathscr{E}_{1}^{d} \cup \mathscr{E}_{2}^{d}\right) \subseteq \mathcal{B}\left(\left(\mathcal{K}^{d}, \delta_{H}\right)\right)$. Finally applying the first part of the proof yields $\mathcal{A}_{\sigma}\left(\mathscr{E}_{1}^{d}\right)=\mathcal{A}_{\sigma}\left(\mathscr{E}_{2}^{d}\right)=$ $\mathcal{B}\left(\left(\mathcal{K}^{d}, \delta_{H}\right)\right)$, which completes the proof.

Not surprisingly an analogous result holds for $\mathcal{K}_{c}^{d}$ instead of $\mathcal{K}^{d}$.

Proposition 1.21 Denote by $\mathcal{B}\left(\left(\mathcal{K}_{c}^{d}, \delta_{H}\right)\right)$ the Borel $\sigma$-algebra generated by the Hausdorff metric $\delta_{H}$ on $\mathcal{K}_{c}^{d}$ and define

$$
\begin{aligned}
& \mathscr{E}_{1, c}^{d}:=\left\{\mathcal{K}^{G}=\left\{K \in \mathcal{K}_{c}^{d}: K \subseteq G\right\}: G \subseteq \mathbb{R}^{d}, G \text { open }\right\} \\
& \mathscr{E}_{2, c}^{d d}:=\left\{\mathcal{K}_{G}=\left\{K \in \mathcal{K}_{c}^{d}: K \cap G \neq \emptyset\right\}: G \subseteq \mathbb{R}^{d}, G \text { open }\right\}
\end{aligned}
$$

Then both systems $\mathscr{E}_{1, c}^{d}$ and $\mathscr{E}_{2, c}^{d}$ generate the Borel $\sigma$-algebra $\mathcal{B}\left(\left(\mathcal{K}_{c}^{d}, \delta_{H}\right)\right)$, i.e. $\mathcal{A}_{\sigma}\left(\mathscr{E}_{1, c}^{d}\right)=\mathcal{A}_{\sigma}\left(\mathscr{E}_{2, c}^{d}\right)=\mathcal{B}\left(\left(\mathcal{K}_{c}^{d}, \delta_{H}\right)\right)$ holds.

Proof: First of all it follows immediately from the definitions (1.9) and (1.10) that both equalities $\mathscr{E}_{1, c}^{d}=\mathscr{E}_{1}^{d} \cap \mathcal{K}_{c}^{d}$ and $\mathscr{E}_{2, c}^{d}=\mathscr{E}_{2}^{d} \cap \mathcal{K}_{c}^{d}$ are satisfied. Furthermore it is well known from measure theory (compare [13]) that $\mathcal{A}_{\sigma}\left(\mathscr{E}_{1}^{d} \cap \mathcal{K}_{c}^{d}\right)=$ $\mathcal{A}_{\sigma}\left(\mathscr{E}_{1}^{d}\right) \cap \mathcal{K}_{c}^{d}$ and $\mathcal{A}_{\sigma}\left(\mathscr{E}_{1}^{d} \cap \mathcal{K}_{c}^{d}\right)=\mathcal{A}_{\sigma}\left(\mathscr{E}_{1}^{d}\right) \cap \mathcal{K}_{c}^{d}$ hold. Since (by the very definition of Borel sets) moreover obviously $\mathcal{B}\left(\left(\mathcal{K}_{c}^{d}, \delta_{H}\right)\right)=\mathcal{B}\left(\left(\mathcal{K}^{d}, \delta_{H}\right)\right) \cap \mathcal{K}_{c}^{d}$ is satisfied the proposition follows immediately from Theorem 1.20.

\subsubsection{Support functions}

Every non-empty convex and compact set $A \in \mathcal{K}_{c}^{d}$ can be described by its so-called support function $s_{A}(\cdot)$, defined by

$$
s_{A}(u):=\sup _{a \in A}\langle a, u\rangle \quad \text { for every } u \in \mathbb{R}^{d},
$$


whereby $\langle\cdot, \cdot\rangle$ denotes the standard inner product on $\mathbb{R}^{d}$.

Since the mapping $a \mapsto\langle a, u\rangle$, for fixed $u \in \mathbb{R}^{d}$, is continuous on $\mathbb{R}^{d}$ and every set $A \in \mathcal{K}_{c}^{d}$ is compact it follows immediately that the supremum in (1.11) actually is a maximum, i.e.

$$
s_{A}(u)=\max _{a \in A}\langle a, u\rangle \quad \text { for every } u \in \mathbb{R}^{d} .
$$

Due to the bilinearity of the inner product support functions frequently are only defined on the $d$-dimensional sphere $S^{d-1}:=\left\{x \in \mathbb{R}^{d}:\|x\|_{2}=1\right\}$.

Moreover considering $u \in S^{d-1}$ the intuitive meaning of support functions becomes clear: By definition $A$ is contained in the halfspace

$$
H^{-}(A, u):=\left\{x \in \mathbb{R}^{d}:\langle x, u\rangle \leq s_{A}(u)\right\}
$$

generated by the hyperplane

$$
H(A, u):=\left\{x \in \mathbb{R}^{d}:\langle x, u\rangle=s_{A}(u)\right\}
$$

Furthermore there exists at least one point $a_{u} \in A$ lying on the hyperplane $H(A, u) . H^{-}(A, u)$ and $H(A, u)$ are referred to as supporting halfspace and supporting hyperplane with outer normal $u$ to the set $A$ respectively.

Consequently for $u \in S^{d-1}$ the value $s_{A}(u)$ is the signed distance of the hyperplane $H(A, u)$ with exterior normal $u$ to the origin (compare Figure 1.1).

\section{Example 1.22}

- The support function $s_{I}$ of an interval $I=[a, b] \in \mathcal{K}_{c}^{1}$ in $\mathbb{R}$ calculates to $s_{I}(1)=b$ and $s_{I}(-1)=-a$.

- The support function $s_{K}$ of a $d$-dimensional closed ball $K=\bar{B}(0, R)$ with center 0 and radius $R$ is given by $s_{K}(u)=R$ for all $u \in S^{d-1}$.

- The support function $s_{E}$ of a $d$-dimensional ellipsoid

$$
E=\left\{x \in \mathbb{R}^{d}: \sum_{i=1}^{d}\left(\frac{x_{i}}{a_{i}}\right)^{2} \leq 1\right\}
$$

is given by $s_{E}(u)=\sqrt{\left(\sum_{i=1}^{d} u_{i}^{2} a_{i}^{2}\right)}$ for all $u \in S^{d-1}$.

Some of the most important properties of support functions of sets in $\mathcal{K}_{c}^{d}$ are summarized in the next theorem.

Since by definition the $\alpha$-cuts $\left[\xi^{\star}\right]_{\alpha}$ of a $d$-dimensional fuzzy vector $\xi^{\star} \in \mathcal{F}_{c}^{d}$ are elements in $\mathcal{K}_{c}^{d}$, these properties are also fulfilled by support functions of fuzzy vectors, which will be demonstrated in the next section. 
Figure 1.1: Geometric meaning of support functions

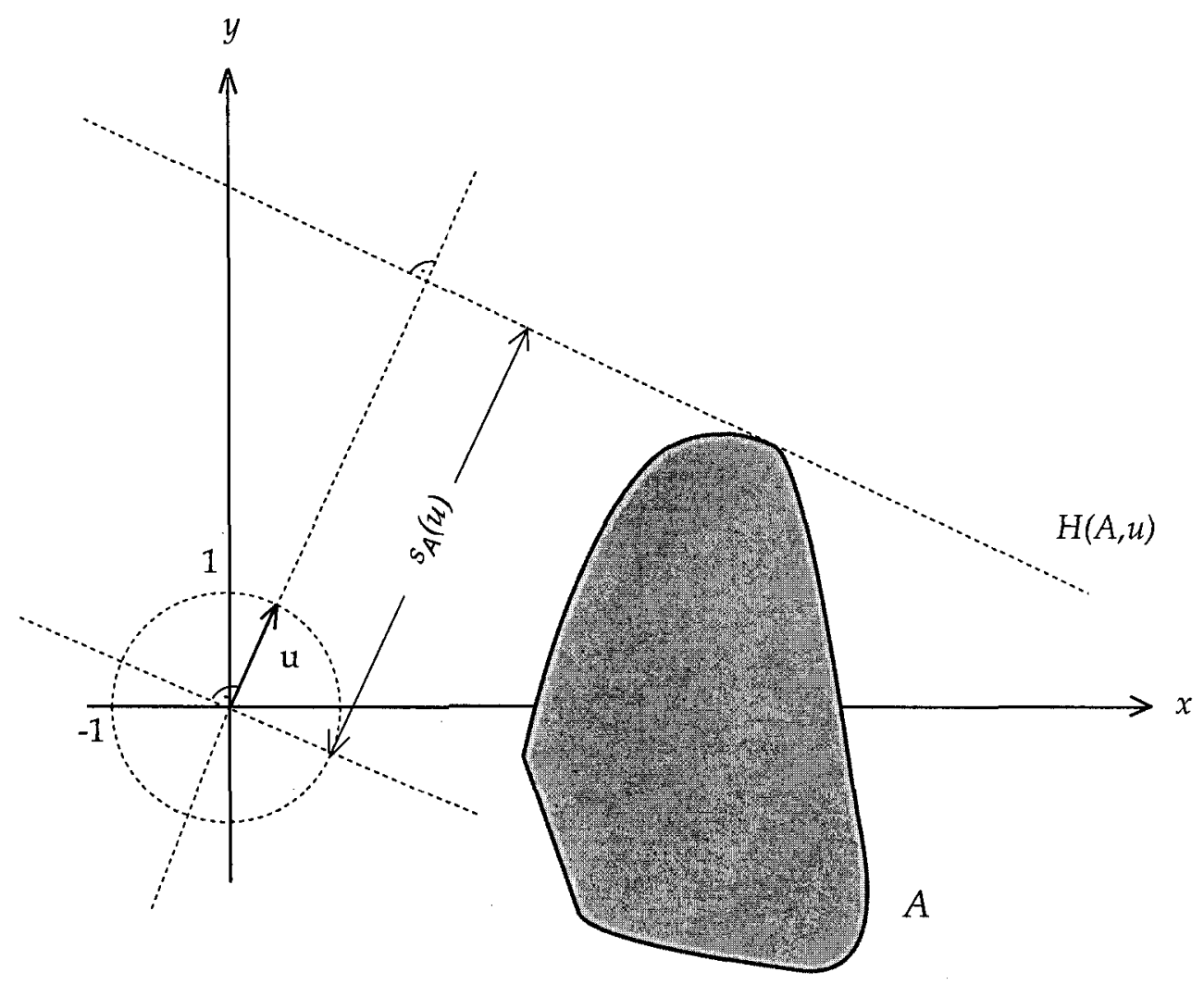

Theorem 1.23 Suppose that $K, L \in \mathcal{K}_{c}^{d}$ and denote by $s_{K}(\cdot)$ and $s_{L}(\cdot)$ the corresponding support functions defined according to (1.11), then the following assertions hold:

1. $s_{K}(\cdot)$ is positive homogeneous and subadditive, i.e. $s_{K}(\lambda u)=\lambda s_{K}(u)$ and $s_{K}(u+v) \leq s_{K}(u)+s_{K}(v)$ for every $u, v \in \mathbb{R}^{d}$ and $\lambda \geq 0$.

2. $s_{K}(\cdot)$ is Lipschitz continuous on $\mathbb{R}^{d}$.

3. If $K, L \subseteq \bar{B}(0, R)$, where $\bar{B}(0, R)$ denotes the closed ball with radius $R$ and center 0 , then

$$
\left|s_{K}(u)-s_{L}(v)\right| \leq R\|u-v\|_{2}+\delta_{H}(K, L) \max \left\{\|u\|_{2},\|v\|_{2}\right\} .
$$

Proof: In order to prove the theorem let $K, L \in \mathcal{K}_{c}^{d}, u, v \in \mathbb{R}^{d}$ and $\lambda \geq 0$. Using the bilinearity of the inner product it follows that

$$
s_{K}(u+v)=\max _{a \in K}\langle a, u+v\rangle \leq \max _{a \in K}\langle a, u\rangle+\max _{a \in K}\langle a, v\rangle=s_{K}(u)+s_{K}(v),
$$




$$
s_{K}(\lambda u)=\max _{a \in K}\langle a, \lambda u\rangle=\lambda \max _{a \in K}\langle a, u\rangle=\lambda s_{K}(u)
$$

which proves the first assertion.

Since $K, L \in \mathcal{K}_{c}^{d}$ are compact by definition, there exists a positive real number $R$ such that $K \cup L \subseteq \bar{B}(0, R)$.

On the one hand given $K \in \mathcal{K}_{c}^{d}$ and $u \in \mathbb{R}^{d}$ there exists $a_{0} \in K$ such that $s_{K}(u)=\left\langle a_{0}, u\right\rangle$. Furthermore by the definition of the Hausdorff metric $\delta_{H}$ there exists $b_{0} \in L$ fulfilling $\left\|a_{0}-b_{0}\right\|_{2} \leq \delta_{H}(K, L)$. Consequently using the Cauchy Schwarz inequality it follows that

$$
\begin{aligned}
s_{K}(u)-s_{L}(v) & \leq\left\langle a_{0}, u\right\rangle-\left\langle b_{0}, v\right\rangle \leq\left|\left\langle a_{0}, u\right\rangle-\left\langle a_{0}, v\right\rangle\right|+\left|\left\langle a_{0}, v\right\rangle-\left\langle b_{0}, v\right\rangle\right| \\
& \leq\left\|a_{0}\right\|_{2}\|u-v\|_{2}+\delta_{H}(K, L)\|v\|_{2} \\
& \leq R\|u-v\|_{2}+\delta_{H}(K, L)\|v\|_{2} .
\end{aligned}
$$

On the other hand starting with $L \in \mathcal{K}_{c}^{d}$ and $v \in \mathbb{R}^{d}$ using completely the same argument yields

$$
s_{L}(v)-s_{K}(u) \leq R\|u-v\|_{2}+\delta_{H}(K, L)\|u\|_{2} .
$$

From this, inequality (1.12) follows immediately.

For $K=L$ inequality (1.12) reduces to $\left|s_{K}(u)-s_{K}(v)\right| \leq R\|u-v\|_{2}$, which shows that $s_{K}(\cdot)$ is Lipschitz continuous and completes the proof.

The above Theorem 1.23 provides the possibility of embedding $\mathcal{K}_{c}^{d}$ isometrically and structure-preserving in the Banach space $\left(C\left(S^{d-1}\right),\|\cdot\|_{\infty}\right)$ of all real-valued continuous functions on the $d$-dimensional unit sphere with the maximum norm $\|\cdot\|_{\infty}$ :

Theorem 1.24 Define a mapping $\Phi: \mathcal{K}_{c}^{d} \rightarrow C\left(S^{d-1}\right)$ by $\Phi(K):=s_{K}$, then

- $\Phi$ is isometric, i.e. $\|\Phi(K)-\Phi(L)\|_{\infty}=\left\|s_{K}-s_{L}\right\|_{\infty}=\delta_{H}(K, L)$ for all $K, L \in \mathcal{K}_{c}^{d}$.

- $\Phi$ preserves structure, i.e. $\Phi(K+L)=s_{K+L}=s_{K}+s_{L}=\Phi(K)+\Phi(L)$ and $\Phi(\lambda K)=s_{\lambda K}=\lambda s_{K}=\lambda \Phi(K)$ for all $K, L \in \mathcal{K}_{c}^{d}$ and $\lambda \geq 0$.

- $\Phi$ preserves order, i.e. $K \subseteq L \Leftrightarrow s_{K}(u) \leq s_{L}(u) \forall u \in S^{d-1}$.

Proof: First of all notice that the assertion that $\Phi$ preserves structure immediately follows from the fact that $s_{K}(u)=\max _{a \in A}\langle a, u\rangle$ and the definition of the Minkowski sum and Minkowski scalar multiplication.

Furthermore the third point is an obvious consequence of Theorem 1.27, which says that every set $K \in \mathcal{K}_{c}^{d}$ can be reconstructed from its support 
function $s_{K}(\cdot)$. Consequently only the first assertion remains to be proved. Applying inequality (1.12) for $u=v \in S^{d-1}$ yields

$$
\left|s_{K}(u)-s_{L}(u)\right| \leq \delta_{H}(K, L),
$$

which immediately shows that $\left\|s_{K}-s_{L}\right\|_{\infty} \leq \delta_{H}(K, L)$.

On the other hand assume that $\left\|s_{K}-s_{L}\right\|_{\infty}=: \delta_{0}$. Then for all $u \in S^{d-1}$ both $s_{K}(u) \leq s_{L}(u)+\delta_{0}$ and $s_{L}(u) \leq s_{K}(u)+\delta_{0}$ hold. Since obviously $\delta_{0}=s_{\bar{B}\left(0, \delta_{0}\right)}(u)$ holds for every $u \in S^{d-1}$ this shows that

$$
\begin{aligned}
& s_{K}(u) \leq s_{L}(u)+s_{\bar{B}\left(0, \delta_{0}\right)}(u)=s_{L+\bar{B}\left(0, \delta_{0}\right)}(u) \quad \text { and } \\
& s_{L}(u) \leq s_{K}(u)+s_{\bar{B}\left(0, \delta_{0}\right)}(u)=s_{K+\bar{B}\left(0, \delta_{0}\right)}(u) \quad \forall u \in S^{d-1} .
\end{aligned}
$$

Applying Theorem 1.27 these inequalities imply that $K \subseteq L+\bar{B}\left(0, \delta_{0}\right)$ as well as $L \subseteq K+\bar{B}\left(0, \delta_{0}\right)$, which, using Lemma 1.10 , proves that $\delta_{H}(K, L) \leq \delta_{0}$. This completes the proof of the theorem.

Based on Theorem 1.24 further properties of the Hausdorff metric $\delta_{H}$ on $\mathcal{K}_{c}^{d}$ concerning the Minkowski operations can easily be proved:

Proposition 1.25 For non-empty convex and compact sets $K, L, T \in \mathcal{K}_{c}^{d}$, and $c \in \mathbb{R}$ the following assertions hold:

- $\delta_{H}(K+T, L+T)=\delta_{H}(K, L) \quad$ (translation invariance)

- $\delta_{H}(c K, c L)=|c| \delta_{H}(K, L)$

Proof: Because of the fact that $\Phi$ is isometric it follows that

$$
\begin{aligned}
\delta_{H}(K+T, L+T) & =\max _{u \in S^{d-1}}\left|s_{K+T}(u)-s_{L+T}(u)\right| \\
& =\max _{u \in S^{d-1}}\left|s_{K}(u)+s_{T}(u)-s_{L}(u)-s_{T}(u)\right| \\
& =\max _{u \in S^{d-1}}\left|s_{K}(u)-s_{L}(u)\right|=\delta_{H}(K, L)
\end{aligned}
$$

The second point can be proved in completely the same manner.

In order to continue with properties of support functions the following simple lemma that describes the (strong) separation of a closed convex set $A$ and a point $x$ lying outside that set by means of a separating hyperplane is helpful (compare [37] for more results on separation of convex sets).

Lemma 1.26 Suppose that $A \subseteq \mathbb{R}^{d}$ is closed and convex and that $x \notin A$, then there exists $u \in S^{d-1}$ and $\alpha \in \mathbb{R}$ such that

$$
\langle y, u\rangle \leq \alpha<\langle x, u\rangle, \forall y \in A .
$$

In other words, there exists a hyperplane $H_{u, \alpha}=\left\{y \in \mathbb{R}^{d}:\langle y, u\rangle=\alpha\right\}$, such that $A \subseteq H_{u, \alpha}^{-}=\left\{y \in \mathbb{R}^{d}:\langle y, u\rangle \leq \alpha\right\}$ and $x \in\left(H_{u, \alpha}^{-}\right)^{c}$. 
Proof: First of all it is easy to show that there is a unique point $p(A, x)$ in $A$ nearest to the point $x$ :

Choose $r>0$ such that $\bar{B}(x, r) \cap A$ is non-empty and compact. Consequently the continuous function $y \mapsto\|x-y\|_{2}$ attains a minimum at $y_{0}$ on $\bar{B}(x, r) \cap A$. This minimizing point is unique since if there was $y_{1} \in$ $\bar{B}(x, r) \cap A$ with $y_{1} \neq y_{0}$ fulfilling $\left\|x-y_{0}\right\|_{2}=\left\|x-y_{1}\right\|_{2}$, then choosing $z:=\frac{1}{2}\left(y_{0}+y_{1}\right) \in \bar{B}(x, r) \cap A$ would give $\|x-z\|_{2}<\left\|x-y_{0}\right\|_{2}$, which is a contradiction to the choice of $y_{0}$.

Having that define a vector $u=u(A, x) \in S^{d-1}$ by

$$
u=u(A, x):=\frac{x-p(A, x)}{\|x-p(A, x)\|_{2}}
$$

and set $\alpha:=\langle p(A, x), u\rangle$.

Suppose there exists a point $w \in A$ with $w \notin H_{u, \alpha}^{-}=\left\{y \in \mathbb{R}^{d}:\langle y, u\rangle \leq \alpha\right\}$ then by the first part of the proof there exists a point $z$ in the line segment $[p(A, x), w]$ from $p(A, x)$ to $w$ nearest to $x$. Since $A$ is convex, it follows that $z \in A$ and $\|x-z\|_{2}<\|x-p(A, x)\|_{2}$, which contradicts the construction of $p(A, x)$. Consequently $A \subseteq H_{u, \alpha}^{-}$holds.

Due to $x \notin A$ obviously $\langle x-p(A, x), x-p(A, x)\rangle=\|x-p(A, x)\|_{2}^{2}>0$ holds, which is equivalent to $\langle x, u\rangle\rangle\langle p(A, x), u\rangle=\alpha$ and therefore completes the proof of the theorem.

Theorem 1.27 Suppose that $s_{A}(\cdot)$ is the support function of a given set $A \in \mathcal{K}_{c}^{d}$, then $A$ can be reconstructed by

$$
A=\left\{a \in \mathbb{R}^{d}:\langle a, u\rangle \leq s_{A}(u) \forall u \in S^{d-1}\right\} .
$$

Proof: Set $\tilde{A}=\left\{a \in \mathbb{R}^{d}:\langle a, u\rangle \leq s_{A}(u) \forall u \in S^{d-1}\right\}$.

Then obviously $A \subseteq \tilde{A}$ follows directly from the the definition of $s_{A}(\cdot)$.

In order to prove the other inclusion assume that $x \notin A$. Using Lemma 1.26 it follows that there exist $u \in S^{d-1}$ and $\alpha \in \mathbb{R}$ such that

$$
\langle y, u\rangle \leq \alpha<\langle x, u\rangle \quad \forall y \in A .
$$

This shows that $s_{A}(u) \leq \alpha<\langle x, u\rangle$ and therefore $x \notin \tilde{A}$ holds, proving that $\tilde{A} \subseteq A$.

The next theorem describes which conditions a function $f: \mathbb{R}^{d} \rightarrow \mathbb{R}$ has to fulfill in order to be a support function of a set $K \in \mathcal{K}_{c}^{d}$.

Theorem 1.28 If a function $f: \mathbb{R}^{d} \rightarrow \mathbb{R}$ is subadditive and positive homogeneous then there exists a unique set $K \in \mathcal{K}_{c}^{d}$ such that $f$ is the support function of $K$, i.e. $f=s_{K}$. 
Proof: Obviously $f$ is bounded on the finite set $\left\{e_{1},-e_{1}, e_{2},-e_{2} \cdots, e_{d},-e_{d}\right\}$, where $\left\{e_{1}, e_{2}, \cdots, e_{d}\right\}$ denotes the canonical basis of the vector space $\mathbb{R}^{d}$.

Consequently by using subadditivity and positive homogenity it follows that $f$ is bounded on $S^{d-1}$ by a constant $R$, which in turn implies that for every $u \in \mathbb{R}^{d} \backslash\{0\}$

$$
|f(u)|=\left|f\left(\frac{\|u\|_{2} u}{\|u\|_{2}}\right)\right|=\|u\|_{2}\left|f\left(\frac{u}{\|u\|_{2}}\right)\right| \leq R\|u\|_{2}
$$

holds. Furthermore $f(0)=0$ immediately follows from positive homogenity. Given arbitrary $u, v \in \mathbb{R}^{d}$ and again using subadditivity it therefore follows that

$$
\begin{aligned}
& f(u)-f(v) \leq f(u-v) \leq|f(u-v)| \leq R\|u-v\|_{2} \\
& f(v)-f(u) \leq f(v-u) \leq|f(v-u)| \leq R\|u-v\|_{2},
\end{aligned}
$$

which gives $|f(u)-f(v)| \leq R\|u-v\|_{2}$, and proves that $f$ is Lipschitz continuous on $\mathbb{R}^{d}$. Define a set $K$ by

$$
K:=\left\{a \in \mathbb{R}^{d}:\langle a, u\rangle \leq f(v) \forall u \in S^{d-1}\right\}
$$

Then it follows immediately that $K$ is as intersection of closed halfspaces itself closed and that $K$ is bounded because $f$ is bounded on $S^{d-1}$. Since the convexity of $K$ is a trivial consequence of the linearity of the inner product it follows that $K$ is convex and compact.

Furthermore obviously $s_{K}(u) \leq f(u)$ holds for every $u \in S^{d-1}$ and therefore for every $u \in \mathbb{R}^{d}$ by positive homogenity.

It remains to prove that $K \neq \emptyset$ and that $f(u) \leq s_{K}(u)$ for all $u \in S^{d-1}$.

Define the so-called epigraph $\Gamma^{+} \subseteq \mathbb{R}^{d+1}$ of the function $f$ by

$$
\Gamma^{+}:=\left\{(v, t) \in \mathbb{R}^{d} \times \mathbb{R}: f(v) \leq t\right\} .
$$

It follows immediately that $\Gamma^{+}$is a closed convex cone, i.e. it is closed, convex and for every $z \in \Gamma^{+}$and $\lambda \geq 0 \lambda z \in \Gamma^{+}$holds. Since for every $u \in \mathbb{R}^{d}$ $(u, f(u))$ is a boundary point of $\Gamma^{+}$Theorem 1.3.2. and Theorem 1.3.9 in [37] show that there exists $(y, \eta) \in \mathbb{R}^{d} \times \mathbb{R}$ with $(y, \eta) \neq 0 \in \mathbb{R}^{d+1}$ determining a (supporting) hyperplane $H=\left\{(z, t) \in \mathbb{R}^{d} \times \mathbb{R}:\langle(z, t),(y, \eta)\rangle=0\right\}$ going through $(u, f(u))$ and the origin $\mathbf{0}$ and fulfilling

$$
\Gamma^{+} \subseteq H=\left\{(z, t) \in \mathbb{R}^{d} \times \mathbb{R}:\langle(z, t),(y, \eta)\rangle \leq 0\right\} .
$$

Since $\eta=0$ implies $\langle y, v\rangle \leq 0$ for all $v \in \mathbb{R}^{d}$, giving $y=0$ and contradicting the assumption $(y, \eta) \neq 0$, it follows that $\eta \neq 0$.

Furthermore if $\eta>0$ then for every $v \in \mathbb{R}^{d}$

$$
\langle(y, \eta),(v, t)\rangle=\langle y, v\rangle+t \eta \leq 0
$$


would have to hold for every $t \geq f(v)$. This shows that $\eta<0$.

Set $\tilde{y}:=\frac{y}{|\eta|}$ then (1.14) can be written as

$$
\Gamma^{+} \subseteq\left\{(z, t) \in \mathbb{R}^{d} \times \mathbb{R}:\langle z, \tilde{y}\rangle \leq t\right\} .
$$

For every $v \in \mathbb{R}^{d}(v, f(v))$ lies in $\Gamma^{+}$by definition, so $\langle v, \tilde{y}\rangle \leq f(v)$ holds for every $v \in \mathbb{R}^{d}$ which proves that $\tilde{y} \in K \neq \emptyset$.

Finally using $s_{K}(u) \geq\langle\tilde{y}, u\rangle=f(u)$ completes the proof of the theorem since $u \in \mathbb{R}^{d}$ was arbitrary.

With the aid of Theorem 1.28 it can be shown that pointwise convergence of a sequence $\left(s_{K_{n}}\right)_{n \in \mathbb{N}}$ of support functions on $S^{d-1}$ implies uniform convergence to a support function $s_{K}$ on $S^{d-1}$.

Theorem 1.29 Suppose that $\left(K_{n}\right)_{n \in \mathbb{N}}$ is a sequence in $\mathcal{K}_{c}^{d}$ such that the corresponding support functions $\left(s_{K_{n}}\right)_{n \in \mathbb{N}}$ converge pointwise on $S^{d-1}$.

Then there exists a unique set $K \in \mathcal{K}_{c}^{d}$ such that $s_{K_{n}}$ converges to $s_{K}$ uniformly on $S^{d-1}$.

Proof: Set $s_{n}:=s_{K_{n}}$ for every $n \in \mathbb{N}$. Since $s_{n}$ converges pointwise on $S^{d-1}$ it follows from positive homogenity that it converges on whole $\mathbb{R}^{d}$. Furthermore because of the obvious fact that the limit function $s$ inherits positive homogenity and subbadditivity according to Theorem $1.28 \mathrm{~s}(\cdot)$ is the support function of a unique set $K \in \mathcal{K}_{c}^{d}$, i.e. $s=s_{K}$.

For every $u \in S^{d-1}$ set $\alpha(u):=\sup \left\{s_{n}(u): n \in \mathbb{N}\right\}$, then it follows from pointwise convergence that $\alpha(u)<\infty$ for every $u \in S^{d-1}$.

Again by considering the finite set $\mathfrak{E}=\left\{e_{1},-e_{1}, e_{2},-e_{2} \cdots, e_{d},-e_{d}\right\}$, where $\left\{e_{1}, e_{2}, \cdots, e_{d}\right\}$ denotes the canonical basis of the vector space $\mathbb{R}^{d}$, it follows that $R=\max \{\alpha(u): u \in \mathfrak{E}\}<\infty$. Hence for $L:=\sqrt{d} R$ both

$$
K_{n} \subseteq \bar{B}(0, L) \text { and } K \subseteq \bar{B}(0, L)
$$

hold, which according to inequality (1.12) implies that $s, s_{1}, s_{2}, \ldots$ are Lipschitz continuous with common Lipschitz constant $L$ and therefore are a equicontinuous family.

Having that the proof is easily completed by using the compactness of $S^{d-1}$ in the following way:

For arbitrary $\epsilon>0$ there exists an $\frac{\epsilon}{3 L}$-net $\mathcal{N}=\left\{x_{1}, \cdots, x_{k}\right\} \subseteq S^{d-1}$ for the sphere $S^{d-1}$. Since $\mathcal{N}$ is finite the convergence of $\left(s_{n}\right)_{n \in \mathbb{N}}$ on $\mathcal{N}$ is uniform and there exists an index $n_{0}=n_{0}(\epsilon) \in \mathbb{N}$ such that

$$
\left|s_{n}(v)-s(v)\right| \leq \frac{\epsilon}{3} \quad \forall v \in \mathcal{N}, \forall n \geq n_{0} .
$$


Given arbitrary $u \in S^{d-1}$ there exists $v_{u} \in \mathcal{N}$ such that $\left\|u-v_{u}\right\|_{2} \leq \frac{\epsilon}{3 L}$, which finally implies that

$$
\begin{aligned}
\left|s_{n}(u)-s(u)\right| & \leq\left|s_{n}(u)-s\left(v_{u}\right)+\right| s_{n}\left(v_{u}\right)-s\left(v_{u}\right)|+| s\left(v_{u}\right)-s(u) \mid \leq \\
& \leq L \frac{\epsilon}{3 L}+\frac{\epsilon}{3}+L \frac{\epsilon}{3 L}=\epsilon \quad \forall n \geq n_{0} .
\end{aligned}
$$

This proves uniform convergence since $u \in S^{d-1}$ was arbitrary.

The required pointwise convergence condition can even be alleviated to almost everywhere convergence on $S^{d-1}$ with respect to the normalized Lebesgue (surface) measure $\vartheta$ on the unit sphere $S^{d-1}$ :

Theorem 1.30 Suppose that $\left(K_{n}\right)_{n \in \mathbb{N}}$ is a sequence in $\mathcal{K}_{c}^{d}$ such that the corresponding support functions $\left(s_{K_{n}}\right)_{n \in \mathbb{N}}$ converge $\vartheta$-almost everywhere on $S^{d-1}$. Then there exists a unique set $K \in \mathcal{K}_{c}^{d}$ such that $s_{K_{n}}$ converges to $s_{K}$ uniformly on $S^{d-1}$.

Proof: Again set $s_{n}(u):=s_{K_{n}}(u)$ for every $n \in \mathbb{N}$ and every $u \in S^{d-1}$.

If $s_{n}(u)$ converges for $\vartheta$-almost every $u \in S^{d-1}$, then it follows that $\alpha(u):=$ $\sup \left\{s_{n}(u): n \in \mathbb{N}\right\}$ is finite for $\vartheta$-almost every $u \in S^{d-1}$.

Since every relative open set $V \subseteq S^{d-1}$ has positive mass $\vartheta(V)>0$ it follows that every measurable set $A \subseteq S^{d-1}$ with $\vartheta(A)=1$ is dense in $S^{d-1}$. Hence $\alpha(u)<\infty$ on a dense subset $A$ of $S^{d-1}$, which shows that $M$ defined by

$$
M:=\bigcap_{u \in S^{d-1}}\left\{x \in \mathbb{R}^{d}:\langle x, u\rangle \leq \alpha(u)\right\}
$$

is bounded. Since $M$ is as intersection of closed sets itself closed this implies that $M$ is compact. Consequently there exists a constant $L \in \mathbb{R}^{+}$such that $M$ is contained in the closed ball $\bar{B}(0, L)$. Therefore

$$
K_{n} \subseteq M \subseteq \bar{B}(0, L)
$$

holds for every $n \in \mathbb{N}$, which according to (1.12) implies that all functions $s_{K_{1}}, s_{K_{2}}, \ldots$ are Lipschitz continuous with common Lipschitz constant $L$.

Suppose now that $v \in S^{d-1}$ and let $\epsilon>0$ be arbitrary but fixed, then there exists $u \in A \subseteq S^{d-1}$ such that $\|u-v\|_{2}<\frac{\epsilon}{3 L}$ and an integer $n_{0}=n_{0}(\epsilon, u) \in \mathbb{N}$ such that

Consequently

$$
\left|s_{n}(u)-s_{m}(u)\right|<\frac{\epsilon}{3} \quad \forall n, m \geq n_{0} .
$$

$$
\begin{aligned}
\left|s_{n}(v)-s_{m}(v)\right| & \leq\left|s_{n}(v)-s_{n}(u)\right|+\left|s_{n}(u)-s_{m}(u)\right|+\left|s_{m}(u)-s_{m}(v)\right| \leq \\
& \leq L \frac{\varepsilon}{3 L}+\frac{\epsilon}{3}+L \frac{\varepsilon}{3 L} \leq \epsilon \quad \forall n, m \geq n_{0}
\end{aligned}
$$

holds, which proves that $\left(s_{n}\right)_{n \in \mathbb{N}}$ converges pointwise everywhere on $S^{d-1}$ since $v \in S^{d-1}$ was arbitrary. Applying Theorem 1.29 completes the proof. 


\subsection{3 $\quad L^{p}$-metrics induced by support functions}

The one-to-one correspondence of non-empty, convex and compact sets $K \in \mathcal{K}_{c}^{d}$ and their support functions $s_{K}$ as described before makes it possible to define metrics $\rho_{p}$ on $\mathcal{K}_{c}^{d}, p \in[1, \infty)$, via the $L^{p}$-norms of the corresponding support functions on $S^{d-1}$ with respect to the normalized Lebesgue measure $\vartheta$ :

$$
\rho_{p}(K, L):=\left\|s_{K}-s_{L}\right\|_{p}=\left(\int_{S^{d-1}}\left|s_{K}(u)-s_{L}(u)\right|^{p} d \vartheta(u)\right)^{\frac{1}{p}}
$$

According to Theorem 1.23 for every set $K \in \mathcal{K}_{c}^{d}$ the corresponding support function $s_{K}$ is an element in the Banach space $\left(C\left(S^{d-1}\right),\|\cdot\|_{\infty}\right)$, which ensures that the integral in (1.15) exists for every $p \in[1, \infty)$.

By using the well known properties of the $p$-norm $\|\cdot\|_{p}$ it is easy to see that $\rho_{p}(\cdot, \cdot)$ is non-negative, commutative and fulfills the triangle inequality. Furthermore if $\rho_{p}(K, L)=0$ then it follows immediately that $s_{K}(u)=s_{L}(u)$ $\vartheta$-almost everywhere, which, using continuity, implies that $s_{K}(u)=s_{L}(u)$ holds for every $u \in S^{d-1}$. Therefore $K=L$ holds, which completes the proof that $\rho_{p}$ is a metric on $\mathcal{K}_{c}^{d}$ for every $p \in[1, \infty)$.

Analyzing the interrelation of the metric $\rho_{p}$ and the Hausdorff metric $\delta_{H}$ Theorem 1.24 (point one) immediately shows that

$$
\rho_{p}(K, L) \leq \delta_{H}(K, L)
$$

holds for every $p \in[1, \infty)$ and $K, L \in \mathcal{K}_{c}^{d}$.

Concerning an estimation in the other direction only the case $d=1$ is obvious: If $K=[a, b]$ and $L=[c, d]$ are compact intervals then it follows that $\rho_{p}(K, L)=\left(\frac{1}{2}|d-b|^{p}+\frac{1}{2}|c-a|^{p}\right)^{1 / p}$. Since $\delta_{H}(K, L)=\max \{|d-b|,|c-a|\}$ this implies that $\delta_{H}(K, L) \leq 2 \rho_{p}(K, L)$ for every $p \in[1, \infty)$, which together with (1.16) proves that $\rho_{p}$ and $\delta_{H}$ are equivalent metrics for $d=1$.

In 1984 Vitale [43] proved amongst other things the following useful inequality for $d \geq 2, p \in[1, \infty)$ and $K, L \in \mathcal{K}_{c}^{d}$ :

$$
\left(\frac{B(p+1, d-1)}{B\left(\frac{1}{2}, \frac{d-1}{2}\right) D^{d-1}}\right)^{\frac{1}{p}}\left(\delta_{H}(K, L)\right)^{\frac{p+d-1}{p}} \leq \rho_{p}(K, L)
$$

Thereby $0<D=\max \left\{\|a-b\|_{2}: a, b \in K \cup L\right\}=: \operatorname{diam}(K \cup L)$ is the diameter of $K \cup L$ and $B(\cdot, \cdot)$ denotes the beta-function. (In the trivial case of $\operatorname{diam}(K \cup L)=0$ obviously $\delta_{H}(K, L)=\rho_{p}(K, L)=0$ holds.)

This inequality is the main tool for proving the following theorem (compare Vitale [43]): 
Theorem 1.31 For every $p \in[1, \infty)$ the metric $\rho_{p}$ defined according to (1.15) induces the same topology on $\mathcal{K}_{c}^{d}$ as the Hausdorff metric $\delta_{H}$.

Moreover for every $p \in[1, \infty)\left(\mathcal{K}_{c}^{d}, \rho_{p}\right)$ is a complete, separable metric space in which every closed, bounded subset is compact.

Proof: For the case $d=1$ it is an immediate consequence of the equivalence of the metrics $\delta_{H}$ and $\rho_{p}$ that $\delta_{H}\left(K_{n}, K\right) \rightarrow 0$ for $n \rightarrow \infty$ holds exactly if $\rho_{p}\left(K_{n}, K\right) \rightarrow 0$ for $n \rightarrow \infty$ holds.

If $d \geq 2$ then (1.16) shows that $\delta_{H}\left(K_{n}, K\right) \rightarrow 0$ for $n \rightarrow \infty$ implies that $\rho_{p}\left(K_{n}, K\right) \rightarrow 0$ for $n \rightarrow \infty$.

In order to prove the opposite implication assume that $\rho_{p}\left(K_{n}, K\right) \rightarrow 0$ for $n \rightarrow \infty$. Since it follows directly from the definition of the Hausdorff metric $\delta_{H}$ that for every $K, L \in \mathcal{K}_{c}^{d}$

$$
\operatorname{diam}(K \cup L) \leq \operatorname{diam}(K)+2 \delta_{H}(K, L)
$$

holds, setting $C(d, p):=\left[\frac{B(p+1, d-1)}{B\left(\frac{1}{2}, \frac{d-1}{2}\right)}\right]^{1 / p}$ inequality (1.17) applied for $K_{n}, K$ shows that

$$
C(d, p) \frac{\left(\delta_{H}\left(K_{n}, K\right)\right)^{1+\frac{d-1}{p}}}{\left(\operatorname{diam}(K)+2 \delta_{H}\left(K_{n}, K\right)\right)^{\frac{d-1}{p}}} \leq \rho_{p}\left(K_{n}, K\right)
$$

- If $\operatorname{diam}(K)=0$ then (1.18) reduces to $\frac{C(d, p)}{2} \delta_{H}\left(K_{n}, K\right) \leq \rho_{p}\left(K_{n}, K\right)$, proving that $\delta_{H}\left(K_{n}, K\right) \rightarrow 0$ since $C(d, p) \neq 0$.

- If $\operatorname{diam}(K)>0$ then $\operatorname{diam}(K)+2 \delta_{H}\left(K_{n}, K\right) \geq \operatorname{diam}(K)>0$ for all $n \in \mathbb{N}$. Hence $\rho_{p}\left(K_{n}, K\right) \rightarrow 0$ implies $\delta_{H}\left(K_{n}, K\right) \rightarrow 0$ for $n \rightarrow \infty$.

Altogether it follows that

$$
\rho_{p}\left(K_{n}, K\right) \rightarrow 0 \Leftrightarrow \delta_{H}\left(K_{n}, K\right) \rightarrow 0 \text { for } n \rightarrow \infty .
$$

Consequently for every $d \geq 1$ a set $\mathcal{C} \subseteq \mathcal{K}_{c}^{d}$ is closed with respect to $\delta_{H}$ if and only if it is closed with respect to $\rho_{p}$, which proves that the metrics $\rho_{p}$ and $\delta_{H}$ induce the same topology. This completes the proof of the first part of the theorem.

For the case $d=1$ obviously a set $\mathcal{C} \subseteq \mathcal{K}_{c}^{1}$ is bounded with respect to $\delta_{H}$ exactly if it is bounded with respect to $\rho_{p}$ since the metrics are equivalent. Suppose now that $d \geq 2$ and that $\mathcal{C} \subseteq \mathcal{K}_{c}^{d}$ is bounded with respect to the metric $\rho_{p}$. Then there exists a set $M \in \mathcal{K}_{c}^{d}$ and $R>0$ such that $\mathcal{C} \subseteq \bar{B}_{\rho_{p}}(M, R)$, where $\bar{B}_{\rho_{p}}(M, R)$ denotes the closed ball with center $M$ 
and radius $R$ with respect to the metric $\rho_{p}$. By using the triangle inequality one may assume without loss of generality that $M=\{m\} \in \mathcal{K}_{c}^{d}$, which implies that $\operatorname{diam}(M)=0$. If $A \in \mathcal{C}$ then applying inequality (1.18) for $K=\{m\}$ and $K_{n}=A$ yields

$$
\frac{C(d, p)}{2} \delta_{H}(\{m\}, A) \leq \rho_{p}(\{m\}, A) \leq R .
$$

Since $C(d, p) \neq 0$ it follows that $\delta_{H}(\{m\}, A) \leq \frac{2 R}{C(d, p)}$, which shows that $\mathcal{A}$ is bounded with respect to the metric $\delta_{H}$ too.

Consequently by (1.16) for arbitrary $d \geq 1$ a set $\mathcal{C} \subseteq \mathcal{K}_{c}^{d}$ is bounded with respect to $\delta_{H}$ if and only if it is bounded with respect to $\rho_{p}$. Hence the first part of the proof implies that a set $\mathcal{C} \subseteq \mathcal{K}_{c}^{d}$ is closed and bounded with respect to $\delta_{H}$ if and only if it is closed and bounded with respect to $\rho_{p}$. Moreover the first part of the proof shows that a set $\mathcal{C} \subseteq \mathcal{K}_{c}^{d}$ is compact with respect to $\delta_{H}$ exactly if it is compact with respect to $\rho_{p}$.

Having that simply applying Theorem 1.14 shows that every closed, bounded set $\mathcal{C}$ in $\mathcal{K}_{c}^{d}$ is compact with respect to $\rho_{p}$.

Especially for every $n \in \mathbb{N}$ the closed ball $\bar{B}_{\rho_{p}}(0, n)$ is compact, which shows that $\left(\mathcal{K}_{c}^{d}, \rho_{p}\right)$ is a $\sigma$-compact metric space and therefore separable.

Because of the fact that for every Cauchy sequence $\left(K_{n}\right)_{n \in \mathbb{N}}$ the closed set $L:=\overline{\left\{K_{1}, K_{2}, K_{3}, \cdots\right\}}$ (closure with respect to $\rho_{p}$ ) is bounded, it follows that $L$ is compact and therefore that there exists a convergent subsequence $\left(K_{n_{j}}\right)_{j \in \mathbb{N}}$ and a set $K \in \mathcal{K}_{c}^{d}$ such that $\rho_{p}\left(K_{n_{j}}, K\right) \rightarrow 0$ for $j \rightarrow \infty$.

By the Cauchy property it follows immediately that $\rho_{p}\left(K_{n}, K\right) \rightarrow 0$ holds for $n \rightarrow \infty$, which finally shows that $\left(\mathcal{K}_{c}^{d}, \rho_{p}\right)$ is a complete metric space.

\subsection{Operations on $\mathcal{F}_{c}^{d}$}

As fuzzy numbers and fuzzy vectors can be seen as a generalization of real numbers and real vectors it is natural to try to define operations such as the sum of two fuzzy vectors or the scalar multiplication of a real number with a fuzzy vector so that they are natural extensions of the corresponding operations for real numbers and real vectors.

If for instance $\xi^{\star} \in \mathcal{F}_{c}^{1}$ and $c \in \mathbb{R}$ then it seems natural to define the fuzzy number $\eta^{\star}=\xi^{\star}+c$ so that $\eta^{\star}(x)=\xi^{\star}(x-c)$.

Suppose now that $T: \mathbb{R}^{d} \longrightarrow \mathbb{R}^{d^{\prime}}$ is an arbitrary function and that $\xi^{\star} \in \mathcal{F}_{c}^{d}$. Define a function $\xi_{T}^{\star}: \mathbb{R}^{d^{\prime}} \longrightarrow \mathbb{R}$ for $x \in \mathbb{R}^{d^{\prime}}$ as follows:

$$
\xi_{T}^{\star}(x)=\left\{\begin{array}{ll}
0 & \text { if } T^{-1}(\{x\})=\emptyset \\
\sup \left\{\xi^{\star}(y): y \in T^{-1}(\{x\})\right\} & \text { otherwise. }
\end{array}\right\} \forall x \in \mathbb{R}^{d^{\prime}}
$$


This construction is called Zadeh's extension principle.

In general $\xi_{T}^{\star}$ need not be a fuzzy vector, but if the function $T$ is continuous there is the following simple result:

Lemma 1.32 Suppose $T: \mathbb{R}^{d} \longrightarrow \mathbb{R}^{d^{\prime}}$ is a continuous function and $\xi^{\star} \in \mathcal{F}_{c}^{d}$. Then the function $\xi_{T}^{\star}$ defined according to (1.19) has the following properties:

1. $\left[\xi_{T}^{\star}\right]_{\alpha}=\left\{x \in \mathbb{R}^{d^{\prime}}: \xi_{T}^{\star}(x) \geq \alpha\right\} \in \mathcal{K}^{d^{\prime}}$ for every $\alpha \in(0,1]$

2. $\exists x_{0} \in \mathbb{R}^{d^{\prime}}: \xi_{T}^{\star}\left(x_{0}\right)=1$

3. $T\left(\left[\xi^{\star}\right]_{\alpha}\right)=\left[\xi_{T}^{\star}\right]_{\alpha}$ for every $\alpha \in(0,1]$

4. $\xi_{T}^{\star}(x) \in[0,1]$ for every $x \in \mathbb{R}^{d^{\prime}}$

Proof: Suppose that $\alpha \in(0,1]$ and that $x \in\left[\xi_{T}^{\star}\right]_{\alpha}$. Choose $n_{0} \in \mathbb{N}$ sufficiently large so that $1 / n<\alpha$ holds for every $n \geq n_{0}$. It follows immediately from (1.19) that for every $n \geq n_{0}$ there exists $y_{n} \in\left[\xi^{\star}\right]_{\alpha-1 / n}$ fulfilling $T\left(y_{n}\right)=x$.

The compactness of the $(\alpha-1 / n)$-cuts $\left[\xi^{\star}\right]_{\alpha-1 / n}$ for every $n \geq n_{0}$ implies the existence of a convergent subsequence $\left(y_{n_{j}}\right)_{j \in \mathbb{N}}$ with limit $y$. Using Theorem 1.4 it follows that $y \in\left[\xi^{\star}\right]_{\alpha}$, which, using continuity of $T$, yields $x \in T\left(\left[\xi^{\star}\right]_{\alpha}\right)$. On the other hand if $x \in T\left(\left[\xi^{\star}\right]_{\alpha}\right)$ then there exists $y \in\left[\xi^{\star}\right]_{\alpha}$ such that $\xi^{\star}(y) \geq \alpha>0$ and $T(y)=x$ holds. This shows that $x \in\left[\xi_{T}^{\star}\right]_{\alpha}$ and completes the proof of the third assertion of the theorem.

Because of the fact that continuity preserves compactness the compactness of $\left[\xi_{T}^{\star}\right]_{\alpha}$ is an immediate consequence of the third assertion.

The other two assertions directly follow from the definition.

If in addition the function $T$ is linear (and therefore continuous) it follows immediately that the convexity of the $\alpha$-cuts is preserved as well since linearity preserves convexity.

Hence under the requirement that $T: \mathbb{R}^{d} \longrightarrow \mathbb{R}^{d^{\prime}}$ is linear it follows that $\xi_{T}^{\star}$ is a vector-characterizing function of a $d^{\prime}$-dimensional fuzzy vector, therefore $\xi_{T}^{\star} \in \mathcal{F}_{c}^{d^{\prime}}$ holds.

This can be used in order to define a scalar multiplication of a fuzzy vector $\xi^{\star} \in \mathcal{F}_{c}^{d}$ with a real number $\lambda \in \mathbb{R}$ in the following way:

Setting $T: \mathbb{R}^{d} \longrightarrow \mathbb{R}^{d}, T(x)=\lambda x$ and applying Zadeh's extension principle gives

$$
\left(\lambda \xi^{\star}\right)(x)=\xi_{T}^{\star}(x)=\left\{\begin{array}{ll}
\mathbf{1}_{\{0\}}(x) & \text { if } \lambda=0 \\
\xi^{\star}\left(\frac{x}{\lambda}\right) & \text { otherwise }
\end{array}\right\} \forall x \in \mathbb{R}^{d} .
$$

According to the above remarks it follows that $\lambda \xi^{\star} \in \mathcal{F}_{c}^{d}$. 
Two fuzzy vectors $\xi^{\star}, \eta^{\star} \in \mathcal{F}_{c}^{d}$ can be combined to a $2 d$-dimensional fuzzy vector $\theta^{\star}$ in various ways, the probably most important method is the socalled Minimum combination rule:

$$
\theta^{\star}(x, y):=\min \left\{\xi^{\star}(x), \eta^{\star}(y)\right\} \quad \text { for all } x, y \in \mathbb{R}^{d}
$$

It is easy to see that $\theta^{\star} \in \mathcal{F}_{c}^{2 d}$ and that $\left[\theta^{\star}\right]_{\alpha}=\left[\xi^{\star}\right]_{\alpha} \times\left[\eta^{\star}\right]_{\alpha}$ for all $\alpha \in(0,1]$.

Having this the sum $\xi^{\star} \oplus \eta^{\star}$ of two fuzzy vectors $\xi^{\star}, \eta^{\star} \in \mathcal{F}_{c}^{d}$ can be defined applying Zadeh's extension principle to the function $T: \mathbb{R}^{2 d} \longrightarrow \mathbb{R}^{d}$, defined by

$$
T(x, y)=x+y \text {. }
$$

Using the above notation this gives:

$$
\begin{aligned}
\left(\xi^{\star} \oplus \eta^{\star}\right)(z)=\theta_{T}^{\star}(z) & =\sup \left\{\theta^{\star}(x, y): x, y \in \mathbb{R}^{d} \text { and } x+y=z\right\} \\
& =\sup \left\{\min \left\{\xi^{\star}(x), \eta^{\star}(y)\right\}: x, y \in \mathbb{R}^{d} \text { and } x+y=z\right\} \\
& =\max \left\{\min \left\{\xi^{\star}(x), \eta^{\star}(y)\right\}: x, y \in \mathbb{R}^{d} \text { and } x+y=z\right\}
\end{aligned}
$$

Since $T(x, y)=x+y$ is linear it follows immediately that $\xi^{\star} \oplus \eta^{\star} \in \mathcal{F}_{c}^{d}$.

This definition of the sum of two fuzzy vectors $\xi^{\star}, \eta^{\star} \in \mathcal{F}_{c}^{d}$ is closely related to the Minkowski sum on $\mathcal{K}_{c}^{d}$ (compare for example [9]):

Lemma 1.33 If $\xi^{\star}, \eta^{\star} \in \mathcal{F}_{c}^{d}$ then the $\alpha$-cut of the sum $\xi^{\star} \oplus \eta^{\star}$ is the Minkowski sum of the $\alpha$-cuts of $\xi^{\star}$ and $\eta^{\star}$, i.e.

$$
\left[\xi^{\star} \oplus \eta^{\star}\right]_{\alpha}=\left[\xi^{\star}\right]_{\alpha}+\left[\eta^{\star}\right]_{\alpha} \quad \text { for all } \alpha \in(0,1] .
$$

Proof: The assertion is a direct consequence of the fact that $\left[\theta_{T}^{\star}\right]_{\alpha}=T\left(\left[\theta^{\star}\right]_{\alpha}\right)$ with $T(x, y)=x+y$ and $\theta^{\star}(x, y)=\min \left\{\xi^{\star}(x), \eta^{\star}(y)\right\}$

The difference $\xi^{\star} \ominus \eta^{\star}$ of two fuzzy vectors $\xi^{\star}, \eta^{\star} \in \mathcal{F}_{c}^{d}$ can be defined completely in the same manner, namely applying Zadeh's extension principle to the function $T: \mathbb{R}^{2 d} \longrightarrow \mathbb{R}^{d}$ defined by

$$
T(x, y)=x-y .
$$

or, equivalently, as $\xi^{\star} \ominus \eta^{\star}:=\xi^{\star} \oplus\left(-\eta^{\star}\right)$.

Analogous to Lemma 1.33 this difference fulfills

$$
\left[\xi^{\star} \ominus \eta^{\star}\right]_{\alpha}=\left[\xi^{\star}\right]_{\alpha}-\left[\eta^{\star}\right]_{\alpha} \quad \text { for all } \alpha \in(0,1] .
$$

In order to compare two fuzzy numbers $\xi^{\star}, \eta^{\star} \in \mathcal{F}_{c}^{1}$ the following definitions 
are useful (and will be used throughout the thesis):

Starting from the semiordering $\preceq$ on the compact subintervals of $\mathbb{R}$, defined by

$$
\left[a_{1}, b_{1}\right] \preceq\left[a_{2}, b_{2}\right]: \Longleftrightarrow a_{1} \leq a_{2} \text { and } b_{1} \leq b_{2},
$$

a semiordering $\preceq$ can be defined on $\mathcal{F}_{c}^{1}$ in the following way:

$$
\xi^{\star} \preceq \eta^{\star}: \Longleftrightarrow\left[\xi^{\star}\right]_{\alpha} \preceq\left[\eta^{\star}\right]_{\alpha} \quad \forall \alpha \in(0,1]
$$

Analogous an inclusion can be defined on $\mathcal{F}_{c}^{1}$ by

$$
\xi^{\star} \subseteq \eta^{\star}: \Longleftrightarrow\left[\xi^{\star}\right]_{\alpha} \subseteq\left[\eta^{\star}\right]_{\alpha} \quad \forall \alpha \in(0,1]
$$

\subsection{Support functions for $\mathcal{F}_{c}^{d}$}

Throughout this and the next section fuzzy vectors will be denoted by capital letters $A^{\star}$ instead of greek letters $\xi^{\star}$ in order to emphasize that the main ideas come from the theory of compact and convex sets and to point out the similarities of support functions of fuzzy vectors and support functions of non-empty compact convex sets.

According to Definition 1.2 for every $\alpha \in(0,1]$ the $\alpha$-cut $\left[\xi^{\star}\right]_{\alpha}$ of every $d$-dimensional fuzzy vector $\xi^{\star} \in \mathcal{F}_{c}^{d}$ is an element of $\mathcal{K}_{c}^{d}$. Since every set $K \in \mathcal{K}_{c}^{d}$ is uniquely characterized by its support function $s_{K}$ it is natural to extend the concept of support functions to fuzzy vectors in the following way:

Suppose that $A^{\star} \in \mathcal{F}_{c}^{d}$ then the support function $s_{A^{\star}}(\cdot, \cdot)$ for $A^{\star}$ is defined by

$$
s_{A^{\star}}(u, \alpha):=s_{\left[A^{\star}\right]_{\alpha}}(u)=\max _{a \in\left[A^{\star}\right]_{\alpha}}\langle a, u\rangle,
$$

for every $u \in \mathbb{R}^{d}$ and $\alpha \in(0,1]$.

The following analogy to Theorem 1.23 holds for support functions of fuzzy vectors:

Theorem 1.34 Suppose that $A^{\star} \in \mathcal{F}_{c}^{d}$ is a d-dimensional fuzzy vector and denote by $s_{A^{*}}$ the corresponding support function defined according to (1.22), then the following assertions hold:

1. For fixed $\alpha \in(0,1] s_{A^{*}}(\cdot, \alpha)$ is positive homogeneous and subadditive.

2. For fixed $\alpha \in(0,1] s_{A^{*}}(\cdot, \alpha)$ is Lipschitz continuous.

3. For fixed $u \in \mathbb{R}^{d} s_{A^{\star}}(u, \cdot)$ is monotonically decreasing and left-continuous on $(0,1]$. 
Proof: Since $s_{A^{\star}}(u, \alpha)=s_{\left[A^{\star}\right]_{\alpha}}(u)$ holds by definition, point one and two are immediate consequences of Theorem 1.23.

Furthermore because of the fact that $\left[A^{\star}\right]_{\alpha}$ monotonically decreases as $\alpha$ increases, it is obvious that $s_{A^{\star}}(u, \cdot)$ is monotonically decreasing in $\alpha$ for fixed $u \in \mathbb{R}^{d}$. In order to prove left-continuity fix $u \in \mathbb{R}^{d}$ and assume that $\left(\alpha_{n}\right)_{n \in \mathbb{N}}$ is a sequence in $(0,1]$ that increases monotonically to the limit $\alpha \in(0,1]$. Since the sequence $\left(s_{A^{\star}}\left(u, \alpha_{n}\right)\right)_{n \in \mathbb{N}}$ is monotonically decreasing and bounded below by $s_{A^{\star}}(u, \alpha)$, it follows that $\left(s_{A^{\star}}\left(u, \alpha_{n}\right)\right)_{n \in \mathbb{N}}$ is convergent and that

$$
\lim _{n \rightarrow \infty} s_{A^{\star}}\left(u, \alpha_{n}\right) \geq s_{A^{\star}}(u, \alpha) .
$$

Moreover for every $n$ there exists $a_{n} \in\left[A^{\star}\right]_{\alpha_{n}}$ fulfilling $s_{A^{\star}}\left(u, \alpha_{n}\right)=\left\langle a_{n}, u\right\rangle$. Since $a_{n} \in\left[A^{\star}\right]_{\alpha_{1}} \in \mathcal{K}_{c}^{d}$ for every $n$ it follows that there exists a convergent subsequence $\left(a_{n_{j}}\right)_{n \in \mathbb{N}}$ converging to a point $a$. Since this point obviously fulfills

$$
a \in \bigcap_{j=1}^{\infty}\left[A^{\star}\right]_{\alpha_{n_{j}}}=\bigcap_{n=1}^{\infty}\left[A^{\star}\right]_{\alpha_{n}}=\left[A^{\star}\right]_{\alpha}
$$

it follows that $\lim _{n \rightarrow \infty} s_{A^{\star}}\left(u, \alpha_{n}\right)=\lim _{j \rightarrow \infty}\left\langle a_{n_{j}}, u\right\rangle=\langle a, u\rangle \leq s_{A^{\star}}(u, \alpha)$, which completes the proof of the theorem.

Furthermore it is easy to check whether a given function $f: \mathbb{R}^{d} \times(0,1] \rightarrow \mathbb{R}$ is support function of a $d$-dimensional fuzzy vector $A^{\star} \in \mathcal{F}_{c}^{d}$ :

Theorem 1.35 If a function $f: \mathbb{R}^{d} \times(0,1] \rightarrow \mathbb{R}$ is

1. subadditive and positive homogeneous in $u \in \mathbb{R}^{d}$ for fixed $\alpha \in(0,1]$ and

2. monotonically decreasing and left-continuous in $\alpha \in(0,1]$ for fixed $u \in \mathbb{R}^{d}$,

then there exists a unique fuzzy vector $A^{\star}$ such that $f$ is the support function of $A^{\star}$, i.e. $f=s_{A^{\star}}$.

Proof: Since for every fixed $\alpha \in(0,1]$ the function $f(\cdot, \alpha)$ is subadditive and positive homogeneous by assumption, applying Theorem 1.28 shows that $\left[A^{\star}\right]_{\alpha}$, defined by

$$
\left[A^{\star}\right]_{\alpha}:=\left\{a \in \mathbb{R}^{d}:\langle a, u\rangle \leq f(u, \alpha) \forall u \in S^{d-1}\right\},
$$

is a non-empty, convex compact set with support function $s_{\left[A^{\star}\right]_{\alpha}}(\cdot)=f(\cdot, \alpha)$. Furthermore it follows immediately from the second requirement that the 
family $\left(\left[A^{\star}\right]_{\alpha}\right)_{\alpha \in(0,1]}$ is a nested family, monotonically decreasing for $\alpha$ increasing. Consequently the only thing left to prove is that for every $\beta \in(0,1]$

$$
\bigcap_{\alpha<\beta}\left[A^{\star}\right]_{\alpha}=\left[A^{\star}\right]_{\beta}
$$

is fulfilled:

Since $\left[A^{\star}\right]_{\alpha} \supseteq\left[A^{\star}\right]_{\beta}$ for every $\alpha<\beta$ obviously $\bigcap_{\alpha<\beta}\left[A^{\star}\right]_{\alpha} \supseteq\left[A^{\star}\right]_{\beta}$ follows. In order to prove the opposite inclusion suppose that $a \in\left[A^{\star}\right]_{\alpha}$ for every $\alpha<\beta$. Then it follows that $\langle a, u\rangle \leq f(u, \alpha)$ for every $u \in S^{d-1}$ and every $\alpha<\beta$. Finally left-continuity of $f$ with respect to $\alpha$ implies that $\langle a, u\rangle \leq f(u, \beta)$ holds for every $u \in S^{d-1}$, which shows that $a \in\left[A^{\star}\right]_{\beta}$ and therefore completes the proof of the theorem.

Using the properties of support functions for fuzzy vectors a stronger version of Theorem 1.5 will be proved now. This theorem especially states not only that every nested monotonically decreasing family $\left(K_{\alpha}\right)_{\alpha \in(0,1]}$ in $\mathcal{K}_{c}^{d}$ induces a fuzzy vector $C^{\star} \in \mathcal{F}_{c}^{d}$ (in fact the convex hull) but also that for $\lambda$-almost every $\alpha \in(0,1]$ the equality $\left[C^{\star}\right]_{\alpha}=K_{\alpha}$ holds (compare [20]).

Theorem 1.36 Suppose that $\Lambda \subseteq(0,1]$ is a measurable set with $\lambda(\Lambda)=1$ and that $\left(K_{\alpha}\right)_{\alpha \in \Lambda}$ is a nested monotonically decreasing family of sets in $\mathcal{K}_{c}^{d}$. For every $\alpha \in(0,1]$ define a set $C_{\alpha}$ by

$$
C_{\alpha}:=\bigcap_{\{\beta: \beta \in(0, \alpha) \cap \Lambda\}} K_{\beta} .
$$

Then the family $\left(C_{\alpha}\right)_{\alpha \in(0,1]}$ is a family of $\alpha$-cuts of a fuzzy vector $C^{\star} \in \mathcal{F}_{c}^{d}$ and $\left[C^{\star}\right]_{\alpha}=C_{\alpha}=K_{\alpha}$ holds for $\lambda$-almost every $\alpha \in(0,1]$.

Proof: First of all it follows immediately from the definition of $C_{\alpha}$ that $C_{\alpha} \in \mathcal{K}_{c}^{d}$ holds for every $\alpha \in(0,1]$ and that $C_{\alpha} \supseteq C_{\beta}$ whenever $\alpha \leq \beta$. Furthermore an easy calculation shows that for every $\beta \in(0,1]$

$$
\bigcap_{\alpha<\beta} C_{\alpha}=C_{\beta}
$$

Consequently according to Theorem 1.5 the family $\left(C_{\alpha}\right)_{\alpha \in(0,1]}$ is a family of $\alpha$-cuts of a unique fuzzy vector $C^{\star} \in \mathcal{F}_{c}^{d}$, which proves the first part of the assertion in the theorem.

In order to prove the second part notice that the construction of $C_{\alpha}$ implies that for every $\beta \in \Lambda$ and $\alpha \in(0, \beta) \cap \Lambda$ the inclusion $K_{\alpha} \supseteq C_{\beta} \supseteq K_{\beta}$ holds. Define a set $D_{\alpha}$ by

$$
D_{\alpha}:=\left\{\begin{array}{ll}
K_{\alpha} & \text { if } \lambda \in \Lambda \\
C_{\alpha} & \text { otherwise }
\end{array}\right\} \forall \alpha \in(0,1]
$$


then it follows immediately that $\left(D_{\alpha}\right)_{\alpha \in(0,1]}$ is a nested family of sets in $\mathcal{K}_{c}^{d}$, that decreases monotonically in $\alpha$.

Moreover for every $v \in S^{d-1} \cap \mathbb{Q}^{d}$ define a function $g_{v}:(0,1] \rightarrow \mathbb{R}$ by

$$
g_{v}(\alpha):=s_{D_{\alpha}}(v)
$$

for every $\alpha \in(0,1]$, whereby $s_{D_{\alpha}}(\cdot)$ denotes the support function of $D_{\alpha} \in \mathcal{K}_{c}^{d}$. Since obviously $g_{v}(\cdot)$ is a monotonically decreasing function on $(0,1]$ the set $\Upsilon_{v} \subseteq(0,1]$ of all discontinuities of $g_{v}(\cdot)$ is at most countable (compare [16]). Because of the fact that $S^{d-1} \cap \mathbb{Q}^{d}$ is countable it follows that the set $\Upsilon$, defined by

$$
\Upsilon:=\bigcup_{\left\{v: v \in S^{d-1} \cap \mathbb{Q}^{d}\right\}} \Upsilon_{v}
$$

is as countable union of (at most) countable sets itself countable. Hence $\Upsilon$ is measurable and $\lambda(\Upsilon)=0$. This shows that the set $\tilde{\Lambda}$ defined by $\tilde{\Lambda}:=\Lambda \backslash \Upsilon$ is measurable and has full measure, i.e. $\lambda(\tilde{\Lambda})=1$, which in turn implies that $\tilde{\Lambda}$ is dense in $(0,1]$.

Let $\alpha \in \tilde{\Lambda}$ and $v \in S^{d-1} \cap \mathbb{Q}^{d}$ be arbitrary but fixed, then there exists a monotonically increasing sequence $\left(\alpha_{n}\right)_{n \in \mathbb{N}}$ in $(0, \alpha) \cap \tilde{\Lambda}$ that converges to $\alpha$. Because of $K_{\alpha_{n}} \supseteq C_{\alpha} \supseteq K_{\alpha}$ for all $n \in \mathbb{N}$ it follows that

$$
g_{v}\left(\alpha_{n}\right)=s_{K_{\alpha_{n}}}(v) \geq s_{C_{\alpha}}(v) \geq s_{K_{\alpha}}(v)=g_{v}(\alpha)
$$

holds for every $n \in \mathbb{N}$. Since by construction $\lim _{n \rightarrow \infty} g_{v}\left(\alpha_{n}\right)=g_{v}(\alpha)$ holds this implies that $s_{C_{\alpha}}(v)=s_{K_{\alpha}}(v)$, which in turn shows that $s_{C_{\alpha}}(v)=s_{K_{\alpha}}(v)$ holds for all $v \in S^{d-1} \cap \mathbb{Q}^{d}$ since $v$ was arbitrary. Using the fact that $S^{d-1} \cap \mathbb{Q}^{d}$ is dense in $S^{d-1}$ and the (Lipschitz-) continuity of support functions it therefore follows that $C_{\alpha}=K_{\alpha}$ is fulfilled. The fact that $\alpha \in \tilde{\Lambda}$ was arbitrary completes the proof.

Remark: Note that in case of $\Lambda=(0,1]$ the fuzzy vector $C^{\star} \in \mathcal{F}_{c}^{d}$ is exactly the convex hull as described in Theorem 1.5. Consequently starting from a nested monotonically decreasing family $\left(K_{\alpha}\right)_{\alpha \in \Lambda}$ of sets in $\mathcal{K}_{c}^{d}$ and building the convex hull $C^{\star} \in \mathcal{F}_{c}^{d}$ leaves $\lambda$-almost every $\alpha$-cut unchanged.

In the following section it will be shown how the $L^{p}$-metrics mentioned in Section 1.2 .3 can be extended to the case of fuzzy vectors. In doing so the following measurability result is needed:

Lemma 1.37 Suppose that $A^{\star} \in \mathcal{F}_{c}^{d}$ is a d-dimensional fuzzy vector with corresponding support function $s_{A^{\star}}(\cdot, \cdot)$, defined according to (1.22), and denote by $\mathcal{B}\left(S^{d-1}\right)$ and $\mathcal{B}((0,1])$ the Borel sets on $S^{d-1}$ and $(0,1]$ respectively. Then the support function $s_{A^{*}}(\cdot, \cdot)$ is measurable with respect to the product $\sigma$-algebra $\mathcal{B}\left(S^{d-1}\right) \otimes \mathcal{B}((0,1])$. 
Proof: It is well known that the family $\mathfrak{E}=\{(-\infty, t): t \in \mathbb{R}\}$ generates the Borel sets $\mathcal{B}(\mathbb{R})$ on $\mathbb{R}$. Consequently it suffices to prove that

$$
s_{A^{\star}}^{-1}((-\infty, t)) \in \mathcal{B}\left(S^{d-1}\right) \otimes \mathcal{B}((0,1])
$$

holds for every $t \in \mathbb{R}$. It follows immediately from point three in Theorem 1.34 that the following equality is fulfilled:

$$
s_{A^{\star}}^{-1}((-\infty, t))=\bigcup_{\alpha \in(0,1] \cap \mathbb{Q}}\left(\left\{u \in S^{d-1}: s_{\left[A^{\star}\right]_{\alpha}}(u)<t\right\} \times[\alpha, 1]\right)
$$

Point two of Theorem 1.34 implies that for every fixed $\alpha \in(0,1]$ the set $\left\{u \in S^{d-1}: s_{\left[A^{*}\right]_{\alpha}}(u)<t\right\}$ is an open (relative open) subset of $S^{d-1}$ and therefore Borel-measurable. Consequently (1.23) shows that $s_{A^{\star}}^{-1}((-\infty, t))$ is a countable union of measurable rectangles in $\mathcal{B}\left(S^{d-1}\right) \otimes \mathcal{B}((0,1])$ and therefore measurable, which completes the proof of the lemma.

A further measurability result will be helpful:

Lemma 1.38 For every pair $A^{\star}, B^{\star} \in \mathcal{F}_{c}^{d}$ the function $h:(0,1] \rightarrow[0, \infty)$, defined by $h(\alpha):=\delta_{H}\left(\left[A^{\star}\right]_{\alpha},\left[B^{\star}\right]_{\alpha}\right)$, is left-continuous in $\alpha$.

Proof: Suppose that $\left(\alpha_{n}\right)_{n \in \mathbb{N}}$ is a monotonically increasing sequence in $(0,1]$ that converges to $\alpha \in(0,1]$. By using Theorem 1.4 and Lemma 1.11 it follows that $\lim _{n \rightarrow \infty} \delta_{H}\left(\left[A^{\star}\right]_{\alpha_{n}},\left[A^{\star}\right]_{\alpha}\right)=0$ and $\lim _{n \rightarrow \infty} \delta_{H}\left(\left[B^{\star}\right]_{\alpha_{n}},\left[B^{\star}\right]_{\alpha}\right)=0$.

Applying the triangle inequality shows that

$\delta_{H}\left(\left[A^{\star}\right]_{\alpha},\left[B^{\star}\right]_{\alpha}\right) \leq \delta_{H}\left(\left[A^{\star}\right]_{\alpha},\left[A^{\star}\right]_{\alpha_{n}}\right)+\delta_{H}\left(\left[A^{\star}\right]_{\alpha_{n}},\left[B^{\star}\right]_{\alpha_{n}}\right)+\delta_{H}\left(\left[B^{\star}\right]_{\alpha_{n}},\left[B^{\star}\right]_{\alpha}\right)$

and

$\delta_{H}\left(\left[A^{\star}\right]_{\alpha_{n}},\left[B^{\star}\right]_{\alpha_{n}}\right) \leq \delta_{H}\left(\left[A^{\star}\right]_{\alpha_{n}},\left[A^{\star}\right]_{\alpha}\right)+\delta_{H}\left(\left[A^{\star}\right]_{\alpha},\left[B^{\star}\right]_{\alpha}\right)+\delta_{H}\left(\left[B^{\star}\right]_{\alpha},\left[B^{\star}\right]_{\alpha_{n}}\right)$.

Rearranging and combining these inequalities yields

$\left|\delta_{H}\left(\left[A^{\star}\right]_{\alpha},\left[B^{\star}\right]_{\alpha}\right)-\delta_{H}\left(\left[A^{\star}\right]_{\alpha_{n}},\left[B^{\star}\right]_{\alpha_{n}}\right)\right| \leq \delta_{H}\left(\left[A^{\star}\right]_{\alpha},\left[A^{\star}\right]_{\alpha_{n}}\right)+\delta_{H}\left(\left[B^{\star}\right]_{\alpha},\left[B^{\star}\right]_{\alpha_{n}}\right)$

which implies that

$$
\lim _{n \rightarrow \infty} \delta_{H}\left(\left[A^{\star}\right]_{\alpha_{n}},\left[B^{\star}\right]_{\alpha_{n}}\right)=\delta_{H}\left(\left[A^{\star}\right]_{\alpha},\left[B^{\star}\right]_{\alpha}\right),
$$

and therefore completes the proof of the lemma.

Denote by $\mathcal{M}\left(S^{d-1} \times(0,1], \mathbb{R}\right)$ the set of all Borel-measurable functions from $S^{d-1} \times(0,1]$ to $\mathbb{R}$. Similar to the embedding $\Phi$ treated in Theorem 1.24 according to Lemma 1.37 a mapping $\Psi: \mathcal{F}_{c}^{d} \rightarrow \mathcal{M}\left(S^{d-1} \times(0,1], \mathbb{R}\right)$ can be defined as described in the next theorem. 
Theorem 1.39 The mapping $\Psi: \mathcal{F}_{c}^{d} \rightarrow \mathcal{M}\left(S^{d-1} \times(0,1], \mathbb{R}\right)$, defined by $\Psi\left(A^{\star}\right):=s_{A^{\star}}(\cdot, \cdot)$ for every $A^{\star} \in \mathcal{F}_{c}^{d}$, has the following properties:

- $\Psi$ preserves Minkowski structure, i.e. $\Psi\left(A^{\star} \oplus B^{\star}\right)=\Psi\left(A^{\star}\right)+\Psi\left(B^{\star}\right)$ and $\Psi\left(\lambda A^{\star}\right)=\lambda \Psi\left(A^{\star}\right)$ for all $A^{\star}, B^{\star} \in \mathcal{F}_{c}^{d}$ and $\lambda \geq 0$.

- $\Psi$ preserves order, i.e. $A^{\star} \subseteq B^{\star} \Leftrightarrow s_{A^{\star}}(u, \alpha) \leq s_{B^{\star}}(u, \alpha)$ for all $(u, \alpha) \in$ $S^{d-1} \times(0,1]$ (notation according to (1.21)).

- $\Psi$ is injective.

Proof: Given $A^{\star}, B^{\star} \in \mathcal{F}_{c}^{d}$ using Theorem 1.24 it follows that

$$
\begin{aligned}
\Psi\left(A^{\star} \oplus B^{\star}\right)(u, \alpha) & =s_{A^{\star} \oplus B^{\star}}(u, \alpha)=s_{\left[A^{\star} \oplus B^{\star}\right]_{\alpha}}(u)=s_{\left[A^{\star}\right]_{\alpha}}(u)+s_{\left[B^{\star}\right]_{\alpha}}(u) \\
& =s_{A^{\star}}(u, \alpha)+s_{B^{\star}}(u, \alpha) \\
& =\Psi\left(A^{\star}\right)(u, \alpha)+\Psi\left(B^{\star}\right)(u, \alpha) \quad \forall(u, \alpha) \in S^{d-1} \times(0,1] .
\end{aligned}
$$

In completely the same manner it can be shown that

$$
\Psi\left(\lambda A^{\star}\right)(u, \alpha)=\lambda \Psi\left(A^{\star}\right)(u, \alpha)
$$

holds for all $(u, \alpha) \in S^{d-1} \times(0,1]$.

The second assertion can be proved via the following chain of equivalences (again using Theorem 1.24):

$$
\begin{aligned}
A^{\star} \subseteq B^{\star} & \Longleftrightarrow\left[A^{\star}\right]_{\alpha} \subseteq\left[B^{\star}\right]_{\alpha} \forall \alpha \in(0,1] \\
& \Longleftrightarrow s_{\left[A^{\star}\right]_{\alpha}}(u) \leq s_{\left[B^{\star}\right]_{\alpha}}(u) \forall(u, \alpha) \in S^{d-1} \times(0,1] \\
& \Longleftrightarrow s_{A^{\star}}(u, \alpha) \leq s_{B^{\star}}(u, \alpha) \forall(u, \alpha) \in S^{d-1} \times(0,1]
\end{aligned}
$$

Finally if $\Psi\left(A^{\star}\right)=\Psi\left(B^{\star}\right)$ holds for two fuzzy vectors $A^{\star}, B^{\star} \in \mathcal{F}_{c}^{d}$, then by definition $s_{\left[A^{\star}\right]_{\alpha}}(u)=s_{\left[B^{\star}\right]_{\alpha}}(u)$ is satisfied for every $\alpha \in(0,1]$ and every $u \in S^{d-1}$, which implies that $\left[A^{\star}\right]_{\alpha}=\left[B^{\star}\right]_{\alpha}$ for all $\alpha \in(0,1]$. Hence $A^{\star}=B^{\star}$ follows, which completes the proof.

\subsection{Metrics on subclasses of $\mathcal{F}_{c}^{d}$}

Starting from either the Hausdorff metric $\delta_{H}$ or the $L^{p}$-metrics $\rho_{p}$ induced by support functions on $\mathcal{K}_{c}^{d}$ various metrics can be defined on subsets of $\mathcal{F}_{c}^{d}$. First of all the set $\mathcal{F}_{c, c}^{d}$, defined by

$$
\mathcal{F}_{c, c}^{d}:=\left\{A^{\star} \in \mathcal{F}_{c}^{d}: \operatorname{supp}\left(A^{\star}\right) \text { is compact }\right\}
$$


and the following three different types of metrics on $\mathcal{F}_{c, c}^{d}$ are considered (compare [9] and [20]):

$$
\begin{gathered}
\delta_{H, \infty}^{\star}\left(A^{\star}, B^{\star}\right):=\sup _{\alpha \in(0,1]} \delta_{H}\left(\left[A^{\star}\right]_{\alpha},\left[B^{\star}\right]_{\alpha}\right) \\
\delta_{H, p}^{\star}\left(A^{\star}, B^{\star}\right):=\left(\int_{(0,1]}\left(\delta_{H}\left(\left[A^{\star}\right]_{\alpha},\left[B^{\star}\right]_{\alpha}\right)\right)^{p} d \lambda(\alpha)\right)^{1 / p} \\
\rho_{p}^{\star}\left(A^{\star}, B^{\star}\right):=\left(\int_{(0,1]} \int_{S^{d-1}}\left|s_{A^{\star}}(u, \alpha)-s_{B^{\star}}(u, \alpha)\right|^{p} d \vartheta(u) d \lambda(\alpha)\right)^{1 / p}
\end{gathered}
$$

Thereby $A^{\star}, B^{\star} \in \mathcal{F}_{c, c}^{d}, p \in[1, \infty), \lambda$ denotes the Lebesgue-measure on $(0,1]$ and $\vartheta$ denotes the normalized Lebesgue measure on $S^{d-1}$.

According to Theorem 1.24 the definitions (1.25) and (1.26) can be rewritten as

$$
\begin{gathered}
\delta_{H, \infty}^{\star}\left(A^{\star}, B^{\star}\right)=\sup _{\alpha \in(0,1]} \max _{u \in S^{d-1}}\left|s_{\left[A^{\star}\right]_{\alpha}}(u)-s_{\left[B^{\star}\right]_{\alpha}}(u)\right| \text { and } \\
\delta_{H, p}^{\star}\left(A^{\star}, B^{\star}\right)=\left(\int_{(0,1]}\left(\max _{u \in S^{d-1}}\left|s_{\left[A^{\star}\right]_{\alpha}}(u)-s_{\left[B^{\star}\right]_{\alpha}}(u)\right|\right)^{p} d \lambda(\alpha)\right)^{1 / p} .
\end{gathered}
$$

Throughout the rest of the thesis $\mathcal{L}^{p}\left(S^{d-1} \times(0,1], \vartheta \otimes \lambda\right)$ denotes the set of all measurable real-valued functions $f$, such that $|f|^{p}$ is integrable on $S^{d-1} \times(0,1]$ with respect to the product measure $\vartheta \otimes \lambda$. Furthermore for every function $f \in \mathcal{L}^{p}\left(S^{d-1} \times(0,1], \vartheta \otimes \lambda\right),\|f\|_{p}$ is defined by

$$
\|f\|_{p}=\left(\int_{S^{d-1} \times(0,1]}|f(u, \alpha)|^{p} d(\vartheta \otimes \lambda)(u, \alpha)\right)^{1 / p} .
$$

It is well known that $\|\cdot\|_{p}$ is only a seminorm on $\mathcal{L}^{p}\left(S^{d-1} \times(0,1], \vartheta \otimes \lambda\right)$, nonetheless the notation $\|\cdot\|_{p}$ will be used since no confusion will arise.

Using this notation $\rho_{p}^{\star}\left(A^{\star}, B^{\star}\right)$ is easily seen to be $\left\|s_{A^{\star}}-s_{B^{\star}}\right\|_{p}$, whereby $s_{A^{\star}}$ and $s_{B^{\star}}$ denote the corresponding support functions on $S^{d-1} \times(0,1]$, i.e.

$$
\begin{aligned}
\rho_{p}^{\star}\left(A^{\star}, B^{\star}\right) & =\left(\int_{S^{d-1} \times(0,1]}\left|s_{A^{\star}}(u, \alpha)-s_{B^{\star}}(u, \alpha)\right|^{p} d(\vartheta \otimes \lambda)(u, \alpha)\right)^{1 / p} \\
& =\left\|s_{A^{\star}}(\cdot, \cdot)-s_{B^{\star}}(\cdot, \cdot)\right\|_{p} \\
& =\left(\int_{(0,1]} \rho_{p}\left(\left[A^{\star}\right]_{\alpha},\left[B^{\star}\right]_{\alpha}\right)^{p} d \lambda(\alpha)\right)^{1 / p}
\end{aligned}
$$

whereby the last equality follows from Fubini's Theorem. 
It is straightforward to show that $\delta_{H, \infty}^{\star}(\cdot, \cdot), \delta_{H, p}^{\star}(\cdot, \cdot)$ and $\rho_{p}^{\star}(\cdot, \cdot)$ are metrics: Since for every $A^{\star} \in \mathcal{F}_{c, c}^{d}$ the corresponding support function $s_{A^{*}}(\cdot, \cdot)$ is bounded on $S^{d-1} \times(0,1]$ it follows that $\delta_{H, \infty}^{\star}\left(A^{\star}, B^{\star}\right)<\infty, \delta_{H, p}^{\star}\left(A^{\star}, B^{\star}\right)<\infty$ and $\rho_{p}^{\star}\left(A^{\star}, B^{\star}\right)<\infty$ for all $A^{\star}, B^{\star} \in \mathcal{F}_{c, c}^{d}$ (measurability of the function $\alpha \mapsto \delta_{H}\left(\left[A^{\star}\right]_{\alpha},\left[B^{\star}\right]_{\alpha}\right)$ is guaranteed by Lemma 1.38).

For $\delta_{H, \infty}^{\star}(\cdot, \cdot)$ fulfillment of the triangle inequality is an immediate consequence of the fact that $\delta_{H}$ is a metric and fulfillment of commutativity is obvious. Furthermore if $\delta_{H, \infty}^{\star}\left(A^{\star}, B^{\star}\right)=0$ then $\left[A^{\star}\right]_{\alpha}=\left[B^{\star}\right]_{\alpha}$ holds for every $\alpha \in(0,1]$, which implies that $A^{\star}=B^{\star}$. Consequently $\delta_{H, \infty}^{\star}(\cdot, \cdot)$ fulfills the conditions of a a metric on $\mathcal{F}_{c, c}^{d}$.

Concerning $\delta_{H, p}^{\star}(\cdot, \cdot)$ again commutativity is obvious and the triangle inequality is an immediate consequence of the fact that $\delta_{H}$ is a metric and the wellknown Minkowski inequality (compare [13]). Furthermore if $\delta_{H, p}^{\star}\left(A^{\star}, B^{\star}\right)=0$ then it follows that $\left[A^{\star}\right]_{\alpha}=\left[B^{\star}\right]_{\alpha}$ holds for $\lambda$-almost every $\alpha \in(0,1]$. This implies that $\left[A^{\star}\right]_{\alpha}=\left[B^{\star}\right]_{\alpha}$ for all $\alpha \in(0,1]$ since every set $M \subseteq(0,1]$ of Lebesgue measure 1 is dense in $(0,1]$. Consequently $A^{\star}=B^{\star}$ holds, which completes the proof that $\delta_{H, p}^{\star}(\cdot, \cdot)$ too is a metric on $\mathcal{F}_{c, c}^{d}$.

Finally, the fact that $\rho_{p}^{\star}(\cdot, \cdot)$ is commutative and fulfills the triangle inequality is an immediate consequence of the properties of the $L^{p}$-norm. Furthermore if $\rho_{p}^{\star}\left(A^{\star}, B^{\star}\right)=0$, then $s_{A^{\star}}(u, \alpha)=s_{B^{\star}}(u, \alpha)$ holds for $(\vartheta \otimes \lambda)$-almost every $(u, \alpha) \in S^{d-1} \times(0,1]$, which implies that there exists a set $\Lambda \subseteq(0,1]$ of Lebesgue measure 1, such that for every $\alpha \in \Lambda s_{\left[A^{\star}\right]_{\alpha}}(u)=s_{\left[B^{*}\right]_{\alpha}}(u)$ holds for $\vartheta$-almost every $u \in S^{d-1}$. Using the fact that for every set $K \in \mathcal{K}_{c}^{d}$ the corresponding support function $s_{K}(\cdot)$ is continuous on $S^{d-1}$, it follows that $\left[A^{\star}\right]_{\alpha}=\left[B^{\star}\right]_{\alpha}$ for every $\alpha \in \Lambda$. Since $\Lambda$ is a dense subset of $(0,1]$ it eventually follows that $A^{\star}=B^{\star}$.

Within the next theorems the most important properties of the three types of metrics on $\mathcal{F}_{c, c}^{d}$ are presented (compare [9] and [20]).

Theorem $1.40\left(\mathcal{F}_{c, c}^{d}, \delta_{H, \infty}^{\star}\right)$ is a complete, non-separable metric space.

Furthermore $\delta_{H, \infty}^{\star}(\cdot, \cdot)$ is translation-invariant and fulfills $\delta_{H, \infty}^{\star}\left(c A^{\star}, c B^{\star}\right)=$ $|c| \delta_{H, \infty}^{\star}\left(A^{\star}, B^{\star}\right)$ for all $A^{\star}, B^{\star} \in \mathcal{F}_{c, c}^{d}$ and $c \in \mathbb{R}$.

Proof: Completeness can most easily be proved using support functions:

Suppose that $\left(A_{n}^{\star}\right)_{n \in \mathbb{N}}$ is a Cauchy sequence in $\left(\mathcal{F}_{c, c}^{d}, \delta_{H, \infty}^{\star}\right)$. Then it follows from (1.28) and the positive homogenity of support functions for fixed $\alpha$, that for every $(u, \alpha) \in \mathbb{R}^{d} \times(0,1]$ the sequence $\left(s_{A_{n}^{\star}}(u, \alpha)\right)_{n \in \mathbb{N}}$ is a Cauchy sequence in $\mathbb{R}$ and therefore converges to a a real number denoted by $s(u, \alpha)$. By using the Cauchy property it follows that $s(\cdot, \cdot)$ is the uniform limit of $s_{A_{n}^{\star}}(\cdot, \cdot)$, i.e.

$$
\lim _{n \rightarrow \infty} \sup _{(u, \alpha) \in S^{d-1} \times(0,1]}\left|s_{A_{n}^{\star}}(u, \alpha)-s(u, \alpha)\right|=0 .
$$


Because of the fact that every function $s_{A_{n}^{*}}(\cdot, \cdot)$ is bounded on $S^{d-1} \times(0,1]$ this implies that $s(\cdot, \cdot)$ is bounded on $S^{d-1} \times(0,1]$ too.

As pointwise (in fact even uniform) limit of subadditive and positive homogeneous functions for $\alpha \in(0,1]$ fixed $s(\cdot, \alpha)$ itself is subadditive and positive homogeneous in $u \in \mathbb{R}^{d}$.

Furthermore for $u \in \mathbb{R}^{d}$ fixed $s(u, \alpha)$ as function of $\alpha$ is the uniform limit of left-continuous functions and therefore itself left-continuous in $\alpha$.

Consequently according to Theorem $1.35 \mathrm{~s}(\cdot, \cdot)$ itself is the support function of a unique $d$-dimensional fuzzy vector $A^{\star} \in \mathcal{F}_{c}^{d}$, i.e. $s(\cdot, \cdot)=s_{A^{\star}}(\cdot, \cdot)$.

From the fact that $s(\cdot, \alpha)$ is bounded on $S^{d-1} \times(0,1]$ it follows immediately that the limit $A^{\star}$ has bounded support and consequently that $A^{\star} \in \mathcal{F}_{c, c}^{d}$, which finally proves completeness.

The assertion that $\left(\mathcal{F}_{c, c}^{d}, \delta_{H, \infty}^{\star}\right)$ is not separable can easily be verified in the following way:

For $\gamma \in[0,1]$ define a $d$-dimensional fuzzy vector $A_{\gamma}^{\star} \in \mathcal{F}_{c, c}^{d}$ via its $\alpha$-cuts $\left(\left[A_{\gamma}^{\star}\right]\right)_{\alpha \in(0,1]}$ by

$$
\left[A_{\gamma}^{\star}\right]_{\alpha}:=\left\{\begin{array}{ll}
{[-1,1]^{d}} & \text { if } \alpha \leq \gamma \\
\{0\} & \text { if } \alpha>\gamma
\end{array}\right\} \forall \alpha \in(0,1] .
$$

For every pair $\gamma_{1}, \gamma_{2} \in[0,1]$ with $\gamma_{1} \neq \gamma_{2}$ obviously $\delta_{H, \infty}^{\star}\left(A_{\gamma_{1}}^{\star}, A_{\gamma_{2}}^{\star}\right)=\sqrt{d} \geq 1$ holds, which shows that there exist uncountably many fuzzy vectors in $\mathcal{F}_{c, c}^{d}$ with distance not smaller than 1 .

The remaining properties claimed are an immediate consequence of the corresponding properties of the Hausdorff metric $\delta_{H}$ stated in Proposition 1.25.

Theorem $1.41\left(\mathcal{F}_{c, c}^{d}, \delta_{H, p}^{\star}\right)$ is a non-complete, separable metric space.

Proof: Non-completeness for given $p$ can easily be demonstrated as follows: Define a sequence $\left(A_{n}^{\star}\right)_{n \in \mathbb{N}}$ in $\mathcal{F}_{c, c}^{d}$ by

$$
A_{n}^{\star}(x):=\left\{\begin{array}{ll}
1 & \text { for }\|x\|_{2} \leq 1 \\
\left(\|x\|_{2}\right)^{-2 p} & \text { for }\|x\|_{2} \in(1, n] \\
0 & \text { otherwise }
\end{array}\right\} \forall x \in \mathbb{R}^{d} .
$$

Consequently the corresponding $\alpha$-cuts are given by

$$
\left[A_{n}^{\star}\right]_{\alpha}=\left\{\begin{array}{ll}
\bar{B}\left(0, \alpha^{-1 / 2 p}\right) & \text { for } \alpha \in\left[n^{-2 p}, 1\right] \\
\bar{B}(0, n) & \text { for } \alpha \in\left(0, n^{-2 p}\right)
\end{array}\right\} \forall n \in \mathbb{N},
$$

and $\operatorname{supp}\left(A_{n}^{\star}\right)=\bar{B}(0, n)$ holds for every $n \in \mathbb{N}$.

Having that it follows that for every $n, k \in \mathbb{N}$ the following estimation holds:

$$
\delta_{H, p}^{\star}\left(A_{n}^{\star}, A_{n+k}^{\star}\right) \leq\left\{\int_{0}^{(n+k)^{-2 p}} k^{p} d \lambda(\alpha)+\int_{(n+k)^{-2 p}}^{n^{-2 p}}\left(\alpha^{-1 / 2 p}-n\right)^{p} d \lambda(\alpha)\right\}^{1 / p}
$$




$$
\begin{aligned}
& \leq\left\{\frac{k^{p}}{(n+k)^{2 p}}+\int_{(n+k)^{-2 p}}^{n^{-2 p}} \frac{1}{\sqrt{\alpha}} d \lambda(\alpha)\right\}^{1 / p} \\
& \leq\left\{\frac{1}{n^{p}}+\frac{2}{n^{p}}\right\}^{1 / p}=\frac{3^{1 / p}}{n}
\end{aligned}
$$

This shows that $\left(A_{n}^{\star}\right)_{n \in \mathbb{N}}$ is a Cauchy sequence in $\left(\mathcal{F}_{c, c}^{d}, \delta_{H, p}^{\star}\right)$. Obviously $\left(A_{n}^{\star}\right)_{n \in \mathbb{N}}$ converges with respect to $\delta_{H, p}^{\star}$ to the fuzzy vector $A^{*}$, defined by

$$
A^{\star}(x):=\left\{\begin{array}{ll}
1 & \text { for }\|x\|_{2} \leq 1 \\
\left(\|x\|_{2}\right)^{-2 p} & \text { otherwise }
\end{array}\right\} \forall x \in \mathbb{R}^{d} .
$$

Since obviously $A^{\star} \notin \mathcal{F}_{c, c}^{d}$ holds, non-completeness is proved.

In order to prove separability construct a countable family $\mathcal{Q} \subseteq \mathcal{F}_{c, c}^{d}$ as follows:

The set $\mathcal{C}$ of all d-dimensional compact cubes of the form $\times_{i=1}^{d}\left[a_{i}, b_{i}\right]$ with $a_{i}<b_{i}$ and $a_{i}, b_{i} \in \mathbb{Q}$ is countable. Consequently the set $\mathcal{C}_{s}$ of all finite unions of such cubes is countable too. Furthermore the set $\mathcal{W}$, defined by

$\mathcal{W}:=\left\{\left(\beta_{1}, \beta_{2}, \cdots, \beta_{r}\right): \beta_{i} \in \mathbb{Q} \cap(0,1]\right.$ and $\left.\beta_{1} \leq \beta_{2} \leq \cdots \leq \beta_{r}=1, r \in \mathbb{N}\right\}$,

is countable. Consequently the set $\mathcal{Q} \subseteq \mathcal{F}_{c, c}^{d}$ of all fuzzy vectors $L^{\star}$ with $\alpha$-cuts

$$
\left[L^{\star}\right]_{\alpha}=\operatorname{conv}\left(\bigcup_{\left\{i: \beta_{i} \geq \alpha\right\}} Q_{i}\right) \quad \forall \alpha \in(0,1],
$$

wherein $r \in \mathbb{N},\left(\beta_{1}, \beta_{2}, \cdots, \beta_{r}\right) \in \mathcal{W}$ and $Q_{i} \in \mathcal{C}_{s}$ for all $i \in\{1,2, \cdots, r\}$, is itself countable too.

It will be shown now that this family $\mathcal{Q}$ is dense.

In this spirit suppose that $A^{\star} \in \mathcal{F}_{c, c}^{d}$ and let $\epsilon \in(0,1)$ be arbitrary.

Since $A^{\star} \in \mathcal{F}_{c, c}^{d}$ has compact support there exists a rational number $\gamma \in \mathbb{Q}^{+}$ such that $\operatorname{supp}\left(A^{\star}\right) \subseteq[-\gamma, \gamma]^{d} \subseteq \mathbb{R}^{d}$. For every $m \in \mathbb{N}$ this cube $[-\gamma, \gamma]^{d}$ can be written as union of $m^{d}$ closed subcubes of equal edge length $\frac{2 \gamma}{m}$ with pairwise disjoint interior and vertices with rational coordinates. Choose $m$ sufficiently large, so that

$$
\frac{2 \gamma}{m}<\frac{\epsilon}{2 \sqrt{d}}
$$

holds, and denote by $Q_{1}, Q_{2}, \cdots, Q_{r}$ the set of all subcubes, that have nonempty intersection with $\operatorname{supp}\left(A^{\star}\right)$. Consequently $\operatorname{supp}\left(A^{\star}\right)$ is approximated from outside by $Q_{1}, Q_{2}, \cdots, Q_{r}$, i.e. $\bigcup_{i=1}^{r} Q_{i} \supseteq \operatorname{supp}\left(A^{\star}\right)$. Define

$$
\alpha_{i}:=\sup \left\{A^{\star}(x): x \in Q_{i}\right\}>0 \quad \text { for every } i \in\{1,2, \cdots, r\},
$$


and rename $Q_{1}, Q_{2}, \cdots, Q_{r}$ so, that $0=: \alpha_{0}<\alpha_{1} \leq \alpha_{2} \leq \cdots \leq \alpha_{r}=1$ holds. Given $\alpha \in(0,1]$ define $I_{\alpha}:=\left\{i \in\{1, \cdots, r\}: Q_{i} \cap\left[A^{\star}\right]_{\alpha} \neq \emptyset\right\}$, then $\left[A^{\star}\right]_{\alpha}$ is approximated from outside by $\bigcup_{i \in I_{\alpha}} Q_{i}$. Concerning the quality of the approximation with respect to the Hausdorff metric it follows that

$$
\begin{aligned}
\delta_{H}\left(\bigcup_{i=1}^{r} Q_{i}, \operatorname{supp}\left(A^{\star}\right)\right) & \leq \sqrt{\left(\frac{\epsilon}{2 \sqrt{d}}\right)^{2}} d=\frac{\epsilon}{2} \quad \text { and } \\
\delta_{H}\left(\bigcup_{i \in I_{\alpha}} Q_{i},\left[A^{\star}\right]_{\alpha}\right) & \leq \sqrt{\left(\frac{\epsilon}{2 \sqrt{d}}\right)^{2}} d=\frac{\epsilon}{2} .
\end{aligned}
$$

The next step is to prove that

$$
I_{\alpha}=\left\{i \in\{1, \cdots, r\}: \alpha_{i} \geq \alpha\right\}
$$

In fact, if $Q_{i_{0}} \cap\left[A^{\star}\right]_{\alpha} \neq \emptyset$, then it follows immediately that $\alpha_{i_{0}} \geq \alpha$.

On the other hand if $\alpha_{i} \geq \alpha$, then the definition of $\alpha_{i}$ implies that for every $n \in \mathbb{N}$ sufficiently large there exists $x_{n} \in Q_{i} \cap\left[A^{\star}\right]_{\alpha-1 / n}$. Since $Q_{i}$ is compact there exists a convergent subsequence $\left(x_{n_{k}}\right)_{k \in \mathbb{N}}$ of $\left(x_{n}\right)_{n \in \mathbb{N}}$ with limit $x \in Q_{i}$. As $\bigcap_{n=1}^{\infty}\left[A^{\star}\right]_{\alpha-1 / n}=\left[A^{\star}\right]_{\alpha}$ holds, it follows that $x \in\left[A^{\star}\right]_{\alpha} \cap Q_{i}$.

Having that define for every $\alpha \in(0,1]$ a non-empty compact and convex set $K_{\alpha}$ by

$$
K_{\alpha}:=\operatorname{conv}\left(\bigcup_{\left\{i: \alpha_{i} \geq \alpha\right\}} Q_{i}\right)
$$

It is obvious that $\left(K_{\alpha}\right)_{\alpha \in(0,1]}$ is a nested monotonically decreasing family in $\alpha$ of sets in $\mathcal{K}_{c}^{d}$. In addition, it follows directly from the construction that

$$
\bigcap_{\alpha<\beta} K_{\alpha}=K_{\beta}
$$

holds for every $\beta \in(0,1]$, which shows that (according to Theorem 1.5) there exists a unique fuzzy vector $K^{\star} \in \mathcal{F}_{c, c}^{d}$ with $\left[K^{\star}\right]_{\alpha}=K_{\alpha}$ for every $\alpha \in(0,1]$. If $x \in\left[A^{\star}\right]_{\alpha}$ then there exists a cube $Q_{i}$ such that $\alpha_{i} \geq \alpha$. Therefore it follows that $x \in \bigcup_{\left\{i: \alpha_{i} \geq \alpha\right\}} Q_{i}$, which shows that $\left[A^{\star}\right]_{\alpha} \subseteq\left[K^{\star}\right]_{\alpha}=K_{\alpha}$ holds for every $\alpha \in(0,1]$. This implies the following inclusion for every $\alpha \in(0,1]$ :

$$
\left[A^{\star}\right]_{\alpha} \subseteq\left[K^{\star}\right]_{\alpha}=\operatorname{conv}\left(\bigcup_{\left\{i: \alpha_{i} \geq \alpha\right\}} Q_{i}\right)=\operatorname{conv}\left(\bigcup_{i \in I_{\alpha}} Q_{i}\right)
$$

Consequently, using Lemma 1.17 and (1.31), this shows that

$$
\delta_{H}\left(\left[A^{\star}\right]_{\alpha},\left[K^{\star}\right]_{\alpha}\right)=\delta_{H}\left(\left[A^{\star}\right]_{\alpha}, \operatorname{conv}\left(\bigcup_{i \in I_{\alpha}} Q_{i}\right)\right) \leq \delta_{H}\left(\left[A^{\star}\right]_{\alpha}, \bigcup_{i \in I_{\alpha}} Q_{i}\right) \leq \frac{\epsilon}{2}
$$


for all $\alpha \in(0,1]$, which in turn implies that

$$
\delta_{H, p}^{\star}\left(A^{\star}, K^{\star}\right)=\left(\int_{(0,1]}\left(\delta_{H}\left(\left[A^{\star}\right]_{\alpha},\left[K^{\star}\right]_{\alpha}\right)\right)^{p} d \lambda(\alpha)\right)^{1 / p} \leq \frac{\epsilon}{2} .
$$

As final step in the proof the irrational $\alpha_{i}$ are approximated from below by sufficiently close rational numbers $\beta_{i}$ :

First choose an integer $M \in \mathbb{N}$ so that $M>4(r-1)^{1 / p} 2 \gamma \sqrt{d}$.

For $\alpha_{1}$ irrational choose $\beta_{1}$ so, that $\max \left\{0, \alpha_{1}-(\epsilon / M)^{p}\right\}<\beta_{1}<\alpha_{1}$, otherwise set $\beta_{1}=\alpha_{1}$. Continue inductively as follows: For $\alpha_{i+1}=\alpha_{i}$ set $\beta_{i+1}=\alpha_{i}$, for $\alpha_{i+1}>\alpha_{i}$ and $\alpha_{i+1}$ rational set $\beta_{i+1}=\alpha_{i+1}$ and for $\alpha_{i+1}>\alpha_{i}$ and $\alpha_{i+1}$ irrational choose $\beta_{i+1}$ so that $\max \left\{\alpha_{i}, \alpha_{i+1}-(\epsilon / M)^{p}\right\}<\beta_{i+1}<\alpha_{i+1}$.

This $r$ rational numbers $\beta_{1} \leq \beta_{2} \leq \cdots \leq \beta_{r}$ are used to define a fuzzy vector $L^{\star} \in \mathcal{F}_{c, c}^{d}$ by

$$
L^{\star}(x):=\left\{\begin{array}{ll}
\beta_{i} & \text { if } K^{\star}(x)=\alpha_{i} \\
0 & \text { otherwise }
\end{array}\right\} \forall x \in \mathbb{R}^{d} .
$$

Because of the fact that $\left[K^{\star}\right]_{\alpha},\left[L^{\star}\right]_{\alpha} \subseteq[-\gamma, \gamma]^{d}$ for every $\alpha \in(0,1]$ it follows immediately that $\delta_{H}\left(\left[K^{\star}\right]_{\alpha},\left[L^{\star}\right]_{\alpha}\right) \leq 2 \gamma \sqrt{d}$ for every $\alpha \in(0,1]$. Hence

$$
\begin{aligned}
\delta_{H, p}^{\star}\left(K^{\star}, L^{\star}\right) & =\left(\int_{(0,1]}\left(\delta_{H}\left(\left[K^{\star}\right]_{\alpha},\left[L^{\star}\right]_{\alpha}\right)\right)^{p} d \lambda(\alpha)\right)^{1 / p} \\
& \leq\left(\sum_{i=1}^{r-1}(2 \gamma \sqrt{d})^{p}\left(\alpha_{i}-\beta_{i}\right)\right) \\
& \leq(2 \gamma \sqrt{d})^{p}(r-1) \frac{\epsilon^{p}}{4^{p}(r-1)(2 \gamma \sqrt{d})^{p}} \leq \frac{\epsilon}{2},
\end{aligned}
$$

which together with (1.32) and the triangle inequality finally shows that $\delta_{H, p}^{\star}\left(A^{\star}, L^{\star}\right) \leq \epsilon$

Theorem $1.42\left(\mathcal{F}_{c, c}^{d}, \rho_{p}^{\star}\right)$ is a non-complete, separable metric space. Moreover for every $p \in[1, \infty)$ the metrics $\rho_{p}^{\star}$ and $\delta_{H, p}^{\star}$ induce the same topo$\log y$ on $\mathcal{F}_{c, c}^{d}$.

Proof: Using completely the same sequence $\left(A_{n}^{\star}\right)_{n \in \mathbb{N}}$ as in the proof of Theorem 1.41 it follows directly from the rotation-symmetric construction (all $\alpha$-cuts are balls) that $\delta_{H, p}^{\star}\left(A_{n}^{\star}, A_{n+k}^{\star}\right)=\rho_{p}^{\star}\left(A_{n}^{\star}, A_{n+k}^{\star}\right)$ holds for every $n, k \in \mathbb{N}$. Consequently $\left(A_{n}^{\star}\right)_{n \in \mathbb{N}}$ is also a Cauchy sequence with respect to the metric $\rho_{p}^{\star}(\cdot, \cdot)$ without limit in $\mathcal{F}_{c, c}^{d}$, which shows that $\left(\mathcal{F}_{c, c}^{d}, \rho_{p}^{\star}\right)$ is a noncomplete metric space.

The fact that the metrics $\rho_{p}^{\star}$ and $\delta_{H, p}^{\star}$ induce the same topology on $\mathcal{F}_{c, c}^{d}$ is not 
easy to prove for $d \geq 2$, although at first sight inequality (1.17) by Vitale may seem strong enough. In their book [9] Diamond and Kloeden actually deduce that $\rho_{p}^{\star}$ and $\delta_{H, p}^{\star}$ induce the same topology on $\mathcal{F}_{c, c}^{d}$ by simply using (1.17) without giving precise arguments. Fortunately using some results from measure theory an even stronger result by Krätschmer [20] stated in Theorem 1.46 can be proved, which immediately yields that $\rho_{p}^{\star}$ and $\delta_{H, p}^{\star}$ induce the same topology on $\mathcal{F}_{c, c}^{d}$.

Given this fact, separability of $\left(\mathcal{F}_{c, c}^{d}, \rho_{p}^{\star}\right)$ immediately follows from Theorem 1.41 .

Having in mind firstly the well-known completeness of $L^{p}$-spaces in measure theory, secondly the non-completeness stated in Theorem 1.41 and Theorem 1.42 and thirdly equation (1.30) it seems reasonable to extend the metric $\rho_{p}^{\star}$ from $\mathcal{F}_{c, c}^{d}$ to the set $\mathcal{F}_{c, p}^{d}$ of all fuzzy vectors with $p$-integrable support functions on $S^{d-1} \times(0,1]$ with respect to the product measure $\vartheta \otimes \lambda$, i.e.

$$
\mathcal{F}_{c, p}^{d}:=\left\{A^{\star} \in \mathcal{F}_{c}^{d}:\left\|s_{A^{\star}}(\cdot, \cdot)\right\|_{p}<\infty\right\}
$$

Thereby, as before, $\left\|s_{A^{\star}}(\cdot, \cdot)\right\|_{p}$ is defined by

$$
\left\|s_{A^{\star}}(\cdot, \cdot)\right\|_{p}=\left(\int_{S^{d-1} \times(0,1]}\left|s_{A^{\star}}(u, \alpha)\right|^{p} d(\vartheta \otimes \lambda)(u, \alpha)\right)^{1 / p} .
$$

The following lemma shows that not only the extension of $\rho_{p}^{\star}$ but also the extension of $\delta_{H, p}^{\star}$ to $\mathcal{F}_{c, p}^{d}$ is possible.

Lemma 1.43 For every $p \in[1, \infty) \rho_{p}^{\star}(\cdot, \cdot)$ and $\delta_{H, p}^{\star}(\cdot, \cdot)$ are metrics on $\mathcal{F}_{c, p}^{d}$. Moreover $\rho_{p}^{\star}\left(A^{\star}, B^{\star}\right) \leq \delta_{H, p}^{\star}\left(A^{\star}, B^{\star}\right)$ holds for arbitrary $A^{\star}, B^{\star} \in \mathcal{F}_{c, p}^{d}$.

Proof: $\rho_{p}^{\star}(\cdot, \cdot)$ is easily seen to be a metric on $\mathcal{F}_{c, p}^{d}$ :

It follows immediately from the definition that for every pair $A^{\star}, B^{\star} \in \mathcal{F}_{c, p}^{d}$ $0 \leq \rho_{p}^{\star}\left(A^{\star}, B^{\star}\right)<\infty$ holds, that the triangle inequality is fulfilled and that $\rho_{p}^{\star}(\cdot, \cdot)$ is commutative. If $\rho_{p}^{\star}\left(A^{\star}, B^{\star}\right)=0$ then according to equation (1.30) there exists a measurable set $\Lambda \subseteq(0,1]$ with $\alpha(\Lambda)=1$ such that for every $\alpha \in \Lambda s_{\left[A^{\star}\right]_{\alpha}}(u)=s_{\left[B^{\star}\right]_{\alpha}}(u)$ holds for $\vartheta$-almost every $u \in S^{d-1}$.

Using continuity of support functions for $\alpha$ fixed this implies that for every $\alpha \in \Lambda$ the equality $s_{\left[A^{*}\right]_{\alpha}}(u)=s_{\left[B^{*}\right]_{\alpha}}(u)$ is fulfilled for every $u \in S^{d-1}$, which shows that $\left[A^{\star}\right]_{\alpha}=\left[B^{\star}\right]_{\alpha}$ for every $\alpha \in \Lambda$. Since $\Lambda$ is as set of full measure a dense subset of $(0,1]$, it follows that $\left[A^{\star}\right]_{\alpha}=\left[B^{\star}\right]_{\alpha}$ for every $\alpha \in(0,1]$ and therefore that $A^{\star}=B^{\star}$, which completes the proof that $\rho_{p}^{\star}(\cdot, \cdot)$ is a metric on $\mathcal{F}_{c, p}^{d}$.

Concerning $\delta_{H, p}^{\star}(\cdot, \cdot)$ the first thing to prove is that $\delta_{H, p}^{\star}\left(A^{\star}, B^{\star}\right)<\infty$ for 
every pair $A^{\star}, B^{\star} \in \mathcal{F}_{c, p}^{d}$ :

For $d=1$ this is trivial since the metrics $\delta_{H}$ and $\rho_{p}$ are equivalent as mentioned before.

For $d \geq 2$ define the function $h:(0,1] \rightarrow[0, \infty)$ by $h(\alpha)=\delta_{H}\left(\left[A^{\star}\right]_{\alpha},\left[B^{\star}\right]_{\alpha}\right)$ for every $\alpha \in(0,1]$ as in Lemma 1.38 .

Looking back at inequality $(1.18)$, since $\operatorname{diam}(\{0\})=0$, it follows that

$$
\begin{aligned}
& \delta_{H}\left(\left[A^{\star}\right]_{\alpha},\{0\}\right) \leq \frac{2}{C(d, p)} \rho_{p}\left(\left[A^{\star}\right]_{\alpha},\{0\}\right) \text { and } \\
& \delta_{H}\left(\left[B^{\star}\right]_{\alpha},\{0\}\right) \leq \frac{2}{C(d, p)} \rho_{p}\left(\left[B^{\star}\right]_{\alpha},\{0\}\right),
\end{aligned}
$$

which implies that

$$
\begin{aligned}
h(\alpha) & =\delta_{H}\left(\left[A^{\star}\right]_{\alpha},\left[B^{\star}\right]_{\alpha}\right) \leq \delta_{H}\left(\left[A^{\star}\right]_{\alpha},\{0\}\right)+\delta_{H}\left(\{0\},\left[B^{\star}\right]_{\alpha}\right) \\
& \leq \frac{2}{C(d, p)} \rho_{p}\left(\left[A^{\star}\right]_{\alpha},\{0\}\right)+\frac{2}{C(d, p)} \rho_{p}\left(\left[B^{\star}\right]_{\alpha},\{0\}\right)
\end{aligned}
$$

for every $\alpha \in(0,1]$. Having that it follows from (1.26) and the Minkowski inequality that

$$
\begin{aligned}
\delta_{H, p}^{\star}\left(A^{\star}, B^{\star}\right)= & \left(\int_{(0,1]}(h(\alpha))^{p} d \lambda(\alpha)\right)^{1 / p} \\
\leq & \frac{2}{C(d, p)}\left\{\left(\int_{(0,1]}\left(\rho_{p}\left(\left[A^{\star}\right]_{\alpha},\{0\}\right)\right)^{p} d \lambda(\alpha)\right)^{1 / p}+\right. \\
& \left.\quad+\left(\int_{(0,1]}\left(\rho_{p}\left(\left[B^{\star}\right]_{\alpha},\{0\}\right)\right)^{p} d \lambda(\alpha)\right)^{1 / p}\right\} \\
= & \frac{2}{C(d, p)}\left\{\left\|s_{A^{\star}}(\cdot, \cdot)\right\|_{p}+\left\|s_{B^{\star}}(\cdot, \cdot)\right\|_{p}\right\}<\infty .
\end{aligned}
$$

Moreover looking at (1.16) it is obvious that for every pair $A^{\star}, B^{\star} \in \mathcal{F}_{c, p}^{d}$

$$
\rho_{p}^{\star}\left(A^{\star}, B^{\star}\right) \leq \delta_{H, p}^{\star}\left(A^{\star}, B^{\star}\right)
$$

holds.

The fact that $\delta_{H, p}^{\star}(\cdot, \cdot)$ fulfills the triangle inequality and that $\delta_{H, p}^{\star}(\cdot, \cdot)$ is commutative follows immediately from the definition and the fact that $\delta_{H}$ is a metric on $\mathcal{K}_{c}^{d}$. Finally $\delta_{H, p}^{\star}\left(A^{\star}, B^{\star}\right)=0$ according to (1.34) implies that $\rho_{p}^{\star}\left(A^{\star}, B^{\star}\right)=0$, which shows that $A^{\star}=B^{\star}$. This completes the proof of the theorem.

Theorem $1.44 \mathcal{F}_{c, c}^{d}$ is dense in $\mathcal{F}_{c, p}^{d}$ with respect to $\delta_{H, p}^{\star}(\cdot, \cdot)$ and with respect to $\rho_{p}^{\star}(\cdot, \cdot)$ for every $p \in[1, \infty)$. 
Proof: Suppose that $A^{\star} \in \mathcal{F}_{c, p}^{d}$ is given. It will be shown now that there exists a sequence $\left(A_{n}^{\star}\right)_{n \in \mathbb{N}}$ of sets in $\mathcal{F}_{c, c}^{d}$ that converges to $A^{\star}$ with respect to the metric $\delta_{H, p}^{\star}(\cdot, \cdot)$. According to $(1.34)$ this implies that $\left(A_{n}^{\star}\right)_{n \in \mathbb{N}}$ also converges to $A^{\star}$ with respect to the metric $\rho_{p}^{\star}(\cdot, \cdot)$.

For every $n \in \mathbb{N}$ define $A_{n}^{\star} \in \mathcal{F}_{c, c}^{d}$ by

$$
A_{n}^{\star}(x):=\left\{\begin{array}{ll}
A^{\star}(x) & \text { if } A^{\star}(x) \geq \frac{1}{n} \\
0 & \text { otherwise }
\end{array}\right\} \quad \forall x \in \mathbb{R}^{d},
$$

which implies that

$$
\left[A_{n}^{\star}\right]_{\alpha}=\left\{\begin{array}{ll}
{\left[A^{\star}\right]_{\alpha}} & \text { for } \alpha \in\left[\frac{1}{n}, 1\right] \\
{\left[A^{\star}\right]_{1 / n}} & \text { for } \alpha \in\left(0, \frac{1}{n}\right)
\end{array}\right\} \subseteq\left[A^{\star}\right]_{\alpha} \quad \forall \alpha \in(0,1] .
$$

For every $n \in \mathbb{N}$ define a function $h_{n}:(0,1] \rightarrow[0, \infty)$ by

$$
h_{n}(\alpha):=\left(\delta_{H}\left(\left[A_{n}^{\star}\right]_{\alpha},\left[A^{\star}\right]_{\alpha}\right)^{p}\right.
$$

for every $\alpha \in(0,1]$. It follows immediately from the construction that $\lim _{n \rightarrow \infty} h_{n}(\alpha)=0$ for every $\alpha \in(0,1]$.

Furthermore again by using (1.18) it follows that

$$
h_{n}(\alpha) \leq 2^{p} \delta_{H}\left(\left[A^{\star}\right]_{\alpha},\{0\}\right)^{p} \leq\left(\frac{4}{C(d, p)}\right)^{p} \rho_{p}\left(\left[A^{\star}\right]_{\alpha},\{0\}\right)^{p} \quad \forall \alpha \in(0,1] .
$$

Since by assumption $A^{\star} \in \mathcal{F}_{c, p}^{d}$ it follows from Fubini's Theorem that the function $\alpha \mapsto \rho_{p}\left(\left[A^{\star}\right]_{\alpha},\{0\}\right)^{p}$ is integrable over $(0,1]$.

Consequently applying Lebesgue's Dominated Convergence Theorem proves that $\lim _{n \rightarrow \infty} \delta_{H, p}^{\star}\left(A_{n}^{\star}, A^{\star}\right)=0$.

It will be proved now that for every (fixed) $p \geq 1$ the metrics $\rho_{p}^{\star}(\cdot, \cdot)$ and $\delta_{H, p}^{\star}(\cdot, \cdot)$ induce the same topology on $\mathcal{F}_{c, p}^{d}$. As a first step going in that direction inequality (1.34) implies that if a sequence $\left(A_{n}^{\star}\right)_{n \in \mathbb{N}}$ in $\mathcal{F}_{c, p}^{d}$ converges to $A^{\star} \in \mathcal{F}_{c, p}^{d}$ with respect to $\delta_{H, p}^{\star}$, then it particularly converges to $A^{\star}$ with respect to $\rho_{p}^{\star}$ too.

The main tool for proving the opposite implication is a well known theorem from measure theory quoted as Theorem 6.14 in the Appendix. This approach goes back to Krätschmer [20], however the proofs below are shorter than the original ones and produce the same result.

Lemma 1.45 Suppose that $\left(A_{n}^{\star}\right)_{n \in \mathbb{N}}$ is a sequence in $\mathcal{F}_{c, p}^{d}$ that converges to $A^{\star} \in \mathcal{F}_{c, p}^{d}$ with respect to the metric $\rho_{p}^{\star}(\cdot, \cdot)$. Then it converges to $A^{\star}$ with respect to the metric $\delta_{H, p}^{\star}(\cdot, \cdot)$ too. 
Proof: Suppose that $\left(A_{n}^{\star}\right)_{n \in \mathbb{N}}$ is a sequence in $\mathcal{F}_{c, p}^{d}$ that converges to $A^{\star} \in \mathcal{F}_{c, p}^{d}$ with respect to the metric $\rho_{p}^{\star}(\cdot, \cdot)$.

For every $n \in \mathbb{N}$ define a function $h_{n}:(0,1] \rightarrow[0, \infty)$ by

$$
h_{n}(\alpha):=\delta_{H}\left(\left[A_{n}^{\star}\right]_{\alpha},\left[A^{\star}\right]_{\alpha}\right) \quad \forall \alpha \in(0,1] .
$$

Again using the same estimation as in the proof of Lemma 1.43 with $A_{n}^{\star}$ instead of $B^{\star}$ and the triangle inequality, it follows that

$$
\begin{aligned}
h_{n}(\alpha) & \leq \frac{2}{C(d, p)}\left(\rho_{p}\left(\left[A_{n}^{\star}\right]_{\alpha},\{0\}\right)+\rho_{p}\left(\left[A^{\star}\right]_{\alpha},\{0\}\right)\right) \\
& \leq \frac{2}{C(d, p)}\left(2 \rho_{p}\left(\left[A_{n}^{\star}\right]_{\alpha},\{0\}\right)+\rho_{p}\left(\left[A_{n}^{\star}\right]_{\alpha},\left[A^{\star}\right]_{\alpha}\right)\right) .
\end{aligned}
$$

For every $\alpha \in(0,1]$ and every $n \in \mathbb{N}$ set

$$
g_{n}(\alpha):=2 \rho_{p}\left(\left[A_{n}^{\star}\right]_{\alpha},\{0\}\right)+\rho_{p}\left(\left[A_{n}^{\star}\right]_{\alpha},\left[A^{\star}\right]_{\alpha}\right) .
$$

Using Fubini's Theorem it follows that $g_{n}(\cdot)$ has as sum of two measurable functions with finite $p$-mean over $(0,1]$ itself finite $p$-mean, i.e.

$$
\int_{(0,1]}\left(g_{n}(\alpha)\right)^{p} d \lambda(\alpha)<\infty \quad \forall n \in \mathbb{N} .
$$

Since by assumption $\lim _{n \rightarrow \infty} \rho_{p}^{\star}\left(A^{\star}, A_{n}^{\star}\right)=0$ holds, again by using Fubini's Theorem this implies that the sequence $g_{n}(\cdot)$ converges in $p$-mean to (the measurable function) $g(\alpha)=2 \rho_{p}\left(\left[A^{\star}\right]_{\alpha},\{0\}\right)$. Consequently by Theorem 6.14 in the Appendix it follows immediately that the sequence $\left(g_{n}^{p}\right)_{n \in \mathbb{N}}$ is uniformly integrable, which shows that the sequence $\left(h_{n}^{p}\right)_{n \in \mathbb{N}}$ is uniformly integrable since

$$
h_{n}(\alpha) \leq \frac{2}{C(d, p)} g_{n}(\alpha)
$$

holds for every $\alpha \in(0,1]$ and every $n \in \mathbb{N}$.

In order to be able to apply Theorem 6.14 it is necessary to prove that $h_{n}(\cdot)$ converges to 0 in measure. For that purpose suppose that $\left(h_{n_{k}}\right)_{k \in \mathbb{N}}$ is a subsequence of $\left(h_{n}\right)_{n \in \mathbb{N}}$. Then surely $\lim _{k \rightarrow \infty} \rho_{p}^{\star}\left(A_{n_{k}}^{\star}, A^{\star}\right)=0$ holds, which implies that there exists a further subsequence $\left(n_{k_{j}}\right)_{j \in \mathbb{N}}$ of $\left(n_{k}\right)_{k \in \mathbb{N}}$, such that

$$
\lim _{j \rightarrow \infty} s_{A_{n_{k_{j}}}^{\star}}(u, \alpha)=s_{A^{\star}}(u, \alpha)
$$

for $(\vartheta \otimes \lambda)$-almost every $(u, \alpha) \in S^{d-1} \times(0,1]$. Consequently there exists a measurable set $\Lambda \subseteq(0,1]$ with $\lambda(\Lambda)=1$ such that for every $\alpha \in \Lambda$ $\lim _{j \rightarrow \infty} s_{A_{n_{k_{j}}}^{\star}}(u, \alpha)=s_{A^{\star}}(u, \alpha)$ holds for $\vartheta$-almost every $u \in S^{d-1}$. Applying Theorem 1.30 this shows that $\lim _{j \rightarrow \infty} h_{n_{k_{j}}}(\alpha)=0$ for $\lambda$-almost every 
$\alpha \in(0,1]$. Moreover according to Theorem 6.15 this shows that $h_{n}(\cdot)$ converges to 0 in measure.

Finally applying Theorem 6.14 this shows that

$$
\lim _{n \rightarrow \infty}\left(\delta_{H, p}^{\star}\left(A^{\star}, A_{n}^{\star}\right)\right)^{p}=\lim _{n \rightarrow \infty} \int_{(0,1]}\left(h_{n}(\alpha)\right)^{p} d \lambda(\alpha)=0,
$$

which completes the proof.

Theorem 1.46 For every fixed $p \in[1, \infty)$ the metrics $\rho_{p}^{\star}$ and $\delta_{H, p}^{\star}$ induce the same topology on $\mathcal{F}_{c, p}^{d}$.

Proof: According to Lemma 1.45 and inequality (1.34) it follows immediately that a sequence $\left(A_{n}^{\star}\right)_{n \in \mathbb{N}}$ in $\mathcal{F}_{c, p}^{d}$ converges to $A^{\star} \in \mathcal{F}_{c, p}^{d}$ with respect to the metric $\rho_{p}^{\star}(\cdot, \cdot)$ if and only if it converges to $A^{\star}$ with respect to the metric $\delta_{H, p}^{\star}(\cdot, \cdot)$. Consequently a set $\mathcal{C} \subseteq \mathcal{F}_{c, p}^{d}$ is closed with respect to $\rho_{p}^{\star}(\cdot, \cdot)$ if and only if it is closed with respect to $\delta_{H, p}^{\star}(\cdot, \cdot)$. This proves that the topologies induced by the metrics are the same.

Finally the main Theorem of this section can be stated and proved (again compare [20]):

Theorem 1.47 For every $p \in[1, \infty)$ both $\left(\mathcal{F}_{c, p}^{d}, \rho_{p}^{\star}\right)$ and $\left(\mathcal{F}_{c, p}^{d}, \delta_{H, p}^{\star}\right)$ are complete separable metric spaces.

Proof: Separability of $\left(\mathcal{F}_{c, p}^{d}, \delta_{H, p}^{\star}\right)$ is an immediate consequence of Theorem 1.41 and Theorem 1.44. Having that separability of $\left(\mathcal{F}_{c, p}^{d}, \rho_{p}^{\star}\right)$ follows immediately from Theorem 1.46 .

Furthermore according to (1.34) and Lemma 1.45 completeness of $\left(\mathcal{F}_{c, p}^{d}, \rho_{p}^{\star}\right)$ implies completeness of $\left(\mathcal{F}_{c, p}^{d}, \delta_{H, p}^{\star}\right)$. Consequently it suffices to prove that $\left(\mathcal{F}_{c, p}^{d}, \rho_{p}^{\star}\right)$ is a complete metric space.

Therefore suppose that $\left(A_{n}^{\star}\right)_{n \in \mathbb{N}}$ is a Cauchy sequence in $\mathcal{F}_{c, p}^{d}$ with respect to $\rho_{p}^{\star}(\cdot, \cdot)$. Because of equality $(1.30)$ it follows that $\left(s_{A_{n}^{\star}}\right)_{n \in \mathbb{N}}$ is a Cauchy sequence in $\mathcal{L}^{p}\left(S^{d-1} \times(0,1], \vartheta \otimes \lambda\right)$. Hence (compare [1]) there exists a realvalued function $f \in \mathcal{L}^{p}\left(S^{d-1} \times(0,1], \vartheta \otimes \lambda\right)$ such that

$$
\lim _{n \rightarrow \infty}\left\|s_{A_{n}^{\star}}-f\right\|_{p}=0
$$

and such that there exists a subsequence $\left(s_{A_{n_{j}}^{\star}}\right)_{j \in \mathbb{N}}$ with

$$
\lim _{j \rightarrow \infty} s_{A_{n_{j}}^{\star}}(u, \alpha)=f(u, \alpha)
$$


for $(\vartheta \otimes \lambda)$-almost every $(u, \alpha) \in S^{d-1} \times(0,1]$. Using $(1.36)$ it follows immediately that there exists a exists a measurable set $\Lambda \subseteq(0,1]$ fulfilling $\lambda(\Lambda)=1$, such that for every $\alpha \in \Lambda$

$$
\lim _{j \rightarrow \infty} s_{A_{n_{j}}^{\star}}(u, \alpha)=\lim _{j \rightarrow \infty} s_{\left[A_{n_{j}}^{\star}\right]_{\alpha}}(u, \alpha)=f(u, \alpha)
$$

holds for $\vartheta$-almost every $u \in S^{d-1}$. Consequently Theorem 1.29 and Theorem 1.30 together imply that for every $\alpha \in \Lambda$ the sequence $s_{A_{n_{j}}^{*}}(u, \alpha)$ converges to $f(u, \alpha)$ uniformly in $u \in S^{d-1}$ and that for every $\alpha \in \Lambda$ there exists a set $K_{\alpha} \in \mathcal{K}_{c}^{d}$, such that $s_{K_{\alpha}}(u)=f(u, \alpha)$ holds for every $u \in S^{d-1}$.

Using (1.37) and the fact that for every $A_{n}^{\star}$ the family $\left(\left[A_{n}^{\star}\right]_{\alpha}\right)_{\alpha \in(0,1]}$ of its $\alpha$-cuts decreases in $\alpha$, it follows immediately that for every $u \in S^{d-1}$ and every $\alpha, \beta \in \Lambda f(u, \alpha) \geq f(u, \beta)$ holds whenever $\alpha \leq \beta$. This directly proves that $K_{\alpha} \supseteq K_{\beta}$ whenever $\alpha, \beta \in \Lambda$ and $\alpha \leq \beta$.

Therefore $\left(K_{\alpha}\right)_{\alpha \in \Lambda}$ is a family in $\mathcal{K}_{c}^{d}$ that decreases monotonically in $\alpha$, which allows to apply Theorem 1.36 to this situation. Consequently there exists a fuzzy vector $C^{\star} \in \mathcal{F}_{c}^{d}$, such that $\left[C^{\star}\right]_{\alpha}=K_{\alpha}$ for $\lambda$-almost every $\alpha \in(0,1]$, which in turn shows that

$$
s_{C^{\star}}(u, \alpha)=f(u, \alpha)
$$

holds for $\vartheta \otimes \lambda$-almost every $(u, \alpha) \in S^{d-1} \times(0,1]$.

Since $f \in \mathcal{L}^{p}\left(S^{d-1} \times(0,1], \vartheta \otimes \lambda\right)$ it follows directly from (1.38) that $s_{C^{\star}} \in$ $\mathcal{L}^{p}\left(S^{d-1} \times(0,1], \vartheta \otimes \lambda\right)$, which means that $C^{\star} \in \mathcal{F}_{c, p}^{d}$. Moreover looking at (1.35) equation (1.38) implies that

$$
\lim _{n \rightarrow \infty} \rho_{p}^{\star}\left(A_{n}^{\star}, C^{\star}\right)=\lim _{n \rightarrow \infty}\left\|s_{A_{n}^{\star}}-s_{C^{\star}}\right\|_{p}=0 .
$$

This completes the proof. 


\section{Chapter 2}

\section{Fuzzy probability distributions}

\subsection{Motivation}

As mentioned at the beginning of the previous chapter the unavoidable fuzziness (imprecision) of measurements of one-dimensional continuous quantities can be modelled by the concept of fuzzy numbers. Concerning probability theory and statistics this fuzziness must not be neglected and has far-reaching consequences as will be demonstrated below.

From the classical point of view one of the most central results of probability theory is the well-known Strong Law of Large Numbers (SLLN), basically going back to Komogorov in 1930. The version stated below goes back to Etemadi (compare [3] and [14]):

Theorem 2.1 (SLLN) Let $X, X_{1}, X_{2}, \cdots$ be identically distributed, pairwise independent, integrable random variables on a probability space $(\Omega, \mathcal{A}, \mathcal{P})$. Then

$$
\lim _{n \rightarrow \infty} \frac{1}{n} \sum_{i=1}^{n} X_{i}(\omega)=\mathbb{E}(X)=\int_{\Omega} X(\omega) d \mathcal{P}(\omega)
$$

holds for $\mathcal{P}$-almost every $\omega \in \Omega$.

If $\left(X_{n}\right)_{n \in \mathbb{N}}$ fulfills the conditions of Theorem 2.1 and $B \in \mathcal{B}(\mathbb{R})$ is a Borel set, then obviously the sequence $\left(Y_{n}\right)_{n \in \mathbb{N}}$ of $\{0,1\}$-valued random variables, defined by

$$
Y_{n}(\omega):=\mathbf{1}_{B} \circ X_{n}(\omega)
$$

for every $n \in \mathbb{N}$ and every $\omega \in \Omega$, also fulfills the conditions of Theorem 2.1. Define the relative frequency $h_{n}(B, \omega)$ of the set $B$ with respect to $X_{1}(\omega), \cdots, X_{n}(\omega)$ for every $n \in \mathbb{N}$ and every $\omega \in \Omega$ by

$$
h_{n}(B, \omega):=\frac{1}{n} \#\left\{i \in\{1,2, \cdots, n\}: X_{i}(\omega) \in B\right\} .
$$


Then according to Theorem 2.1 for $\mathcal{P}$-almost every $\omega \in \Omega$ the following equality holds

$$
\begin{aligned}
\lim _{n \rightarrow \infty} h_{n}(B, \omega) & =\lim _{n \rightarrow \infty} \frac{1}{n} \sum_{i=1}^{n} Y_{i}(\omega)=\int_{\Omega} 1_{B} \circ X(\omega) d \mathcal{P}(\omega) \\
& =\mathcal{P}(\{\omega \in \Omega: X(\omega) \in B\})=\mathcal{P}^{X}(B),
\end{aligned}
$$

whereby $\mathcal{P}^{X}$ denotes the distribution of the random variable $X$.

In other words: For every Borel set $B \in \mathcal{B}(\mathbb{R})$ there exists a set $M \in \mathcal{A}$ with $P(M)=1$, such that the probability of the event $\{X \in B\}$ is exactly the limit of the relative frequencies of the set $B$ for every $\omega \in M$. Particularly, with probability 1 probabilities of events can be approximated by relative frequencies of (sufficiently large) samples $x_{1}, \ldots, x_{n}$.

In the realistic case that the samples consist in fuzzy numbers $x_{1}^{\star}, \ldots, x_{n}^{\star}$ the first problem that arises is, how the concept of relative frequencies can be extended from the idealized case of real samples. The intuitive best approach is based on so-called hitting and missing sets from the theory of random sets (for random sets compare [27] and [29]) and works as follows:

Suppose that $B \subseteq \mathbb{R}$ and that $x_{1}^{\star}, x_{2}^{\star}, \ldots, x_{n}^{\star}$ is a fuzzy sample. As usual for every $\alpha \in(0,1]$ and every $i \in\{1, \ldots, n\}$ let $\left[x_{i}^{\star}\right]_{\alpha}$ denote the $\alpha$-cut of the fuzzy number $x_{i}^{\star}$.

For every $\alpha \in(0,1]$ the lower relative frequency of level $\alpha$, denoted by $\underline{h}_{n, \alpha}(B)$, and the upper relative frequency of level $\alpha$, denoted by $\bar{h}_{n, \alpha}(B)$, are defined by

$$
\begin{aligned}
\underline{h}_{n, \alpha}(B) & :=\frac{1}{n} \#\left\{i \in\{1, \ldots, n\}:\left[x_{i}^{\star}\right]_{\alpha} \subseteq B\right\} \\
\bar{h}_{n, \alpha}(B) & :=\frac{1}{n} \#\left\{i \in\{1, \ldots, n\}:\left[x_{i}^{\star}\right]_{\alpha} \cap B \neq \emptyset\right\} .
\end{aligned}
$$

Thus the lower relative frequency of level $\alpha$ counts all $i \in\{1,2, \cdots, n\}$ for which the $\alpha$-cut of $x_{i}^{\star}$ is contained in the set $B$ and divides by $n$, whereas the upper relative frequency of level $\alpha$ counts all $i \in\{1,2, \cdots, n\}$ for which the $\alpha$-cut of $x_{i}^{\star}$ has non-empty intersection with the set $B$ and divides by $n$.

Since obviously $\underline{h}_{n, \alpha}(B) \leq \bar{h}_{n, \alpha}(B)$ holds for every $n \in \mathbb{N}$, for every $\alpha \in(0,1]$ and every $B \in \mathcal{B}$, it follows immediately that $\left(\left[\underline{h}_{n, \alpha}(B), \bar{h}_{n, \alpha}(B)\right]\right)_{\alpha \in(0,1]}$ is a family of compact non-empty intervals (for $n$ and $B$ fixed) in $\alpha$.

Furthermore again for $n$ and $B$ fixed it follows immediately from the definition that $\underline{h}_{n, \alpha}(B)$ increases if $\alpha$ increases and that $\bar{h}_{n, \alpha}(B)$ decreases if 
$\alpha$ increases. Consequently $\left(\left[\underline{h}_{n, \alpha}(B), \bar{h}_{n, \alpha}(B)\right]\right)_{\alpha \in(0,1]}$ is a family of compact non-empty intervals for $n$ and $B$ fixed, that decreases if $\alpha$ increases, i.e.

$$
\left[\underline{h}_{n, \alpha}(B), \bar{h}_{n, \alpha}(B)\right] \supseteq\left[\underline{h}_{n, \beta}(B), \bar{h}_{n, \beta}(B)\right]
$$

holds for $\alpha \leq \beta$ and $\alpha, \beta \in(0,1]$.

It can be shown easily (by a small modification of Example 4.21 in Section 4.3 below) that unfortunately in general $\left(\left[\underline{h}_{n, \alpha}(B), \bar{h}_{n, \alpha}(B)\right]\right)_{\alpha \in(0,1]}$ is not a family of $\alpha$-cuts of a fuzzy number, but at least there exists a fuzzy number (in fact the convex hull), denoted by $h_{n}^{\star}(B) \in \mathcal{F}_{c}^{1}$, such that

$$
\left[h_{n}^{\star}(B)\right]_{\alpha}=\left[\underline{h}_{n, \alpha}(B), \bar{h}_{n, \alpha}(B)\right]
$$

holds for all except (at most) finitely many $\alpha \in(0,1]$. This fuzzy number $h_{n}^{\star}(B) \in \mathcal{F}_{c}^{1}$ will be called the fuzzy relative frequency of the set $B$ with respect to the sample $x_{1}^{\star}, x_{2}^{\star}, \ldots, x_{n}^{\star}$.

Figure 2.1: Fuzzy sample of size 10

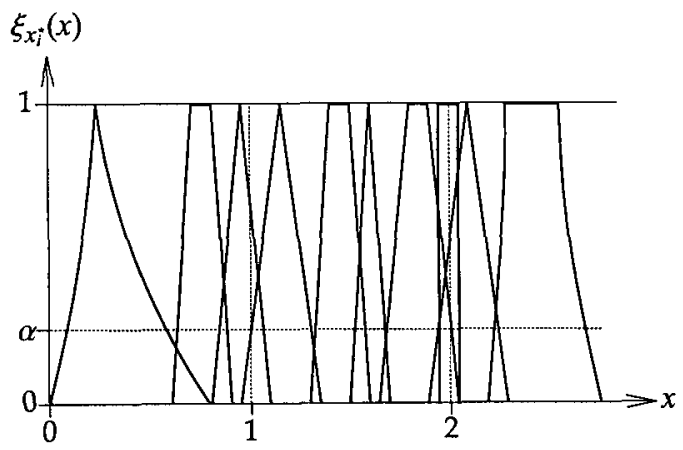

Figure 2.2: Fuzzy relative frequency $h_{10}^{\star}([1,2])$

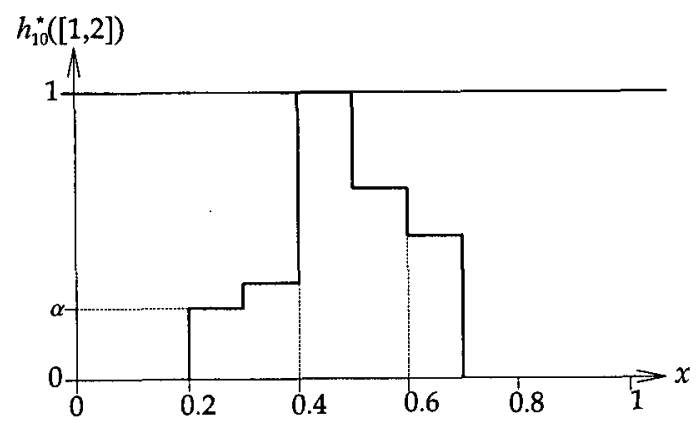


Note that if $x_{1}^{\star}, x_{2}^{\star}, \ldots, x_{n}^{\star}$ is a real sample, then $h_{n}^{\star}(B)$ coincides with the indicator function of the classical relative frequency, i.e. $h_{n}^{\star}(B)=1_{\left\{h_{n}(B)\right\}}$, which shows that this notion of fuzzy relative frequency really is a generalization of the classical concept.

For $n \in \mathbb{N}$ fixed this induces a mapping $h_{n}^{\star}: 2^{\mathbb{R}} \rightarrow \mathcal{F}_{c}^{1}$ by assigning each set $B \subseteq \mathbb{R}$ its fuzzy relative frequency $h_{n}^{\star}(B)$. In the sequel some main properties of this mapping will be observed - in doing so the following notation will be used for every $\alpha \in(0,1]$ and every $B \in \mathcal{B}(\mathbb{R})$ :

$$
\left[\underline{\mathfrak{h}}_{n, \alpha}(B), \overline{\mathfrak{h}}_{n, \alpha}(B)\right]:=\left[h_{n}^{\star}(B)\right]_{\alpha}
$$

According to equality (2.5) it follows immediately that for fixed $n \in \mathbb{N}$ and $B \subseteq \mathbb{R}$ both $\underline{\mathfrak{h}}_{n, \alpha}(B)=\underline{h}_{n, \alpha}(B)$ and $\overline{\mathfrak{h}}_{n, \alpha}(B)=\bar{h}_{n, \alpha}(B)$ holds for all except at most finitely many $\alpha \in(0,1]$.

Remark: Fix $n \in \mathbb{N}$ and $B \subseteq \mathbb{R}$. Then obviously $\underline{h}_{n, \alpha}(B)$ as a function of $\alpha$ is a monotonically increasing step function in $\alpha \in(0,1]$. The function $\underline{\mathfrak{h}}_{n, \alpha}(B)$, also regarded as a function of $\alpha$, is a left-continuous and monotonically increasing step function which coincides with $\underline{h}_{n, \alpha}(B)$ at least outside the set of all discontinuities of $\underline{\mathfrak{h}}_{n, \alpha}(B)$, which is finite.

In the same manner $\bar{h}_{n, \alpha}(B)$ as a function of $\alpha$ is a monotonically decreasing step function in $\alpha \in(0,1]$. The function $\overline{\mathfrak{h}}_{n, \alpha}(B)$, also regarded as a function of $\alpha$, is a left-continuous and monotonically decreasing step function which coincides with $\bar{h}_{n, \alpha}(B)$ at least outside the set of all discontinuities of $\overline{\mathfrak{h}}_{n, \alpha}(B)$, which is finite too.

The most important properties of $h_{n}^{\star}(\cdot)$ as fuzzy-valued set function are summarized in the next theorem.

Theorem 2.2 Suppose that $x_{1}^{\star}, x_{2}^{\star}, \ldots, x_{n}^{\star}$ is a fuzzy sample of size $n$ and that $B, C$ are arbitrary subsets of $\mathbb{R}$. Furthermore let the fuzzy frequency $h_{n}^{\star}(\cdot)$ and the real-valued set functions $\underline{\mathfrak{h}}_{n, \alpha}(\cdot)$ and $\overrightarrow{\mathfrak{h}}_{n, \alpha}(\cdot)$ be defined according to (2.5) and (2.6), then:

1. $\operatorname{supp}\left(h_{n}^{\star}(B)\right) \subseteq[0,1]$

2. $h_{n}^{\star}(\mathbb{R})=\mathbf{1}_{[1,1]}=1_{\{1\}}, \quad h_{n}^{\star}(\emptyset)=1_{[0,0]}=1_{\{0\}}$

3. $\underline{\mathfrak{h}}_{n, \alpha}(\cdot)$ and $\overline{\mathfrak{h}}_{n, \alpha}(\cdot)$ are monotonic set-functions for every $\alpha \in(0,1]$, i.e. $\underline{\mathfrak{h}}_{n, \alpha}(B) \leq \underline{\mathfrak{h}}_{n, \alpha}(C)$ and $\overline{\mathfrak{h}}_{n, \alpha}(B) \leq \overline{\mathfrak{h}}_{n, \alpha}(C)$ hold whenever $B \subseteq C$.

4. $\underline{\mathfrak{h}}_{n, \alpha}(\cdot)$ is a superadditive and $\overline{\mathfrak{h}}_{n, \alpha}(\cdot)$ is a subadditive set function for every $\alpha \in(0,1]$, i.e. if $B \cap C=\emptyset$, then the following inequalities hold 


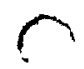

$r$ 
$\curvearrowright$

$r$ 
r

$\bigcap$ 
for every $\alpha \in(0,1]$ :

$$
\begin{aligned}
& \underline{\mathfrak{h}}_{n, \alpha}(B \cup C) \geq \underline{\mathfrak{h}}_{n, \alpha}(B)+\underline{\mathfrak{h}}_{n, \alpha}(C) \text { and } \\
& \overline{\mathfrak{h}}_{n, \alpha}(B \cup C) \leq \overline{\mathfrak{h}}_{n, \alpha}(B)+\overline{\mathfrak{h}}_{n, \alpha}(C)
\end{aligned}
$$

5. $\underline{\mathfrak{h}}_{n, \alpha}\left(B^{c}\right)=1-\overline{\mathfrak{h}}_{n, \alpha}(B)$ and $\overline{\mathfrak{h}}_{n, \alpha}\left(B^{c}\right)=1-\underline{\mathfrak{h}}_{n, \alpha}(B)$ hold for every $\alpha \in(0,1]$.

Proof: Since $h_{n}^{\star}(B)$ is the convex hull of the family $\left(\left[\underline{h}_{n, \alpha}(B), \bar{h}_{n, \alpha}(B)\right]\right)_{\alpha \in(0,1]}$ for every $B \subseteq \mathbb{R}$ by construction, it follows immediately that if $\alpha \in(0,1]$ and if $\left(\alpha_{j}\right)_{j \in \mathbb{N}}$ is a monotonically increasing sequence in $(0, \alpha)$ that converges to $\alpha$, then the following interrelations hold:

$$
\underline{\mathfrak{h}}_{n, \alpha}(B)=\lim _{j \rightarrow \infty} \underline{h}_{n, \alpha_{j}}(B) \text { and } \overline{\mathfrak{h}}_{n, \alpha}(B)=\lim _{j \rightarrow \infty} \bar{h}_{n, \alpha_{j}}(B)
$$

The main advantage of (2.7) over (2.5) concerning the proof is the fact that it holds for every $\alpha \in(0,1]$ and not only $\lambda$-almost everywhere.

Consequently in order to prove the assertions stated in the theorem firstly properties of the family $\left(\left[\underline{h}_{n, \alpha}(B), \bar{h}_{n, \alpha}(B)\right]\right)_{\alpha \in(0,1]}$ are observed and after that, by using (2.7), are transferred to $h_{n}^{\star}(B)$.

Since $\left[\underline{h}_{n, \alpha}(B), \bar{h}_{n, \alpha}(B)\right] \subseteq[0,1]$ is satisfied for every $\alpha \in(0,1]$ it follows immediately that because of $(2.7)\left[h_{n}^{\star}(B)\right]_{\alpha}=\left[\underline{\mathfrak{h}}_{n, \alpha}(B), \overline{\mathfrak{h}}_{n, \alpha}(B)\right] \subseteq[0,1]$ holds for every $\alpha \in(0,1]$, which proves the first assertion of the theorem.

Since obviously $\underline{h}_{n, \alpha}(\mathbb{R})=\bar{h}_{n, \alpha}(\mathbb{R})=1$ and $\underline{h}_{n, \alpha}(\emptyset)=\bar{h}_{n, \alpha}(\emptyset)=0$ is fulfilled for every $\alpha \in(0,1]$, again using (2.7) shows that $\underline{\mathfrak{h}}_{n, \alpha}(\mathbb{R})=\overline{\mathfrak{h}}_{n, \alpha}(\mathbb{R})=1$ and $\underline{\mathfrak{h}}_{n, \alpha}(\emptyset)=\overline{\mathfrak{h}}_{n, \alpha}(\emptyset)=0$, which proves the second assertion of the theorem.

If $B \subseteq C \subseteq \mathbb{R}$ then it follows immediately that

$$
\begin{aligned}
& \left\{i \in\{1, \ldots, n\}:\left[x_{i}^{\star}\right]_{\alpha} \subseteq B\right\} \subseteq\left\{i \in\{1, \ldots, n\}:\left[x_{i}^{\star}\right]_{\alpha} \subseteq C\right\} \text { and } \\
& \left\{i \in\{1, \ldots, n\}:\left[x_{i}^{\star}\right]_{\alpha} \cap B \neq \emptyset\right\} \subseteq\left\{i \in\{1, \ldots, n\}:\left[x_{i}^{\star}\right]_{\alpha} \cap C \neq \emptyset\right\}
\end{aligned}
$$

hold for every $\alpha \in(0,1]$, which in turn implies that $\underline{h}_{n, \alpha}(B) \leq \underline{h}_{n, \alpha}(C)$ and $\bar{h}_{n, \alpha}(B) \leq \bar{h}_{n, \alpha}(C)$ is fulfilled for every $\alpha \in(0,1]$. Applying (2.7) yet another time proves monotonicity of $\underline{\mathfrak{h}}_{n, \alpha}(\cdot)$ and $\overline{\mathfrak{h}}_{n, \alpha}(\cdot)$ for every $\alpha \in(0,1]$, which completes the proof of the third assertion.

If $B, C$ are disjoint then

$$
\begin{aligned}
\underline{h}_{n, \alpha}(B \cup C) & =\frac{1}{n} \#\left\{i \in\{1, \ldots, n\}:\left[x_{i}^{\star}\right]_{\alpha} \subseteq B \cup C\right\} \\
& \geq \frac{1}{n} \#\left\{i \in\{1, \ldots, n\}:\left[x_{i}^{\star}\right]_{\alpha} \subseteq B \text { or }\left[x_{i}^{\star}\right]_{\alpha} \subseteq C\right\} \\
& =\underline{h}_{n, \alpha}(B)+\underline{h}_{n, \alpha}(C)
\end{aligned}
$$


holds for every $\alpha \in(0,1]$, from which again by (2.7) it follows immediately that $\underline{\mathfrak{h}}_{n, \alpha}(B \cup C) \geq \underline{\mathfrak{h}}_{n, \alpha}(B)+\underline{\mathfrak{h}}_{n, \alpha}(C)$ is fulfilled for every $\alpha \in(0,1]$, proving that $\underline{\mathfrak{h}}_{n, \alpha}(\cdot)$ is a superadditive set function for every $\alpha \in(0,1]$.

Furthermore because of

$$
\begin{aligned}
\bar{h}_{n, \alpha}(B \cup C) & =\frac{1}{n} \#\left\{i \in\{1, \ldots, n\}:\left[x_{i}^{\star}\right]_{\alpha} \cap(B \cup C) \neq \emptyset\right\} \\
& =\frac{1}{n} \#\left\{i \in\{1, \ldots, n\}:\left[x_{i}^{\star}\right]_{\alpha} \cap B \neq \emptyset \text { or }\left[x_{i}^{\star}\right]_{\alpha} \cap C \neq \emptyset\right\} \\
& \leq \bar{h}_{n, \alpha}(B)+\bar{h}_{n, \alpha}(C),
\end{aligned}
$$

again (2.7) implies that $\overline{\mathfrak{h}}_{n, \alpha}(B \cup C) \leq \overline{\mathfrak{h}}_{n, \alpha}(B)+\overline{\mathfrak{h}}_{n, \alpha}(C)$ holds for every $\alpha \in(0,1]$, which proves that $\underline{\mathfrak{h}}_{n, \alpha}(\cdot)$ is a subadditive set function for every $\alpha \in(0,1]$. Consequently it remains to prove the last assertion. Using

$$
\left\{i \in\{1, \ldots, n\}:\left[x_{i}^{\star}\right]_{\alpha} \subseteq B^{c}\right\}=\{1, \cdots, n\} \backslash\left\{i \in\{1, \ldots, n\}:\left[x_{i}^{\star}\right]_{\alpha} \cap B \neq \emptyset\right\}
$$

and

$$
\left\{i \in\{1, \ldots, n\}:\left[x_{i}^{\star}\right]_{\alpha} \cap B^{c} \neq \emptyset\right\}=\{1, \cdots, n\} \backslash\left\{i \in\{1, \ldots, n\}:\left[x_{i}^{\star}\right]_{\alpha} \subseteq B\right\}
$$

it follows that $\underline{h}_{n, \alpha}\left(B^{c}\right)=1-\bar{h}_{n, \alpha}(B)$ and $\bar{h}_{n, \alpha}\left(B^{c}\right)=1-\underline{h}_{n, \alpha}(B)$ hold for every $\alpha \in(0,1]$, which by using (2.7) completes the proof of the theorem.

Using the notations (1.20) and (1.21) Theorem 2.2 can be expressed more elegantly as follows:

Theorem 2.3 Suppose that $x_{1}^{\star}, x_{2}^{\star}, \ldots, x_{n}^{\star}$ is a fuzzy sample of size $n$ and that $B, C$ are arbitrary subsets of $\mathbb{R}$. Furthermore let the fuzzy relative frequency $h_{n}^{\star}(\cdot)$ be defined according to (2.5), then:

1. $\operatorname{supp}\left(h_{n}^{\star}(B)\right) \subseteq[0,1]$

2. $h_{n}^{\star}(\mathbb{R})=\mathbf{1}_{[1,1]}=\mathbf{1}_{\{1\}}, \quad h_{n}^{\star}(\emptyset)=\mathbf{1}_{[0,0]}=\mathbf{1}_{\{0\}}$

3. $B \subseteq C \subseteq \mathbb{R} \Longrightarrow h_{n}^{\star}(B) \preceq h_{n}^{\star}(C)$

4. $B \cap C=\emptyset \Longrightarrow h_{n}^{\star}(B \cup C) \subseteq h_{n}^{\star}(B) \oplus h_{n}^{\star}(C)$

5. $h_{n}^{\star}\left(B^{c}\right)=1_{\{1\}} \ominus \mathbb{P}^{\star}(B)$

Having in mind both the interrelation (2.2) and the fact that $h_{n}^{\star}(B)$ is a fuzzy number it is inevitable to consider fuzzy-valued 'probabilities' as generalizations of classical probabilities. Of course the question immediately 
arises, which properties a fuzzy-valued mapping $\mathbb{P}^{\star}: \mathcal{A} \rightarrow \mathcal{F}_{c}^{1}$ on a measure space $(\Omega, \mathcal{A})$ should fulfill in order to be called 'probability', however Theorem 2.3 suggests what properties a meaningful notion at least must satisfy. Such fuzzy-valued mappings will be called fuzzy probability distributions in the sequel, firstly in order to avoid confusion with so-called fuzzy measures treated for instance by Z. Wang and G. Klir (compare [45]), and secondly to indicate that they are also induced by the distribution of fuzzy random variables, which will be shown in Section 4.3.

Before formulating a general definition of fuzzy probability distributions, which will be done in Section 2.4, another natural approach to fuzzy-valued mappings on a system of sets based on a completely different idea will be discussed. In the elementary case, which is presented in subsequent Section 2.2 , this approach goes back to Buckley's fuzzy probabilities ([6]), however filtering out the main idea, a much more general concept of so-called fuzzy probability distributions induced by fuzzy probability densities can be developed. Remarkably this concept fulfills all the properties listed in Theorem 2.3 in a very general setting, which is a strong argument for defining general fuzzy probability distributions in the way it is done in Section 2.4 .

\subsection{Discrete fuzzy probability distributions}

Much of the material presented in this section can be found in Buckley [6], however the presentation is chosen differently in order to point out the main idea that will be used for the mentioned generalization in the next section.

Consider as starting point a soccer match of two different teams $M_{1}$ and $M_{2}$. In this situation three different outcomes are possible:

Team $M_{1}$ wins (event $\{a\}$ ), team $M_{2}$ wins (event $\{b\}$ ) or the match ends in a draw (event $\{c\}$ ).

For none of the three outcomes it is possible to know the exact probabilities, therefore the probabilities are estimated (by using old results), or they are provided by experts.

Because of the unavoidable uncertainties in the assessment of the probabilities, it seems to be more realistic to model these estimations by using fuzzy numbers, or, in the simplest case, by using intervals. Let

$$
\Omega:=\{a, b, c\}, \quad \mathcal{A}:=2^{\Omega}
$$

and suppose that

$$
p(a)=\left[\frac{4}{10}, \frac{5}{10}\right]=: I_{a}
$$




$$
\begin{aligned}
& p(b)=\left[\frac{3}{10}, \frac{6}{10}\right]=: I_{b} \\
& p(c)=\left[\frac{2}{10}, \frac{3}{10}\right]=: I_{c} .
\end{aligned}
$$

The assignment above can be considered to be meaningful and a generalization of punctual probabilities as there exist $x_{1} \in p(a), x_{2} \in p(b), x_{3} \in p(c)$ such that

$$
x_{1}+x_{2}+x_{3}=1 .
$$

Based on the intervals $I_{a}, I_{b}, I_{c}$ 'probabilities' for all subsets of $\Omega$ can be defined in the following way:

$$
\begin{aligned}
& \mathbb{P}(\{a, b\}):=\left\{x_{1}+x_{2}: x_{1} \in I_{a}, x_{2} \in I_{b}, x_{3} \in I_{c}, x_{1}+x_{2}+x_{3}=1\right\} \\
& \mathbb{P}(\{a, c\}):=\left\{x_{1}+x_{3}: x_{1} \in I_{a}, x_{2} \in I_{b}, x_{3} \in I_{c}, x_{1}+x_{2}+x_{3}=1\right\} \\
& \mathbb{P}(\{b, c\}):=\left\{x_{2}+x_{3}: x_{1} \in I_{a}, x_{2} \in I_{b}, x_{3} \in I_{c}, x_{1}+x_{2}+x_{3}=1\right\} \\
& \mathbb{P}(\{a, b, c\}):=\left\{x_{1}+x_{2}+x_{3}: x_{1} \in I_{a}, x_{2} \in I_{b}, x_{3} \in I_{c} x_{1}+x_{2}+x_{3}=1\right\}
\end{aligned}
$$

This yields:

$$
\begin{aligned}
\mathbb{P}(\{a, b\}) & =\left[\frac{7}{10}, \frac{8}{10}\right] \\
\mathbb{P}(\{a, c\}) & =\left[\frac{6}{10}, \frac{7}{10}\right] \\
\mathbb{P}(\{b, c\}) & =\left[\frac{5}{10}, \frac{8}{10}\right] \\
\mathbb{P}(\Omega) & =[1,1] \\
\mathbb{P}(\emptyset) & =[0,0]
\end{aligned}
$$

As in the previous chapter let $\mathcal{F}_{c}^{1}$ denote the set of all fuzzy numbers defined according to Definition 1.1.

Definition 2.4 Let $\Omega=\left\{a_{1}, \cdots, a_{n}\right\}$ be a finite set.

Then a mapping $p^{\star}: \Omega \longrightarrow \mathcal{F}_{c}^{1}$ is called discrete fuzzy probability density on $\Omega$, if for every $i \in\{1, \cdots, n\}$ the fuzzy numbers $p_{i}^{\star}:=p^{\star}\left(a_{i}\right)$ fulfill:

$$
\begin{gathered}
{\left[p_{i}^{\star}\right]_{\alpha} \subseteq[0,1] \quad \forall \alpha \in(0,1] \quad \text { and }} \\
\forall i \in\{1, \cdots, n\}: \exists x_{i} \in\left[p_{i}^{\star}\right]_{1} \text { such that } \sum_{i=1}^{n} x_{i}=1
\end{gathered}
$$


Similar to the above example $\mathbb{P}^{\star}(A)$ is defined on all sets $A \subseteq \Omega$ by

- $\left[\mathbb{P}^{\star}(A)\right]_{\alpha}:=\left\{\sum_{i: a_{i} \in A} x_{i}: x_{i} \in\left[p_{i}^{\star}\right]_{\alpha} \quad \forall i \in\{1, \cdots, n\}, \sum_{i=1}^{n} x_{i}=1\right\}$.

At first sight it may be not clear if for every $A \subseteq \Omega$ the family $\left(\left[\mathbb{P}^{*}(A)\right]_{\alpha}\right)_{\alpha \in(0,1]}$ really is a family of $\alpha$-cuts of a fuzzy number, however the following lemma can be proved.

Lemma 2.5 Suppose $\Omega=\left\{a_{1}, \cdots, a_{n}\right\}$ is a finite set, $A \subseteq \Omega$ an arbitrary subset of $\Omega, p^{\star}: \Omega \longrightarrow \mathcal{F}_{c}^{1}$ is a discrete fuzzy probability density on $\Omega$ and $\left[\mathbb{P}^{\star}(A)\right]_{\alpha}$ is defined according to (2.8), then:

1. $\left[\mathbb{P}^{*}(A)\right]_{\alpha}$ is a compact interval for every $\alpha \in(0,1]$

2. $\left[\mathbb{P}^{\star}(A)\right]_{\alpha}$ is a nested monotonically decreasing family in $\alpha$ with

$$
\bigcap_{\alpha<\beta}\left[\mathbb{P}^{\star}(A)\right]_{\alpha}=\left[\mathbb{P}^{\star}(A)\right]_{\beta} .
$$

Proof: Let $\mathcal{K}_{c}^{n}$ denote the system of all non-empty, compact and convex subsets of $\mathbb{R}^{n}$ and define

$$
\begin{aligned}
\triangle & :=\left\{x=\left(x_{1}, \cdots, x_{n}\right) \in \mathbb{R}^{n}: \quad x_{i} \geq 0 \quad \forall i \text { and } \sum_{i=1}^{n} x_{i}=1\right\} \\
S_{\alpha} & :=\left[p_{1}\right]_{\alpha} \times\left[p_{2}\right]_{\alpha} \times \cdots \times\left[p_{n}\right]_{\alpha} \subseteq \mathbb{R}^{n}, \quad \mathcal{D}_{\alpha}:=S_{\alpha} \cap \triangle, \quad \text { for } \alpha \in(0,1] .
\end{aligned}
$$

Then it follows immediately from the definition that $\mathcal{D}_{\alpha} \neq \emptyset$ holds, which shows that $D_{\alpha} \in \mathcal{K}_{c}^{n}$. The mapping $f: \mathbb{R}^{n} \longrightarrow \mathbb{R}$, defined by

$$
f\left(\left(x_{1}, \cdots, x_{n}\right)\right)=\sum_{i: a_{i} \in A} x_{i},
$$

is linear, continuous and satisfies $f\left(\mathcal{D}_{\alpha}\right)=\left[\mathbb{P}^{\star}(A)\right]_{\alpha}$. As an image of a compact and convex set under a continuous linear mapping, $\left[\mathbb{P}^{\star}(A)\right]_{\alpha}$ is a compact interval, which proves the first part of the lemma.

If $\beta \leq \alpha$ it follows that $\mathcal{D}_{\alpha} \subseteq \mathcal{D}_{\beta}$ and consequently

$$
\left[\mathbb{P}^{\star}(A)\right]_{\alpha} \subseteq\left[\mathbb{P}^{\star}(A)\right]_{\beta} .
$$

The only thing left to show is:

$$
\bigcap_{\alpha<\beta}\left[\mathbb{P}^{\star}(A)\right]_{\alpha}=\left[\mathbb{P}^{\star}(A)\right]_{\beta}
$$


For $x \in \bigcap_{\alpha<\beta}\left[\mathbb{P}^{\star}(A)\right]_{\alpha}$ it follows that for each $\alpha \in(0, \beta)$ there exists $y_{\alpha} \in \mathcal{D}_{\alpha}$ such that $f\left(y_{\alpha}\right)=x$.

Especially for every sequence $\left(\alpha_{n}\right)_{n \in \mathbb{N}}$ with $\alpha_{n} \nearrow \beta$ and $\alpha_{n} \in(0, \beta)$ there exists a sequence $\left(y_{n}\right)_{n \in \mathbb{N}}$ such that $y_{n} \in \mathcal{D}_{\alpha_{n}}$ and $f\left(y_{n}\right)=x$ holds.

Because of the compactness of $\mathcal{D}_{\alpha_{1}}$ there exists a $y \in \mathbb{R}^{n}$ and a convergent subsequence $\left(y_{n_{k}}\right)_{k \in \mathbb{N}}$ of $\left(y_{n}\right)_{n \in \mathbb{N}}$ with

$$
y_{n_{k}} \longrightarrow y \in \bigcap_{l=1}^{\infty} \mathcal{D}_{\alpha_{l}}=\mathcal{D}_{\beta} \quad \text { for } k \rightarrow \infty .
$$

Using the continuity of $f$ it follows that $f(y)=x$ and therefore

$$
x \in\left[\mathbb{P}^{\star}(A)\right]_{\beta} .
$$

The other inclusion is trivial, as for every $\alpha<\beta$ it follows that $\mathcal{D}_{\beta} \subseteq \mathcal{D}_{\alpha}$ and consequently

$$
\left[\mathbb{P}^{\star}(A)\right]_{\beta} \subseteq\left[\mathbb{P}^{\star}(A)\right]_{\alpha}
$$

Lemma 2.5 especially shows that $\mathbb{P}^{\star}(A)$ is a fuzzy number for every $A \subseteq \Omega$. $\mathbb{P}^{\star}(\cdot)$ will be called discrete fuzzy probability distribution induced by the discrete fuzzy probability density $p^{\star}$.

The most important properties of discrete fuzzy probability distributions are summarized in the following theorem, which uses the notions explained at the end of Section 1.3 (compare [6]).

Theorem 2.6 Let $\Omega=\left\{a_{1}, \cdots, a_{n}\right\}$ be a finite set, $\mathbb{P}^{\star}$ a discrete fuzzy probability distribution induced by a discrete fuzzy probability density $p^{\star}$, and $A, B \subseteq \Omega$, then:

1. $\operatorname{supp}\left(\mathbb{P}^{\star}(A)\right) \subseteq[0,1] \quad \forall A \subseteq \Omega$

2. $\mathbb{P}^{\star}(\Omega)=\mathbf{1}_{[1,1]}=\mathbf{1}_{\{1\}}, \quad \mathbb{P}^{\star}(\emptyset)=\mathbf{1}_{[0,0]}=\mathbf{1}_{\{0\}}$

3. $A \subseteq B \Longrightarrow \mathbb{P}^{\star}(A) \preceq \mathbb{P}^{\star}(B) \quad$ (Monotony)

4. $A \cap B=\emptyset \Longrightarrow \mathbb{P}^{\star}(A \cup B) \subseteq \mathbb{P}^{\star}(A) \oplus \mathbb{P}^{\star}(B)$ (Subadditivity)

5. $1 \in\left[\mathbb{P}^{\star}(A) \oplus \mathbb{P}^{\star}\left(A^{c}\right)\right]_{\alpha} \quad \forall \alpha \in(0,1]$

6. $A \cap B \neq \emptyset \Longrightarrow \mathbb{P}^{\star}(A \cup B) \subseteq \mathbb{P}^{\star}(A) \oplus \mathbb{P}^{\star}(B) \ominus \mathbb{P}^{\star}(A \cap B)$

7. $\mathbb{P}^{\star}\left(A^{c}\right)=\mathbf{1}_{\{1\}} \ominus \mathbb{P}^{\star}(A)$

Proof: The above theorem is a special case of Theorem 2.15 and Theorem 2.16 in the next section. 


\subsection{Fuzzy probability distributions induced by fuzzy probability densities}

Let $(\Omega, \mathcal{A}, \mu)$ be an arbitrary measure space and $G: \Omega \longrightarrow \mathcal{K}_{c}^{1}$ an intervalvalued function (also referred as correspondence) on $\Omega$.

A function $g: \Omega \longrightarrow \mathbb{R}$ is called selection of $G$ if $g(t) \in G(t)$ holds for $\mu$-almost every $t \in \Omega$. Note that if $g$ is a selection of $G$ and $h$ is another function, such that $g(t)=h(t)$ holds for $\mu$-almost every $t \in \Omega$, then $h$ is a selection of $G$ too. The set of all measurable selections of $G$ will be denoted by $\operatorname{Sel}(G)$.

Furthermore the correspondence $G$ is called integrably bounded, if there exists an integrable function $h$ such that

$$
|y| \leq h(t) \text { for every } y \in G(t) \text { and every } t \in \Omega \text {. }
$$

If $f^{\star}: \Omega \longrightarrow \mathcal{F}_{c}^{1}$ is a fuzzy-valued function on $\Omega$, then the function $F_{\alpha}$, defined by

$$
F_{\alpha}(t):=\left[f^{\star}(t)\right]_{\alpha}
$$

is an interval-valued function on $\Omega$ for each $\alpha \in(0,1]$.

Moreover for every fuzzy-valued function $f^{\star}: \Omega \longrightarrow \mathcal{F}_{c}^{1}$ the lower and upper $\alpha$-level functions $\underline{f}_{\alpha}(\cdot)$ and $\bar{f}_{\alpha}(\cdot)$ are defined by

$$
\underline{f}_{\alpha}(t):=\min \left(F_{\alpha}(t)\right) \quad \text { and } \quad \bar{f}_{\alpha}(t):=\max \left(F_{\alpha}(t)\right)
$$

for every $t \in \Omega$ and every $\alpha \in(0,1]$. In other words the following identity holds for every $t \in \Omega$ and every $\alpha \in(0,1]$ :

$$
\left[\underline{f}_{\alpha}(t), \bar{f}_{\alpha}(t)\right]=F_{\alpha}(t) \in \mathcal{K}_{c}^{1}
$$

The fuzzy valued function $f^{\star}$ is called (uniformly) integrably bounded, if there exists an integrable function $h$ such that for $\mu$-almost every $t \in \Omega$

$$
|y| \leq h(t) \text { for every } y \in \operatorname{supp}\left(f^{\star}(t)\right) .
$$

Using these notions the following general definition can be made:

Definition 2.7 Let $(\Omega, \mathcal{A}, \mu)$ be an arbitrary measure space.

Then a function $f^{\star}: \Omega \longrightarrow \mathcal{F}_{c}^{1}$ is called fuzzy probability density with respect to the measure $\mu$ on $(\Omega, \mathcal{A})$, if for $F_{\alpha}$ the following conditions are fulfilled:

1. $F_{\alpha}(t) \subseteq[0, \infty) \quad \forall t \in \Omega, \quad \forall \alpha \in(0,1]$

2. $\exists f \in \operatorname{Sel}\left(F_{1}\right)$ such that $\int_{\Omega} f(t) d \mu(t)=1$ 
Definition 2.7 includes and unifies

- discrete fuzzy probability distributions induced by discrete fuzzy probability densities (the measure in this case is the counting measure)

- classical probability densities on a measure space $(\Omega, \mathcal{A}, \mu)$

- classical parametric probability densities (depending continuously on the parameter) with a fuzzy parameter as treated in [6] (compare Example 2.8).

Remark: According to Definition 2.7 a fuzzy probability density $f^{\star}$ doesn't have to fulfill any measurability requirements except the fact that there are some measurable selections.

Fuzzy probability densities can appear as depicted in Figure 2.3 - Figure 2.4 depicts the same fuzzy probability density from a different point of view and Figure 2.5 depicts some $\alpha$-level curves for this example.

Figure 2.3: Sketch of a fuzzy probability density

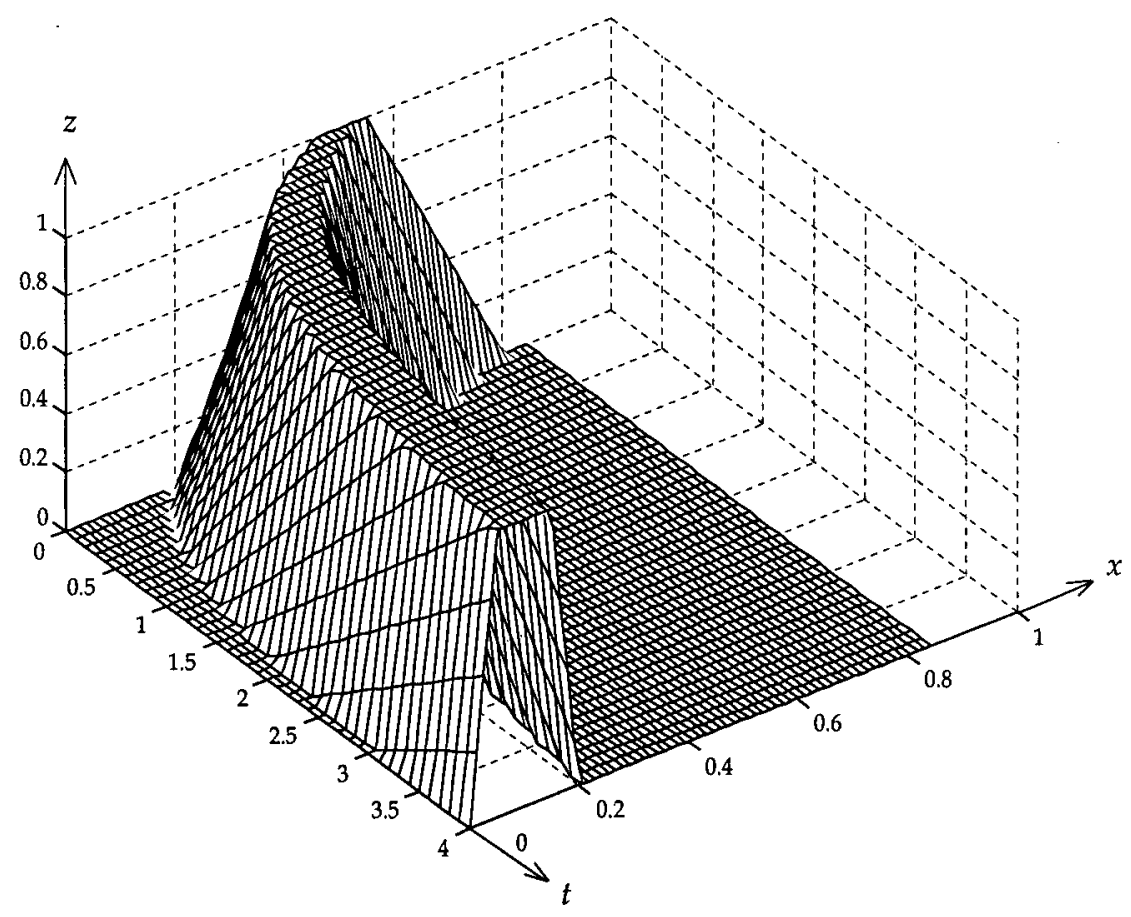


Figure 2.4: Sketch from a different focus

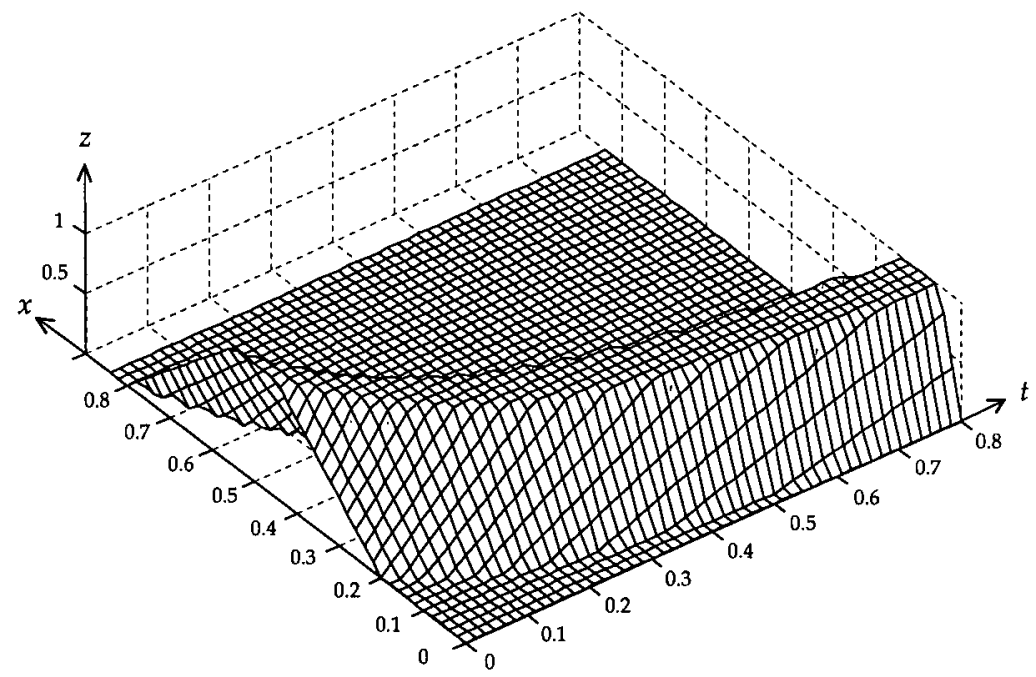

Figure 2.5: Corresponding $\alpha$-level functions

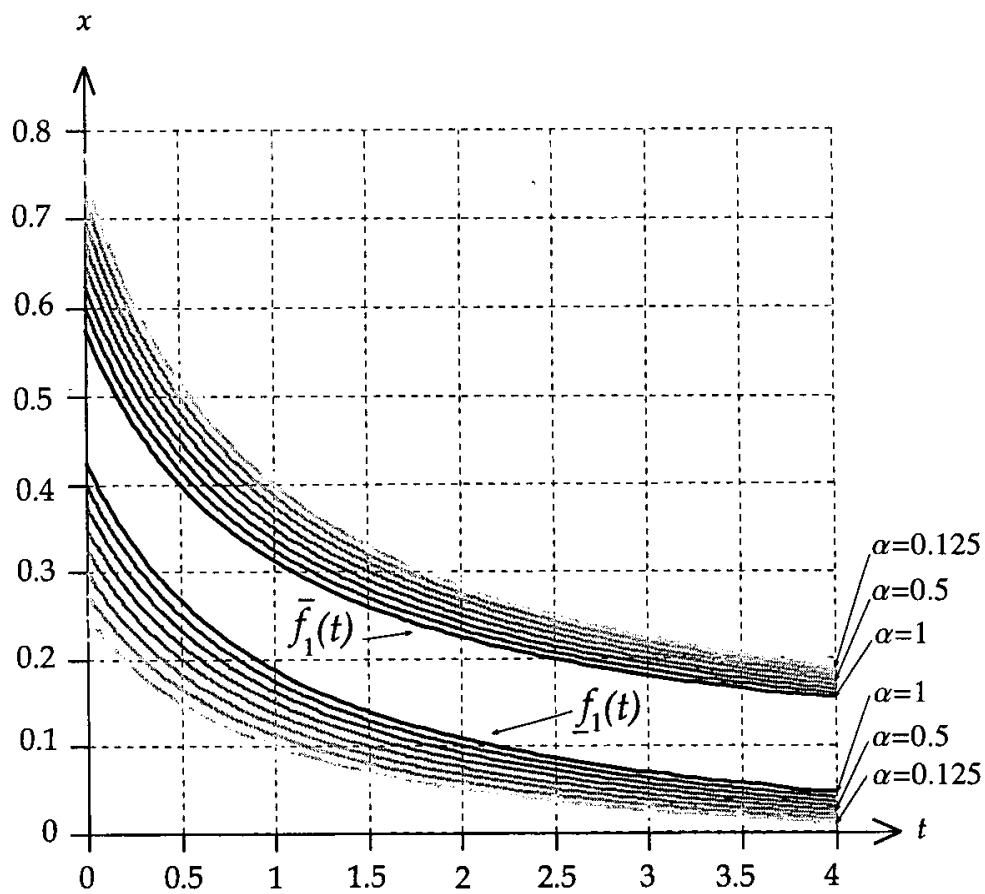


Example 2.8 Consider the well-known exponential density $g_{\eta}$ with parameter $\eta$, defined by $g_{\eta}(t)=\eta e^{-\eta t}$ for every $t \geq 0$ and $\eta>0$. Obviously for every $t \in[0, \infty)$ the function $h_{t}:(0, \infty) \rightarrow(0, \infty)$, defined by

$$
h_{t}(\eta):=g_{\eta}(t)=\eta e^{-\eta t},
$$

is a continuous (in fact even $C^{\infty}$-) function in $\eta \in(0, \infty)$. Therefore if the parameter $\eta$ is no real number but a fuzzy number $\eta^{\star} \in \mathcal{F}_{c}^{1}$ with support in $(0, \infty)$ then applying the Extension Principle (1.19) and Lemma 1.32 shows that $f^{\star}(t)$, defined by

$$
\left(f^{\star}(t)\right)(y):=\left\{\begin{array}{ll}
0 & \text { if } h_{t}^{-1}(\{y\})=\emptyset \\
\sup \left\{\eta^{\star}(x): x \in h_{t}^{-1}(\{y\}\}\right. & \text { otherwise }
\end{array}\right\} \forall y \in \mathbb{R},
$$

is a fuzzy number for every $t \in[0, \infty)$.

Consequently $f^{\star}(\cdot)$ is a fuzzy-valued mapping on $[0, \infty)$. Moreover because of the fact that for every $\eta \in\left[\eta^{\star}\right]_{1}$ the function $g_{\eta}(\cdot)$ is both a measurable selection of $F_{1}(\cdot)$, and a probability density on $\mathbb{R}$ with respect to the Lebesgue measure $\lambda$ it follows that $f^{\star}$ is a fuzzy probability density on the measure space $\left(\mathbb{R}_{0}^{+}, \mathcal{B}\left(\mathbb{R}_{0}^{+}\right), \lambda\right)$ according to Definition 2.7 .

This example will be continued later on for two special cases of $\eta^{\star}$ (compare Example 2.13 and Example 2.14).

Given a fuzzy probability density $f^{\star}$ with respect to the measure $\mu$ on $(\Omega, \mathcal{A})$, a fuzzy probability distribution $\mathbb{P}^{\star}$ on $\mathcal{A}$ can be defined analogous to the discrete case on all sets $A \in \mathcal{A}$ by:

$$
\left[\mathbb{P}^{\star}(A)\right]_{\alpha}:=\left\{\int_{A} f(t) d \mu(t): f \in \operatorname{Sel}\left(F_{\alpha}\right) \text { and } \int_{\Omega} f(t) d \mu(t)=1\right\}
$$

Using the abbreviation

$$
\widetilde{\mathcal{D}}_{\alpha}:=\left\{f \in \operatorname{Sel}\left(F_{\alpha}\right): \int_{\Omega} f(t) d \mu(t)=1\right\},
$$

the above definition reduces to

$$
\left[\mathbb{P}^{\star}(A)\right]_{\alpha}=\left\{\int_{A} f(t) d \mu(t): f \in \widetilde{\mathcal{D}}_{\alpha}\right\} .
$$

Since every function $f \in \widetilde{\mathcal{D}}_{\alpha}$ is integrable by construction it is clear that $\widetilde{\mathcal{D}}_{\alpha} \subseteq \mathcal{L}^{1}(\Omega, \mathcal{A}, \mu)$ for every $\alpha \in(0,1]$, whereby as usual $\mathcal{L}^{1}(\Omega, \mathcal{A}, \mu)$ denotes the set of all integrable (and hence measurable) real-valued functions on $\Omega$ 
(compare for instance [2]).

In the sequel as common let $L^{1}(\Omega, \mathcal{A}, \mu)$ denote the Banach space of all equivalence classes of functions in $\mathcal{L}^{1}(\Omega, \mathcal{A}, \mu)$ modulo equality $\mu$-almost everywhere (compare [2], [13] or [35]). Although in fact every element of $L^{1}(\Omega, \mathcal{A}, \mu)$ is an equivalence class of measurable functions as customary (and since no confusion will arise) $L^{1}(\Omega, \mathcal{A}, \mu)$ will be considered as a space of functions.

If $f \in \mathcal{D}_{\alpha}$ and $g$ is a measurable function such that $f(t)=g(t)$ for $\mu$-almost every $t \in \Omega$, then obviously both $g \in \mathcal{D}_{\alpha}$, and $\int_{A} f(t) d \mu(t)=\int_{A} g(t) d \mu(t)$ follows for every measurable set $A \in \mathcal{A}$.

Because of that, equation (2.14) can be reformulated as follows:

$$
\left[\mathbb{P}^{\star}(A)\right]_{\alpha}=\left\{\int_{A} f(t) d \mu(t): f \in \mathcal{D}_{\alpha}\right\}
$$

whereby

$$
\mathcal{D}_{\alpha}:=\left\{f \in L^{1}(\Omega, \mathcal{A}, \mu): f \in \operatorname{Sel}\left(F_{\alpha}\right) \text { and } \int_{\Omega} f(t) d \mu(t)=1\right\} .
$$

The reason for considering $\mathcal{D}_{\alpha}$ consists in the fact that $\mathcal{D}_{\alpha}$ is a subset of $L^{1}(\Omega, \mathcal{A}, \mu)$ having a compactness property in the weak topology, that will be very useful in the following (note the analogy to the set $\mathcal{D}_{\alpha} \in \mathcal{K}_{c}^{n}$ used in the proof of Lemma 2.5) .

Theorem 2.9 Let $(\Omega, \mathcal{A}, \mu)$ be an arbitrary measure space, $f^{\star}: \Omega \longrightarrow \mathcal{F}_{c}^{1}$ a fuzzy probability density with respect to the measure $\mu$ on $(\Omega, \mathcal{A})$, and $f^{\star}$ integrably bounded.

Then $\mathcal{D}_{\alpha}$ is a weakly compact subset of $L^{1}(\Omega, \mathcal{A}, \mu)$ for every $\alpha \in(0,1]$.

Proof: In order to prove this theorem two famous results from functional analysis, Theorem 6.11 and Theorem 6.12 in the Appendix, will be used (compare [12],[23],[36]).

Since the fuzzy probability density $f^{\star}$ is integrably bounded by assumption, there exists a function $h \in L^{1}(\Omega, \mathcal{A}, \mu)$ (to be precise a representative $h$ of an equivalence class in $L^{1}(\Omega, \mathcal{A}, \mu)$ ), such that

$$
0 \leq|f(t)|=f(t) \leq h(t) \quad \text { for } \mu \text {-almost every } t \in \Omega \quad \forall f \in \mathcal{D}_{\alpha} .
$$

If $\epsilon>0$, there exists a nonnegative measurable simple function $s$ such that $0 \leq s(t) \leq h(t)$ for all $t \in \Omega$ and

$$
\|h-s\|_{1}=\int_{\Omega}|h(t)-s(t)| d \mu(t)<\frac{\epsilon}{2} .
$$


Define $M:=\max \{s(t), t \in \Omega\}<\infty$ and set $\delta=\frac{\epsilon}{2 M}$.

Then for arbitrary $f \in \mathcal{D}_{\alpha}$ and $A \in \mathcal{A}$ with $\mu(A)<\delta$ it follows immediately that

$$
\begin{aligned}
\int_{A}|f(t)| d \mu(t) & =\int_{A} f(t) d \mu(t) \leq \int_{A} h(t) d \mu(t) \\
& =\int_{A}(h(t)-s(t)) d \mu(t)+\int_{A} s(t) d \mu(t) \\
& =\int_{\Omega}|h(t)-s(t)| d \mu(t)+M \mu(A) \\
& <\frac{\epsilon}{2}+\frac{\epsilon}{2}=\epsilon,
\end{aligned}
$$

which proves point 2 of Theorem 6.11 .

In order to prove point 3 set $B=\operatorname{supp}(s)=\{x \in \Omega: s(x) \neq 0\}$.

Then clearly $B$ is measurable, $\mu(B)<\infty$, and for $f \in \mathcal{D}_{\alpha}$ it follows that

$$
\begin{aligned}
\int_{B^{c}}|f(t)| d \mu(t) & =\int_{B^{c}} f(t) d \mu(t) \leq \int_{B^{c}} h(t) d \mu(t) \\
& =\int_{B^{c}}(h(t)-s(t)) d \mu(t)+\int_{B^{c}} s(t) d \mu(t) \\
& \leq \int_{\Omega}|h(t)-s(t)| d \mu(t)+0=\frac{\epsilon}{2}<\epsilon .
\end{aligned}
$$

Having this, according to Theorem 6.11 , the family $\mathcal{D}_{\alpha}$ is weakly precompact in $L^{1}(\Omega, \mathcal{A}, \mu)$.

Because of the fact that $\mathcal{D}_{\alpha}$ is convex, the Theorem of Mazur (Theorem 6.12) shows that the weak closure of $\mathcal{D}_{\alpha}$ is equal to its strong closure. If it can be proved that $\mathcal{D}_{\alpha}$ is closed in the strong topology it will therefore follow that it is weakly closed, which together with the already proved weak precompactness proves the compactness of $\mathcal{D}_{\alpha}$ in the weak topology.

Suppose $\left(f_{n}\right)_{n \in \mathbb{N}}$ is a strongly convergent sequence in $\mathcal{D}_{\alpha}$, with limit $f \in$ $L^{1}(\Omega, \mathcal{A}, \mu)$, then there exists a $\mu$-almost everywhere to $f$ convergent subsequence $\left(f_{n_{k}}\right)_{k \in \mathbb{N}}$. Because of $f_{n_{k}}(t) \in F_{\alpha}(t)$ for $\mu$-almost every $t \in \Omega \forall k \in \mathbb{N}$ it follows that

$$
f(t) \in F_{\alpha}(t) \quad \text { for } \mu \text {-almost every } t \in \Omega \text {. }
$$

As obviously

$$
\int_{\Omega} f(t) d \mu(t)=1
$$

this shows that $f \in \mathcal{D}_{\alpha}$ and proves that $\mathcal{D}_{\alpha}$ is closed in the strong topology. 
Theorem 2.10 Let $(\Omega, \mathcal{A}, \mu)$ be an arbitrary measure space, $A \in \mathcal{A}$ a measurable set, $f^{\star}: \Omega \longrightarrow \mathcal{F}_{c}^{1}$ a fuzzy probability density with respect to the measure $\mu, f^{\star}$ integrably bounded, and $\left[\mathbb{P}^{\star}(A)\right]_{\alpha}$ defined according to (2.14), then:

1. $\left[\mathbb{P}^{\star}(A)\right]_{\alpha}$ is a compact interval for every $\alpha \in(0,1]$

2. $\left[\mathbb{P}^{\star}(A)\right]_{\alpha}$ is a nested family monotonically decreasing in $\alpha$ with

$$
\bigcap_{\alpha<\beta}\left[\mathbb{P}^{\star}(A)\right]_{\alpha}=\left[\mathbb{P}^{\star}(A)\right]_{\beta}
$$

Proof: The mapping $\Phi_{A}$, defined by

$$
\begin{aligned}
\Phi_{A}: L^{1}(\Omega, \mathcal{A}, \mu) & \longrightarrow \mathbb{R} \\
f & \longmapsto \int_{A} f(t) d \mu(t),
\end{aligned}
$$

is a continuous linear functional on $L^{1}(\Omega, \mathcal{A}, \mu)$ and therefore also continuous in the weak topology. Moreover it satisfies

$$
\Phi_{A}\left(\mathcal{D}_{\alpha}\right)=\left[\mathbb{P}^{\star}(A)\right]_{\alpha} \quad \forall \alpha \in(0,1] .
$$

$\Phi_{A}$ preserves compactness and convexity, which proves the compactness and convexity of $\left[\mathbb{P}^{\star}(A)\right]_{\alpha}$. Apart from that $\left[\mathbb{P}^{\star}(A)\right]_{\alpha} \neq \emptyset$ for all $\alpha \in(0,1]$ by definition, which completes the proof of the first part of the theorem.

Since $\mathcal{D}_{\alpha}$ decreases as $\alpha$ increases, $\left[\mathbb{P}^{\star}(A)\right]_{\alpha}$ is decreasing in $\alpha$ too.

It remains to show that

$$
\bigcap_{\alpha<\beta}\left[\mathbb{P}^{\star}(A)\right]_{\alpha}=\left[\mathbb{P}^{\star}(A)\right]_{\beta}
$$

Suppose $x \in[\mathbb{P}(A)]_{\alpha}$ for all $\alpha<\beta$ and $\left(\alpha_{n}\right)_{n \in \mathbb{N}}$ is a strictly increasing sequence of positive real numbers converging to $\beta$.

Then for every $n \in \mathbb{N}$ there exists a function $f_{n} \in \mathcal{D}_{\alpha_{n}}$ such that

$$
\int_{A} f_{n}(t) d \mu(t)=x
$$

Because of $f_{n} \in \mathcal{D}_{\alpha_{1}} \quad \forall n \in \mathbb{N}$ and the fact that $\mathcal{D}_{\alpha}$ is weakly compact for every $\alpha \in(0,1]$, it follows that there exists a function $f \in \mathcal{D}_{\alpha_{1}}$ and a subsequence $\left(f_{n_{k}}\right)_{k \in \mathbb{N}}$ such that

$$
f_{n_{k}} \longrightarrow f \text { weakly for } k \rightarrow \infty .
$$


Since for each $j$ almost all $f_{n_{k}}$ lie in $\mathcal{D}_{\alpha_{j}}$ it follows, using the same argument, that $f \in \mathcal{D}_{\alpha_{j}}$.

Using

$$
\bigcap_{n=1}^{\infty} F_{\alpha_{n}}(t)=F_{\beta}(t)
$$

one sees that $f(t) \in F_{\beta}(t)$ almost everywhere and therefore $f \in \mathcal{D}_{\beta}$. This shows that $x \in\left[\mathbb{P}^{\star}(A)\right]_{\beta}$ and

$$
\bigcap_{\alpha<\beta}\left[\mathbb{P}^{\star}(A)\right]_{\alpha} \subseteq\left[\mathbb{P}^{\star}(A)\right]_{\beta}
$$

The other inclusion is obvious.

In particular Theorem 2.10 shows that, provided the above conditions hold, $\mathbb{P}^{\star}(A)$, defined by

$$
\left[\mathbb{P}^{\star}(A)\right]_{\alpha}=\left\{\int_{A} f(t) d \mu(t): f \in \mathcal{D}_{\alpha}\right\},
$$

is a fuzzy number (defined via its $\alpha$-cuts).

Definition 2.11 Let $(\Omega, \mathcal{A}, \mu)$ be an arbitrary measure space, $A \in \mathcal{A}$ a measurable set, $f^{\star}: \Omega \longrightarrow \mathcal{F}_{c}^{1}$ a fuzzy probability density with respect to the measure $\mu$, and $f^{\star}$ integrably bounded, then the fuzzy number $\mathbb{P}^{\star}(A)$ defined according to (2.14) is called probability of the event $A$ and $\mathbb{P}^{\star}$ is called fuzzy probability distribution on $(\Omega, \mathcal{A})$.

At first sight the definition (2.14) of the fuzzy probability distribution $\mathbb{P}^{\star}$ induced by a fuzzy probability density $f^{\star}$ may seem difficult to compute in practise - nonetheless in many situation the computation of the fuzzy number $\mathbb{P}^{\star}(A)$ can be reduced to the calculation of integrals of the corresponding $\alpha$ level functions (compare [41]). In order to formulate a precise theorem the following abbreviations will be used for every $\alpha \in(0,1]$ and every $A \in \mathcal{A}$ :

$$
\underline{p}_{\alpha}(A):=\min \left[\mathbb{P}^{\star}(A)\right]_{\alpha}, \bar{p}_{\alpha}(A):=\max \left[\mathbb{P}^{\star}(A)\right]_{\alpha}
$$

Theorem 2.12 Let $(\Omega, \mathcal{A}, \mu)$ be an arbitrary measure space, $A \in \mathcal{A}$ a measurable set, $f^{\star}: \Omega \longrightarrow \mathcal{F}_{c}^{1}$ a fuzzy probability density with respect to the measure $\mu$, and $f^{\star}$ integrably bounded. Furthermore suppose that the corresponding $\alpha$-level functions $\underline{f}_{\alpha}$ and $\bar{f}_{\alpha}$ are measurable for every $\alpha \in(0,1]$. Then for every $\alpha \in(0,1] \underline{p}_{\alpha}(A)$ and $\bar{p}_{\alpha}(A)$ can be calculated as follows:

$\underline{p}_{\alpha}(A)= \begin{cases}\int_{A} \underline{f}_{\alpha}(t) d \mu(t) & \text { if } \int_{A} \underline{f}_{\alpha}(t) d \mu(t)+\int_{A^{c}} \bar{f}_{\alpha}(t) d \mu(t) \geq 1 \\ 1-\int_{A^{c}} \bar{f}_{\alpha}(t) d \mu(t) & \text { otherwise }\end{cases}$ 
$\bar{p}_{\alpha}(A)= \begin{cases}\int_{A} \bar{f}_{\alpha}(t) d \mu(t) & \text { if } \int_{A} \bar{f}_{\alpha}(t) d \mu(t)+\int_{A^{c}} \underline{f}_{\alpha}(t) d \mu(t) \leq 1 \\ 1-\int_{A^{c}} \underline{f}_{\alpha}(t) d \mu(t) & \text { otherwise }\end{cases}$

Proof: It follows immediately from Definition 2.7, that under the assumptions of Theorem 2.12 both

$$
\int_{\Omega} \underline{f}_{\alpha}(t) d \mu(t) \leq 1 \quad \text { and } \quad \int_{\Omega} \vec{f}_{\alpha}(t) d \mu(t) \geq 1
$$

hold for every $\alpha \in(0,1]$. Fix an arbitrary $\alpha \in(0,1]$.

If $\int_{A} \underline{f}_{\alpha}(t) d \mu(t)+\int_{A^{\mathrm{c}}} \bar{f}_{\alpha}(t) d \mu(t) \geq 1$, then set $\delta:=1-\int_{A} \underline{f}_{\alpha}(t) d \mu(t) \geq 0$. It follows immediately that on the one hand $\int_{A^{c}} \bar{f}_{\alpha}(t) d \mu(t) \geq \delta$, and on the other hand $\int_{A^{c}} \underline{f}_{\alpha}(t) d \mu(t) \leq \delta$ holds for every $\alpha \in(0,1]$. Consequently there exists a unique real number $\theta \in[0,1]$, such that

$$
\theta \int_{A^{c}} \underline{f}_{\alpha}(t) d \mu(t)+(1-\theta) \int_{A^{c}} \bar{f}_{\alpha}(t) d \mu(t)=\delta .
$$

Define a new function $g$ as follows

$$
g(t):=\left\{\begin{array}{ll}
\underline{f}_{\alpha}(t) & \text { if } t \in A \\
\theta \underline{f}_{\alpha}(t)+(1-\theta) \bar{f}_{\alpha}(t) & \text { otherwise }
\end{array}\right\} \forall t \in \Omega .
$$

Obviously $g$ is a measurable selection of $F_{\alpha}$ and $\int_{\Omega} g(t) d \mu(t)=1$, so $g \in \mathcal{D}_{\alpha}$. Furthermore obviously $\underline{p}_{\alpha}(A)=\int_{A} g(t) d \mu(t)$ holds.

If $\int_{A} \underline{f}_{\alpha}(t) d \mu(t)+\int_{A^{c}} \bar{f}_{\alpha}(t) d \mu(t)<1$ is fulfilled, then set $\delta:=1-\int_{A^{c}} \bar{f}_{\alpha}(t) d \mu(t)$. This time it follows immediately that on the one hand $\int_{A} \underline{f}_{\alpha}(t) d \mu(t) \leq \delta$, and on the other hand $\int_{A} \bar{f}_{\alpha}(t) d \mu(t) \geq \delta$ holds for every $\alpha \in(0,1]$. Consequently there exists a unique real number $\theta \in[0,1]$ such that

$$
\theta \int_{A} \underline{f}_{\alpha}(t) d \mu(t)+(1-\theta) \int_{A} \bar{f}_{\alpha}(t) d \mu(t)=\delta
$$

Again define a new function $g$ as follows

$$
g(t):=\left\{\begin{array}{ll}
\bar{f}_{\alpha}(t) & \text { if } t \in A^{c} \\
\theta \underline{f}_{\alpha}(t)+(1-\theta) \bar{f}_{\alpha}(t) & \text { otherwise }
\end{array}\right\} \forall t \in \Omega
$$

Obviously $g$ is a measurable selection of $F_{\alpha}$ and $\int_{\Omega} g(t) d \mu(t)=1$, so $g \in \mathcal{D}_{\alpha}$. Furthermore obviously $\underline{p}_{\alpha}(A)=\int_{A} g(t) d \mu(t)$ holds.

This completes the proof of the formula for the calculation of $\underline{p}_{\alpha}(A)$ since $\alpha$ was arbitrary. The formula for the calculation of $\bar{p}_{\alpha}(A)$ can be proved completely in the same manner. 
In the following the concrete calculation is demonstrated for the fuzzy probability density $f^{\star}$ in Example 2.8 with two different cases of $\eta^{\star}$ (compare Figure 2.6): First of all for $\eta^{\star}$ being an interval-type fuzzy number $\iota^{\star}=\mathbf{1}_{[1,2]}$ and after that for $\eta^{\star}$ being a triangular fuzzy number $\tau^{\star}$ defined by

$$
\tau^{\star}(x):=\left\{\begin{array}{ll}
2 x-2 & \text { if } x \in\left[1, \frac{3}{2}\right] \\
-2 x+4 & \text { if } x \in\left(\frac{3}{2}, 2\right] \\
0 & \text { otherwise }
\end{array}\right\} \forall x \in \mathbb{R} .
$$

Figure 2.6: Parameters $\tau^{\star}$ and $\iota^{\star}$ for the exponential density

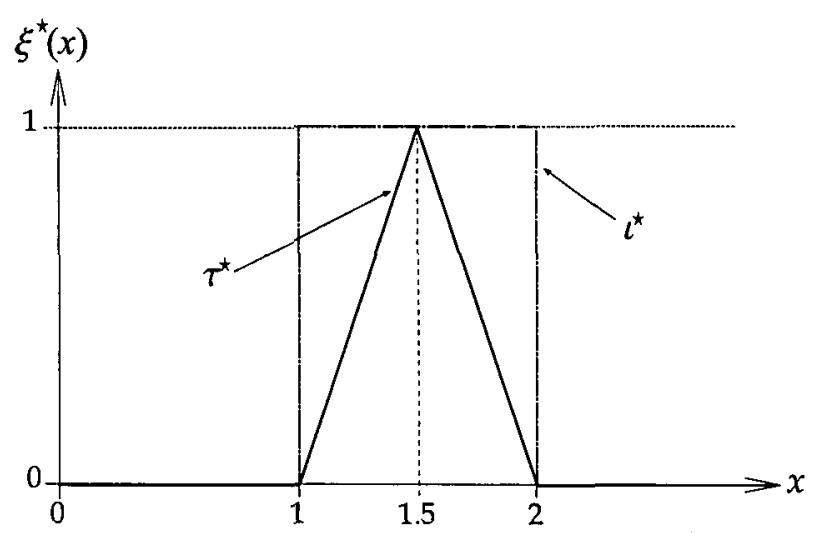

Example 2.13 Exponential density with fuzzy parameter $\eta^{\star}=\mathbf{1}_{[1,2]}$ :

In this case it is very easy to calculate the lower and upper $\alpha$-level functions $\underline{f}_{\alpha}$ and $\bar{f}_{\alpha}$ of $f^{\star}$ explicitly by elementary calculus, since according to the Extension Principle (1.19), Lemma 1.32 and Example 2.8, the following equalities hold for every $t \in[0, \infty)$ :

$$
\begin{aligned}
& \underline{f}_{1}(t)=\min \left\{\eta e^{-\eta t}: \eta \in[1,2]\right\} \\
& \bar{f}_{1}(t)=\max \left\{\eta e^{-\eta t}: \eta \in[1,2]\right\}
\end{aligned}
$$

This minimization and maximization can easily be accomplished by simply computing local extrema and the corresponding boundary values, and yields the following result:

$$
\underline{f}_{1}(t)=\left\{\begin{array}{ll}
e^{-t} & \text { if } t \in[0, \ln 2] \\
2 e^{-2 t} & \text { if } t \in(\ln 2, \infty)
\end{array}\right\} \forall t \in[0, \infty)
$$




$$
\bar{f}_{1}(t)=\left\{\begin{array}{ll}
2 e^{-2 t} & \text { if } t \in[0,1 / 2] \\
\frac{1}{t e} & \text { if } t \in(1 / 2,1] \\
e^{-t} & \text { if } t \in(1, \infty)
\end{array}\right\} \forall t \in[0, \infty)
$$

Since $\eta^{\star}$ is an interval-type fuzzy number all $\alpha$-cuts are identical and it suffices to compute the boundaries of the 1 -cut. The lower and upper $\alpha$-level functions

Figure 2.7: 1 -cut of an exponential density with fuzzy parameter $\eta^{\star}=\mathbf{1}_{[1,2]}$

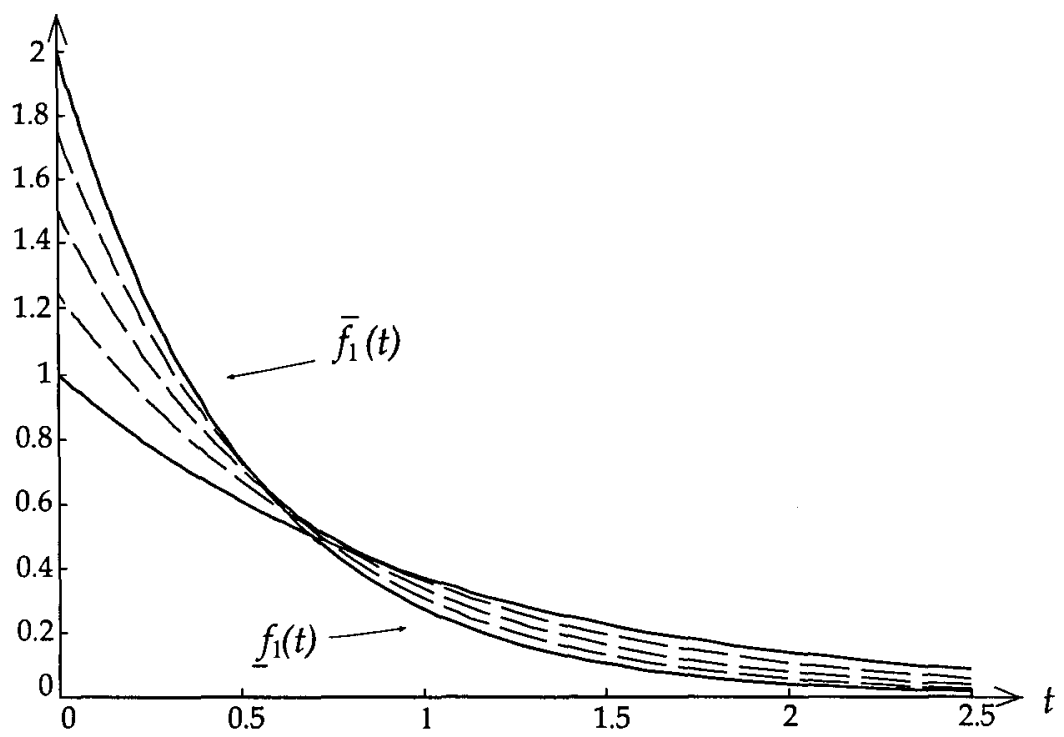

as well as classical densities $g_{\eta}$, where $\eta \in\{1,1.25,1.5,1.75,2\}$, are depicted in Figure 2.7. Obviously the lower and upper $\alpha$-level functions are continuous and bounded, so if one wishes for instance to calculate $\mathbb{P}^{\star}(A)$ for the set $A=[0,2]$, then according to Theorem 2.12 this can be done by simply checking the conditions (2.17) and (2.18) for the set $A$, which gives

$$
\begin{aligned}
& \int_{[0,2]} \underline{f}_{1}(t) d \lambda(t)+\int_{(2, \infty)} \bar{f}_{1}(t) d \lambda(t)<1 \\
& \int_{[0,2]} \bar{f}_{1}(t) d \lambda(t)+\int_{(2, \infty)} \underline{f}_{1}(t) d \lambda(t)>1 .
\end{aligned}
$$

Consequently $\underline{p}_{1}([0,2])$ and $\bar{p}_{1}([0,2])$ can be calculated as follows:

$$
\underline{p}_{1}([0,2])=1-\int_{(2, \infty)} \bar{f}_{1}(t) d \lambda(t)=1-\int_{2}^{\infty} e^{-t} d \lambda(t)
$$




$$
\begin{aligned}
& =1-e^{-2} \doteq 0.865 \\
\bar{p}_{1}([0,2]) & =1-\int_{(2, \infty)} \underline{f}_{1}(t) d \lambda(t)=1-2 \int_{2}^{\infty} e^{-2 t} d \lambda(t) \\
& =1-e^{-4} \doteq 0.982
\end{aligned}
$$

Altogether, this implies that for $\eta^{\star}=\mathbf{1}_{[1,2]}$ the fuzzy number $\mathbb{P}^{\star}([0,2])$ is given by

$$
\mathbb{P}^{\star}([0,2])=\mathbf{1}_{\left[1-e^{-2}, 1-e^{-4}\right]} \text {. }
$$

In completely the same manner one can compute $\mathbb{P}^{\star}\left(A^{\prime}\right)$ for the set $A^{\prime}=[0,1 / 2]$ : First, the conditions (2.17) and (2.18) are checked, which gives

$$
\begin{aligned}
& \int_{[0,1 / 2]} \underline{f}_{1}(t) d \lambda(t)+\int_{(1 / 2, \infty)} \bar{f}_{1}(t) d \lambda(t)>1 \\
& \int_{[0,1 / 2]} \bar{f}_{1}(t) d \lambda(t)+\int_{(1 / 2, \infty)} \underline{f}_{1}(t) d \lambda(t)<1,
\end{aligned}
$$

and then $\underline{p}_{1}([0,1 / 2])$ and $\bar{p}_{1}([0,1 / 2])$ are calculated by:

$$
\begin{aligned}
\underline{p}_{1}([0,1 / 2]) & =\int_{[0,1 / 2)} \underline{f}_{1}(t) d \lambda(t)=\int_{0}^{1 / 2} e^{-t} d \lambda(t) \\
& =1-e^{-1 / 2} \doteq 0.394 \\
\bar{p}_{1}([0,1 / 2]) & =\int_{[0,1 / 2)} \bar{f}_{1}(t) d \lambda(t)=2 \int_{2}^{\infty} e^{-2 t} d \lambda(t) \\
& =1-e^{-1} \doteq 0.632
\end{aligned}
$$

This in turn shows that for $\eta^{\star}=\mathbf{1}_{[1,2]}$ the fuzzy number $\mathbb{P}^{\star}([0,1 / 2])$ is given by

$$
\mathbb{P}^{\star}([0,1 / 2])=\mathbf{1}_{\left[1-e^{-1 / 2}, 1-e^{-1}\right]} .
$$

Both fuzzy numbers $\mathbb{P}^{\star}([0,2])$ and $\mathbb{P}^{\star}([0,1 / 2])$ are depicted in Figure 2.8 .

Example 2.14 Exponential density with fuzzy parameter $\eta^{\star}=\tau^{\star}$ defined according to (2.19):

First of all it follows immediately that the $\alpha$-cuts of $\tau^{\star}$ are given by

$$
\left[\tau^{\star}\right]_{\alpha}=\left[1+\frac{\alpha}{2}, 2-\frac{\alpha}{2}\right]
$$


Figure 2.8: $\mathbb{P}^{\star}([0,1 / 2])$ and $\mathbb{P}^{\star}([0,2])$ for the exponential density with fuzzy parameter $\eta^{\star}=\mathbf{1}_{[1,2]}$

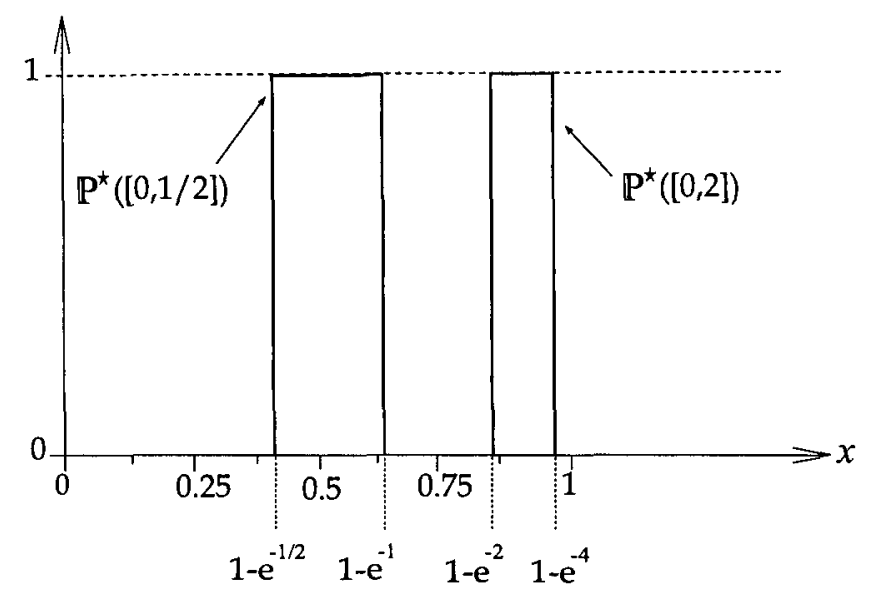

for every $\alpha \in(0,1]$.

For this case too it is possible to calculate the lower and upper boundary functions $f_{\alpha}$ and $\bar{f}_{\alpha}$ explicitly by elementary calculus. According to the Extension Principle (1.19), Lemma 1.32 and Example 2.8 the following equalities hold for every $t \in[0, \infty)$ and every $\alpha \in(0,1]$ :

$$
\begin{aligned}
& \underline{f}_{\alpha}(t)=\min \left\{\eta e^{-\eta t}: \eta \in\left[\tau^{\star}\right]_{\alpha}\right\} \\
& \bar{f}_{\alpha}(t)=\max \left\{\eta e^{-\eta t}: \eta \in\left[\tau^{\star}\right]_{\alpha}\right\}
\end{aligned}
$$

For $\alpha=1$ minimization and maximization are trivial since the 1 -cut $\left[\tau^{\star}\right]_{1}$ of $\tau^{\star}$ is a single point $\left[\tau^{\star}\right]_{1}=\{3 / 2\}$, which immediately implies that

$$
\underline{f}_{1}(t)=\bar{f}_{1}(t)=\frac{3}{2} e^{\frac{3}{2} t} \quad \text { for every } t \in[0, \infty) .
$$

For $\alpha \in(0,1)$ again minimization and maximization can be accomplished by simply computing local extrema in $\eta$ for fixed $t$ (the only local extremum is a local maximum for certain values of $t$ ) and comparing them with the corresponding boundary values. This yields the following result for every $\alpha \in(0,1)$ :

$$
\underline{f}_{\alpha}(t)=\left\{\begin{array}{ll}
\left(1+\frac{\alpha}{2}\right) e^{-t-\frac{\alpha t}{2}} & \text { if } t \in\left[0, \frac{1}{1-\alpha} \ln \left(\frac{4-\alpha}{2+\alpha}\right)\right] \\
\left(2-\frac{\alpha}{2}\right) e^{-2 t+\frac{\alpha t}{2}} & \text { if } t \in\left(\frac{1}{1-\alpha} \ln \left(\frac{4-\alpha}{2+\alpha}\right), \infty\right)
\end{array}\right\} \forall t \in[0, \infty)
$$




$$
\bar{f}_{\alpha}(t)=\left\{\begin{array}{cl}
\left(2-\frac{\alpha}{2}\right) e^{-2 t+\frac{\alpha t}{2}} & \text { if } t \in\left[0, \frac{2}{4-\alpha}\right] \\
\frac{1}{t e} & \text { if } t \in\left(\frac{2}{4-\alpha}, \frac{2}{2+\alpha}\right] \\
\left(1+\frac{\alpha}{2}\right) e^{-t-\frac{\alpha t}{2}} & \text { if } t \in\left(\frac{2}{2+\alpha}, \infty\right)
\end{array}\right\} \forall t \in[0, \infty)
$$

Obviously for every $\alpha \in(0,1)$ both $\underline{f}_{\alpha}(\cdot)$ and $\bar{f}_{\alpha}(\cdot)$ are continuous bounded functions. Consequently the calculation of $\mathbb{P}^{\star}([0,2])$ according to Theorem 2.12 can be done by simply checking the conditions (2.17) and (2.18) for the set $A=[0,2]$ for every $\alpha \in(0,1)$.

Since $\underline{f}_{1}(t)=\bar{f}_{1}(t)=\frac{3}{2} e^{\frac{3}{2} t}$ holds for every $t \in[0, \infty)$ it follows immediately that

$$
\begin{aligned}
{\left[\mathbb{P}^{\star}([0,2])\right]_{1} } & =\left[\underline{p}_{1}([0,2]), \bar{p}_{1}([0,2])\right] \\
& =\left[\int_{[0,2]} \frac{3}{2} e^{-\frac{3}{2} t} d \lambda(t), \int_{[0,2]} \frac{3}{2} e^{-\frac{3}{2} t} d \lambda(t)\right] \\
& =\left[1-e^{-3}, 1-e^{-3}\right]=\left\{1-e^{-3}\right\} .
\end{aligned}
$$

Apart from that it can be verified easily by using standard mathematical software like Maple or Matlab (or with more effort analytically) that

$$
\int_{[0,2]} \underline{f}_{\alpha}(t) d \lambda(t)+\int_{(2, \infty)} \bar{f}_{\alpha}(t) d \lambda(t)<1
$$

holds for very $\alpha \in(0,1)$, which according to Theorem 2.12 implies that

$$
\begin{aligned}
\underline{p}_{\alpha}([0,2]) & =1-\int_{(2, \infty)} \bar{f}_{\alpha}(t) d \lambda(t)=1-\int_{(2, \infty)}\left(1+\frac{\alpha}{2}\right) e^{-t-\frac{\alpha t}{2}} d \lambda(t) \\
& =1-e^{-2-\alpha} .
\end{aligned}
$$

Moreover again with standard software or analytically it can be verified that for every $\alpha \in(0,1)$

$$
\int_{[0,2]} \bar{f}_{\alpha}(t) d \lambda(t)+\int_{(2, \infty)} \underline{f}_{\alpha}(t) d \lambda(t)>1
$$

which in turn shows that

$$
\begin{aligned}
\bar{p}_{\alpha}([0,2]) & =1-\int_{(2, \infty)} \underline{f}_{\alpha}(t) d \lambda(t)=1-\int_{(2, \infty)}\left(2-\frac{\alpha}{2}\right) e^{-2 t+\frac{\alpha t}{2}} d \lambda(t) \\
& =1-e^{-4+\alpha} .
\end{aligned}
$$

Consequently the $\alpha$-cuts of $\mathbb{P}^{\star}([0,2]) \in \mathcal{F}_{c}^{1}$ are given by

$$
\left[\mathbb{P}^{\star}([0,2])\right]_{\alpha}=\left[\underline{p}_{\alpha}([0,2]), \bar{p}_{\alpha}([0,2])\right]=\left[1-e^{-2-\alpha}, 1-e^{-4+\alpha}\right] .
$$


Having that the characterizing function of the fuzzy number $\mathbb{P}^{\star}([0,2])$ can be expressed explicitly as follows (compare Figure 2.9):

$$
\left(\mathbb{P}^{\star}([0,2])\right)(x)=\left\{\begin{array}{ll}
0 & \text { if } x \in\left(-\infty, 1-e^{-2}\right) \\
4+\ln (1-x) & \text { if } x \in\left[1-e^{-2}, 1-e^{-3}\right) \\
-2-\ln (1-x) & \text { if } x \in\left[1-e^{-3}, 1-e^{-4}\right] \\
0 & \text { if } x \in\left(1-e^{-4}, \infty\right)
\end{array}\right\} \forall x \in \mathbb{R}
$$

In order to calculate $\mathbb{P}^{\star}\left(\left[0, \frac{1}{2}\right]\right) \in \mathcal{F}_{c}^{1}$ first of all notice that because of the fact that $\underline{f}_{1}(t)=\bar{f}_{1}(t)=\frac{3}{2} e^{\frac{3}{2} t}$ holds for every $t \in[0, \infty)$ it follows immediately that

$$
\begin{aligned}
{\left[\mathbb{P}^{\star}([0,1 / 2])\right]_{1} } & =\left[\underline{p}_{1}([0,1 / 2]), \bar{p}_{1}([0,1 / 2])\right] \\
& =\left[\int_{[0,1 / 2]} \frac{3}{2} e^{-\frac{3}{2} t} d \lambda(t), \int_{[0,1 / 2]} \frac{3}{2} e^{-\frac{3}{2} t} d \lambda(t)\right] \\
& =\left[1-e^{-3 / 4}, 1-e^{-3 / 4}\right]=\left\{1-e^{-3 / 4}\right\}
\end{aligned}
$$

Apart from that in this case it can be shown that

$$
\int_{[0,1 / 2]} \underline{f}_{\alpha}(t) d \lambda(t)+\int_{(1 / 2, \infty)} \bar{f}_{\alpha}(t) d \lambda(t)>1
$$

holds for very $\alpha \in(0,1)$, which according to Theorem 2.12 implies that

$$
\begin{aligned}
\underline{p}_{\alpha}([0,1 / 2]) & =\int_{[0,1 / 2]} \underline{f}_{\alpha}(t) d \lambda(t)=\int_{[0,1 / 2]}\left(1+\frac{\alpha}{2}\right) e^{-t-\frac{\alpha t}{2}} d \lambda(t) \\
& =1-e^{-\frac{1}{2}-\frac{\alpha}{4}}
\end{aligned}
$$

Moreover it can be verified that for every $\alpha \in(0,1)$

$$
\int_{[0,1 / 2]} \bar{f}_{\alpha}(t) d \lambda(t)+\int_{(1 / 2, \infty)} \underline{f}_{\alpha}(t) d \lambda(t)<1,
$$

which in turn shows that

$$
\begin{aligned}
\bar{p}_{\alpha}([0,1 / 2]) & =\int_{[0,1 / 2]} \bar{f}_{\alpha}(t) d \lambda(t)=\int_{[0,1 / 2]}\left(2-\frac{\alpha}{2}\right) e^{-2 t+\frac{\alpha t}{2}} d \lambda(t) \\
& =1-e^{-1+\frac{\alpha}{4}}
\end{aligned}
$$

Consequently the $\alpha$-cuts of $\mathbb{P}^{\star}([0,1 / 2]) \in \mathcal{F}_{c}^{1}$ are given by

$$
\left[\mathbb{P}^{\star}([0,1 / 2])\right]_{\alpha}=\left[\underline{p}_{\alpha}([0,1 / 2]), \bar{p}_{\alpha}([0,1 / 2])\right]=\left[1-e^{-\frac{1}{2}-\frac{\alpha}{4}}, 1-e^{-1+\frac{\alpha}{4}}\right] .
$$


Having that the characterizing function of the fuzzy number $\mathbb{P}^{\star}([0,1 / 2])$ can be expressed explicitly as follows (compare Figure 2.9):

$$
\left(\mathbb{P}^{\star}([0,1 / 2])\right)(x)=\left\{\begin{array}{ll}
0 & \text { if } x \in\left(-\infty, 1-e^{-1 / 2}\right) \\
-2-4 \ln (1-x) & \text { if } x \in\left[1-e^{-1 / 2}, 1-e^{-3 / 4}\right) \\
4+\ln (1-x) & \text { if } x \in\left[1-e^{-3 / 4}, 1-e^{-1}\right] \\
0 & \text { if } x \in\left(1-e^{-1}, \infty\right)
\end{array}\right\} \forall x \in \mathbb{R}
$$

In Figure $2.9 \mathbb{P}^{\star}([0,1 / 2])$ and $\mathbb{P}^{\star}([0,2])$ for the case of $\eta^{\star}=\tau^{\star}$ are depicted by solid lines - furthermore for comparison the corresponding results for the case $\eta^{\star}=\mathbf{1}_{[1,2]}$ (Example 2.13) are depicted by dotted lines.

Figure 2.9: $\mathbb{P}^{\star}([0,1 / 2])$ and $\mathbb{P}^{\star}([0,2])$ for the exponential density with fuzzy parameter $\eta^{\star}=\tau^{\star}$

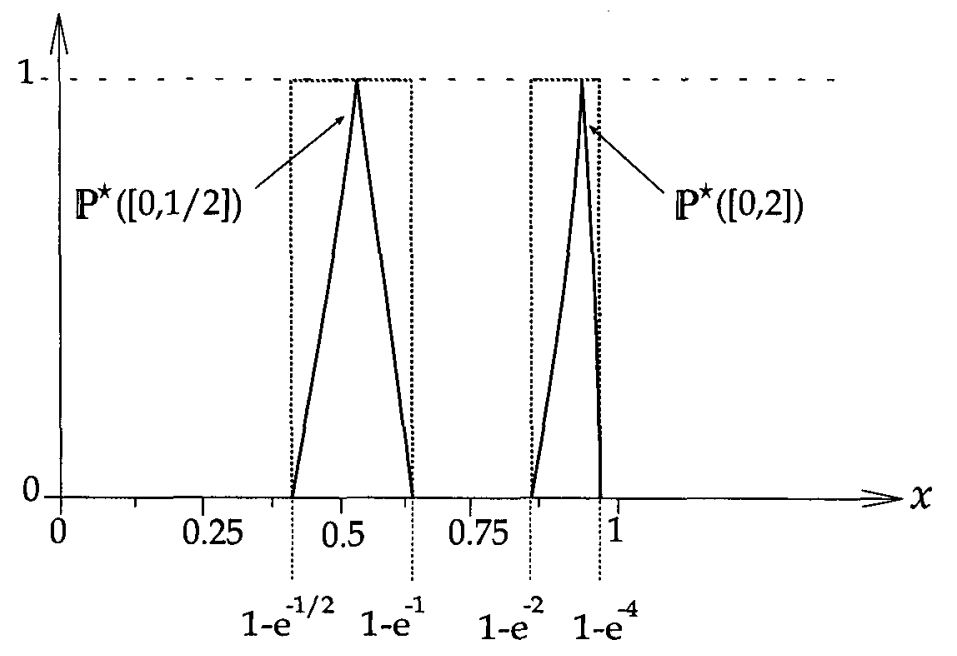

After these examples the main properties of $\mathbb{P}^{\star}$ are analyzed - it will be shown that completely analogous to the discrete case $\mathbb{P}^{\star}$ has the following properties:

Theorem 2.15 Let $(\Omega, \mathcal{A}, \mu)$ be an arbitrary measure space, $f^{\star}: \Omega \longrightarrow \mathcal{F}_{c}^{1} a$ fuzzy probability density with respect to the measure $\mu, f^{\star}$ integrably bounded, $A, B \in \mathcal{A}$ measurable sets, and $\mathbb{P}^{\star}$ defined according to (2.14), then

1. $\operatorname{supp}\left(\mathbb{P}^{\star}(A)\right) \subseteq[0,1] \quad \forall A \in \mathcal{A}$

2. $\mathbb{P}^{\star}(\Omega)=\mathbf{1}_{[1,1]}=\mathbf{1}_{\{1\}}, \quad \mathbb{P}^{\star}(\emptyset)=\mathbf{1}_{[0,0]}=\mathbf{1}_{\{0\}}$ 
3. $A \subseteq B \Longrightarrow \mathbb{P}^{\star}(A) \preceq \mathbb{P}^{\star}(B) \quad$ (Monotony)

4. $A \cap B=\emptyset \Longrightarrow \mathbb{P}^{\star}(A \cup B) \subseteq \mathbb{P}^{\star}(A) \oplus \mathbb{P}^{\star}(B)$ (Subadditivity)

5. $1 \in\left[\mathbb{P}^{\star}(A) \oplus \mathbb{P}^{\star}\left(A^{c}\right)\right]_{\alpha} \quad \forall \alpha \in(0,1]$

6. $A \cap B \neq \emptyset \Longrightarrow \mathbb{P}^{\star}(A \cup B) \subseteq \mathbb{P}^{\star}(A) \oplus \mathbb{P}^{\star}(B) \ominus \mathbb{P}^{\star}(A \cap B)$

Proof: Point one and two are clear by definition.

The third point immediately follows from the fact that for $A \subseteq B$

$$
\int_{A} f(t) d \mu(t) \leq \int_{B} f(t) d \mu(t) \quad \forall f \in \mathcal{D}_{\alpha}
$$

Suppose now that $A \cap B=\emptyset$ and that $x \in\left[\mathbb{P}^{\star}(A \cup B)\right]_{\alpha}$.

Then there exists a function $f \in \mathcal{D}_{\alpha}$ such that $x=\int_{A \cup B} f(t) d \mu(t)$ and therefore

$$
\begin{aligned}
x & =\int_{A \cup B} f(t) d \mu(t)=\int_{A} f(t) d \mu(t)+\int_{B} f(t) d \mu(t) \epsilon \\
& \in\left[\mathbb{P}^{\star}(A)\right]_{\alpha} \oplus\left[\mathbb{P}^{\star}(B)\right]_{\alpha} .
\end{aligned}
$$

Der fifth point is a direct consequence of the fourth point.

In order to prove the last assertion of the theorem suppose that $A \cap B \neq \emptyset$ and that $x \in\left[\mathbb{P}^{\star}(A \cup B)\right]_{\alpha}$.

Then there exists a function $f \in \mathcal{D}_{\alpha}$ such that

$$
\begin{aligned}
x & =\int_{A \cup B} f(t) d \mu(t)=\int_{A \cap B^{c}} f(t) d \mu(t)+\int_{B \cap A^{c}} f(t) d \mu(t)+\int_{A \cap B} f(t) d \mu(t) \\
& =\int_{A} f(t) d \mu(t)+\int_{B} f(t) d \mu(t)-\int_{A \cap B} f(t) d \mu(t) \in \\
& \in\left[\mathbb{P}^{\star}(A)\right]_{\alpha} \oplus\left[\mathbb{P}^{\star}(B)\right]_{\alpha} \ominus\left[\mathbb{P}^{\star}(A \cap B)\right]_{\alpha} .
\end{aligned}
$$

In addition it is easy to calculate $\mathbb{P}^{\star}\left(A^{c}\right)$ if $\mathbb{P}^{\star}(A)$ is known.

Theorem 2.16 Let $(\Omega, \mathcal{A}, \mu)$ be an arbitrary measure space, $f^{\star}: \Omega \rightarrow \mathcal{F}_{c}^{1}$ a fuzzy probability density with respect to the measure $\mu, f^{\star}$ integrably bounded, and $\mathbb{P}^{\star}$ defined according to (2.14).

Then for $A, B \in \mathcal{A}$ the following assertions hold:

$$
\begin{aligned}
& \text { 1. } A \subseteq B \Longrightarrow\left[\mathbb{P}^{\star}(B \backslash A)\right]_{\alpha} \subseteq\left[\mathbb{P}^{\star}(B)\right]_{\alpha} \ominus\left[\mathbb{P}^{\star}(A)\right]_{\alpha} \quad \forall \alpha \in(0,1] \\
& \text { 2. } \mathbb{P}^{\star}\left(A^{c}\right)=\mathbf{1}_{\{1\}} \ominus \mathbb{P}^{\star}(A)
\end{aligned}
$$


Proof: Let $A \subseteq B$ and $x \in\left[\mathbb{P}^{\star}(B \backslash A)\right]_{\alpha}$, then by definition there exists $f \in \mathcal{D}_{\alpha}$ such that

$$
\begin{aligned}
x & =\int_{B \backslash A} f(t) d \mu(t)=\int_{B} f(t) d \mu(t)-\int_{A} f(t) d \mu(t) \epsilon \\
& \in\left[\mathbb{P}^{\star}(B)\right]_{\alpha} \ominus\left[\mathbb{P}^{\star}(A)\right]_{\alpha},
\end{aligned}
$$

which proves the first part of the theorem.

Suppose now that $x \in\left[\mathbb{P}^{\star}\left(A^{c}\right)\right]_{\alpha}$. Then there exists a function $f \in \mathcal{D}_{\alpha}$ with

$$
\begin{aligned}
x & =\int_{A^{c}} f(t) d \mu(t)=\int_{\Omega} f(t) d \mu(t)-\int_{A} f(t) d \mu(t) \\
& =1-\int_{A} f(t) d \mu(t) \in \\
& \in 1 \ominus\left[\mathbb{P}^{\star}(A)\right]_{\alpha} .
\end{aligned}
$$

On the other hand if $x \in 1 \ominus\left[\mathbb{P}^{\star}(A)\right]_{\alpha}$, then there exist $y \in\left[\mathbb{P}^{\star}(A)\right]_{\alpha}$ and $f \in \mathcal{D}_{\alpha}$ such that

$$
\begin{aligned}
x & =1-y=1-\int_{A} f(t) d \mu(t)=\int_{\Omega} f(t) d \mu(t)-\int_{A} f(t) d \mu(t) \\
& =\int_{\Omega \backslash A} f(t) d \mu(t) \in\left[\mathbb{P}^{\star}\left(A^{c}\right)\right]_{\alpha} .
\end{aligned}
$$

Remark: Note that according to Theorem 2.15 and Theorem $2.16 \mathbb{P}^{\star}(\cdot)$ especially fulfills all the properties of fuzzy relative frequencies $h_{n}^{\star}(\cdot)$ stated in Theorem 2.3.

In order to analyze properties of $\mathbb{P}^{\star}$ regarding countable unions of sets, the following common definition for a sequence $\left(I_{n}\right)_{n \in \mathbb{N}}$ of real intervals will be used:

$$
\sum_{n=1}^{\infty} I_{n}:=\left\{\sum_{n=1}^{\infty} y_{n}: \sum_{n=1}^{\infty}\left|y_{n}\right|<\infty \text { and } y_{n} \in I_{n} \forall n \in \mathbb{N}\right\}
$$

\section{Theorem 2.17 ( $\sigma$-Additivity)}

Let $(\Omega, \mathcal{A}, \mu)$ be an arbitrary measure space, $f^{\star}: \Omega \longrightarrow \mathcal{F}_{c}^{1}$ a fuzzy probability density with respect to the measure $\mu, f^{\star}$ integrably bounded, and the fuzzy probability distribution $\mathbb{P}^{\star}$ defined according to (2.14).

Furthermore suppose $\left(A_{n}\right)_{n \in \mathbb{N}}$ is a pairwise disjoint sequence of measurable sets, then for every $\alpha \in(0,1]$ it follows that

1. $[0,1] \cap \sum_{n=1}^{\infty}\left[\mathbb{P}^{\star}\left(A_{n}\right)\right]_{\alpha}$ is a compact interval and 
2. $\left[\mathbb{P}^{\star}\left(\bigcup_{n=1}^{\infty} A_{n}\right)\right]_{\alpha} \subseteq[0,1] \cap \sum_{n=1}^{\infty}\left[\mathbb{P}^{\star}\left(A_{n}\right)\right]_{\alpha}$.

Proof: Let $\left(A_{n}\right)_{n \in \mathbb{N}}$ be as in the theorem.

If $x \in\left[\mathbb{P}^{\star}\left(\bigcup_{n=1}^{\infty} A_{n}\right)\right]_{\alpha}$, then $x \in[0,1]$ and there exists a function $f \in \mathcal{D}_{\alpha}$ such that

$$
x=\int_{\bigcup_{n=1}^{\infty} A_{n}} f(t) d \mu(t)=\sum_{n=1}^{\infty} \int_{A_{n}} f(t) d \mu(t) \in \sum_{n=1}^{\infty}\left[\mathbb{P}^{\star}\left(A_{n}\right)\right]_{\alpha},
$$

whereby the second part of the theorem is proved.

To prove the fist part, write $\left[\mathbb{P}^{\star}\left(A_{n}\right)\right]_{\alpha}=:\left[a_{n}, b_{n}\right]$ and define

$$
[0,1] \cap \sum_{n=1}^{\infty}\left[\mathbb{P}^{\star}\left(A_{n}\right)\right]_{\alpha}=: I \text {. }
$$

Due to point two of the theorem it is clear that $\sum_{n=1}^{\infty} a_{n} \leq 1, I \neq \emptyset$. Using

$$
I=\left\{\sum_{n=1}^{\infty} x_{n} \leq 1: x_{n} \in\left[a_{n}, b_{n}\right] \forall n \in \mathbb{N}\right\}
$$

the convexity of $I$ is easy to see:

- If $\sum_{n=1}^{\infty} b_{n} \leq 1$ obviously $I=\left[\sum_{n=1}^{\infty} a_{n}, \sum_{n=1}^{\infty} b_{n}\right]$.

- If $\sum_{n=1}^{\infty} b_{n}>1$, then there exists a minimal $n_{0}$ such that $\sum_{k=1}^{n_{0}} b_{k}>1$. Define a new sequence $\left(c_{n}\right)_{n \in \mathbb{N}}$ by $c_{1}=b_{1}, c_{2}=b_{2}, \cdots, c_{n_{0}}=b_{n_{0}}, c_{n_{0}+1}=$ $a_{n_{0}+1}, c_{n_{0}+2}=a_{n_{0}+2}, \cdots$.

Because of $1<\sum_{n=1}^{\infty} c_{n}<\infty$ and $\sum_{n=1}^{\infty} a_{n} \leq 1$ there exists a real number $\lambda \in[0,1]$ such that

$$
\lambda \sum_{n=1}^{\infty} a_{n}+(1-\lambda) \sum_{n=1}^{\infty} c_{n}=1
$$

Therefore it follows that

$$
\sum_{n=1}^{n_{0}} \lambda a_{k}+(1-\lambda) b_{k}+\sum_{k=n_{0}}^{\infty} a_{k}=1
$$

and consequently $1 \in I$, which means that

$$
I=\left[\sum_{n=1}^{\infty} a_{n}, 1\right]
$$


Before continuing and proposing a general notion of a fuzzy probability distribution the $\alpha$-cut representation of $\mathbb{P}^{\star}(A)$, shortly mentioned before Theorem 2.12 , will be picked up again. It will be especially helpful in order to see the similarity of fuzzy probability distributions induced by fuzzy probability densities as presented before and that of fuzzy probability distributions induced by fuzzy random variables (compare Section 4.3).

As hitherto let $(\Omega, \mathcal{A}, \mu)$ be an arbitrary measure space, $f^{\star}: \Omega \longrightarrow \mathcal{F}_{c}^{1}$ a fuzzy probability density with respect to the measure $\mu, f^{\star}$ integrably bounded, and the fuzzy probability distribution $\mathbb{P}^{\star}$ defined according to (2.14).

Remember that the boundaries $\underline{p}_{-\alpha}, \bar{p}_{\alpha}$ of the $\alpha$-cuts $\left[\mathbb{P}^{*}(A)\right]_{\alpha}$ are real functions $\underline{p}_{\alpha}, \bar{p}_{\alpha}: \mathcal{A} \rightarrow \mathbb{R}$, defined by

$$
\left[\underline{p}_{\alpha}(A), \bar{p}_{\alpha}(A)\right]:=\left[\mathbb{P}^{\star}(A)\right]_{\alpha}, \quad \text { where } \alpha \in(0,1] \text { and } A \in \mathcal{A},
$$

or formulated explicitly

$\underline{p}_{\alpha}(A)=\min \left[\mathbb{P}^{\star}(A)\right]_{\alpha}, \bar{p}_{\alpha}(A)=\max \left[\mathbb{P}^{\star}(A)\right]_{\alpha}, \quad$ where $\alpha \in(0,1]$ and $A \in \mathcal{A}$.

Having this it follows immediately from the last three theorems, that the setfunctions $\underline{p}_{\alpha}, \bar{p}_{\alpha}$ have (amongst others) the following properties summarized in the next theorem.

Theorem 2.18 Let $(\Omega, \mathcal{A}, \mu)$ be an arbitrary measure space, $f^{\star}: \Omega \rightarrow \mathcal{F}_{c}^{1}$ a fuzzy probability density with respect to the measure $\mu, f^{\star}$ integrably bounded, $\mathbb{P}^{\star}$ defined according to (2.14) and $\underline{p}_{\alpha}, \bar{p}_{\alpha}$ defined according to (2.22).

Then the following assertions hold:

1. $\underline{p}_{\alpha}(\Omega)=\bar{p}_{\alpha}(\Omega)=1, \quad \underline{p}_{\alpha}(\emptyset)=\bar{p}_{\alpha}(\emptyset)=0 \quad \forall \alpha \in(0,1]$.

2. If $A, B \in \mathcal{A}, A \subseteq B$, then $\underline{p}_{\alpha}(A) \leq \underline{p}_{\alpha}(B)$ and $\bar{p}_{\alpha}(A) \leq \bar{p}_{\alpha}(B)$ holds for all $\alpha \in(0,1]$.

3. For every $\alpha \in(0,1] p_{\alpha}$ is superadditive and $\bar{p}_{\alpha}$ is subadditive, i.e. if $A, B \in \mathcal{A}$ and $A \cap \bar{B}^{\alpha}=\emptyset$, then $\underline{p}_{\alpha}(A \cup B) \geq \underline{p}_{\alpha}(A)+\underline{p}_{\alpha}(B)$ and $\bar{p}_{\alpha}(A \cup B) \leq \bar{p}_{\alpha}(A)+\bar{p}_{\alpha}(B)$ holds.

4. For every $\alpha \in(0,1] \underline{p}_{\alpha}$ is even super- $\sigma$-additive and $\bar{p}_{\alpha}$ is sub- $\sigma$ additive, i.e. if $\left(A_{n}\right)_{n \in \mathbb{N}}$ is a sequence of pairwise disjoint, measurable sets then the following inequalities hold for every $\alpha \in(0,1]$ :

$$
\begin{aligned}
& \bar{p}_{\alpha}\left(\bigcup_{n=1}^{\infty} A_{n}\right) \leq \sum_{n=1}^{\infty} \bar{p}_{\alpha}\left(A_{n}\right) \\
& \underline{p}_{\alpha}\left(\bigcup_{n=1}^{\infty} A_{n}\right) \geq \sum_{n=1}^{\infty} \underline{p}_{\alpha}\left(A_{n}\right)
\end{aligned}
$$


5. For $A \in \mathcal{A}$ the following equalities hold:

$$
\bar{p}_{\alpha}\left(A^{c}\right)=1-\underline{p}_{\alpha}(A) \quad \text { and } \quad \underline{p}_{\alpha}\left(A^{c}\right)=1-\bar{p}_{\alpha}(A)
$$

\subsection{General fuzzy probability distributions}

Looking back at the properties of fuzzy relative frequencies stated in Theorem 2.3 and that of fuzzy probability distributions induced by integrably bounded fuzzy probability densities as formulated in Theorem 2.15 and Theorem 2.16, then the following definition of a general fuzzy probability distribution is suggestive:

Definition 2.19 Suppose that $\mathcal{A}$ is a $\sigma$-algebra in $\Omega$, then a fuzzy-valued function $\mathbb{P}^{\star}: \mathcal{A} \rightarrow \mathcal{F}_{c}^{1}$ is called (general) fuzzy probability distribution on $\Omega$, if the following four conditions are fulfilled:

1. $\mathbb{P}^{\star}(\Omega)=1_{\{1\}}, \quad \mathbb{P}^{\star}(\emptyset)=1_{\{0\}}$

2. If $A, B \in \mathcal{A}, A \subseteq B$, then $\mathbb{P}^{\star}(A) \preceq \mathbb{P}^{\star}(B)$ holds.

3. If $A, B \in \mathcal{A}, A \cap B=\emptyset$, then $\mathbb{P}^{\star}(A \cup B) \subseteq \mathbb{P}^{\star}(A) \oplus \mathbb{P}^{\star}(B)$ holds.

4. If $A \in \mathcal{A}$, then $\mathbb{P}^{\star}\left(A^{c}\right)=1_{\{1\}} \ominus \mathbb{P}^{\star}(A)$

Again considering the $\alpha$-cuts $\left[\underline{p}_{\alpha}, \bar{p}_{\alpha}\right]$ of $\mathbb{P}^{\star}: \mathcal{A} \rightarrow \mathcal{F}_{c}^{1}$, defined by

$$
\left[\underline{p}_{\alpha}(A), \bar{p}_{\alpha}(A)\right]:=\left[\mathbb{P}^{\star}(A)\right]_{\alpha}, \quad \text { where } \alpha \in(0,1] \text { and } A \in \mathcal{A},
$$

Definition 2.19 can be reformulated equivalently as follows:

Definition 2.20 Suppose that $\mathcal{A}$ is a $\sigma$-algebra in $\Omega$, then a fuzzy-valued function $\mathbb{P}^{\star}: \mathcal{A} \rightarrow \mathcal{F}_{c}^{1}$ is called (general) fuzzy probability distribution on $\Omega$, if the $\alpha$-cuts $\left[\underline{p}_{\alpha}(\cdot), \bar{p}_{\alpha}(\cdot)\right]$ defined according to (2.23) fulfill the following four conditions:

1. $\underline{p}_{\alpha}(\Omega)=\bar{p}_{\alpha}(\Omega)=1, \quad \underline{p}_{\alpha}(\emptyset)=\bar{p}_{\alpha}(\emptyset)=0 \quad \forall \alpha \in(0,1]$

2. If $A, B \in \mathcal{A}, A \subseteq B$, then $\underline{p}_{\alpha}(A) \leq \underline{p}_{\alpha}(B)$ and $\bar{p}_{\alpha}(A) \leq \bar{p}_{\alpha}(B)$ holds for all $\alpha \in(0,1]$.

3. For every $\alpha \in(0,1] p_{\alpha}$ is superadditive and $\vec{p}_{\alpha}$ is subadditive, i.e. if $A, B \in \mathcal{A}$ and $A \cap \bar{B}=\emptyset$, then $\underline{p}_{\alpha}(A \cup B) \geq \underline{p}_{\alpha}(A)+\underline{p}_{\alpha}(B)$ and $\bar{p}_{\alpha}(A \cup B) \leq \bar{p}_{\alpha}(A)+\bar{p}_{\alpha}(B)$ holds. 
4. For every $A \in \mathcal{A}$ and every $\alpha \in(0,1]$ the identities $\underline{p}_{\alpha}\left(A^{c}\right)=1-\bar{p}_{\alpha}(A)$ and $\bar{p}_{\alpha}\left(A^{c}\right)=1-\underline{p}_{\alpha}(A)$ hold.

Remark: It is clear that every probability measure $\mathcal{P}$ on $(\Omega, \mathcal{A})$ can be seen as a fuzzy probability distribution by simply defining $\mathbb{P}^{\star}(A):=\mathbf{1}_{\{\mathcal{P}(A)\}} \in \mathcal{F}_{c}^{1}$. 


\section{Chapter 3}

\section{Expectation and other characteristics}

\subsection{Discrete case}

Suppose $\Omega=\left\{a_{1}, \cdots, a_{n}\right\} \subset \mathbb{R}$ is a finite set and $\mathbb{P}^{\star}: 2^{\Omega} \longrightarrow \mathcal{F}_{c}^{1}$ is a discrete fuzzy probability distribution on $\Omega$ induced by a discrete fuzzy probability density $p^{\star}$. It is natural to ask questions about if and how the concepts of characteristics like expectation or other moments can be applied to the concept of fuzzy probability distributions. Intuitively these characteristics should be fuzzy numbers as well.

Continuing the same ideas as before the following definitions for the expectation $m_{1}^{\star}$ and the $k$-th central moment $\tilde{m}_{k}^{\star}(k \geq 1)$ seem to be natural:

$$
\begin{aligned}
{\left[m_{1}^{\star}\right]_{\alpha}: } & =\left\{\sum_{i=1}^{n} a_{i} x_{i}: \quad x=\left(x_{1}, x_{2}, \cdots, x_{n}\right) \in \mathcal{D}_{\alpha}\right\} \\
{\left[\tilde{m}_{k}^{\star}\right]_{\alpha}: } & =\left\{\sum_{i=1}^{n}\left(a_{i}-\sum_{j=1}^{n} a_{j} x_{j}\right)^{k} x_{i}: \quad x=\left(x_{1}, x_{2}, \cdots, x_{n}\right) \in \mathcal{D}_{\alpha}\right\}
\end{aligned}
$$

Thereby $\alpha \in(0,1]$ and

$\mathcal{D}_{\alpha}=\left\{x=\left(x_{1}, \cdots, x_{n}\right) \in \mathbb{R}^{n}: x_{i} \in\left[p_{i}^{\star}\right]_{\alpha} \quad \forall i \in\{1, \cdots, n\}\right.$ and $\left.\sum_{i=1}^{n} x_{i}=1\right\}$.

Lemma 3.1 Let $\Omega=\left\{a_{1}, \cdots, a_{n}\right\} \subset \mathbb{R}$ be a finite set and $\mathbb{P}^{\star}: 2^{\Omega} \longrightarrow \mathcal{F}_{c}^{1}$ a discrete fuzzy probability distribution on $\Omega$ induced by a discrete fuzzy probability density $p^{\star}$. Furthermore let $\left[m_{1}^{\star}\right]_{\alpha}$ for $\alpha \in(0,1]$ be defined according to (3.1), then: 
1. $\left[m_{1}^{\star}\right]_{\alpha}$ is a compact interval for every $\alpha \in(0,1]$,

2. $\left[m_{1}^{\star}\right]_{\alpha}$ is a nested family monotonically decreasing in $\alpha$ with

$$
\bigcap_{\alpha<\beta}\left[m_{1}^{\star}\right]_{\alpha}=\left[m_{1}^{\star}\right]_{\beta}
$$

Proof: The mapping $f: \mathbb{R}^{n} \longrightarrow \mathbb{R}$, defined by

$$
f\left(\left(x_{1}, \cdots, x_{n}\right)\right):=\sum_{i=1}^{n} a_{i} x_{i},
$$

is linear, continuous and satisfies

$$
f\left(\mathcal{D}_{\alpha}\right)=\left[m_{1}^{\star}\right]_{\alpha}
$$

Therefore it immediately follows that $\left[m_{1}^{\star}\right]_{\alpha}$ is a compact interval.

Since $\mathcal{D}_{\alpha}$ monotonically decreases as $\alpha$ increases it is clear that $\left[m_{1}^{\star}\right]_{\alpha}$ is monotonically decreasing in $\alpha$ too.

Suppose now that

$$
z \in \bigcap_{\alpha<\beta}\left[m_{1}^{\star}\right]_{\alpha}
$$

and denote by $\left(\alpha_{k}\right)_{k \in \mathbb{N}}$ a strictly increasing sequence of positive real numbers converging to $\beta$.

Then $z \in\left[m_{1}^{\star}\right]_{\alpha_{k}} \forall k \in \mathbb{N}$ and for every $k$ there exists a $x_{k} \in \mathcal{D}_{\alpha_{k}}$ such that

$$
f\left(x_{k}\right)=z .
$$

Since $\mathcal{D}_{\alpha}$ is compact for every $\alpha \in(0,1]$ and since $x_{k} \in \mathcal{D}_{\alpha_{1}}$ for all $k \in \mathbb{N}$ there exists a convergent subsequence $\left(x_{k_{l}}\right)_{l \in \mathbb{N}}$ converging to a point $x$. It is easy to see that $x \in \mathcal{D}_{\alpha_{k}}$ for every $k$ and therefore

$$
x \in \bigcap_{k=1}^{\infty} \mathcal{D}_{\alpha_{k}}=\mathcal{D}_{\beta} .
$$

Due to the continuity of $f$ it follows that $f(x)=z$, which implies that

$$
z \in\left[m_{1}^{\star}\right]_{\beta} .
$$

The other inclusion is obvious.

Lemma 3.1 shows that the family $\left(\left[m_{1}^{\star}\right]_{\alpha}\right)_{\alpha \in(0,1]}$ is a family of $\alpha$-cuts of a fuzzy number. Consequently the following definition makes sense: 
Definition 3.2 Suppose $\Omega=\left\{a_{1}, \cdots, a_{n}\right\} \subset \mathbb{R}$ is a finite set and that $\mathbb{P}^{\star}: 2^{\Omega} \longrightarrow \mathcal{F}_{c}^{1}$ is a discrete fuzzy probability distribution on $\Omega$ induced by a discrete fuzzy probability density $p^{\star}$. Then the fuzzy number $m_{1}^{\star}$, defined via its $\alpha$-cuts

$$
\left[m_{1}^{\star}\right]_{\alpha}:=\left\{\sum_{i=1}^{n} a_{i} x_{i}: \quad x=\left(x_{1}, x_{2}, \cdots, x_{n}\right) \in \mathcal{D}_{\alpha}\right\},
$$

is called the expectation of the discrete fuzzy probability distribution $\mathbb{P}^{\star}$.

Lemma 3.3 Let $\Omega=\left\{a_{1}, \cdots, a_{n}\right\} \subset \mathbb{R}$ be a finite set and $\mathbb{P}^{\star}: 2^{\Omega} \longrightarrow \mathcal{F}_{c}^{1} a$ discrete fuzzy probability distribution on $\Omega$ induced by a discrete fuzzy probability density $p^{\star}$. Furthermore let $\left[\tilde{m}_{k}^{\star}\right]_{\alpha}$ for $k \geq 1$ be defined according to (3.2), then:

1. $\left[\tilde{m}_{k}^{\star}\right]_{\alpha}$ is a compact interval for every $\alpha \in(0,1]$,

2. $\left[\tilde{m}_{k}^{\star}\right]_{\alpha}$ is a nested family monotonically decreasing in $\alpha$ with

$$
\bigcap_{\alpha<\beta}\left[\tilde{m}_{k}^{\star}\right]_{\alpha}=\left[\tilde{m}_{k}^{\star}\right]_{\beta}
$$

Proof: The mapping $f_{k}: \mathbb{R}^{n} \longrightarrow \mathbb{R}$, defined by

$$
f_{k}\left(\left(x_{1}, \cdots, x_{n}\right)\right)=\sum_{i=1}^{n}\left(a_{i}-\sum_{j=1}^{n} a_{j} x_{j}\right)^{k} x_{i},
$$

is continuous and satisfies

$$
f_{k}\left(\mathcal{D}_{\alpha}\right)=\left[\tilde{m}_{k}^{\star}\right]_{\alpha}
$$

Since $\mathcal{D}_{\alpha} \subseteq \mathbb{R}^{n}$ is compact for each $\alpha \in(0,1]$ it follows immediately that $\left[\tilde{m}_{k}^{\star}\right]_{\alpha}$ is a compact interval.

As $\mathcal{D}_{\alpha}$ is monotonically decreasing as $\alpha$ increases it is clear that $\left[\tilde{m}_{k}^{\star}\right]_{\alpha}$ is monotonically decreasing in $\alpha$ too.

Suppose now that

$$
z \in \bigcap_{\alpha<\beta}\left[\tilde{m}_{k}^{\star}\right]_{\alpha}
$$

and denote by $\left(\alpha_{j}\right)_{j \in \mathbb{N}}$ a strictly increasing sequence of positive real numbers converging to $\beta$.

Then $z \in\left[\tilde{m}_{k}^{\star}\right]_{\alpha_{j}} \forall j \in \mathbb{N}$ and for every $j$ there exists by definition a point $x_{j} \in \mathcal{D}_{\alpha_{j}}$ such that

$$
f_{k}\left(x_{j}\right)=z
$$


Since $\mathcal{D}_{\alpha}$ is compact for every $\alpha \in(0,1]$ and $x_{j} \in \mathcal{D}_{\alpha_{1}}$ for all $j \in \mathbb{N}$ there exists a convergent subsequence $\left(x_{j_{l}}\right)_{l \in \mathbb{N}}$ converging to a point $x$. It is easy to see that $x \in \mathcal{D}_{\alpha_{j}}$ for every $j$ and therefore

$$
x \in \bigcap_{j=1}^{\infty} \mathcal{D}_{\alpha_{j}}=\mathcal{D}_{\beta}
$$

Due to the continuity of $f_{k}$ it follows that $f_{k}(x)=z$, which proves that

$$
x \in\left[\tilde{m}_{k}^{\star}\right]_{\beta}
$$

The other inclusion is obvious.

As before the above Lemma 3.3 justifies the following definition:

Definition 3.4 Suppose $\Omega=\left\{a_{1}, \cdots, a_{n}\right\} \subset \mathbb{R}$ is a finite set and that $\mathbb{P}^{\star}: 2^{\Omega} \longrightarrow \mathcal{F}_{c}^{1}$ is a discrete fuzzy probability distribution on $\Omega$ induced by a discrete fuzzy probability density $p^{\star}$. Then the fuzzy number $\tilde{m}_{k}^{\star}$, defined via its $\alpha$-cuts

$$
\left[\tilde{m}_{k}^{\star}\right]_{\alpha}:=\left\{\sum_{i=1}^{n}\left(a_{i}-\sum_{j=1}^{n} a_{j} x_{j}\right)^{k} x_{i}: \quad x=\left(x_{1}, x_{2}, \cdots, x_{n}\right), \in \mathcal{D}_{\alpha}\right\}
$$

is called the $k$-th central moment of the discrete fuzzy probability distribution $\mathbb{P}^{\star}$ on $\Omega$.

\subsection{Case of fuzzy probability distributions in- duced by fuzzy probability densities}

Let $(\Omega, \mathcal{A}, \mu)=(\mathbb{R}, \mathcal{B}(\mathbb{R}), \mu)$, where $\mathcal{B}(\mathbb{R})$ denotes the Borel sets on $\mathbb{R}$ and $\mu$ denotes an arbitrary measure on $\mathcal{B}(\mathbb{R})$.

Furthermore suppose that $f^{\star}: \mathbb{R} \longrightarrow \mathcal{F}_{c}^{1}$ is a fuzzy probability density with respect to the measure $\mu$ and integrably bounded by a function $h \in$ $L^{1}(\mathbb{R}, \mathcal{B}(\mathbb{R}), \mu)$.

As in the previous section $\mathbb{P}^{\star}$ is defined according to (2.14).

Following the ideas used before, a natural extension $m_{1}^{\star}$ of the concept of expectation is defined via its $\alpha$-cuts by

$$
\left[m_{1}^{\star}\right]_{\alpha}:=\left\{\int_{\mathbb{R}} t f(t) d \mu(t): f \in \mathcal{D}_{\alpha}\right\}, \quad \alpha \in(0,1] .
$$


In order to ensure the existence of the above integrals, suppose that

$$
\int_{\mathbb{R}}|t h(t)| d \mu(t)<\infty .
$$

Using the abbreviation

$$
\mathcal{E}_{1}^{\alpha}:=\left\{g(t)=t f(t): f \in \mathcal{D}_{\alpha}\right\}
$$

the above definition reduces to

$$
\left[m_{1}^{\star}\right]_{\alpha}=\left\{\int_{\mathbb{R}} g(t) d \mu(t): g \in \mathcal{E}_{1}^{\alpha}\right\}, \quad \alpha \in(0,1] .
$$

The procedure for showing that (3.3) under suitable assumptions determines a family of $\alpha$-cuts of a fuzzy number is similar to the procedure in the foregoing chapter, in which the first step was to prove that $\mathcal{D}_{\alpha}$ is a weakly compact subset of $L^{1}(\Omega, \mathcal{A}, \mu)$.

Now the first step is to show that $\mathcal{E}_{1}^{\alpha}$ has the same property.

Theorem 3.5 Suppose $\mu$ is a measure on the Borel subsets $\mathcal{B}(\mathbb{R})$ of $\mathbb{R}$, $f^{\star}: \Omega \longrightarrow \mathcal{F}_{c}^{1}$ is a fuzzy probability density with respect to the measure $\mu$, and $f^{\star}$ is integrably bounded by a function $h \in L^{1}(\mathbb{R}, \mathcal{B}(\mathbb{R}), \mu)$. Furthermore suppose that $\int_{\mathbb{R}}|t h(t)| d \mu(t)<\infty$.

Then $\mathcal{E}_{1}^{\alpha}$ is a weakly compact subset of $L^{1}(\mathbb{R}, \mathcal{B}(\mathbb{R}), \mu)$ for every $\alpha \in(0,1]$.

Proof: Because of $\int_{\mathbb{R}}|t h(t)| d \mu(t)<\infty$ it follows that

$$
\int_{\mathbb{R}}|t f(t)| d \mu(t)<\infty \quad \forall f \in \mathcal{D}_{\alpha}
$$

holds and therefore that $\mathcal{E}_{1}^{\alpha} \subseteq L^{1}(\mathbb{R}, \mathcal{B}(\mathbb{R}), \mu)$ for every $\alpha \in(0,1]$.

Defining $\tilde{h}(t):=t h(t)$ for all $t \in \mathbb{R}$ it follows that for arbitrary $g \in \mathcal{E}_{1}^{\alpha}$

$$
|g(t)| \leq|\tilde{h}(t)| \text { for } \mu \text {-almost every } t \in \mathbb{R} \text {. }
$$

Using this and following the idea of the proof of Theorem 2.9 it is easy to prove that the family $\mathcal{E}_{1}^{\alpha}$ fulfills the points of Theorem 6.11 in the Appendix and is therefore weakly precompact for every $\alpha \in(0,1]$.

Because of the fact, that $\mathcal{E}_{1}^{\alpha}$ inherits the convexity from $\mathcal{D}_{\alpha}$ the Theorem of Mazur (Theorem 6.12) shows that the weak closure of $\mathcal{E}_{1}^{\alpha}$ is equal to its strong closure. If it can be proved that $\mathcal{E}_{1}^{\alpha}$ is closed in the strong topology it will therefore follow that it is weakly closed, which together with the already proved weak precompactness proves the compactness of $\mathcal{E}_{1}^{\alpha}$ in the weak topology. 
In this regard suppose $\left(g_{n}\right)_{n \in \mathbb{N}}$ is a strongly convergent sequence in $\mathcal{E}_{1}^{\alpha}$, with limit function $g \in L^{1}(\mathbb{R}, \mathcal{B}(\mathbb{R}), \mu)$. Then there exists a $\mu$-almost everywhere to $g$ convergent subsequence $\left(g_{n_{k}}\right)_{k \in \mathbb{N}}$.

Therefore

$$
\frac{g_{n_{k}}(t)}{t} \longrightarrow \frac{g(t)}{t} \text { as } k \rightarrow \infty \text { for } \mu \text {-almost every } t \in \mathbb{R}
$$

Because of

$$
\frac{g_{n_{k}}(t)}{t} \in F_{\alpha}(t) \text { for } \mu \text {-almost every } t \in \mathbb{R}
$$

and the compactness of $F_{\alpha}(t)$ it follows that

$$
\frac{g(t)}{t} \in F_{\alpha}(t) \text { for } \mu \text {-almost every } t \in \mathbb{R} \text {. }
$$

Using the fact that

$$
\left|\frac{g_{n_{k}}(t)}{t}\right| \leq|\tilde{h}(t)| \text { for all } k \in \mathbb{N},
$$

together with Lebesgue's Dominated Convergence Theorem yields

$$
1=\int_{\mathbb{R}} \frac{g_{n_{k}}(t)}{t} d \mu(t) \longrightarrow \int_{\mathbb{R}} \frac{g(t)}{t} d \mu(t) \quad \text { for } k \rightarrow \infty,
$$

which shows that $\frac{g(t)}{t} \in \mathcal{D}_{\alpha}$, and therefore $g(t) \in \mathcal{E}_{1}^{\alpha}$.

Theorem 3.6 Suppose $\mu$ is a measure on the Borel subsets $\mathcal{B}(\mathbb{R})$ of $\mathbb{R}$, $f^{\star}: \Omega \longrightarrow \mathcal{F}_{c}^{1}$ is a fuzzy probability density with respect to the measure $\mu$, and $f^{\star}$ is integrably bounded by a function $h \in L^{1}(\mathbb{R}, \mathcal{B}(\mathbb{R}), \mu)$.

Furthermore suppose that $\int_{\mathbb{R}}|t h(t)| d \mu(t)<\infty$ and let $\left[m_{1}^{\star}\right]_{\alpha}$ for $\alpha \in(0,1]$ be defined according to (3.3), then

1. $\left[m_{1}^{\star}\right]_{\alpha}$ is a compact interval for every $\alpha \in(0,1]$

2. $\left[m_{1}^{\star}\right]_{\alpha}$ is a nested monotonically decreasing family in $\alpha$ with

$$
\bigcap_{\alpha<\beta}\left[m_{1}^{\star}\right]_{\alpha}=\left[m_{1}^{\star}\right]_{\beta}
$$

Proof: The mapping $\Psi$, defined by

$$
\begin{aligned}
\Psi: L^{1}(\mathbb{R}, \mathcal{B}(\mathbb{R}, \mu) & \longrightarrow \mathbb{R} \\
g & \longmapsto \int_{\mathbb{R}} g(t) d \mu(t),
\end{aligned}
$$


is a continuous linear functional on $L^{1}(\Omega, \mathcal{A}, \mu)$ and therefore also continuous in the weak topology. Moreover it satisfies

$$
\Psi\left(\mathcal{E}_{1}^{\alpha}\right)=\left[m_{1}^{\star}\right]_{\alpha} \quad \forall \alpha \in(0,1] .
$$

$\Psi$ perserves compactness and convexity, which proves the compactness and convexity of $\left[m_{1}^{\star}\right]_{\alpha}$ and completes the proof of the first part of the theorem. Since $\mathcal{E}_{1}^{\alpha}$ decreases as $\alpha$ increases, $\left[m_{1}^{\star}\right]_{\alpha}$ is decreasing in $\alpha$ too.

It remains to show that

$$
\bigcap_{\alpha<\beta}\left[m_{1}^{\star}\right]_{\alpha}=\left[m_{1}^{\star}\right]_{\beta} .
$$

Suppose $x \in\left[m_{1}^{\star}\right]_{\alpha}$ for all $\alpha<\beta$ and $\left(\alpha_{n}\right)_{n \in \mathbb{N}}$ is a strictly increasing sequence of positive real numbers converging to $\beta$.

Then for every $n \in \mathbb{N}$ there exists a function $g_{n} \in \mathcal{E}_{1}^{\alpha_{n}}$ such that

$$
\int_{\mathbb{R}} g_{n}(t) d \mu(t)=x
$$

Because $g_{n} \in \mathcal{E}^{\alpha_{1}}$ holds for all $n \in \mathbb{N}$ and the because of the fact that $\mathcal{E}_{1}^{\alpha}$ is weakly compact for every $\alpha \in(0,1]$, it follows that there exists a function $g \in \mathcal{E}_{1}^{\alpha_{1}}$ and a subsequence $\left(g_{n_{k}}\right)_{k \in \mathbb{N}}$ such that

$$
g_{n_{k}} \longrightarrow g \quad \text { weakly for } k \rightarrow \infty \text {. }
$$

Since for each $j$ almost all $g_{n_{k}}$ lie in $\mathcal{E}_{1}^{\alpha_{j}}$ it follows using the same argument that $g \in \mathcal{E}_{1}^{\alpha_{j}}$.

Using

$$
\bigcap_{n=1}^{\infty} F_{\alpha_{n}}(t)=F_{\beta}(t)
$$

one sees that $f(t):=\frac{g(t)}{t} \in F_{\beta}(t) \mu$-almost everywhere and therefore $\frac{g(t)}{t} \in$ $\mathcal{D}_{\beta}$. This shows that $x \in\left[m_{1}^{\star}\right]_{\beta}$ and

$$
\bigcap_{\alpha<\beta}\left[m_{1}^{\star}\right]_{\alpha} \subseteq\left[m_{1}^{\star}\right]_{\beta}
$$

The other inclusion is obvious.

The above theorem justifies the following definition:

Definition 3.7 Suppose $\mu$ is a measure on the Borel subsets $\mathcal{B}(\mathbb{R})$ of $\mathbb{R}$, $f^{\star}: \Omega \longrightarrow \mathcal{F}_{c}^{1}$ is a fuzzy probability density with respect to the measure $\mu$, and $f^{\star}$ is integrably bounded by a function $h \in L^{1}(\mathbb{R}, \mathcal{B}(\mathbb{R}), \mu)$.

Furthermore suppose that $\int_{\mathbb{R}}|t h(t)| d \mu(t)<\infty$ and define the fuzzy probability 
distribution $\mathbb{P}^{\star}$ according to (2.14).

Then the fuzzy number $m_{1}^{\star}$, defined via its $\alpha$-cuts

$$
\left[m_{1}^{\star}\right]_{\alpha}:=\left\{\int_{\mathbb{R}} t f(t) d \mu(t): g \in \mathcal{D}_{\alpha}\right\}, \quad \alpha \in(0,1]
$$

is called expectation of the fuzzy probability distribution $\mathbb{P}^{\star}$ on $\mathbb{R}$.

Following the ideas used before, a natural extension $m_{k}^{\star}$ of the concept of the $k$-th moment is

$$
\left[m_{k}^{\star}\right]_{\alpha}:=\left\{\int_{\mathbb{R}} t^{k} f(t) d \mu(t): f \in \mathcal{D}_{\alpha}\right\}, \quad \alpha \in(0,1] .
$$

In order to ensure the existence of the above integrals, suppose now that

$$
\int_{\mathbb{R}}\left|t^{k} h(t)\right| d \mu(t)<\infty
$$

Using the abbreviation

$$
\mathcal{E}_{k}^{\alpha}:=\left\{g(t)=t^{k} f(t): f \in \mathcal{D}_{\alpha}\right\}
$$

the above definition reduces to

$$
\left[m_{k}^{\star}\right]_{\alpha}=\left\{\int_{\mathbb{R}} g(t) d \mu(t): g \in \mathcal{E}_{k}^{\alpha}\right\}, \quad \alpha \in(0,1] .
$$

Note that in the general case in contrast to the discrete case the $k$-th moment and not the $k$-th central moment is defined.

Theorem 3.8 Suppose $\mu$ is a measure on the Borel subsets $\mathcal{B}(\mathbb{R})$ of $\mathbb{R}$, $f^{\star}: \Omega \longrightarrow \mathcal{F}_{c}^{1}$ is a fuzzy probability density with respect to the measure $\mu$, and $f^{\star}$ is integrably bounded by a function $h \in L^{1}(\mathbb{R}, \mathcal{B}(\mathbb{R}), \mu)$. Furthermore suppose that $\int_{\mathbb{R}}\left|t^{k} h(t)\right| d \mu(t)<\infty$.

Then $\mathcal{E}_{k}^{\alpha}$ is a weakly compact subset of $L^{1}(\mathbb{R}, \mathcal{B}(\mathbb{R}), \mu)$ for every $\alpha \in(0,1]$.

Proof: The theorem is easily proved by following the proof of Theorem 3.5 and replacing $\mathcal{E}_{1}^{\alpha}$ by $\mathcal{E}_{k}^{\alpha}$.

Theorem 3.9 Suppose $\mu$ is a measure on the Borel subsets $\mathcal{B}(\mathbb{R})$ of $\mathbb{R}$, $f^{\star}: \Omega \longrightarrow \mathcal{F}_{c}^{1}$ is a fuzzy probability density with respect to the measure $\mu$, and $f^{\star}$ is integrably bounded by a function $h \in L^{1}(\mathbb{R}, \mathcal{B}(\mathbb{R}), \mu)$.

Furthermore suppose that $\int_{\mathbb{R}}\left|t^{k} h(t)\right| d \mu(t)<\infty$ and let $\left[m_{k}^{\star}\right]_{\alpha}$ for $\alpha \in(0,1]$ be defined according to (3.4), then 
1. $\left[m_{k}^{\star}\right]_{\alpha}$ is a compact interval for every $\alpha \in(0,1]$

2. $\left[m_{k}^{\star}\right]_{\alpha}$ is a nested family monotonically decreasing in $\alpha$ with

$$
\bigcap_{\alpha<\beta}\left[m_{k}^{\star}\right]_{\alpha}=\left[m_{k}^{\star}\right]_{\beta}
$$

Proof: It is obvious how to prove the theorem by following the proof of Theorem 3.6 and replacing $\mathcal{E}_{1}^{\alpha}$ by $\mathcal{E}_{k}^{\alpha}$ and $\left[m_{1}^{\star}\right]_{\alpha}$ by $\left[m_{k}^{\star}\right]_{\alpha}$.

The above theorem justifies the following definition:

Definition 3.10 Suppose $\mu$ is a measure on the Borel subsets $\mathcal{B}(\mathbb{R})$ of $\mathbb{R}$, $f^{\star}: \Omega \longrightarrow \mathcal{F}_{c}^{1}$ is a fuzzy probability density with respect to the measure $\mu$, and $f^{\star}$ is integrably bounded by a function $h \in L^{1}(\mathbb{R}, \mathcal{B}(\mathbb{R}), \mu)$.

Furthermore suppose that $\int_{\mathbb{R}}\left|t^{k} h(t)\right| d \mu(t)<\infty$ and define the fuzzy probability distribution $\mathbb{P}^{\star}$ according to (2.14).

Then the fuzzy number $m_{k}^{\star}$, defined via its $\alpha$-cuts

$$
\left[m_{k}^{\star}\right]_{\alpha}:=\left\{\int_{\mathbb{R}} t^{k} f(t) d \mu(t): g \in \mathcal{D}_{\alpha}\right\}, \quad \alpha \in(0,1],
$$

is called $k$-th moment of the fuzzy probability distribution $\mathbb{P}^{\star}$ on $\mathbb{R}$. 


\section{Chapter 4}

\section{Fuzzy random variables and vectors}

In the last decades of the 20th century fuzzy random variables (and fuzzy random vectors) were a particular subject of research since they combine both randomness (stochastic uncertainty) and fuzziness (imprecision), where imprecision means non-statistical uncertainty due to the inaccuracy of human knowlegde or the inexactness of measurements.

In short a fuzzy random variable is a fuzzy-valued mapping defined on a probability space, fulfilling a certain measurability condition similar to the classical case of (real-valued) random variables. Concerning the precise measurability condition there is no generally accepted concept but mainly three different approaches have been developed:

Kwakernaak [21] focused on the corresponding $\alpha$-cut mappings (see below) assuming that the lower and upper boundary functions are Borel measurable. Puri and Ralescu [33] also considered the induced $\alpha$-cut mappings and postulated that these mappings are random compact sets (compare [29]).

Klement et al. [18] called a fuzzy-valued mapping on a probability space a fuzzy random variable if it is measurable with respect to the Borel $\sigma$-algebra induced by certain metrics defined on (subsets of) $\mathcal{F}_{c}^{1}$.

In the following at first different measurability conditions for fuzzy random variables and their interrelation will be analyzed in detail. After that fuzzy random vectors, which are the multi-dimensional analogy of fuzzy random variables, and their corresponding measurability conditions will be discussed briefly (since the results and proofs are very similar).

Finally it will be shown how fuzzy random variables and fuzzy random vectors naturally induce a fuzzy probability distribution according to Defintion 2.19 on the Borel $\sigma$-algebra $\mathcal{B}(\mathbb{R})$ in $\mathbb{R}$ and the Borel $\sigma$-algebra $\mathcal{B}\left(\mathbb{R}^{d}\right)$ in $\mathbb{R}^{d}$ respectively. 


\subsection{Fuzzy random variables}

A very interesting idea that describes the construction of fuzzy-valued probabilities by using fuzzy random variables is presented in the book [30] by Möller and Beer (compare Section 4.3). They use a definition (Definition 4.1 below) of fuzzy random variables by Wang and Zhang [44] that turns out amongst others to be equivalent to Kwakernaak's original definition.

Before stating their exact definition some abbreviations are helpful:

If $(\Omega, \mathcal{A}, \mathcal{P})$ is a probability space and $X^{\star}: \Omega \rightarrow \mathcal{F}_{c}^{1}$ is a fuzzy-valued function on $\Omega$, then the following notation will be used for every $\omega \in \Omega$ and every $\alpha \in(0,1]$ throughout the whole chapter:

$$
\begin{aligned}
& X_{\alpha}(\omega):=\left[X^{\star}(\omega)\right]_{\alpha}=\left\{x \in \mathbb{R}:\left(X^{\star}(\omega)\right)(x) \geq \alpha\right\} \\
& \underline{X}_{\alpha}(\omega):=\min \left(X_{\alpha}(\omega)\right), \quad \bar{X}_{\alpha}(\omega):=\max \left(X_{\alpha}(\omega)\right)
\end{aligned}
$$

It is clear by definition that the maximum and the minimum exist and that

$$
X_{\alpha}(\omega)=\left[\underline{X}_{\alpha}(\omega), \bar{X}_{\alpha}(\omega)\right] \quad \forall \alpha \in(0,1] \text { and } \forall \omega \in \Omega .
$$

Obviously $X_{\alpha}$ is an interval-valued function on $(\Omega, \mathcal{A}, \mathcal{P})$.

Definition 4.1 (Wang, Zhang [44])

Let $(\Omega, \mathcal{A}, \mathcal{P})$ be a probability space and $\mathcal{B}(\mathbb{R})$ denote the Borel subsets of $\mathbb{R}$. Then a function $X^{\star}: \Omega \rightarrow \mathcal{F}_{c}^{1}$ is called fuzzy random variable if

$$
\left\{\omega \in \Omega: X_{\alpha}(\omega) \cap B \neq \emptyset\right\} \in \mathcal{A}
$$

holds for every $B \in \mathcal{B}(\mathbb{R})$ and every $\alpha \in(0,1]$.

Remark: It is clear that Definition 4.1 includes the case of classical (realvalued) random variables on $(\Omega, \mathcal{A}, \mathcal{P})$.

The following two results (compare [44]) give equivalent forms of (4.2) and show how the measurability condition can be stated in terms of the upper and lower bounds $\underline{X}_{\alpha}$ and $\bar{X}_{\alpha}$.

Lemma 4.2 Let $(\Omega, \mathcal{A}, \mathcal{P})$ be a probability space and $\mathcal{B}(\mathbb{R})$ denote the Borel subsets of $\mathbb{R}$. Then a function $X^{\star}: \Omega \rightarrow \mathcal{F}_{c}^{1}$ is a fuzzy random variable if and only if

$$
\left\{\omega \in \Omega: X_{\alpha}(\omega) \subseteq B\right\} \in \mathcal{A}
$$

holds for every $B \in \mathcal{B}(\mathbb{R})$ and every $\alpha \in(0,1]$. 
Proof: The lemma is an immediate consequence of the following equality:

$$
\begin{aligned}
\left\{\omega \in \Omega: X_{\alpha}(\omega) \subseteq B\right\} & =\left\{\omega \in \Omega: X_{\alpha}(\omega) \cap B^{c}=\emptyset\right\} \\
& =\Omega \backslash\left\{\omega \in \Omega: X_{\alpha}(\omega) \cap B^{c} \neq \emptyset\right\} \\
& =\left\{\omega \in \Omega: X_{\alpha}(\omega) \cap B^{c} \neq \emptyset\right\}^{c}
\end{aligned}
$$

Theorem 4.3 Let $(\Omega, \mathcal{A}, \mathcal{P})$ be a probability space and $\mathcal{B}(\mathbb{R})$ denote the Borel subsets of $\mathbb{R}$. Then a function $X^{\star}: \Omega \rightarrow \mathcal{F}_{c}^{1}$ is a fuzzy random variable if and only if $\underline{X}_{\alpha}$ and $\bar{X}_{\alpha}$ defined according to (4.1) are classical (real-valued) random variables for every $\alpha \in(0,1]$, i.e.

$$
\begin{aligned}
& \underline{X}_{\alpha}^{-1}(B)=\left\{\omega \in \Omega: \underline{X}_{\alpha}(\omega) \in B\right\} \in \mathcal{A} \\
& \bar{X}_{\alpha}^{-1}(B)=\left\{\omega \in \Omega: \bar{X}_{\alpha}(\omega) \in B\right\} \in \mathcal{A}
\end{aligned}
$$

holds for every $B \in \mathcal{B}(\mathbb{R})$.

Proof: Suppose that $X^{\star}: \Omega \rightarrow \mathcal{F}_{c}^{1}$ is a fuzzy random variable and let $x \in \mathbb{R}$ be arbitrary, then it follows that

$$
\begin{aligned}
\underline{X}_{\alpha}^{-1}((-\infty, x]) & =\left\{\omega \in \Omega: \underline{X}_{\alpha}(\omega) \leq x\right\} \\
& =\left\{\omega \in \Omega: X_{\alpha}(\omega) \cap(-\infty, x] \neq \emptyset\right\} \in \mathcal{A} \quad \text { and } \\
\bar{X}_{\alpha}^{-1}([x, \infty)) & =\left\{\omega \in \Omega: \bar{X}_{\alpha}(\omega) \geq x\right\} \\
& =\left\{\omega \in \Omega: X_{\alpha}(\omega) \cap[x, \infty) \neq \emptyset\right\} \in \mathcal{A} .
\end{aligned}
$$

Since both systems $\mathfrak{E}_{1}=\{(-\infty, x]: x \in \mathbb{R}\}$ and $\mathfrak{E}_{2}=\{[x, \infty): x \in \mathbb{R}\}$ are generators of $\mathcal{B}(\mathbb{R})$, it follows immediately that $\underline{X}_{\alpha}$ and $\bar{X}_{\alpha}$ are measurable and therefore random variables for every $\alpha \in(0,1]$. This proves one half of the theorem.

In order to prove the other direction suppose that $\underline{X}_{\alpha}$ and $\bar{X}_{\alpha}$ are measurable and let $B \in \mathcal{B}(\mathbb{R})$ be arbitrary. For every $x \in B$ let $C(x)$ denote the (maximal) connected component of $B$ that contains $x$. It is clear that $C(x)$ is a single point or an interval with non-empty interior and that all connected components of $B$ are pairwise disjoint.

If $B_{1}$ denotes the set of all $x \in B$ such that $C(x)$ is a single point, then $B \backslash B_{1}$ can be written in the form

$$
B \backslash B_{1}=\bigcup_{i=1}^{N}\left\langle a_{i}, b_{i}\right\rangle,
$$

where $\left\langle a_{i}, b_{i}\right\rangle$ for every $i \in\{1, \cdots, N\}$ denotes a closed, open or semiopen interval with non-empty interior, $\left(\left\langle a_{i}, b_{i}\right\rangle\right)_{i=1}^{N}$ is a pairwise disjoint family, and 
$N \in \mathbb{N} \cup\{\infty\}$ (it is clear that there are at most countably many connected components with non-empty interior because of the separability of $\mathbb{R}$ ).

Using this decomposition and the fact that $X_{\alpha}(\omega)$ is a compact interval for every $\alpha \in(0,1]$ and every $\omega \in \Omega$ by definition it follows that

$$
\begin{aligned}
\left\{\omega \in \Omega: X_{\alpha}(\omega) \subseteq B\right\}= & \left\{\omega \in \Omega: X_{\alpha}(\omega) \subseteq B_{1} \cup \bigcup_{i=1}^{N}\left\langle a_{i}, b_{i}\right\rangle\right\} \\
= & \left\{\omega \in \Omega: X_{\alpha}(\omega) \subseteq B_{1} \text { and } \underline{X}_{\alpha}(\omega)=\bar{X}_{\alpha}(\omega)\right\} \cup \\
& \cup\left\{\omega \in \Omega: X_{\alpha}(\omega) \subseteq \bigcup_{i=1}^{N}\left\langle a_{i}, b_{i}\right\rangle\right\} \\
= & \left\{\omega \in \Omega: X_{\alpha}(\omega) \subseteq B_{1} \text { and } \underline{X}_{\alpha}(\omega)=\bar{X}_{\alpha}(\omega)\right\} \cup \\
& \cup \underbrace{\bigcup_{i=1}^{N}\left\{\omega \in \Omega: X_{\alpha}(\omega) \subseteq\left\langle a_{i}, b_{i}\right\rangle\right\}}_{A} \\
= & \underbrace{\left\{\omega \in \bigcup_{i=1}^{N}\left(\underline{X}_{\alpha}^{-1}\left(\left\langle a_{i}, \infty\right)\right) \cap \bar{X}_{\alpha}^{-1}\left(\left(-\infty, b_{i}\right\rangle\right)\right)\right.}_{C} .
\end{aligned}
$$

Since $\underline{X}_{\alpha}$ and $\bar{X}_{\alpha}$ are measurable it follows immediately that $A \in \mathcal{A}$. Furthermore $C$ is as (at most) countable union of measurable sets itself measurable, which implies that $C \in \mathcal{A}$. This shows that $\left\{\omega \in \Omega: X_{\alpha}(\omega) \subseteq B\right\}=A \cup C \in$ $\mathcal{A}$ for every $\alpha \in(0,1]$. Since $B \in \mathcal{B}(\mathbb{R})$ was arbitrary this completes the prove of the theorem.

Suppose for the moment that $(\Omega, \mathcal{A}, \mathcal{P})$ is a probability space and $X: \Omega \rightarrow \mathbb{R}$ is a random variable. It is well known (compare [13]) that the graph $\Gamma(X)$, defined by

$$
\Gamma(X)=\{(\omega, X(\omega)): \omega \in \Omega\} \subseteq \Omega \times \mathbb{R}
$$

fulfills $\Gamma(X) \in \mathcal{A} \otimes \mathcal{B}(\mathbb{R})$.

If $X^{\star}: \Omega \rightarrow \mathcal{F}_{c}^{1}$ is a fuzzy-valued function on $\Omega$ and the graph $\Gamma\left(X_{\alpha}\right)$ of $X_{\alpha}$ is for $\alpha \in(0,1]$ defined by

$$
\Gamma\left(X_{\alpha}\right)=\left\{(\omega, x): \omega \in \Omega \text { and } x \in X_{\alpha}(\omega)\right\} \subseteq \Omega \times \mathbb{R}
$$

then the measurability result mentioned above remains true if $X^{\star}$ is a fuzzy random variable.

This is the content of the next theorem. 
Theorem 4.4 Suppose $(\Omega, \mathcal{A}, \mathcal{P})$ is a probability space and $\mathcal{B}(\mathbb{R})$ denotes the Borel subsets of $\mathbb{R}$. If $X^{\star}: \Omega \rightarrow \mathcal{F}_{c}^{1}$ is a fuzzy random variable then $\Gamma\left(X_{\alpha}\right)$ defined according to (4.3) is measurable, i.e. $\Gamma\left(X_{\alpha}\right) \in \mathcal{A} \otimes \mathcal{B}(\mathbb{R})$ holds.

Proof: Define a function $F_{\alpha}: \Omega \times \mathbb{R} \longrightarrow \mathbb{R}$ for $\alpha \in(0,1]$ by

$$
F_{\alpha}(\omega, x)=x-\bar{X}_{\alpha}(\omega)
$$

The function $\Phi_{\alpha}: \Omega \times \mathbb{R} \longrightarrow \mathbb{R}^{2}$, defined by

$$
\Phi_{\alpha}(\omega, x)=\left(x, \bar{X}_{\alpha}(\omega)\right)
$$

is surely measurable since both coordinates are measurable and the function $M: \mathbb{R}^{2} \longrightarrow \mathbb{R}, M(x, y):=x-y$ is continuous and therefore Borel-measurable. Because of $M \circ \Phi_{\alpha}=F_{\alpha}$ it follows immediately that $F_{\alpha}$ is $\mathcal{A} \otimes \mathcal{B}(\mathbb{R})-\mathcal{B}(\mathbb{R})$ measurable. Consequently

$$
\Gamma_{-}\left(X_{\alpha}\right):=\left\{(\omega, x) \in \Omega \times \mathbb{R}: x \leq \bar{X}_{\alpha}(\omega)\right\}=F_{\alpha}^{-1}((-\infty, 0]) \in \mathcal{A} \otimes \mathcal{B}(\mathbb{R}) .
$$

In the same manner ist can easily be shown that

$$
\Gamma_{+}\left(X_{\alpha}\right):=\left\{(\omega, x) \in \Omega \times \mathbb{R}: x \geq \underline{X}_{\alpha}(\omega)\right\} \in \mathcal{A} \otimes \mathcal{B}(\mathbb{R}) .
$$

Finally because of $\Gamma_{+}\left(X_{\alpha}\right) \cap \Gamma_{-}\left(X_{\alpha}\right)=\Gamma\left(X_{\alpha}\right)$ it follows immediately that $\Gamma\left(X_{\alpha}\right) \in \mathcal{A} \otimes \mathcal{B}(\mathbb{R})$ for every $\alpha \in(0,1]$, which completes the proof.

Having Theorem 4.4 the question arises, whether the converse is true as well, i.e.:

Question 4.5 Given a fuzzy-valued function $X^{\star}: \Omega \rightarrow \mathcal{F}_{c}^{1}$ on a probability space $(\Omega, \mathcal{A}, \mathcal{P})$, does $\Gamma\left(X_{\alpha}\right) \in \mathcal{A} \otimes \mathcal{B}(\mathbb{R})$ for every $\alpha \in(0,1]$ imply that $X^{\star}$ is a fuzzy random variable?

Regarding classical random variables $X$ a complete answer is given (amongst others) in Bierlein [4]. Bierlein showed that a real-valued function $X$ defined on a complete measure space $(\Omega, \mathcal{A}, \mathcal{P})$ is measurable if and only if the graph $\Gamma(X)$ is the complement of a countable union of measurable rectangles and that this is equivalent to $\Gamma(X) \in \mathcal{A} \otimes \mathcal{B}(\mathbb{R})$. (For further generalizations of Bierlein's result compare for example [5], [25], [26]).

Within [4] a central lemma is proved using the theory of Choquet capacities, but this lemma can also be proved in a different (and more elementary) manner using the theory of Souslin sets. Therefore a short introduction into the theory of Souslin sets, presenting some main results that will be used to answer Question 4.5, is given in the Appendix.

In order to be able to apply these results one last step is necessary.

Remember the following definition: 
Definition 4.6 Let $\Omega$ be an arbitrary set, then a collection $\mathcal{R}$ of subsets of $\Omega$ is called $a \sigma-\delta$-ring if $\emptyset, \Omega \in \mathcal{R}$ and $\mathcal{R}$ is closed under countable intersections and countable unions.

Since an arbitrary intersection of $\sigma$ - $\delta$-rings is again a $\sigma-\delta$-ring it follows that for every family $\mathcal{E}$ of subsets of $\Omega$ there exists a minimal $\sigma-\delta$-ring that contains $\mathcal{E}$. This ring is called the $\sigma-\delta$-ring generated by $\mathcal{E}$ and will be denoted by $\mathcal{R}(\mathcal{E})$. In the same manner the $\sigma$-algebra generated by $\mathcal{E}$ will be denoted by $\mathcal{A}_{\sigma}(\mathcal{E})$.

Lemma 4.7 Suppose that $\mathcal{E}$ is a family of subsets of a given set $\Omega$. If $\mathcal{E}^{c}=$ $\left\{A^{c}: A \in \mathcal{E}\right\} \subseteq \mathcal{R}(\mathcal{E})$ then $\mathcal{R}(\mathcal{E})=\mathcal{A}_{\sigma}(\mathcal{E})$ holds.

Proof: The Theorem can be proved easily by following the ideas of the proof of Theorem 6.6: Since every $\sigma$-algebra obviously is a $\sigma-\delta$-ring it follows that $\mathcal{R}(\mathcal{E}) \subseteq \mathcal{A}_{\sigma}(\mathcal{E})$. Defining $\mathcal{R}^{\prime}:=\left\{A \in \mathcal{R}(\mathcal{E}): A^{c} \in \mathcal{R}(\mathcal{E})\right\} \subseteq \mathcal{R}(\mathcal{E})$ it follows that $\mathcal{R}^{\prime}$ is closed under complementation and countable unions.

Therefore $\mathcal{R}^{\prime}$ is a $\sigma$-algebra. Using the fact that $\mathcal{E}^{c} \subseteq \mathcal{R}(\mathcal{E})$ shows that $\mathcal{R}^{\prime}$ is a $\sigma$-algebra containing $\mathcal{E}$. Consequently

$$
\mathcal{E} \subseteq \mathcal{R}^{\prime} \subseteq \mathcal{R}(\mathcal{E}) \subseteq \mathcal{A}_{\sigma}(\mathcal{E}) \subseteq \mathcal{R}^{\prime}
$$

which shows that $\mathcal{R}^{\prime}=\mathcal{R}(\mathcal{E})=\mathcal{A}_{\sigma}(\mathcal{E})$. This completes the proof.

Having this the following lemma can be proved:

Lemma 4.8 Suppose that $\mathcal{A}$ is a $\sigma$-algebra in $\Omega$ and denote by $\mathcal{B}(\mathbb{R})$ the Borel $\sigma$-algebra in $\mathbb{R}$. Define $\mathfrak{J}:=\{[a, b] \subseteq \mathbb{R}:-\infty<a \leq b<\infty\} \cup \emptyset$ and

$$
\begin{aligned}
\mathfrak{h} & :=\{A \times B: A \in \mathcal{A}, B \in \mathcal{B}(\mathbb{R})\} \\
\tilde{\mathfrak{h}} & :=\{A \times J: A \in \mathcal{A} \text { and } J \in \mathfrak{J}\} .
\end{aligned}
$$

Then it follows that $\mathcal{A} \otimes \mathcal{B}(\mathbb{R}) \subseteq \mathcal{G}_{\text {sous }}(\mathfrak{h})=\mathcal{G}_{\text {sous }}(\tilde{\mathfrak{h}})$.

Proof: It is clear that $\mathfrak{h}$ is a generator of the product- $\sigma$-algebra $\mathcal{A} \otimes \mathcal{B}(\mathbb{R})$ and it is easy to see that $\tilde{\mathfrak{h}}$ is a generator too. Therefore

$$
\mathcal{A}_{\sigma}(\mathfrak{h})=\mathcal{A}_{\sigma}(\tilde{\mathfrak{h}})=\mathcal{A} \otimes \mathcal{B}(\mathbb{R})
$$

Since $(A \times[a, b])^{c}=\left(A^{c} \times \mathbb{R}\right) \cup\left(A \times[a, b]^{c}\right) \in \mathcal{R}(\tilde{\mathfrak{h}})$ for every $(A \times[a, b]) \in \tilde{\mathfrak{h}}$ it follows immediately that $\tilde{\mathfrak{h}}^{c} \subseteq \mathcal{R}(\tilde{\mathfrak{h}})$. Consequently

$$
\mathcal{R}(\tilde{\mathfrak{h}})=\mathcal{A}_{\sigma}(\tilde{\mathfrak{h}})=\mathcal{A} \otimes \mathcal{B}(\mathbb{R})
$$

according to Lemma 4.7 . Furthermore since $\mathcal{G}_{\text {sous }}(\tilde{\mathfrak{h}})$ is a $\sigma$ - $\delta$-ring containing $\tilde{\mathfrak{h}}$, by Theorem 6.5 it follows that

$$
\mathfrak{h} \subseteq \mathcal{A} \otimes \mathcal{B}(\mathbb{R})=\mathcal{A}_{\sigma}(\tilde{\mathfrak{h}})=\mathcal{R}(\tilde{\mathfrak{h}}) \subseteq \mathcal{G}_{\text {sous }}(\tilde{\mathfrak{h}}) \subseteq \mathcal{G}_{\text {sous }}(\mathfrak{h})
$$


Using the idempotence of the Souslin Operation (Theorem 6.4) this proves that

$$
\mathcal{G}_{\text {sous }}(\mathfrak{h}) \subseteq \mathcal{G}_{\text {sous }}(\mathcal{A} \otimes \mathcal{B}(\mathbb{R})) \subseteq \mathcal{G}_{\text {sous }}(\tilde{\mathfrak{h}}) \subseteq \mathcal{G}_{\text {sous }}(\mathfrak{h})
$$

Therefore $\mathcal{A} \otimes \mathcal{B}(\mathbb{R}) \subseteq \mathcal{G}_{\text {sous }}(\mathfrak{h})=\mathcal{G}_{\text {sous }}(\tilde{\mathfrak{h}})$ holds.

As usual denote by $\pi_{1}: \Omega \times \mathbb{R} \longrightarrow \Omega$ the projection of $\Omega \times \mathbb{R}$ on $\Omega$, defined by $\pi_{1}(x, y):=x$ for all $(x, y) \in \Omega \times \mathbb{R}$. Furthermore for $A \subseteq \Omega \times \mathbb{R}$ the projection $\pi_{1}(A)$ is defined by $\pi_{1}(A):=\{x \in \Omega: \exists y \in \mathbb{R}$ with $(x, y) \in A\}$.

Theorem 4.9 Suppose that the conditions of Lemma 4.8 hold. Then for arbitrary $C \in \mathcal{G}_{\text {sous }}(\mathfrak{h})$ it follows that $\pi_{1}(C) \in \mathcal{G}_{\text {sous }}(\mathcal{A})$.

Proof: If $C \in \mathcal{G}_{\text {sous }}(\mathfrak{h})=\mathcal{G}_{\text {sous }}(\tilde{\mathfrak{h}})$ is an arbitrary set then there exists a tree $\left\{R_{s}=A_{s} \times I_{s} \in \tilde{\mathfrak{h}}: s \in \mathbb{N}^{<\mathbb{N}}\right\}$ in $\tilde{\mathfrak{h}}$ such that

$$
C=\bigcup_{\alpha \in \mathbb{N}^{N}} \bigcap_{n=1}^{\infty} R_{\alpha \mid n}
$$

Without loss of generality assume both that the tree $\left\{R_{s}\right\}$ is regular (which is possible since the family $\mathfrak{J}$ is closed under intersection) and that $A_{s}=\emptyset$ and $I_{s}=\emptyset$ if $R_{s}=\emptyset$. Consequently both $\left\{A_{s}: s \in \mathbb{N}<\mathbb{N}\right\}$ and $\left\{I_{s}: s \in \mathbb{N}<\mathbb{N}\right\}$ are regular trees. Define for arbitrary $\sigma \in \mathbb{N}^{\mathbb{N}}$

$$
R^{\sigma}:=\bigcap_{n=1}^{\infty} R_{\sigma \mid n}, \quad A^{\sigma}:=\bigcap_{n=1}^{\infty} A_{\sigma \mid n} \quad \text { and } \quad I^{\sigma}:=\bigcap_{n=1}^{\infty} I_{\sigma \mid n} .
$$

Obviously $R^{\sigma}=A^{\sigma} \times I^{\sigma}$ holds for every $\sigma \in \mathbb{N}^{\mathbb{N}}$. Therefore if $R^{\sigma}=\emptyset$ it follows immediately that at least one of $A^{\sigma}, I^{\sigma}$ is empty.

If $I^{\sigma}=\emptyset$ then there exists a $n_{0} \in \mathbb{N}$ such that $I_{\sigma \mid n}=\emptyset$ for every $n \geq n_{0}$ $\left(I_{\sigma \mid n} \neq \emptyset\right.$ for every $n \in \mathbb{N}$ can not hold since this would imply that $I^{\sigma} \neq \emptyset$ by the intersection property). Therefore $R_{\sigma \mid n_{0}}=\emptyset$ holds, which gives $A_{\sigma \mid n_{0}}=\emptyset$ by assumption and shows that $A^{\sigma}=\emptyset$. This proves that $R^{\sigma}=\emptyset$ implies $A^{\sigma}=\emptyset$. Having this it follows immediately that $\pi_{1}\left(R^{\sigma}\right)=A^{\sigma}$ for every $\sigma \in \mathbb{N}^{\mathbb{N}}$, which shows that

$$
\pi_{1}(C)=\bigcup_{\sigma \in \mathbb{N}^{\mathbb{N}}} \pi_{1}\left(R^{\sigma}\right)=\bigcup_{\sigma \in \mathbb{N}^{\mathbb{N}}} A^{\sigma}=\bigcup_{\sigma \in \mathbb{N}^{\mathbb{N}}} \bigcap_{n=1}^{\infty} A_{\sigma \mid n} \in \mathcal{G}_{\text {sous }}(\mathcal{A}),
$$

and completes the proof of the theorem.

Theorem 4.10 Suppose that $(\Omega, \mathcal{A}, \mu)$ is a complete $\sigma$-finite measure space and denote by $\mathcal{B}(\mathbb{R})$ the Borel $\sigma$-algebra in $\mathbb{R}$.

Then for every set $C \in \mathcal{A} \otimes \mathcal{B}(\mathbb{R})$ the projection $\pi_{1}(C)$ of $C$ on $\Omega$ fulfills $\pi_{1}(C) \in \mathcal{G}_{\text {sous }}(\mathcal{A}) \subseteq \mathcal{A}$. 
Proof: Since $\mathcal{A} \otimes \mathcal{B}(\mathbb{R}) \subseteq \mathcal{G}_{\text {sous }}(\tilde{\mathfrak{h}})$ by Lemma 4.8 , and $\pi_{1}(C) \in \mathcal{G}_{\text {sous }}(\mathcal{A})$ for every $C \in \mathcal{G}_{\text {sous }}(\tilde{\mathfrak{h}})$ by Theorem 4.9 it follows that $\pi_{1}(C) \in \mathcal{G}_{\text {sous }}(\mathcal{A})$ for every $C \in \mathcal{A} \otimes \mathcal{B}(\mathbb{R})$. The fact that $\mathcal{G}_{\text {sous }}(\mathcal{A})=\mathcal{A}$ (Theorem 6.10) completes the proof.

Finally the complete answer to the Question 4.5 is given in the next theorem, which is the converse of Theorem 4.4.

Theorem 4.11 Let $(\Omega, \mathcal{A}, \mathcal{P})$ be a complete probability space and $\mathcal{B}(\mathbb{R})$ denote the Borel subsets of $\mathbb{R}$. If for $X^{\star}: \Omega \rightarrow \mathcal{F}_{c}^{1}$ the graph $\Gamma\left(X_{\alpha}\right)$ defined according to (4.3) is measurable for every $\alpha \in(0,1]$ then $X^{\star}$ is a fuzzy random variable.

Proof: According to Theorem 4.3 the fact that $X^{\star}$ is a fuzzy random variable is equivalent to the condition that $\underline{X}_{\alpha}: \Omega \longrightarrow \mathbb{R}$ and $\bar{X}_{\alpha}: \Omega \longrightarrow \mathbb{R}$ are classical random variables. Suppose that $\Gamma\left(X_{\alpha}\right) \in \mathcal{A} \otimes \mathcal{B}(\mathbb{R})$ holds for every $\alpha \in(0,1]$ and let $x \in \mathbb{R}$ be arbitrary, then it follows that

$$
\begin{aligned}
\underline{X}_{\alpha}^{-1}((-\infty, x]) & =\left\{\omega \in \Omega: \underline{X}_{\alpha}(\omega) \leq x\right\} \\
& =\left\{\omega \in \Omega: \exists y \in X_{\alpha}(\omega) \text { such that } y \leq x\right\} \\
& =\pi_{1}\left(\left\{(\omega, y) \in \Omega \times \mathbb{R}: y \in X_{\alpha}(\omega) \text { and } y \leq x\right\}\right) \\
& =\pi_{1}\left(\Gamma\left(X_{\alpha}\right) \cap(\Omega \times(-\infty, x])\right) \in \mathcal{A}, \\
\bar{X}_{\alpha}^{-1}([x, \infty)) & =\left\{\omega \in \Omega: \bar{X}_{\alpha}(\omega) \geq x\right\} \\
& =\left\{\omega \in \Omega: \exists y \in X_{\alpha}(\omega) \text { such that } y \geq x\right\} \\
& =\pi_{1}\left(\left\{(\omega, y) \in \Omega \times \mathbb{R}: y \in X_{\alpha}(\omega) \text { and } y \geq x\right\}\right) \\
& =\pi_{1}\left(\Gamma\left(X_{\alpha}\right) \cap(\Omega \times[x, \infty))\right) \in \mathcal{A} .
\end{aligned}
$$

Since both systems $\mathfrak{E}_{1}=\{(-\infty, x]: x \in \mathbb{R}\}$ and $\mathfrak{E}_{2}=\{[x, \infty): x \in \mathbb{R}\}$ are generators of $\mathcal{B}(\mathbb{R})$, it follows that $\underline{X}_{\alpha}$ and $\bar{X}_{\alpha}$ are measurable and therefore random variables for every $\alpha \in(0,1]$.

Theorem 4.11 is the key for proving other equivalent measurability conditions for a fuzzy-valued mapping $X^{\star}: \Omega \rightarrow \mathcal{F}_{c}^{1}$ on a complete probability space $(\Omega, \mathcal{A}, \mathcal{P})$, which are summarized in the next theorem (compare [7] and $[17]):$

Theorem 4.12 Let $(\Omega, \mathcal{A}, \mathcal{P})$ be a complete probability space and $\mathcal{B}(\mathbb{R})$ denote the Borel subsets of $\mathbb{R}$. Then for a fuzzy-valued mapping $X^{\star}: \Omega \rightarrow \mathcal{F}_{c}^{1}$ the following nine conditions are equivalent: 
1. $\left\{\omega \in \Omega: X_{\alpha}(\omega) \cap B \neq \emptyset\right\} \in \mathcal{A}$ holds for every $B \in \mathcal{B}(\mathbb{R})$ and every $\alpha \in(0,1]$ (i.e. $X^{\star}$ is a fuzzy random variable).

2. $\left\{\omega \in \Omega: X_{\alpha}(\omega) \subseteq B\right\} \in \mathcal{A}$ holds for every $B \in \mathcal{B}(\mathbb{R})$ and every $\alpha \in(0,1]$.

3. $\left\{\omega \in \Omega: X_{\alpha}(\omega) \cap C \neq \emptyset\right\} \in \mathcal{A}$ holds for every closed set $C \subseteq \mathbb{R}$ and every $\alpha \in(0,1]$.

4. $\left\{\omega \in \Omega: X_{\alpha}(\omega) \cap K \neq \emptyset\right\} \in \mathcal{A}$ holds for every compact set $K \subseteq \mathbb{R}$ and every $\alpha \in(0,1]$ (i.e. $X_{\alpha}$ is a random closed set for every $\alpha \in(0,1]$ ).

5. $\left\{\omega \in \Omega: X_{\alpha}(\omega) \cap G \neq \emptyset\right\} \in \mathcal{A}$ holds for every open set $G \subseteq \mathbb{R}$ and every $\alpha \in(0,1]$ (i.e. $X_{\alpha}$ is Effros measurable for every $\alpha \in(0,1]$, compare for instance [7], [29]).

6. $\omega \mapsto d\left(x, X_{\alpha}(\omega)\right):=\inf _{y \in X_{\alpha}(\omega)}|x-y|$ is a Borel-measurable function for every fixed $x \in \mathbb{R}$ and every $\alpha \in(0,1]$.

7. $\underline{X}_{\alpha}$ and $\bar{X}_{\alpha}$ defined according to (4.1) are classical (real-valued) random variables for every $\alpha \in(0,1]$.

8. $\Gamma\left(X_{\alpha}\right)$ defined according to (4.3) is measurable for every $\alpha \in(0,1]$, i.e. $\Gamma\left(X_{\alpha}\right) \in \mathcal{A} \otimes \mathcal{B}(\mathbb{R})$ holds for every $\alpha \in(0,1]$.

9. For every $\alpha \in(0,1]$ there exists a sequence $\left(f_{n}\right)_{n \in \mathbb{N}}$ of measurable selections of $X_{\alpha}$ such that the following equality holds for every $\omega \in \Omega$ :

$\overline{\left\{f_{n}(\omega): n \in \mathbb{N}\right\}}=X_{\alpha}(\omega)$ (Castaing representation, compare [7], [29])

Proof: It has already been proved in this section that the points one, two, seven and eight are equivalent. Furthermore it is trivial that point one implies implies point three and that point three implies point four.

Consequently it suffices to prove that

- point four implies point five (a)

- point five is equivalent to point six (b)

- point five implies to point seven (c)

- point seven implies point nine (d)

- point nine implies point five (e). 
For every $\alpha \in(0,1]$ and every set $A \subseteq \mathbb{R}$ define

$$
X_{\alpha}^{\sim 1}(A):=\left\{\omega \in \Omega: X_{\alpha}(\omega) \cap A \neq \emptyset\right\} .
$$

Then it follows immediately that for every family $\left(A_{i}\right)_{i \in I}$ ( $I$ an arbitrary index set)

$$
X_{\alpha}^{\sim 1}\left(\bigcup_{i \in I} A_{i}\right)=\bigcup_{i \in I} X_{\alpha}^{\sim 1}\left(A_{i}\right)
$$

holds.

(a) It is well-known that the family $\mathfrak{B}$ consisting of all open balls $B(x, r)$ with $x \in \mathbb{Q}$ and $r \in \mathbb{Q}^{+}$is a countable base for the Euclidean topology and that every ball $B(x, r)$ is precompact (i.e. the closure $\overline{B(x, r)}$ is compact). Consequently for every open set $G$ the following representation holds:

$$
G=\bigcup_{\bar{B} \subseteq G, B \in \mathfrak{B}} B=\bigcup_{\bar{B} \subseteq G, B \in \mathfrak{B}} \bar{B}
$$

Assume that point four is fulfilled, then using (4.5) and the countability of $\mathfrak{B}$ yields

$$
X_{\alpha}^{\sim 1}(G)=X_{\alpha}^{\sim 1}\left(\bigcup_{\bar{B} \subseteq G, B \in \mathfrak{B}} \bar{B}\right)=\bigcup_{\bar{B} \subseteq G, B \in \mathfrak{B}} X_{\alpha}^{\sim 1}(\bar{B}) \in \mathcal{A},
$$

which completes the proof of (a), since $\alpha \in(0,1]$ was arbitrary.

(b) In order to prove the equivalence of point five and point six define for every $x \in \mathbb{R}$ and $\omega \in \Omega$ :

$$
g_{x}(\omega)=d\left(x, X_{\alpha}(\omega)\right):=\inf _{y \in X_{\alpha}(\omega)}|x-y|
$$

The desired equivalence is an immediate consequence of the following interrelation, which holds for every $x \in \mathbb{R}$ and $c>0$, and the fact that the countable base $\mathfrak{B}$ exactly consists of sets of the form $B(x, c)(B(x, c) \subseteq \mathbb{R}$ as common denotes the open ball with radius $c$ and center $x$ ):

$$
\begin{aligned}
X_{\alpha}^{\sim 1}(B(x, c)) & =\left\{\omega \in \Omega: X_{\alpha}(\omega) \cap B(x, c) \neq \emptyset\right\} \\
& =\left\{\omega \in \Omega: d\left(x, X_{\alpha}(\omega)<c\right\}\right. \\
& =g_{x}^{-1}((-\infty, c))
\end{aligned}
$$

(c) Suppose that $X^{\star}: \Omega \rightarrow \mathcal{F}_{c}^{1}$ fulfills point five, fix $\alpha \in(0,1]$ and let $x \in \mathbb{R}$ be arbitrary, then it follows that

$$
\begin{aligned}
\underline{X}_{\alpha}^{-1}((-\infty, x)) & =\left\{\omega \in \Omega: \underline{X}_{\alpha}(\omega)<x\right\} \\
& =\left\{\omega \in \Omega: X_{\alpha}(\omega) \cap(-\infty, x) \neq \emptyset\right\} \in \mathcal{A} \quad \text { and } \\
\bar{X}_{\alpha}^{-1}((x, \infty)) & =\left\{\omega \in \Omega: \bar{X}_{\alpha}(\omega)>x\right\} \\
& =\left\{\omega \in \Omega: X_{\alpha}(\omega) \cap(x, \infty) \neq \emptyset\right\} \in \mathcal{A} .
\end{aligned}
$$


Since both systems $\mathfrak{E}_{1}^{\prime}=\{(-\infty, x): x \in \mathbb{R}\}$ and $\mathfrak{E}_{2}^{\prime}=\{(x, \infty): x \in \mathbb{R}\}$ are generators of $\mathcal{B}(\mathbb{R})$, it follows immediately that $\underline{X}_{\alpha}$ and $\bar{X}_{\alpha}$ are measurable and therefore random variables for every $\alpha \in(0,1]$. Since $\alpha \in(0,1]$ was arbitrary step (c) is proved, which (using the equivalence of point seven and point one) completes the proof that alle the points one to eight are equivalent. It remains to prove that the Castaing representation (point nine) also is equivalent.

(d) Given the measurablility of $\underline{X}_{\alpha}$ and $\bar{X}_{\alpha}$ for every $\alpha \in(0,1]$, the Castaing representation is easy to prove:

Denote by $\mathbb{Q}_{[0,1]}=\left\{q_{1}, q_{2}, \cdots\right\}$ the countable set of all rational numbers in the interval $[0,1]$. Fix an arbitrary $\alpha \in(0,1]$ and for every $n \in \mathbb{N}$ define a function $f_{n}$ by

$$
f_{n}(\omega):=q_{n} \underline{X}_{\alpha}(\omega)+\left(1-q_{n}\right) \bar{X}_{\alpha}(\omega) \quad \forall \omega \in \Omega .
$$

As convex combination of measurable functions every function $f_{n}$ is measurable and a selection of $X_{\alpha}$ too. Furthermore because of the fact that $\mathbb{Q}_{[0,1]}$ is dense in $[0,1]$ the desired property $\overline{\left\{f_{n}(\omega): n \in \mathbb{N}\right\}}=X_{\alpha}(\omega)$ obviously is fulfilled. Since $\alpha$ was arbitrary this proves the implication (d).

(e) Finally suppose that for every fixed $\alpha \in(0,1]$ there exists a sequence of measurable selections $\left(f_{n}\right)_{n \in \mathbb{N}}$ of $X_{\alpha}$ such that

$$
\overline{\left\{f_{n}(\omega): n \in \mathbb{N}\right\}}=X_{\alpha}(\omega)
$$

holds for all $\omega \in \Omega$. If $G$ is an arbitrary open subset of $\mathbb{R}$ then

$$
\begin{aligned}
X_{\alpha}^{-1}(G) & =\left\{\omega \in \Omega: X_{\alpha}(\omega) \cap G \neq \emptyset\right\} \\
& =\left\{\omega \in \Omega: \overline{\left\{f_{n}(\omega): n \in \mathbb{N}\right\}} \cap G \neq \emptyset\right\} \\
& =\left\{\omega \in \Omega:\left\{f_{n}(\omega): n \in \mathbb{N}\right\} \cap G \neq \emptyset\right\} \\
& =\bigcup_{n=1}^{\infty}\left\{\omega: f_{n}(\omega) \in G\right\}=\bigcup_{n=1}^{\infty} f_{n}^{-1}(G) \in \mathcal{A}
\end{aligned}
$$

completes the proof.

Since by definition for every fuzzy-valued mapping $X^{\star}: \Omega \rightarrow \mathcal{F}_{c}^{1}$ the $\alpha$-cut mappings $X_{\alpha}$ (for every $\left.\alpha \in(0,1]\right)$ are $\mathcal{K}_{c}^{1}$-valued it is furthermore natural to analyze the interrelation of the measurability condition (4.2) and the measurability of the $\alpha$-cut mappings $X_{\alpha}$ with respect to the Borel $\sigma$-algebra $\mathcal{B}\left(\left(\mathcal{K}_{c}^{1}, \delta_{H}\right)\right)$ in $\mathcal{K}_{c}^{1}$ generated by the Hausdorff metric. Applying Theorem 1.20 the following result can be proved (compare [29]) - since it is a supplement to Theorem 4.12 the enumeration of Theorem 4.12 is carried on: 
Theorem 4.13 Suppose that $(\Omega, \mathcal{A}, \mathcal{P})$ is a complete probability space and let $\mathcal{B}\left(\left(\mathcal{K}_{c}^{1}, \delta_{H}\right)\right)$ denote the Borel $\sigma$-algebra in $\mathcal{K}_{c}^{1}$ generated by the Hausdorff metric $\delta_{H}$. Then the following conditions are equivalent for a fuzzy-valued mapping $X^{\star}: \Omega \rightarrow \mathcal{F}_{c}^{1}$ :

1. $X^{\star}$ is a fuzzy random variable according to Definition 4.1 .

10. For every $\alpha \in(0,1]$ the $\alpha$-cut mapping $X_{\alpha}: \Omega \rightarrow \mathcal{K}_{c}^{1}$ is measurable with respect to $\mathcal{B}\left(\left(\mathcal{K}_{c}^{1}, \delta_{H}\right)\right)$.

Proof: According to Theorem 4.12 a fuzzy-valued mapping $X^{\star}: \Omega \rightarrow \mathcal{F}_{c}^{1}$ is a fuzzy random variable if and only if $\left\{\omega \in \Omega: X_{\alpha}(\omega) \cap G \neq \emptyset\right\} \in \mathcal{A}$ holds for every open set $G \subseteq \mathbb{R}$. Moreover since the family

$$
\mathscr{E}_{2, c}^{1}=\left\{\mathcal{K}_{G}=\left\{K \in \mathcal{K}_{c}^{1}: K \cap G \neq \emptyset\right\}: G \subseteq \mathbb{R}, G \text { open }\right\}
$$

according to Proposition 1.21 generates $\mathcal{B}\left(\left(\mathcal{K}_{c}^{1}, \delta_{H}\right)\right)$ the desired equivalence follows immediately since obviously for every open set $G \subseteq \mathbb{R}$ and every $\alpha \in(0,1]$ the equality

$$
X_{\alpha}^{-1}\left(\mathcal{K}_{G}\right)=\left\{\omega \in \Omega: X_{\alpha}(\omega) \cap G \neq \emptyset\right\}
$$

holds.

In case that $X_{1}(\omega)$ does not only consist of one point for $\mathcal{P}$-almost every $\omega \in \Omega$, another equivalent easy manageable notion of measurability can be stated (the enumeration is continued once more), compare again [29].

Theorem 4.14 Suppose that $(\Omega, \mathcal{A}, \mathcal{P})$ is a complete probability space and that $X^{\star}: \Omega \rightarrow \mathcal{F}_{c}^{1}$ is a fuzzy-valued mapping on $\Omega$ such that $\underline{X}_{1}(\omega)<\bar{X}_{1}(\omega)$ holds for $\mathcal{P}$-almost every $\omega \in \Omega$. Then the following conditions are equivalent:

1. $X^{\star}$ is a fuzzy random variable according to Definition 4.1 .

11. $\left\{\omega \in \Omega: x \in X_{\alpha}(\omega)\right\} \in \mathcal{A}$ holds for every $\alpha \in(0,1]$ and every $x \in \mathbb{R}$.

Proof: One part of the equivalence is trivial:

If $X^{\star}$ is a fuzzy random variable then $\left\{\omega \in \Omega: x \in X_{\alpha}(\omega)\right\} \in \mathcal{A}$ surely holds since $\{x\}$ is closed and $x \in X_{\alpha}(\omega)$ is equivalent to $X_{\alpha}(\omega) \cap\{x\} \neq \emptyset$ for every $x \in \mathbb{R}$ and $\alpha \in(0,1]$.

Because of the fact that by assumption $\underline{X}_{1}(\omega)<\bar{X}_{1}(\omega)$ holds for $\mathcal{P}$-almost every $\omega \in \Omega$ there exists a set $N \in \mathcal{A}$ with $\mathcal{P}(N)=0$, such that for all $\omega \in N^{c} \operatorname{int}\left(X_{\alpha}(\omega)\right) \neq \emptyset$ is fulfilled $(\operatorname{int}(A)$ denotes the topological interior of $A$, i.e. the set of all inner points of $A$, and $\operatorname{cls}(A)$ denotes the closure of $A$, 
i.e. the intersection of all closed sets containing $A$, for every set $A$ ).

Suppose now that $G$ is open, $\alpha \in(0,1]$, and that condition eleven is fulfilled. Obviously the following equality holds:

$$
\begin{aligned}
\left\{\omega \in \Omega: X_{\alpha}(\omega) \cap G \neq \emptyset\right\} & =\overbrace{\left\{\omega \in N: X_{\alpha}(\omega) \cap G \neq \emptyset\right\}}^{=: A_{1}} U \\
& \cup \underbrace{\left\{\omega \in N^{c}: X_{\alpha}(\omega) \cap G \neq \emptyset\right\}}_{=: A_{2}}
\end{aligned}
$$

As a subset of the set $N$ of probability zero the set $A_{1}$ itself is measurable, i.e. $A_{1} \in \mathcal{A}$. Consequently it suffices to prove that $A_{2} \in \mathcal{A}$ holds, which can be done as follows:

$$
\begin{aligned}
A_{2}=\left\{\omega \in N^{c}: X_{\alpha}(\omega) \cap G \neq \emptyset\right\} & =\left\{\omega \in N^{c}: \operatorname{int}\left(X_{\alpha}(\omega)\right) \cap G \neq \emptyset\right\} \\
& =\left\{\omega \in N^{c}: \operatorname{int}\left(X_{\alpha}(\omega)\right) \cap G \cap \mathbb{Q} \neq \emptyset\right\} \\
& =N^{c} \cap \bigcup_{x \in \mathbb{Q} \cap G}\left\{\omega \in \Omega: x \in X_{\alpha}(\omega)\right\} \in \mathcal{A}
\end{aligned}
$$

This completes the proof.

Section 4.1 will be rounded off by a result which basically says that if a fuzzy-valued function $X^{\star}: \Omega \rightarrow \mathcal{F}_{c, c}^{1}$ is measurable with respect to the $\sigma$ algebra $\mathcal{B}\left(\left(\mathcal{F}_{c, c}^{1}, \delta_{H, \infty}^{\star}\right)\right)$ generated by the metric $\delta_{H, \infty}^{\star}$ (compare Section 1.5), then $X^{\star}$ is a fuzzy random variable.

Theorem 4.15 Suppose that $(\Omega, \mathcal{A}, \mathcal{P})$ is a complete probability space and that $X^{\star}: \Omega \rightarrow \mathcal{F}_{c, c}^{1}$ is a fuzzy-valued mapping on $\Omega$. Furthermore denote by $\mathcal{B}\left(\left(\mathcal{F}_{c, c}^{1}, \delta_{H, \infty}^{\star}\right)\right)$ the $\sigma$-algebra generated by the metric $\delta_{H, \infty}^{\star}$ in $\mathcal{F}_{c, c}^{1}$.

If $X^{\star}$ is measurable with respect to $\mathcal{B}\left(\left(\mathcal{F}_{c, c}^{1}, \delta_{H, \infty}^{\star}\right)\right)$ then $X^{\star}$ is a fuzzy random variable according to Definition 4.1.

Proof: For every $\alpha \in(0,1]$ define a mapping $\Pi_{\alpha}: \mathcal{F}_{c, c}^{1} \rightarrow \mathcal{K}_{c}^{1} \subseteq \mathcal{K}^{1}$ by $\Pi_{\alpha}\left(\xi^{\star}\right):=\left[\xi^{\star}\right]_{\alpha}$ for every $\xi^{\star} \in \mathcal{F}_{c, c}^{1}$. It follows immediately from the construction of $\delta_{H, \infty}^{\star}$ that $\Pi_{\alpha}$ is Lipschitz continuous with Lipschitz constant 1 (in fact $\Pi_{\alpha}$ is a projection). Consequently $\Pi_{\alpha}$ is $\mathcal{B}\left(\left(\mathcal{F}_{c, c}^{1}, \delta_{H, \infty}^{\star}\right)\right)-\mathcal{B}\left(\left(\mathcal{K}_{c}^{1}, \delta_{H}\right)\right.$ )measurable. Since the composition of two measurable mappings is itself measurable the fact that

$$
X_{\alpha}(\omega)=\Pi_{\alpha} \circ X^{\star}(\omega)
$$

holds for every $\omega \in \Omega$, together with Theorem 4.13 completes the proof of the theorem. 


\subsection{Fuzzy random vectors}

As already mentioned fuzzy random vectors are the multi-dimensional analogy of fuzzy random variables.

If $(\Omega, \mathcal{A}, \mathcal{P})$ is a probability space and $X^{\star}: \Omega \rightarrow \mathcal{F}_{c}^{d}(d \geq 2)$ is a fuzzyvector-valued function on $\Omega$, then the following notation (analogous to fuzzy random variables) will be used for every $\omega \in \Omega$ and every $\alpha \in(0,1]$ :

$$
X_{\alpha}(\omega):=\left[X^{\star}(\omega)\right]_{\alpha}=\left\{x \in \mathbb{R}^{d}:\left(X^{\star}(\omega)\right)(x) \geq \alpha\right\}
$$

Furthermore the graph $\Gamma\left(X_{\alpha}\right)$ is for every $\alpha \in(0,1]$ defined by

$$
\Gamma\left(X_{\alpha}\right)=\left\{(\omega, x): \omega \in \Omega \text { and } x \in X_{\alpha}(\omega)\right\} \subseteq \Omega \times \mathbb{R}^{d} .
$$

Definition 4.16 Let $(\Omega, \mathcal{A}, \mathcal{P})$ be a probability space and $\mathcal{B}\left(\mathbb{R}^{d}\right)$ denote the Borel subsets of $\mathbb{R}^{d}$. Then a function $X^{\star}: \Omega \rightarrow \mathcal{F}_{c}^{d}$ is called (d-dimensional) fuzzy random vector if

$$
\left\{\omega \in \Omega: X_{\alpha}(\omega) \cap B \neq \emptyset\right\} \in \mathcal{A}
$$

holds for every $B \in \mathcal{B}\left(\mathbb{R}^{d}\right)$ and every $\alpha \in(0,1]$.

As for fuzzy random variables the above definition of fuzzy random vectors is equivalent to various other conditions. Looking back at the proofs of the results in Section 4.1, especially that of Theorem 4.12, it may come as no suprise that similar results also hold for fuzzy random vectors since $\mathbb{R}$ and $\mathbb{R}^{d}$ are very similar from the topological point of view - both are $\sigma$-compact Polish spaces. In fact the following theorem, taken from [7] and [29] with some small modifications, holds:

Theorem 4.17 Let $(\Omega, \mathcal{A}, \mathcal{P})$ be a complete probability space and $\mathcal{B}\left(\mathbb{R}^{d}\right)$ denote the Borel subsets of $\mathbb{R}^{d}$. Then for a fuzzy vector-valued mapping $X^{\star}: \Omega \rightarrow \mathcal{F}_{c}^{d}$ the following conditions are equivalent:

$1^{d} .\left\{\omega \in \Omega: X_{\alpha}(\omega) \cap B \neq \emptyset\right\} \in \mathcal{A}$ holds for every $B \in \mathcal{B}\left(\mathbb{R}^{d}\right)$ and every $\alpha \in(0,1]$ (i.e. $X^{\star}$ is a d-dimensional fuzzy random vector).

$\mathscr{2}^{d} .\left\{\omega \in \Omega: X_{\alpha}(\omega) \subseteq B\right\} \in \mathcal{A}$ holds for every $B \in \mathcal{B}\left(\mathbb{R}^{d}\right)$ and every $\alpha \in(0,1]$.

$3^{d} .\left\{\omega \in \Omega: X_{\alpha}(\omega) \cap C \neq \emptyset\right\} \in \mathcal{A}$ holds for every closed set $C \subseteq \mathbb{R}^{d}$ and every $\alpha \in(0,1]$.

$4^{d} .\left\{\omega \in \Omega: X_{\alpha}(\omega) \cap K \neq \emptyset\right\} \in \mathcal{A}$ holds for every compact set $K \subseteq \mathbb{R}^{d}$ and every $\alpha \in(0,1]$ (i.e. $X_{\alpha}$ is a random closed set for every $\alpha \in(0,1]$ ). 
5. $\left\{\omega \in \Omega: X_{\alpha}(\omega) \cap G \neq \emptyset\right\} \in \mathcal{A}$ holds for every open set $G \subseteq \mathbb{R}^{d}$ and every $\alpha \in(0,1]$ (i.e. $X_{\alpha}$ is Effros measurable for every $\alpha \in(0,1]$ ).

$6^{d} . \omega \mapsto d\left(x, X_{\alpha}(\omega)\right):=\inf _{y \in X_{\alpha}(\omega)}\|x-y\|_{2}$ is a measurable function for every fixed $x \in \mathbb{R}$ and every $\alpha \in(0,1]$.

$7^{d}$. $\Gamma\left(X_{\alpha}\right)$ defined according to (4.8) is measurable for every $\alpha \in(0,1]$, i.e. $\Gamma\left(X_{\alpha}\right) \in \mathcal{A} \otimes \mathcal{B}\left(\mathbb{R}^{d}\right)$ holds for every $\alpha \in(0,1]$.

$8^{d}$. For every $\alpha \in(0,1]$ there exists a sequence $\left(f_{n}\right)_{n \in \mathbb{N}}$ of measurable selections of $X_{\alpha}$ such that the following equality holds for every $\omega \in \Omega$ :

$$
\overline{\left\{f_{n}(\omega): n \in \mathbb{N}\right\}}=X_{\alpha}(\omega) \quad \text { (Castaing representation) }
$$

Proof: Most parts of the theorem can be proved following the proofs from Section 4.1. For a detailed proof compare [7].

Furthermore similar to the case of fuzzy random variables the measurability condition can also be expressed equivalently via the measurability of the $\alpha$-cut mappings $X_{\alpha}$ with respect to the Borel $\sigma$-algebra $\mathcal{B}\left(\left(\mathcal{K}_{c}^{d}, \delta_{H}\right)\right)$ in $\mathcal{K}_{c}^{d}$ generated by the Hausdorff metric. Since this is a supplement to Theorem 4.17 the enumeration of Theorem 4.17 is carried on:

Theorem 4.18 Suppose that $(\Omega, \mathcal{A}, \mathcal{P})$ is a complete probability space and let $\mathcal{B}\left(\left(\mathcal{K}_{c}^{d}, \delta_{H}\right)\right)$ denote the Borel $\sigma$-algebra in $\mathcal{K}_{c}^{d}$ generated by the Hausdorff metric $\delta_{H}$. Then the following conditions are equivalent for a fuzzy vectorvalued mapping $X^{\star}: \Omega \rightarrow \mathcal{F}_{c}^{d}$ :

$1^{d} . X^{\star}$ is a d-dimensional fuzzy random vector according to Definition 4.16 .

$9^{d}$. For every $\alpha \in(0,1]$ the $\alpha$-cut mapping $X_{\alpha}: \Omega \rightarrow \mathcal{K}_{c}^{d}$ is measurable with respect to $\mathcal{B}\left(\left(\mathcal{K}_{c}^{d}, \delta_{H}\right)\right)$.

Proof: The theorem can easily be proved following the proof of Theorem 4.13 and simply replacing $\mathcal{F}_{c}^{1}$ by $\mathcal{F}_{c}^{d}, \mathbb{R}$ by $\mathbb{R}^{d}, \mathcal{K}_{c}^{1}$ by $\mathcal{K}_{c}^{d}$ and $\mathscr{E}_{2, c}^{1}$ by $\mathscr{E}_{2, c}^{d}$.

Within the next theorem, which is an analogon to Theorem 4.14, as before $\operatorname{int}(A)$ denotes the topological interior of $A$, i.e. the set of all inner points of $A$, and $\operatorname{cls}(A)$ denoted the closure of $A$. i.e. the intersection of all closed sets containing $A$, for every set $A \subseteq \mathbb{R}^{d}$.

Theorem 4.19 Suppose that $(\Omega, \mathcal{A}, \mathcal{P})$ is a complete probability space and that $X^{\star}: \Omega \rightarrow \mathcal{F}_{c}^{d}$ is a fuzzy vector-valued mapping on $\Omega$ such that for $\mathcal{P}$. almost every $\omega \in \Omega \operatorname{int}\left(X_{1}(\omega)\right) \neq \emptyset$ holds. Then the following conditions are equivalent: 
$1^{d} . X^{\star}$ is a d-dimensional fuzzy random vector according to Definition 4.16.

10 $0^{d} .\left\{\omega \in \Omega: x \in X_{\alpha}(\omega)\right\} \in \mathcal{A}$ holds for every $\alpha \in(0,1]$ and every $x \in \mathbb{R}^{d}$.

Proof: Again one part of the equivalence is trivial:

If $X^{\star}$ is a fuzzy random vector then $\left\{\omega \in \Omega: x \in X_{\alpha}(\omega)\right\} \in \mathcal{A}$ surely holds since $\{x\}$ is closed and $x \in X_{\alpha}(\omega)$ is equivalent to $X_{\alpha}(\omega) \cap\{x\} \neq \emptyset$ for every $x \in \mathbb{R}^{d}$ and $\alpha \in(0,1]$.

On the other hand because of the fact that by assumption $\operatorname{int}\left(X_{1}(\omega)\right) \neq \emptyset$ holds for $\mathcal{P}$-almost every $\omega \in \Omega$ there exists a set $N \in \mathcal{A}$ with $\mathcal{P}(N)=0$ such that for all $\omega \in N^{c} \operatorname{int}\left(X_{\alpha}(\omega)\right) \neq \emptyset$ is fulfilled.

As it is well known that every convex compact set with non-empty interior coincides with the closure of its inner points (compare for instance [37]) it follows immediately that $X_{\alpha}(\omega)=\operatorname{cls}\left(\operatorname{int}\left(X_{\alpha}(\omega)\right)\right)$ holds for every $\omega \in N^{c}$ and every $\alpha \in(0,1]$.

Suppose now that $G$ is open, $\alpha \in(0,1]$ and that condition $10^{d}$ is fulfilled. Obviously the following equality holds:

$$
\begin{aligned}
\left\{\omega \in \Omega: X_{\alpha}(\omega) \cap G \neq \emptyset\right\} & =\overbrace{\left\{\omega \in N: X_{\alpha}(\omega) \cap G \neq \emptyset\right\}}^{=: A_{1}} \cup \\
& \cup \underbrace{\left\{\omega \in N^{c}: X_{\alpha}(\omega) \cap G \neq \emptyset\right\}}_{=: A_{2}}
\end{aligned}
$$

As a subset of the set $N$ of probability zero the set $A_{1}$ itself is measurable, i.e. $A_{1} \in \mathcal{A}$ holds. Consequently it suffices to prove that $A_{2}$ satisfies $A_{2} \in \mathcal{A}$, which can be done as follows:

$$
\begin{aligned}
A_{2}=\left\{\omega \in N^{c}: X_{\alpha}(\omega) \cap G \neq \emptyset\right\} & =\left\{\omega \in N^{c}: \operatorname{int}\left(X_{\alpha}(\omega)\right) \cap G \neq \emptyset\right\} \\
& =\left\{\omega \in N^{c}: \operatorname{int}\left(X_{\alpha}(\omega)\right) \cap G \cap \mathbb{Q}^{d} \neq \emptyset\right\} \\
& =N^{c} \cap \bigcup_{x \in \mathbb{Q}^{d} \cap G}\left\{\omega \in \Omega: x \in X_{\alpha}(\omega)\right\} \in \mathcal{A}
\end{aligned}
$$

This completes the proof.

Section 4.2 will be closed with the following theorem:

Theorem 4.20 Suppose that $(\Omega, \mathcal{A}, \mathcal{P})$ is a complete probability space and that $X^{\star}: \Omega \rightarrow \mathcal{F}_{c, c}^{d}$ is a fuzzy vector-valued mapping on $\Omega$. Furthermore denote by $\mathcal{B}\left(\left(\mathcal{F}_{c, c}^{d}, \delta_{H, \infty}^{\star}\right)\right)$ the $\sigma$-algebra generated by the metric $\delta_{H, \infty}^{\star}$ on $\mathcal{F}_{c, c}^{d}$. If $X^{\star}$ is measurable with respect to $\mathcal{B}\left(\left(\mathcal{F}_{c, c}^{d}, \delta_{H, \infty}^{\star}\right)\right)$ then $X^{\star}$ is a d-dimensional fuzzy random vector according to Definition 4.16. 
Proof: For every $\alpha \in(0,1]$ define a mapping $\Pi_{\alpha}: \mathcal{F}_{c, c}^{d} \rightarrow \mathcal{K}_{c}^{d}$ by setting $\Pi_{\alpha}\left(\xi^{\star}\right):=\left[\xi^{\star}\right]_{\alpha}$ for every $\xi^{\star} \in \mathcal{F}_{c, c}^{d}$. It follows immediately from the construction of $\delta_{H, \infty}^{*}$ that $\Pi_{\alpha}$ is Lipschitz continuous with Lipschitz constant 1 (in fact $\Pi_{\alpha}$ is a projection). Consequently $\Pi_{\alpha}$ is $\mathcal{B}\left(\left(\mathcal{F}_{c, c}^{d}, \delta_{H, \infty}^{\star}\right)\right)-\mathcal{B}\left(\left(\mathcal{K}_{c}^{d}, \delta_{H}\right)\right)$ measurable. It is well known that the composition of two measurable mappings is itself measurable. Consequently Theorem 4.18 and the fact that

$$
X_{\alpha}(\omega)=\Pi_{\alpha} \circ X^{\star}(\omega)
$$

holds for every $\omega \in \Omega$ complete the proof of the theorem.

\subsection{Fuzzy probability distributions induced by fuzzy random variables and fuzzy ran- dom vectors}

In this section it will be demonstrated how fuzzy random variables and fuzzy random vectors naturally induce a fuzzy probability distribution according to Definition 2.19. Since fuzzy random variables can be regarded as special cases of fuzzy random vectors, only fuzzy random vectors will be considered.

Every $d$-dimensional fuzzy random vector $X^{\star}: \Omega \rightarrow \mathcal{F}_{c}^{d}$ induces families $\left(\underline{\pi}_{\alpha}\right)_{\alpha \in(0,1]}$ and $\left(\bar{\pi}_{\alpha}\right)_{\alpha \in(0,1]}$ of real-valued set-functions on $\mathcal{B}\left(\mathbb{R}^{d}\right)$ in the following way: For every $\alpha \in(0,1]$ and every $B \in \mathcal{B}\left(\mathbb{R}^{d}\right)$ define

$$
\begin{aligned}
& \underline{\pi}_{\alpha}(B):=\mathcal{P}\left(\left\{\omega \in \Omega: X_{\alpha}(\omega) \subseteq B\right\}\right) \\
& \bar{\pi}_{\alpha}(B):=\mathcal{P}\left(\left\{\omega \in \Omega: X_{\alpha}(\omega) \cap B \neq \emptyset\right\}\right) .
\end{aligned}
$$

Obviously for every $B \in \mathcal{B}\left(\mathbb{R}^{d}\right)$ and $\alpha \in(0,1] \underline{\pi}_{\alpha}(B) \leq \bar{\pi}_{\alpha}(B)$ holds.

Using the fact that $\mathcal{P}$ is a probability measure, this shows that $\left[\underline{\pi}_{\alpha}(B), \bar{\pi}_{\alpha}(B)\right]$ is a non-empty, compact subinterval of $[0,1]$ for every $\alpha \in(0,1]$ and every $B \in \mathcal{B}\left(\mathbb{R}^{d}\right)$.

Suppose for the moment that $B \in \mathcal{B}\left(\mathbb{R}^{d}\right)$ is fixed and that $\alpha, \beta \in(0,1], \alpha \leq \beta$, then it follows that $X_{\alpha}(\omega) \supseteq X_{\beta}(\omega)$ for every $\omega \in \Omega$, and that

$$
\left\{\omega \in \Omega: X_{\alpha}(\omega) \subseteq B\right\} \subseteq\left\{\omega \in \Omega: X_{\beta}(\omega) \subseteq B\right\},
$$

which shows that $\underline{\pi}_{\alpha}(B) \leq \underline{\pi}_{\beta}(B)$. Moreover

$$
\left\{\omega \in \Omega: X_{\beta}(\omega) \cap B \neq \emptyset\right\} \subseteq\left\{\omega \in \Omega: X_{\alpha}(\omega) \cap B \neq \emptyset\right\},
$$

which gives that $\bar{\pi}_{\beta}(B) \leq \bar{\pi}_{\alpha}(B)$.

This proves that for fixed $B \in \mathcal{B}\left(\mathbb{R}^{d}\right),\left(\left[\underline{\pi}_{\alpha}(B), \bar{\pi}_{\alpha}(B)\right]\right)_{\alpha \in(0,1]}$ is a nested, 
monontonically decrasing family of non-empty compact intervals in $\alpha$.

Unfortunately, in contradiction to [30], in general $\left(\left[\underline{\pi}_{\alpha}(B), \bar{\pi}_{\alpha}(B)\right]\right)_{\alpha \in(0,1]}$ is not a family of $\alpha$-cuts of a fuzzy number, as the following counter-example shows:

Example 4.21 Suppose that $(\Omega, \mathcal{A}, \mathcal{P})$ is an arbitrary probability space and let $\eta^{\star} \in \mathcal{F}_{c}^{1}$ be the triangular fuzzy number with $\alpha$-cuts $\left[\eta^{\star}\right]_{\alpha}=[\alpha-1,1-\alpha]$ for every $\alpha \in(0,1]$ (depicted in Figure 4.1).

Define $X^{\star}: \Omega \rightarrow \mathcal{F}_{c}^{1}$ by simply setting $X^{\star}(\omega)=\eta^{\star}$ for every $\omega \in \Omega$. Obviously $X^{\star}$ is a fuzzy random variable (the measurability condition obviously is fulfilled).

Figure 4.1: $\eta^{\star}$ and $B$ in Example 4.21

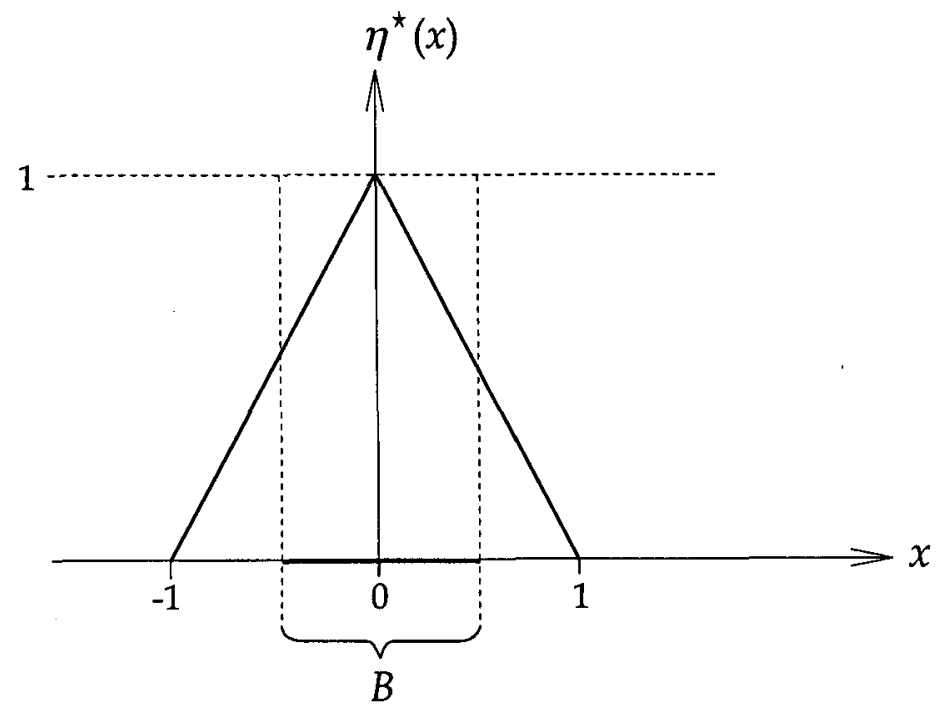

Choosing $B=\left[-\frac{1}{2}, \frac{1}{2}\right] \in \mathcal{B}(\mathbb{R})$ gives

$$
\left[\underline{\pi}_{\alpha}(B), \bar{\pi}_{\alpha}(B)\right]= \begin{cases}{[0,1]} & \text { if } \alpha<\frac{1}{2} \\ \{1\} & \text { if } \alpha \geq \frac{1}{2} .\end{cases}
$$

If the family $\left(\left[\underline{\pi}_{\alpha}(B), \bar{\pi}_{\alpha}(B)\right]\right)_{\alpha \in(0,1]}$ was a family of $\alpha$-cuts of a fuzzy number, then for every $\beta \in(0,1]$ the following would hold (compare Theorem 1.4)

$$
\left[\underline{\pi}_{\beta}(B), \bar{\pi}_{\beta}(B)\right]=\bigcap_{\alpha<\beta}\left[\underline{\pi}_{\alpha}(B), \bar{\pi}_{\alpha}(B)\right] .
$$


Choosing $\beta=\frac{1}{2}$ therefore would give

$$
\left[\underline{\pi}_{\frac{1}{2}}(B), \bar{\pi}_{\frac{1}{2}}(B)\right]=\bigcap_{\alpha<\frac{1}{2}}\left[\underline{\pi}_{\alpha}(B), \bar{\pi}_{\alpha}(B)\right]=[0,1],
$$

which is a contradiction to $\left[\underline{\pi}_{\frac{1}{2}}(B), \bar{\pi}_{\frac{1}{2}}(B)\right]=\{1\}$.

Consequently in general the family $\left(\left[\underline{\pi}_{\alpha}(B), \bar{\pi}_{\alpha}(B)\right]\right)_{\alpha \in(0,1]}$ need not be a family of $\alpha$-cuts of a fuzzy number, but because of the before mentioned properties one can easily construct a fuzzy number $\mathbb{P}^{\star}(B)$ for every $B \in \mathcal{B}\left(\mathbb{R}^{d}\right)$ by simply building the convex hull, i.e. for $x \in \mathbb{R}$ define

$$
\left(\mathbb{P}^{\star}(B)\right)(x):=\left\{\begin{array}{l}
0 \quad \text { if } x \notin\left[\underline{\pi}_{\alpha}(B), \bar{\pi}_{\alpha}(B)\right] \quad \forall \alpha \in(0,1] \\
\sup \left\{\alpha \in(0,1]: x \in\left(\left[\underline{\pi}_{\alpha}(B), \bar{\pi}_{\alpha}(B)\right]\right\}\right.
\end{array}\right. \text { otherwise. }
$$

Note that according to Theorem 1.36 the $\alpha$-cuts $\left[\mathbb{P}^{\star}(B)\right]_{\alpha}$ of $\mathbb{P}^{\star}(B)$ coincide with $\left[\underline{\pi}_{\alpha}(B), \bar{\pi}_{\alpha}(B)\right]$ for $\lambda$-almost every $\alpha \in(0,1]$.

This again defines a mapping $\mathbb{P}^{\star}: \mathcal{B}\left(\mathbb{R}^{d}\right) \rightarrow \mathcal{F}_{c}^{1}$ that satisfies Definition 2.19 on $\left(\mathbb{R}^{d}, \mathcal{B}\left(\mathbb{R}^{d}\right)\right)$ which is now going to be proved in three steps:

Firstly properties of the families $\left(\underline{\pi}_{\alpha}\right)_{\alpha \in(0,1]},\left(\bar{\pi}_{\alpha}\right)_{\alpha \in(0,1]}$ will be analyzed.

After that, a simple formula describing the interrelation between the $\alpha$-cuts of the fuzzy numbers $\mathbb{P}^{\star}(B)$ and the generating families $\left(\underline{\pi}_{\alpha}\right)_{\alpha \in(0,1]},\left(\bar{\pi}_{\alpha}\right)_{\alpha \in(0,1]}$ will be proved.

Finally it will be shown that $\mathbb{P}^{\star}$ satisfies Definition 2.19 on $\left(\mathbb{R}^{d}, \mathcal{B}\left(\mathbb{R}^{d}\right)\right)$ by using this formula and the properties of $\left(\underline{\pi}_{\alpha}\right)_{\alpha \in(0,1]},\left(\bar{\pi}_{\alpha}\right)_{\alpha \in(0,1]}$.

Lemma 4.22 Suppose that $(\Omega, \mathcal{A}, \mathcal{P})$ is an arbitrary probability space and that $X^{\star}: \Omega \rightarrow \mathcal{F}_{c}^{d}$ is a d-dimensional fuzzy random vector.

Then the families $\left(\underline{\pi}_{\alpha}\right)_{\alpha \in(0,1]}$ and $\left(\bar{\pi}_{\alpha}\right)_{\alpha \in(0,1]}$, defined according to (4.10), fulfill all points of Definition 2.20 on $\left(\mathbb{R}^{d}, \mathcal{B}\left(\mathbb{R}^{d}\right)\right)$.

Proof: Point one is surely satisfied since it follows immediately from the definition that

$$
\underline{\pi}_{\alpha}\left(\mathbb{R}^{d}\right)=\bar{\pi}_{\alpha}\left(\mathbb{R}^{d}\right)=1 \text { and } \underline{\pi}_{\alpha}(\emptyset)=\bar{\pi}_{\alpha}(\emptyset)=0 \quad \forall \alpha \in(0,1] .
$$

If $A, B \in \mathcal{B}\left(\mathbb{R}^{d}\right)$ satisfy $A \subseteq B$, then it follows that for every $\alpha \in(0,1]$

$$
\begin{aligned}
& \left\{\omega \in \Omega: X_{\alpha}(\omega) \subseteq A\right\} \subseteq\left\{\omega \in \Omega: X_{\alpha}(\omega) \subseteq B\right\} \quad \text { and } \\
& \left\{\omega \in \Omega: X_{\alpha}(\omega) \cap A \neq \emptyset\right\} \subseteq\left\{\omega \in \Omega: X_{\alpha}(\omega) \cap B \neq \emptyset\right\}
\end{aligned}
$$

which shows that $\underline{\pi}_{\alpha}(A) \leq \underline{\pi}_{\alpha}(B)$ and $\bar{\pi}_{\alpha}(A) \leq \bar{\pi}_{\alpha}(B)$ for every $\alpha \in(0,1]$. In order to prove the third assertion suppose that $A, B \in \mathcal{B}\left(\mathbb{R}^{d}\right)$ and that $A \cap B=\emptyset$. Then for every $\alpha \in(0,1]$ on the one hand it follows that

$$
\left(\left\{\omega \in \Omega: X_{\alpha}(\omega) \subseteq A\right\} \cup\left\{\omega \in \Omega: X_{\alpha}(\omega) \subseteq B\right\}\right) \subseteq\left\{\omega \in \Omega: X_{\alpha}(\omega) \subseteq A \cup B\right\},
$$


which shows that $\underline{\pi}_{\alpha}(A)+\underline{\pi}_{\alpha}(B) \leq \underline{\pi}_{\alpha}(A \cup B)$, and on the other hand

$$
\begin{aligned}
\left\{\omega \in \Omega: X_{\alpha}(\omega) \cap(A \cup B) \neq \emptyset\right\}= & \left\{\omega \in \Omega: X_{\alpha}(\omega) \cap A \neq \emptyset\right\} \cup \\
& \cup\left\{\omega \in \Omega: X_{\alpha}(\omega) \cap B \neq \emptyset\right\},
\end{aligned}
$$

which finally shows that $\bar{\pi}_{\alpha}(A \cup B) \leq \bar{\pi}_{\alpha}(A)+\bar{\pi}_{\alpha}(B)$.

The last assertion is an immediate consequence of the following identities:

$$
\begin{aligned}
\underline{\pi}_{\alpha}\left(A^{c}\right) & =\mathcal{P}\left(\left\{\omega \in \Omega: X_{\alpha}(\omega) \subseteq A^{c}\right\}=1-\mathcal{P}\left(\left\{\omega \in \Omega: X_{\alpha}(\omega) \nsubseteq A^{c}\right\}\right)\right. \\
& =1-\mathcal{P}\left(\left\{\omega \in \Omega: X_{\alpha}(\omega) \cap A \neq \emptyset\right\}\right)=1-\bar{\pi}_{\alpha}(A) \\
\bar{\pi}_{\alpha}\left(A^{c}\right)= & \mathcal{P}\left(\left\{\omega \in \Omega: X_{\alpha}(\omega) \cap A \neq \emptyset\right\}=1-\mathcal{P}\left(\left\{\omega \in \Omega: X_{\alpha}(\omega) \cap A^{c}=\emptyset\right\}\right)\right. \\
= & 1-\mathcal{P}\left(\left\{\omega \in \Omega: X_{\alpha}(\omega) \subseteq A\right\}\right)=1-\underline{\pi}_{\alpha}(A)
\end{aligned}
$$

As already mentioned before for every $d$-dimensional Borel set $B \in \mathcal{B}\left(\mathbb{R}^{d}\right)$ the family $\left(\left[\underline{\pi}_{\alpha}(B), \bar{\pi}_{\alpha}(B)\right]\right)_{\alpha \in(0,1]}$ induces a fuzzy number $\mathbb{P}^{\star}(B) \in \mathcal{F}_{c}^{1}$ in the following way: for $x \in \mathbb{R}$ define

$$
\left(\mathbb{P}^{\star}(B)\right)(x):=\left\{\begin{array}{l}
0 \quad \text { if } x \notin\left[\underline{\pi}_{\alpha}(B), \bar{\pi}_{\alpha}(B)\right] \quad \forall \alpha \in(0,1] \\
\sup \left\{\alpha \in(0,1]: x \in\left(\left[\underline{\pi}_{\alpha}(B), \bar{\pi}_{\alpha}(B)\right]\right\}\right.
\end{array}\right. \text { otherwise. }
$$

The $\alpha$-cuts of the resulting fuzzy number will be denoted by $\left[\underline{p}_{\alpha}(B), \bar{p}_{\alpha}(B)\right]$, i.e.

$$
\left[\underline{p}_{\alpha}(B), \bar{p}_{\alpha}(B)\right]:=\left[\mathbb{P}^{\star}(B)\right]_{\alpha}, \quad \text { for } B \in \mathcal{B}\left(\mathbb{R}^{d}\right) \text { and } \alpha \in(0,1] \text {. }
$$

For every $B \in \mathcal{B}\left(\mathbb{R}^{d}\right)$ there is a simple interrelation between the family $\left(\left[\underline{p}_{\alpha}(B), \bar{p}_{\alpha}(B)\right]\right)_{\alpha \in(0,1]}$ and the generating family $\left(\left[\underline{\pi}_{\alpha}(B), \bar{\pi}_{\alpha}(B)\right]\right)_{\alpha \in(0,1]}$.

In fact the following lemma holds:

Lemma 4.23 Let $\alpha$ be an arbitrary but fixed real number in $(0,1]$ and suppose that $\left(\alpha_{n}\right)_{n \in \mathbb{N}}$ is a strictly increasing sequence in $(0,1)$ that converges to $\alpha$. Then the following equation holds for every $B \in \mathcal{B}\left(\mathbb{R}^{d}\right)$ :

$$
\begin{aligned}
\left.\underline{p}_{\alpha}(B), \bar{p}_{\alpha}(B)\right] & =\bigcap_{\beta<\alpha}\left[\underline{\pi}_{\alpha}(B), \bar{\pi}_{\alpha}(B)\right]=\bigcap_{n=1}^{\infty}\left[\underline{\pi}_{\alpha_{n}}(B), \bar{\pi}_{\alpha_{n}}(B)\right] \\
& =\left[\lim _{n \rightarrow \infty} \underline{\pi}_{\alpha_{n}}(B), \lim _{n \rightarrow \infty} \bar{\pi}_{\alpha_{n}}(B)\right]
\end{aligned}
$$

Proof: The first equality has already been proved for more general cases in Lemma 1.4 .

In order to prove the second equality suppose that $\beta<\alpha$. Then by definition 
of the sequence $\left(\alpha_{n}\right)_{n \in \mathbb{N}}$ there surely exists $n_{0}(\beta) \in \mathbb{N}$ such that for all $n \geq n_{0}$ $\alpha_{n} \in(\beta, \alpha)$ holds. This proves that $\left[\underline{\pi}_{\alpha_{n}}(B), \bar{\pi}_{\alpha_{n}}(B)\right] \subseteq\left[\underline{\pi}_{\beta}(B), \bar{\pi}_{\beta}(B)\right]$ for all $n \geq n_{0}$. Since $\beta<\alpha$ was arbitrary it follows that

$$
\bigcap_{\beta<\alpha}\left[\underline{\pi}_{\alpha}(B), \bar{\pi}_{\alpha}(B)\right] \subseteq \bigcap_{n=1}^{\infty}\left[\underline{\pi}_{\alpha_{n}}(B), \bar{\pi}_{\alpha_{n}}(B)\right] .
$$

Since the reverse inclusion is obvious because $\alpha_{n} \in(0, \alpha)$ holds for every $n \in \mathbb{N}$ by assumption, this proves the second equality.

Concerning the third equality first of all notice that the mentioned limits really exist, since for every $B \in \mathcal{B}\left(\mathbb{R}^{d}\right)$ the sequence $\underline{\pi}_{\alpha_{n}}(B)$ is monotonically increasing and bounded and the sequence $\bar{\pi}_{\alpha_{n}}(B)$ is monotonically decreasing and bounded.

$x \in \bigcap_{n=1}^{\infty}\left[\underline{\pi}_{\alpha_{n}}(B), \bar{\pi}_{\alpha_{n}}(B)\right]$ means that $\underline{\pi}_{\alpha_{n}}(B) \leq x \leq \bar{\pi}_{\alpha_{n}}(B)$ for all $n \in \mathbb{N}$, from which it follows immediately that $\lim _{n \rightarrow \infty} \underline{\pi}_{\alpha_{n}}(B) \leq x \leq \lim _{n \rightarrow \infty} \bar{\pi}_{\alpha_{n}}(B)$, which proves that

$$
\bigcap_{n=1}^{\infty}\left[\underline{\pi}_{\alpha_{n}}(B), \bar{\pi}_{\alpha_{n}}(B)\right] \subseteq\left[\lim _{n \rightarrow \infty} \underline{\pi}_{\alpha_{n}}(B), \lim _{n \rightarrow \infty} \bar{\pi}_{\alpha_{n}}(B)\right] .
$$

Because of the fact that the other inclusion is an immediate consequence of the monotony on the sequences $\underline{\pi}_{\alpha_{n}}(B)$ and $\bar{\pi}_{\alpha_{n}}(B)$ this concludes the proof of the lemma.

Since for every $\alpha \in(0,1]$ there surely exists a sequence $\left(\alpha_{n}\right)_{n \in \mathbb{N}}$ in $(0,1)$ that is strictly monotonically increasing and converging to $\alpha$ like stated in Lemma 4.23 is follows that for every $B \in \mathcal{B}\left(\mathbb{R}^{d}\right)$ the following equations hold:

$$
\begin{aligned}
& \underline{p}_{\alpha}(B)=\lim _{n \rightarrow \infty} \underline{\pi}_{\alpha_{n}}(B) \quad \text { and } \\
& \bar{p}_{\alpha}(B)=\lim _{n \rightarrow \infty} \bar{\pi}_{\alpha_{n}}(B)
\end{aligned}
$$

This fact can be used to prove the following result:

Theorem 4.24 Suppose that $(\Omega, \mathcal{A}, \mathcal{P})$ is an arbitrary probability space and that $X^{\star}: \Omega \rightarrow \mathcal{F}_{c}^{d}$ is a d-dimensional fuzzy random vector. For every $B \in \mathcal{B}\left(\mathbb{R}^{d}\right)$ and $\alpha \in(0,1]$ let $\underline{\pi}_{\alpha}(B)$ and $\bar{\pi}_{\alpha}(B)$ be defined according to (4.10), $\mathbb{P}^{\star}(B)$ defined according to (4.11), and $\underline{p}_{\alpha}(B)$ and $\bar{p}_{\alpha}(B)$ defined according to (4.12). Then $\mathbb{P}^{\star}: \mathcal{B}\left(\mathbb{R}^{d}\right) \rightarrow \mathcal{F}_{c}^{1}$ is a fuzzy probability distribution in the sense of Definition 2.19.

(Or equivalently $\underline{p}_{\alpha}$ and $\bar{p}_{\alpha}$ fulfill Definition 2.20.) 
Proof: Let $\alpha \in(0,1]$ be arbitrary. Choose a sequence $\left(\alpha_{n}\right)_{n \in \mathbb{N}}$ in $(0,1)$ that is strictly monotonically increasing and converges to $\alpha$. Using the fact that

$$
\underline{\pi}_{\alpha}\left(\mathbb{R}^{d}\right)=\bar{\pi}_{\alpha}\left(\mathbb{R}^{d}\right)=1 \text { and } \underline{\pi}_{\alpha}(\emptyset)=\bar{\pi}_{\alpha}(\emptyset)=0 \quad \forall \alpha \in(0,1],
$$

applying (4.13) immediately yields $\underline{p}_{\alpha}\left(\mathbb{R}^{d}\right)=\bar{p}_{\alpha}\left(\mathbb{R}^{d}\right)=1$ and $\underline{p}_{\alpha}(\emptyset)=\bar{p}_{\alpha}(\emptyset)=0$. Furthermore if $A, B \in \mathcal{B}\left(\mathbb{R}^{d}\right)$ with $A \subseteq B$, then again applying (4.13) immediately shows that

$$
\begin{aligned}
& \underline{p}_{\alpha}(A)=\lim _{n \rightarrow \infty} \underline{\pi}_{\alpha_{n}}(A) \leq \lim _{n \rightarrow \infty} \underline{\pi}_{\alpha_{n}}(B)=\underline{p}_{\alpha}(B) \quad \text { and } \\
& \bar{p}_{\alpha}(A)=\lim _{n \rightarrow \infty} \bar{\pi}_{\alpha_{n}}(A) \leq \lim _{n \rightarrow \infty} \bar{\pi}_{\alpha_{n}}(B)=\bar{p}_{\alpha}(B),
\end{aligned}
$$

which proves point two of Definition 2.20.

Finally if $A \cap B=\emptyset$ for two Borel sets $A, B \in \mathcal{B}\left(\mathbb{R}^{d}\right)$, then it follows again by using (4.13) and Lemma 4.23 that

$$
\begin{aligned}
\underline{p}_{\alpha}(A \cup B) & =\lim _{n \rightarrow \infty} \underline{\pi}_{\alpha_{n}}(A \cup B) \geq \lim _{n \rightarrow \infty}\left(\underline{\pi}_{\alpha_{n}}(A)+\underline{\pi}_{\alpha_{n}}(B)\right) \\
& =\underline{p}_{\alpha}(A)+\underline{p}_{\alpha}(B) \quad \text { and } \\
\bar{p}_{\alpha}(A \cup B) & =\lim _{n \rightarrow \infty} \bar{\pi}_{\alpha_{n}}(A \cup B) \leq \lim _{n \rightarrow \infty}\left(\bar{\pi}_{\alpha_{n}}(A)+\bar{\pi}_{\alpha_{n}}(B)\right) \\
& =\bar{p}_{\alpha}(A)+\bar{p}_{\alpha}(B) .
\end{aligned}
$$

Finally again using (4.13) and Lemma 4.23 shows that

$$
\begin{aligned}
\underline{p}_{\alpha}\left(A^{c}\right) & =\lim _{n \rightarrow \infty} \underline{\pi}_{\alpha_{n}}\left(A^{c}\right)=\lim _{n \rightarrow \infty}\left(1-\bar{\pi}_{\alpha_{n}}(A)\right) \\
& =1-\bar{p}_{\alpha}(A) \quad \text { and } \\
\bar{p}_{\alpha}\left(A^{c}\right) & =\lim _{n \rightarrow \infty} \bar{\pi}_{\alpha_{n}}\left(A^{c}\right)=\lim _{n \rightarrow \infty}\left(1-\underline{\pi}_{\alpha_{n}}(A)\right) \\
& =1-\underline{p}_{\alpha}(A) .
\end{aligned}
$$

This completes the proof.

Remark: It will be proved in Section 5.3 that for every Borel set $B \in \mathcal{B}\left(\mathbb{R}^{d}\right)$ with probability one $\mathbb{P}^{\star}(B)$, defined according to (4.11), is the limit of relative frequencies $h_{n}^{\star}(B, \omega)$ (defined as in Section 2.1) induced by a sequence $\left(X_{n}^{\star}\right)_{n \in \mathbb{N}}$ of pairwise independent, identically distributed $d$-dimensional fuzzy random vectors. In other words, a Strong Law of Large Numbers holds.

Theorem 4.24 can be seen as a further justification of the general definition of a fuzzy probability distribution (Definition 2.19) since the fuzzy-valued set-functions (probabilities) arising from two completely different approaches (using densities respectively fuzzy random vectors) both are included in the 
general definition.

Regarding Theorem 2.18 the question arises whether $\underline{p}_{\alpha}$ is even super- $\sigma$ additive and whether $\bar{p}_{\alpha}$ is even sub- $\sigma$-additive for fixed $\alpha \in(0,1]$ (both defined according to (4.12)).

As a first step going in that direction, the following theorem can be proved:

Lemma 4.25 Suppose that $(\Omega, \mathcal{A}, \mathcal{P})$ is an arbitrary probability space and that $X^{\star}: \Omega \rightarrow \mathcal{F}_{c}^{d}$ is a d-dimensional fuzzy random vector. Furthermore for every $\alpha \in(0,1]$ let $\underline{\pi}_{\alpha}$ and $\bar{\pi}_{\alpha}$ be defined according to (4.10).

Then for every $\alpha \in(0,1] \underline{\pi}_{\alpha}$ is super- $\sigma$-additive and $\bar{\pi}_{\alpha}$ is sub- $\sigma$-additive, i.e. if $\left(B_{n}\right)_{n \in \mathbb{N}}$ is a pairwise disjoint sequence of Borel subsets of $\mathbb{R}^{d}$, then for every $\alpha \in(0,1]$ the following holds:

$$
\underline{\pi}_{\alpha}\left(\bigcup_{n=1}^{\infty} B_{n}\right) \geq \sum_{n=1}^{\infty} \underline{\pi}_{\alpha}\left(B_{n}\right), \quad \bar{\pi}_{\alpha}\left(\bigcup_{n=1}^{\infty} B_{n}\right) \leq \sum_{n=1}^{\infty} \bar{\pi}_{\alpha}\left(B_{n}\right)
$$

Proof: Suppose that $\left(B_{n}\right)_{n \in \mathbb{N}}$ is a sequence of pairwise disjoint Borel subsets of $\mathbb{R}^{d}$ and let $\alpha \in(0,1]$ be arbitrary, then super- $\sigma$-additivity of $\pi_{\alpha}$ can easily be shown by using the fact that $\mathcal{P}$ is a probability measure on $(\Omega, \mathcal{A})$ in the following way:

$$
\begin{aligned}
\underline{\pi}_{\alpha}\left(\bigcup_{n=1}^{\infty} B_{n}\right) & =\mathcal{P}\left(\left\{\omega \in \Omega: X_{\alpha}(\omega) \subseteq \bigcup_{n=1}^{\infty} B_{n}\right\}\right) \\
& \geq \mathcal{P}\left(\bigcup_{n=1}^{\infty}\left\{\omega \in \Omega: X_{\alpha}(\omega) \subseteq B_{n}\right\}\right) \\
& =\sum_{n=1}^{\infty} \mathcal{P}\left(\left\{\omega \in \Omega: X_{\alpha}(\omega) \subseteq B_{n}\right\}\right) \\
& =\sum_{n=1}^{\infty} \underline{\pi}_{\alpha}\left(B_{n}\right)
\end{aligned}
$$

In the same manner one can prove sub- $\sigma$-additivity of $\bar{\pi}_{\alpha}$ :

$$
\begin{aligned}
\bar{\pi}_{\alpha}\left(\bigcup_{n=1}^{\infty} B_{n}\right) & =\mathcal{P}\left(\left\{\omega \in \Omega: X_{\alpha}(\omega) \cap \bigcup_{n=1}^{\infty} B_{n} \neq \emptyset\right\}\right) \\
& =\mathcal{P}\left(\bigcup_{n=1}^{\infty}\left\{\omega \in \Omega: X_{\alpha}(\omega) \cap B_{n} \neq \emptyset\right\}\right) \\
& \leq \sum_{n=1}^{\infty} \mathcal{P}\left(\left\{\omega \in \Omega: X_{\alpha}(\omega) \cap B_{n} \neq \emptyset\right\}\right) \\
& =\sum_{n=1}^{\infty} \bar{\pi}_{\alpha}\left(B_{n}\right)
\end{aligned}
$$


Having this, it can be proved that $\underline{p}$ defined according to (4.11) and (4.12) is even super- $\sigma$-additive for every $\alpha \in(0,1]$ :

Theorem 4.26 Suppose that $(\Omega, \mathcal{A}, \mathcal{P})$ is an arbitrary probability space and that $X^{\star}: \Omega \rightarrow \mathcal{F}_{c}^{d}$ is a d-dimensional fuzzy random vector. For every $B \in \mathcal{B}\left(\mathbb{R}^{d}\right)$ and $\alpha \in(0,1]$ let $\underline{\pi}_{\alpha}(B)$ be defined according to $(4.10), \mathbb{P}^{\star}(B)$ defined according to (4.11) and $\underline{p}_{\alpha}(B)$ defined according to (4.12).

Then $\underline{p}_{\alpha}$ is super- $\sigma$-additive for every $\alpha \in(0,1]$.

Proof: Suppose again that $\left(B_{i}\right)_{i \in \mathbb{N}}$ is a sequence of pairwise disjoint Borel sets, let $\alpha \in(0,1]$ be arbitrary and choose $\left(\alpha_{n}\right)_{n \in \mathbb{N}}$ according to Lemma 4.23. For every $n \in \mathbb{N}$ define a function $f_{n}: \mathbb{N} \rightarrow \mathbb{R}$ by $f_{n}(i)=\underline{\pi}_{\alpha_{n}}\left(B_{i}\right)$ and a function $f: \mathbb{N} \rightarrow \mathbb{R}$ by $f(i)=\underline{p}_{\alpha}\left(B_{i}\right)$, for every $i \in \mathbb{N}$.

Furthermore consider the measure space $\left(\mathbb{N}, 2^{\mathbb{N}}, \tau\right)$, where $2^{\mathbb{N}}$ is the set of all subsets of $\mathbb{N}$ and $\tau$ denotes the counting measure on $2^{\mathbb{N}}$.

Obviously all functions $f_{n}, n \in \mathbb{N}$, and $f$ are measurable, $f_{n}$ is monotonically increasing and $\lim _{n \rightarrow \infty} f_{n}(i)=f(i)$ for every $i \in \mathbb{N}$ by construction.

Applying the monotone convergence theorem (compare for instance [13]) it follows that

$$
\lim _{n \rightarrow \infty} \int_{\mathbb{N}} f_{n}(t) d \tau(t)=\int_{\mathbb{N}} f(t) d \tau(t)
$$

Since by construcion

$$
\int_{\mathbb{N}} f_{n}(t) d \tau(t)=\sum_{i=1}^{\infty} \underline{\pi}_{\alpha_{n}}\left(B_{i}\right) \quad \text { and } \quad \int_{\mathbb{N}} f(t) d \tau(t)=\sum_{i=1}^{\infty} \underline{p}_{\alpha}\left(B_{i}\right)
$$

this proves that

$$
\lim _{n \rightarrow \infty} \sum_{i=1}^{\infty} \underline{\pi}_{\alpha_{n}}\left(B_{i}\right)=\sum_{i=1}^{\infty} \underline{p}_{\alpha}\left(B_{i}\right)
$$

Consequently

$$
\underline{p}_{\alpha}\left(\bigcup_{i=1}^{\infty} B_{i}\right)=\lim _{n \rightarrow \infty} \underline{\pi}_{\alpha_{n}}\left(\bigcup_{i=1}^{\infty} B_{i}\right) \geq \lim _{n \rightarrow \infty} \sum_{i=1}^{\infty} \underline{\pi}_{\alpha_{n}}\left(B_{i}\right)=\sum_{i=1}^{\infty} \underline{p}_{\alpha}\left(B_{i}\right)
$$

which completes the proof of the theorem.

The remaining part of this section deals with regularity and continuity properties of the real-valued set functions $\underline{\pi}_{\alpha}(\cdot)$ and $\bar{\pi}_{\alpha}(\cdot)$ defined according to (4.10) induced by a fuzzy random vector $X^{\star}: \Omega \rightarrow \mathcal{F}_{c}^{d}$ (compare [29]).

Theorem 4.27 Suppose that $(\Omega, \mathcal{A}, \mathcal{P})$ is a complete probability space and that $X^{\star}: \Omega \rightarrow \mathcal{F}_{c}^{d}$ is a d-dimensional fuzzy random vector. For every $B \in \mathcal{B}\left(\mathbb{R}^{d}\right)$ and $\alpha \in(0,1]$ let $\bar{\pi}_{\alpha}(B)$ be defined according to (4.10) then: 
- For every open set $G \subseteq \mathbb{R}^{d}$ and every $\alpha \in(0,1]$ the following equality holds:

$$
\bar{\pi}_{\alpha}(G)=\sup \left\{\bar{\pi}_{\alpha}(K): K \subseteq G, K \in \mathcal{K}^{d}\right\}
$$

- For every Borel set $B \in \mathcal{B}\left(\mathbb{R}^{d}\right)$ and every $\alpha \in(0,1]$ the following equality holds:

$$
\bar{\pi}_{\alpha}(B)=\sup \left\{\bar{\pi}_{\alpha}(K): K \subseteq B, K \in \mathcal{K}^{d}\right\}=\inf \left\{\bar{\pi}_{\alpha}(G): G \supseteq B, G \text { open }\right\}
$$

Proof: It is obvious that the second assertion implies the first assertion. Nevertheless the assertions are stated separatedly since the first assertion will be proved completely, whereas the proof of the second assertion uses a very non-trivial result from the theory of capacities, which will not be proved here.

In order to prove the first assertion it suffices to show that

$$
\bar{\pi}_{\alpha}(G) \leq \sup \left\{\bar{\pi}_{\alpha}(K): K \subseteq G, K \in \mathcal{K}^{d}\right\}
$$

since the other inequality is an immediate consequence of Lemma 4.22.

As in Lemma 1.18 suppose that $\mathfrak{B}$ denotes the countable basis for the Euclidean topology on $\mathbb{R}^{d}$ consisting of open all balls $B(m, r)$ with $m \in \mathbb{Q}^{d}$ and $r \in \mathbb{Q}^{+}$. Then it follows immediately that every open set $G \subseteq \mathbb{R}^{d}$ can be represented as follows ( $\bar{B}$ denotes the corresponding closed ball):

$$
G=\bigcup_{B \in \mathfrak{B}, \bar{B} \subseteq G} \bar{B}
$$

Let the corresponding balls $B \in \mathfrak{B}$ fulfilling $\bar{B} \subseteq G$ be enumerated as $\left\{B_{1}, B_{2}, \cdots\right\}$. Using the notation explained in (4.4), equation (4.5), and the fact that every probability measure is continuous (compare [2]), then it follows immediately that

$$
\begin{aligned}
\bar{\pi}_{\alpha}(G) & =\mathcal{P}\left(\left\{\omega \in \Omega: X_{\alpha}(\omega) \cap G \neq \emptyset\right\}\right)=\mathcal{P}\left(X_{\alpha}^{\sim 1}(G)\right) \\
& =\mathcal{P}\left(X_{\alpha}^{\sim 1}\left(\bigcup_{i=1}^{\infty} \bar{B}_{i}\right)\right)=\lim _{n \rightarrow \infty} \mathcal{P}\left(X_{\alpha}^{\sim 1}\left(\bigcup_{i=1}^{n} \bar{B}_{i}\right)\right) \\
& =\lim _{n \rightarrow \infty} \bar{\pi}_{\alpha}\left(\bigcup_{i=1}^{n} \bar{B}_{i}\right) .
\end{aligned}
$$

Since for every $B \in \mathfrak{B}$ the closure $\bar{B}$ is compact (it is closed and bounded) this implies that $\bigcup_{i=1}^{n} \bar{B}_{i}$ is compact for every $n \in \mathbb{N}$, which completes the proof of inequality (4.14), and therefore the proof of the first assertion of 
Theorem 4.27.

The second (much stronger) assertion can be proved using the so-called Choquet Capacitability Theorem (compare [8] and [29]), which implies that

$$
\sup \left\{\bar{\pi}_{\alpha}(K): K \subseteq B, K \in \mathcal{K}^{d}\right\}=\inf \left\{\tilde{\pi}_{\alpha}(G): G \supseteq B, G \text { open in } \mathbb{R}^{d}\right\}
$$

holds for every Borel set $B \in \mathcal{B}\left(\mathbb{R}^{d}\right)$. Again using monotonicity of $\bar{\pi}_{\alpha}(\cdot)$ (Lemma 4.22) the desired equality immediately follows.

Concerning continuity properties of $\bar{\pi}_{\alpha}(\cdot)$ the following result holds:

Theorem 4.28 Suppose that $(\Omega, \mathcal{A}, \mathcal{P})$ is a complete probability space and that $X^{\star}: \Omega \rightarrow \mathcal{F}_{c}^{d}$ is a d-dimensional fuzzy random vector. For every $B \in \mathcal{B}\left(\mathbb{R}^{d}\right)$ and $\alpha \in(0,1]$ let $\bar{\pi}_{\alpha}(B)$ be defined according to (4.10) then:

- The set function $\bar{\pi}_{\alpha}(\cdot)$ is continuous from below, i.e. if $\left(B_{n}\right)_{n \in \mathbb{N}}$ is a monotonically increasing sequence of Borel sets with limit $B$, then $\lim _{n \rightarrow \infty} \bar{\pi}_{\alpha}\left(B_{n}\right)=\bar{\pi}_{\alpha}(B)$ holds.

- The set function $\bar{\pi}_{\alpha}(\cdot)$ is continuous from above for closed sets, i.e. if $\left(F_{n}\right)_{n \in \mathbb{N}}$ is a monotonically decreasing sequence of closed sets with limit $F$, then $\lim _{n \rightarrow \infty} \bar{\pi}_{\alpha}\left(F_{n}\right)=\bar{\pi}_{\alpha}(F)$ holds.

- In general $\pi_{\alpha}(\cdot)$ is not continuous from above.

Proof: Suppose that $\left(B_{n}\right)_{n \in \mathbb{N}}$ is an increasing sequence of Borel sets in $\mathbb{R}^{d}$ with limit $B=\bigcup_{n=1}^{\infty} B_{n} \in \mathcal{B}\left(\mathbb{R}^{d}\right)$, then again using the notation explained in (4.4) and equation (4.5) continuity from below follows immediately by

$$
\begin{aligned}
\bar{\pi}_{\alpha}(B) & =\mathcal{P}\left(\left\{\omega \in \Omega: X_{\alpha}(\omega) \cap B \neq \emptyset\right\}\right)=\mathcal{P}\left(X_{\alpha}^{\sim 1}(B)\right) \\
& =\mathcal{P}\left(X_{\alpha}^{\sim 1}\left(\bigcup_{i=1}^{\infty} B_{i}\right)\right)=\lim _{n \rightarrow \infty} \mathcal{P}\left(X_{\alpha}^{\sim 1}\left(B_{n}\right)\right) \\
& =\lim _{n \rightarrow \infty} \bar{\pi}_{\alpha}\left(B_{n}\right) .
\end{aligned}
$$

In order to prove the second assertion assume that $\left(F_{n}\right)_{n \in \mathbb{N}}$ is a monotonically decreasing sequence of closed sets with limit $F=\bigcap_{n=1}^{\infty} F_{n}$. Note that since $X_{\alpha}(\omega) \in \mathcal{K}_{c}^{d}$ holds for every $\omega \in \Omega$ and every $\alpha \in(0,1]$ by defnition and since $F_{n}$ is closed for every $n \in \mathbb{N}$ the set $X_{\alpha}(\omega) \cap F_{n}$ is compact. Consequently $X_{\alpha}(\omega) \cap F_{n} \neq \emptyset$ for every $n \in \mathbb{N}$ implies that $X_{\alpha}(\omega) \cap F \neq \emptyset$, which yields that $X_{\alpha}^{\sim 1}\left(F_{n}\right)$ monotonically decreases to $X_{\alpha}^{\sim 1}(F)$. Having that the second assertion of Theorem 4.28 follows immediately from

$$
\bar{\pi}_{\alpha}(F)=\mathcal{P}\left(X_{\alpha}^{\sim 1}(F)\right)=\lim _{n \rightarrow \infty} \mathcal{P}\left(X_{\alpha}^{\sim 1}\left(F_{n}\right)\right)=\lim _{n \rightarrow \infty} \bar{\pi}_{\alpha}\left(F_{n}\right) .
$$


The fact that in general $\bar{\pi}_{\alpha}(\cdot)$ is not continuous from above can easily be demonstrated as follows:

Suppose that $(\Omega, \mathcal{A}, \mathcal{P})$ is a complete probability space, define a fuzzy random variable $X^{\star}: \Omega \rightarrow \mathcal{F}_{c}^{1}$ by setting $X^{\star}(\omega):=\mathbf{1}_{[1,2]}$ for every $\omega \in \Omega$, and define for every $n \in \mathbb{N}$ an open set $G_{n}$ by $G_{n}:=\left(1,1+\frac{1}{n}\right)$.

Then obviously $\bigcap_{n=1}^{\infty} G_{n}=\emptyset$ and $\bar{\pi}_{\alpha}\left(G_{n}\right)=1$ is satisfied for every $n \in \mathbb{N}$. However $\bar{\pi}_{\alpha}(\emptyset)=0$ holds, which completes the proof.

Considering $\underline{\pi}_{\alpha}(\cdot)$, defined according to (4.10), two results analogous to Theorem 4.14 and Theorem 4.28 hold:

Theorem 4.29 Suppose that $(\Omega, \mathcal{A}, \mathcal{P})$ is a complete probability space and that $X^{\star}: \Omega \rightarrow \mathcal{F}_{c}^{d}$ is a d-dimensional fuzzy random vector. For every $B \in \mathcal{B}\left(\mathbb{R}^{d}\right)$ and $\alpha \in(0,1]$ let $\underline{\pi}_{\alpha}(B)$ be defined according to (4.10) then:

- For every Borel set $B \in \mathcal{B}\left(\mathbb{R}^{d}\right)$ and every $\alpha \in(0,1]$ the following equality holds:

$$
\underline{\pi}_{\alpha}(B)=\sup \left\{\underline{\pi}_{\alpha}(K): K \subseteq B, K \in \mathcal{K}^{d}\right\}=\inf \left\{\underline{\pi}_{\alpha}(G): G \supseteq B, G \text { open }\right\}
$$

Proof: According to Lemma $4.22 \underline{\pi}_{\alpha}(B)=1-\bar{\pi}_{\alpha}\left(B^{c}\right)$ is satisfied for every $B \in \mathcal{B}\left(\mathbb{R}^{d}\right)$ and every $\alpha \in(0,1]$, which makes it possible to apply the properties stated in Theorem 4.27. Furthermore obviously the identity

$$
1-\inf \left\{\bar{\pi}_{\alpha}(G): G \supseteq B, G \text { open }\right\}=\sup \left\{1-\bar{\pi}_{\alpha}(G): G \supseteq B, G \text { open }\right\}
$$

holds for every $B \in \mathcal{B}\left(\mathbb{R}^{d}\right)$ and every $\alpha \in(0,1]$.

If $F \subseteq \mathbb{R}^{d}$ is a closed set and $X_{\alpha}(\omega) \subseteq F$ then there exists an integer $n \in \mathbb{N}$ such that $X_{\alpha}(\omega) \subseteq F \cap[-n, n]^{d}$ is fulfilled, which shows that

$$
\left\{\omega \in \Omega: X_{\alpha}(\omega) \subseteq F\right\}=\bigcup_{n=1}^{\infty}\left\{\omega \in \Omega: X_{\alpha}(\omega) \subseteq F \cap[-n, n]^{d}\right\}
$$

holds for every closed set $F$ and every $\alpha \in(0,1]$. Since $F \cap[-n, n]^{d}$ is compact this yields that $\underline{\pi}_{\alpha}(F)=\sup \left\{\underline{\pi}_{\alpha}(K): K \subseteq F, K \in \mathcal{K}^{d}\right\}$.

Having this, suppose now that $B \in \mathcal{B}\left(\mathbb{R}^{d}\right)$, then the first part of the identity stated in the theorem follows directly from:

$$
\begin{aligned}
\underline{\pi}_{\alpha}(B) & =1-\bar{\pi}_{\alpha}\left(B^{c}\right)=1-\inf \left\{\bar{\pi}_{\alpha}(G): G \supseteq B^{c}, G \text { open }\right\} \\
& =\sup \left\{1-\bar{\pi}_{\alpha}(G): G \supseteq B^{c}, G \text { open }\right\} \\
& =\sup \left\{\underline{\pi}_{\alpha}(F): F \subseteq B, F \text { closed }\right\} \\
& =\sup \left\{\underline{\pi}_{\alpha}(K): K \subseteq B, K \in \mathcal{K}^{d}\right\}
\end{aligned}
$$

The second part of the identity can be proved similarly. 
Theorem 4.30 Suppose that $(\Omega, \mathcal{A}, \mathcal{P})$ is a complete probability space and that $X^{\star}: \Omega \rightarrow \mathcal{F}_{c}^{d}$ is a d-dimensional fuzzy random vector. For every $B \in \mathcal{B}\left(\mathbb{R}^{d}\right)$ and $\alpha \in(0,1]$ let $\underline{\pi}_{\alpha}(B)$ be defined according to (4.10) then:

- The set function $\pi_{\alpha}(\cdot)$ is continuous from above, i.e. if $\left(B_{n}\right)_{n \in \mathbb{N}}$ is a monotonically decreasing sequence of Borel sets with limit $B$, then $\lim _{n \rightarrow \infty} \underline{\pi}_{\alpha}\left(B_{n}\right)=\underline{\pi}_{\alpha}(B)$ holds.

- The set function $\underline{\pi}_{\alpha}(\cdot)$ is continuous from below for open sets, i.e. if $\left(G_{n}\right)_{n \in \mathbb{N}}$ is a monotonically increasing sequence of open sets with limit $G$, then $\lim _{n \rightarrow \infty} \underline{\pi}_{\alpha}\left(G_{n}\right)=\underline{\pi}_{\alpha}(G)$ holds.

- In general $\underline{\pi}_{\alpha}(\cdot)$ is not continuous from below.

Proof: The theorem can easily be proved by using Theorem 4.28 and by using the fact that for every Borel set $B \in \mathcal{B}\left(\mathbb{R}^{d}\right)$ and every $\alpha \in(0,1]$ the identity $\underline{\pi}_{\alpha}(B)=1-\bar{\pi}_{\alpha}\left(B^{c}\right)$ holds.

If $\left(B_{n}\right)_{n \in \mathbb{N}}$ is a decreasing sequence of Borel sets in $\mathbb{R}^{d}$ with limit $B=\bigcap_{n=1}^{\infty} B_{n}$, then obviously $\left(B_{n}^{c}\right)_{n \in \mathbb{N}}$ is an increasing sequence with limit $B^{c}=\bigcup_{n=1}^{\infty} B_{n}^{c}$. Consequently applying Theorem 4.28 yields

$$
\lim _{n \rightarrow \infty} \underline{\pi}_{\alpha}\left(B_{n}\right)=\lim _{n \rightarrow \infty}\left(1-\bar{\pi}_{\alpha}\left(B_{n}^{c}\right)\right)=1-\bar{\pi}_{\alpha}\left(B^{c}\right)=\underline{\pi}_{\alpha}(B),
$$

which proves continuity from above.

Furthermore if $\left(G_{n}\right)_{n \in \mathbb{N}}$ is an increasing sequence of open sets in $\mathbb{R}^{d}$ with limit $G=\bigcup_{n=1}^{\infty} G_{n}$ then it follows immediately that

$$
\begin{aligned}
\underline{\pi}_{\alpha}(G) & =\mathcal{P}\left(\left\{\omega \in \Omega: X_{\alpha}(\omega) \subseteq \bigcup_{n=1}^{\infty} G_{n}\right\}\right) \\
& =\lim _{n \rightarrow \infty} \mathcal{P}\left(\left\{\omega \in \Omega: X_{\alpha}(\omega) \subseteq G_{n}\right\}\right) \\
& =\lim _{n \rightarrow \infty} \underline{\pi}_{\alpha}\left(G_{n}\right) .
\end{aligned}
$$

Finally the fact that in general $\bar{\pi}_{\alpha}(\cdot)$ is not continuous from below can easily be demonstrated as follows:

Choose $X^{\star}: \Omega \rightarrow \mathcal{F}_{c}^{1}$ and $G_{n}$ as in the proof of Theorem 4.28 and simply set $F_{n}=G_{n}^{c}$. Then obviously $\bigcup_{n=1}^{\infty} F_{n}=\mathbb{R}^{d}$ and $\underline{\pi}_{\alpha}\left(F_{n}\right)=0$ is satisfied for every $n \in \mathbb{N}$. However $\underline{\pi}_{\alpha}\left(\mathbb{R}^{d}\right)=1$ holds. This completes the proof. 


\section{Chapter 5}

\section{Fuzzy stochastic processes}

\subsection{Motivation, basic definitions, and properties}

As a matter of fact in various disciplines of civil engineering and other related fields (compare [30]) measurements of (continuous physical) quantities one is interested in are more or less imprecise. By neglecting this imprecision the engineer gives away many pieces of information regarding the quantification of data.

Consider for example the time-dependent assessment of the structural reliability of a building - in the majority of the cases few parameters of interest are accessible. Nevertheless, based on time series of assessments, it is demanded to forecast the reliability (safety) of the building for a certain time in the future.

Classical deterministic structural analysis tries to describe the correlation between so-called crisp input variables like load, geometry, material-specific parameters and so-called structural responses like stress or displacements by a model $M$, described by a function $f$ (schematically depicted in Figure 5.1). In the realistic case that the measurements of the input variables are imprecise naturally the output vectors themselfes are imprecise. Various different approaches concerning this issue like for example so-called $\alpha$-level optimization can be found in [30].

If in addition the uncertainty of the input variables is described by both imprecision and stochastics, fuzzy random variables and fuzzy random vectors can be used, which results in so-called fuzzy stochastic structural analysis (again compare [30]). Not surprisingly such models are gaining more and more importance.

Finally incorporating time dependence into considerations naturally leads to 
Figure 5.1: Structural Analysis schematically

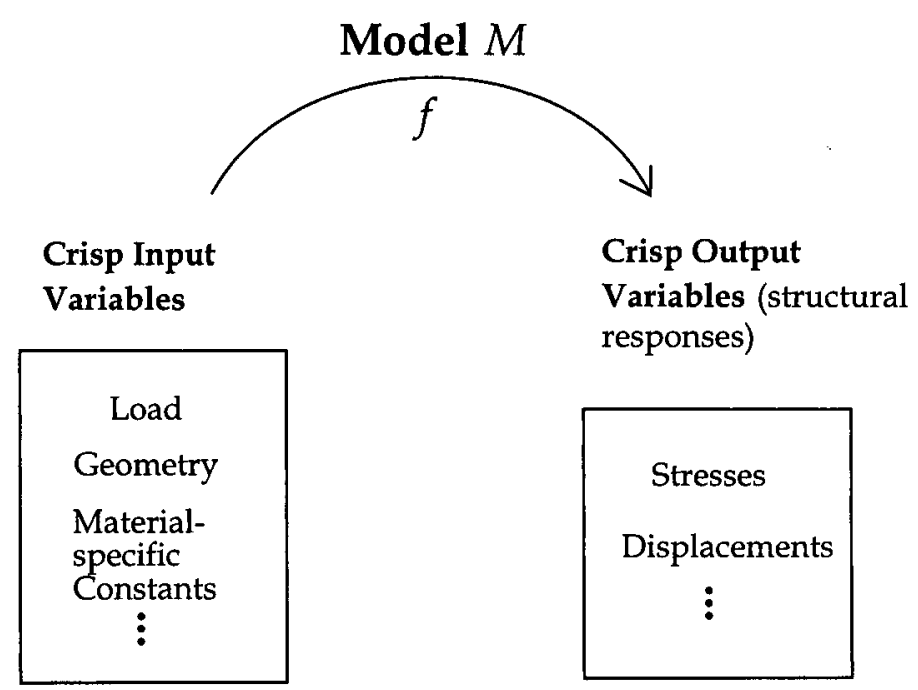

input and output variables described by fuzzy stochastic processes in discrete or continuous time (compare Definition 5.1 below).

Discrete fuzzy stochastic processes can be regarded as time series with fuzzy data (as depicted in Figure 5.2). During the last years interesting generaliza-

Figure 5.2: Time series with fuzzy data

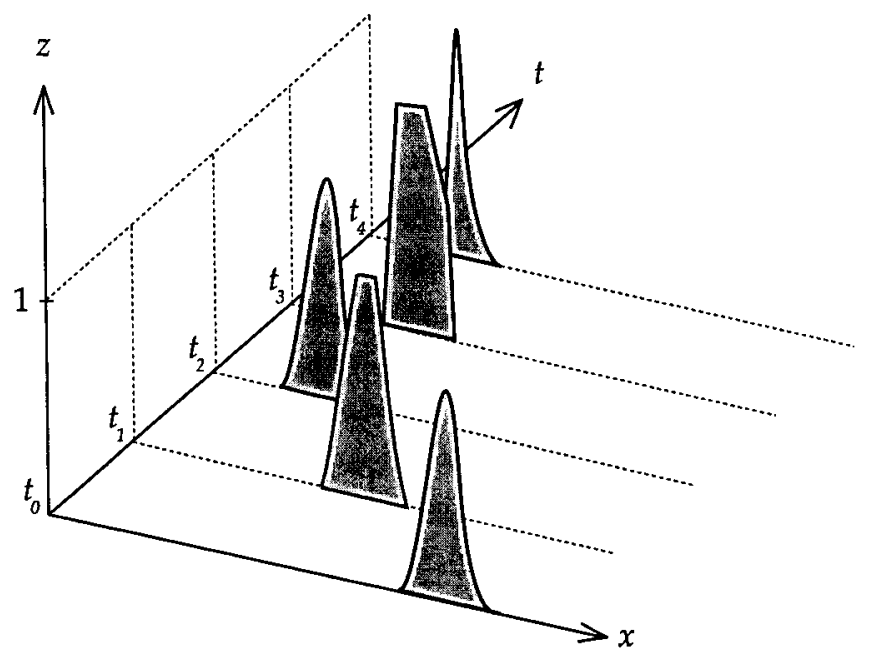


tions of techniques for classical stochastical time series with real-valued data have been generalized to the case of non-precise data (compare [15]).

These results are also applied in civil engineering, whereby mostly only subclasses of fuzzy numbers with 1-cuts consisting only of single points and socalled $\alpha$-discretization (describing a fuzzy number by finitely many $\alpha$-cuts) is used (compare [31]).

In the remaining section a general definition of fuzzy stochastic processes in continuous as well as in discrete time will be stated, separability will be defined, and, using this notion, it will be shown that similar to classical separable stochastic processes supremum, infimum, limes superior and limes inferior will be fuzzy random variables again (compare [44]).

Definition 5.1 Suppose that $(\Omega, \mathcal{A}, \mathcal{P})$ is a complete probability space and that $T \subseteq \mathbb{R}$ is an open or closed interval (possibly entire $\mathbb{R}$ ), or $T=\mathbb{N}$. Then every family $\left(X_{t}^{\star}\right)_{t \in T}$ of fuzzy random variables according to Definition 4.1 is called fuzzy stochastic process on $(\Omega, \mathcal{A}, \mathcal{P})$.

If $T=\mathbb{N}$ holds, then $\left(X_{t}^{\star}\right)_{t \in T}$ is called fuzzy stochastic process in discrete time. If $T$ is an open or closed interval then $\left(X_{t}^{\star}\right)_{t \in T}$ is called fuzzy stochastic process in continuous time.

For every $\omega \in \Omega$ the fuzzy-valued mapping $t \mapsto X_{t}^{\star}(\omega)$ is called fuzzy path of the process $\left(X_{t}^{\star}\right)_{t \in T}$.

Remark: Note that every fuzzy stochastic process in discrete time is nothing else but a sequence of fuzzy random variables.

It is well known from measure theory that given a pointwise bounded sequence of random variables $\left(X_{n}\right)_{n \in \mathbb{N}}$ (i.e. a stochastic process in discrete time)

$$
\begin{gathered}
\sup _{n \in \mathbb{N}} X_{n}, \inf _{n \in \mathbb{N}} X_{n} \text {, as well as } \\
\limsup _{n \rightarrow \infty} X_{n}:=\inf _{n \in \mathbb{N}} \sup \left\{X_{n}, X_{n+1}, \cdots\right\} \text { and } \\
\liminf _{n \rightarrow \infty} X_{n}:=\sup _{n \in \mathbb{N}} \inf \left\{X_{n}, X_{n+1}, \cdots\right\}
\end{gathered}
$$

are random variables again (compare [2], [13]). A similar result holds for stochastic processes in continuous time if the process is separable (compare [11], [24]).

It was shown by Wang and Zhang [44] that similar results hold for fuzzy stochastic processes in discrete time $\left(X_{t}^{\star}\right)_{t \in \mathbb{N}}$ and fuzzy stochastic processes in continuous time $\left(X_{t}^{\star}\right)_{t \in T}$ respectively. Unfortunately some of the results stated in [44] are simply wrong (see Example 5.3 and the subsequent remark) 
- nevertheless the main results (which are frequently used by engineers) remain true albeit the corresponding proofs are a bit more difficult.

First of all analogous to [44] supremum and infimum of families of fuzzy numbers will be defined and it will be shown that this definitions are compatible with the semiordering (1.20) explained at the end of Section 1.3.

Definition 5.2 Suppose that $\left(x_{i}^{\star}\right)_{i \in I}$ (I an arbitrary index set) is a family of fuzzy numbers with $\alpha$-cuts $\left[x_{i}^{\star}\right]_{\alpha}:=\left[\underline{x}_{i, \alpha}, \bar{x}_{i, \alpha}\right]$ for every $\alpha \in(0,1]$ and $i \in I$. Then $\left(x_{i}^{\star}\right)_{i \in I}$ is called cut-wise bounded if

$$
\left[\inf _{i \in I} \underline{x}_{i, \alpha}, \sup _{i \in I} \bar{x}_{i, \alpha}\right] \in(-\infty, \infty)
$$

holds for every $\alpha \in(0,1]$.

Given a cut-wise bounded family $\left(x_{i}^{\star}\right)_{i \in I}$ of fuzzy numbers at first sight it seems natural to define the supremum $\sup _{i \in I}\left(x_{i}^{\star}\right)$ and the infimum $\inf _{i \in I}\left(x_{i}^{\star}\right)$ of the family $\left(x_{i}^{\star}\right)_{i \in I}$ as those fuzzy numbers with $\alpha$-cuts

$$
\left[\sup _{i \in I} \underline{x}_{i, \alpha}, \sup _{i \in I} \bar{x}_{i, \alpha}\right] \quad \text { and } \quad\left[\inf _{i \in I} \underline{x}_{i, \alpha}, \inf _{i \in I} \bar{x}_{i, \alpha}\right]
$$

respectively for every $\alpha \in(0,1]$.

This surely works for finite index sets $I$, however unfortunately in general $\left(\left[\sup _{i \in I} \underline{x}_{i, \alpha}, \sup _{i \in I} \bar{x}_{i, \alpha}\right]\right)_{\alpha \in(0,1]}$ and $\left(\left[\inf _{i \in I} \underline{x}_{i, \alpha}, \inf _{i \in I} \bar{x}_{i, \alpha}\right]\right)_{\alpha \in(0,1]}$ are not families of $\alpha$-cuts of a fuzzy number as the following counter-example shows:

Example 5.3 Consider the following sequence $\left(x_{n}^{\star}\right)_{n \in \mathbb{N}}$ of fuzzy numbers defined via their $\alpha$-cuts by

$\left[x_{n}^{\star}\right]_{\alpha}:=\left\{\begin{array}{ll}{[0,1]} & \text { if } \alpha \in\left(0,2^{-1}-2^{-n}\right) \\ {\left[0,2^{n-1}-2^{n} \alpha\right]} & \text { if } \alpha \in\left[2^{-1}-2^{-n}, 2^{-1}\right] \\ \{0\} & \text { if } \alpha \in\left(2^{-1}, 1\right]\end{array}\right\} \forall \alpha \in(0,1], \forall n \in \mathbb{N}$.

Calculating the first part of (5.1) for this sequence immediately yields

$$
\left[\sup _{n \in \mathbb{N}} \underline{x}_{n, \alpha}, \sup _{n \in \mathbb{N}} \bar{x}_{n, \alpha}\right]=\left\{\begin{array}{ll}
{[0,1]} & \text { if } \alpha \in\left(0,2^{-1}\right) \\
\{0\} & \text { if } \alpha \in\left[2^{-1}, 1\right]
\end{array}\right\} \forall \alpha \in(0,1],
$$

which according to Theorem 1.4 is not a family of $\alpha$-cuts of a fuzzy number since

$$
\bigcap_{\alpha<1 / 2}\left[\sup _{n \in \mathbb{N}} \underline{x}_{n, \alpha}, \sup _{n \in \mathbb{N}} \bar{x}_{n, \alpha}\right]=[0,1] \neq\{0\}
$$

holds. An example falsifying that $\left(\left[\inf _{i \in I} \underline{x}_{i, \alpha}, \inf _{i \in I} \bar{x}_{i, \alpha}\right]\right)_{\alpha \in(0,1]}$ is a family of $\alpha$-cuts of a fuzzy number can be constructed analogously. 
Remark: Note that in [44] it is stated that $\left(\left[\sup _{i \in I} \underline{x}_{i, \alpha}, \sup _{i \in I} \bar{x}_{i, \alpha}\right]\right)_{\alpha \in(0,1]}$ and $\left(\left[\inf _{i \in I} \underline{x}_{i, \alpha}, \inf _{i \in I} \bar{x}_{i, \alpha}\right]\right)_{\alpha \in(0,1]}$ are families of $\alpha$-cuts of a fuzzy number, and that this is an essential result which is used throughout their paper.

However this is falsified by Example 5.3.

Despite the fact that for a cut-wise bounded family $\left(x_{i}^{\star}\right)_{i \in I}$ in general the families $\left(\left[\sup _{i \in I} \underline{x}_{i, \alpha}, \sup _{i \in I} \bar{x}_{i, \alpha}\right]\right)_{\alpha \in(0,1]}$ and $\left(\left[\inf _{i \in I} \underline{x}_{i, \alpha}, \inf _{i \in I} \bar{x}_{i, \alpha}\right]\right)_{\alpha \in(0,1]}$ are not families of $\alpha$-cuts of a fuzzy number, both families are nested monotonically decreasing families of compact non-empty intervals in $\alpha$. Consequently according to Theorem 1.5 and Theorem 1.36 there exist fuzzy numbers (in fact the convex hulls), which will be denoted by $\sup _{i \in I} x_{i}^{\star}$ and $\inf _{i \in I} x_{i}^{\star}$ respectively, such that

$$
\left[\sup _{i \in I} x_{i}^{\star}\right]_{\alpha}=\left[\sup _{i \in I} \underline{x}_{i, \alpha}, \sup _{i \in I} \bar{x}_{i, \alpha}\right]
$$

and

$$
\left[\inf _{i \in I} x_{i}^{\star}\right]_{\alpha}=\left[\inf _{i \in I} x_{i, \alpha}, \inf _{i \in I} \bar{x}_{i, \alpha}\right]
$$

holds for $\lambda$-almost every $\alpha \in(0,1]$ (following the proof of Theorem 1.36 in fact (5.3) and (5.4) even hold outside a countable subset of $(0,1])$.

Definition 5.4 Given a cut-wise bounded family $\left(x_{i}^{\star}\right)_{i \in I}$ of fuzzy numbers throughout the rest of the thesis the supremum $\sup _{i \in I} x_{i}^{\star}$ and the infimum $\inf _{i \in I} x_{i}^{\star}$ are defined as the convex hulls of the families

$$
\left(\left[\sup _{i \in I} \underline{x}_{i, \alpha}, \sup _{i \in I} \bar{x}_{i, \alpha}\right]\right)_{\alpha \in(0,1]} \quad \text { and } \quad\left(\left[\inf _{i \in I} \underline{x}_{i, \alpha}, \inf _{i \in I} \bar{x}_{i, \alpha}\right]\right)_{\alpha \in(0,1]}
$$

respectively (compare Theorem 1.5 and Theorem 1.36).

The following lemma shows that this definition of the supremum and infimum is compatible with $\preceq$ in $(1.20)$ :

Lemma 5.5 Suppose that $\left(x_{i}^{\star}\right)_{i \in I}$ is a cut-wise bounded family of fuzzy numbers, let the supremum $\sup _{i \in I} x_{i}^{\star} \in \mathcal{F}_{c}^{1}$ and the infimum $\inf _{i \in I} x_{i}^{\star} \in \mathcal{F}_{c}^{1}$ be defined according to Definition 5.4. Then $\sup _{i \in I} x_{i}^{\star}$ is the least upper bound and $\inf _{i \in I} x_{i}^{\star}$ is the greatest lower bound of the family $\left(x_{i}^{\star}\right)_{i \in I}$ with respect to the semiordering $\preceq$ in (1.20).

Proof: Set $s^{\star}:=\sup _{i \in I} x_{i}^{\star} \in \mathcal{F}_{c}^{1}$ and $\left[s^{\star}\right]_{\alpha}=:\left[\underline{s}_{\alpha}, \bar{s}_{\alpha}\right]$ for every $\alpha \in(0,1]$, then it follows from the proof of Theorem 1.36 that

$$
\left[s^{\star}\right]_{\alpha}=\left[\underline{s}_{\alpha}, \bar{s}_{\alpha}\right] \supseteq\left[\sup _{i \in I} \underline{x}_{i, \alpha}, \sup _{i \in I} \bar{x}_{i, \alpha}\right]
$$


holds for all $\alpha \in(0,1]$. Suppose that $\Lambda \subseteq(0,1]$ is the set for which equality holds, then again according to the proof of Theorem $1.36(0,1] \backslash \Lambda$ is a countable set.

Because of (5.5) $\sup _{i \in I} \bar{x}_{i, \alpha} \leq \bar{s}_{\alpha}$ holds for every $\alpha \in(0,1]$. Since $\underline{s}_{\alpha}$, as a function of $\alpha$, is monotonically increasing and left-continuous in $\alpha$ (compare Theorem 1.4 ), it follows that for every $\beta \in(0,1] \backslash \Lambda$ and every monotonically increasing sequence $\left(\alpha_{k}\right)_{k \in \mathbb{N}}$ in $\Lambda$, that converges to $\beta, \lim _{k \rightarrow \infty} \underline{s}_{\alpha_{k}}=\underline{s}_{\beta}$ holds. Because of $\underline{s}_{\beta} \geq \underline{s}_{\alpha_{k}}=\sup _{i \in I} \underline{x}_{i, \alpha_{k}}$ and the fact that $\lim _{k \rightarrow \infty} \underline{x}_{i, \alpha_{k}}=\underline{x}_{i, \beta}$ for every $i \in I$ it follows immediately that $\underline{s}_{\beta} \geq \underline{x}_{i, \beta}$ holds for every $i \in I$.

This shows that $\underline{s}_{\beta} \geq \sup _{i \in I} \underline{x}_{i, \beta}$ is satisfied for every $\beta \in(0,1] \backslash \Lambda$. Since $\underline{s}_{\beta} \geq$ $\sup _{i \in I} \underline{x}_{i, \beta}$ surely holds for every $\beta \in \Lambda$, this completes the proof that $\sup _{i \in I} x_{i}^{\star}$ is an upper bound of the family $\left(x_{i}^{\star}\right)_{i \in I}$ with respect to the semiordering (1.20), i.e.

$$
x_{j}^{\star} \preceq \sup _{i \in I} x_{i}^{\star}=s^{\star}
$$

holds for every $j \in I$.

It remains to show that $\sup _{i \in I} x_{i}^{\star}$ is the least upper bound with respect to the semiordering $\preceq$. Suppose that $u^{\star} \in \mathcal{F}_{c}^{1}$ is another upper bound with respect to $\preceq$ and denote by $\left[\underline{u}_{\alpha}, \bar{u}_{\alpha}\right]$ the $\alpha$-cuts of $u^{\star}$. Then it follows immediately from (1.20) that $\left[\sup _{i \in I} \underline{x}_{i, \alpha}, \sup _{i \in I} \bar{x}_{i, \alpha}\right] \preceq\left[\underline{u}_{\alpha}, \bar{u}_{\alpha}\right]$ holds for every $\alpha \in(0,1]$, which implies that

$$
\left[\underline{s}_{\alpha}, \bar{s}_{\alpha}\right] \preceq\left[\underline{u}_{\alpha}, \bar{u}_{\alpha}\right] \text { for every } \alpha \in \Lambda .
$$

Since both $s^{\star}$ and $u^{\star}$ are fuzzy numbers, it follows from Theorem 1.4 that $\left[\underline{s}_{\alpha}, \bar{s}_{\alpha}\right] \preceq\left[\underline{u}_{\alpha}, \bar{u}_{\alpha}\right]$ also holds for every $\alpha \in(0,1] \backslash \Lambda$, which shows that $s^{\star} \preceq u^{\star}$, and therefore completes the proof that $s^{\star}=\sup _{i \in I} x_{i}^{\star}$ is the least upper bound of the family $\left(x_{i}^{\star}\right)_{i \in I}$.

The fact that $\inf _{i \in I} x_{i}^{\star}$ is the greatest lower bound of the family $\left(x_{i}^{\star}\right)_{i \in I}$ with respect to the semiordering $\preceq$ can be proved analogously.

Definition 5.6 Given a cut-wise bounded sequence $\left(x_{n}^{\star}\right)_{n \in \mathbb{N}}$ of fuzzy numbers in the sequel the fuzzy numbers limes superior lim $\sup _{n \rightarrow \infty} x_{n}^{\star}$ and the limes inferior $\liminf _{n \rightarrow \infty} x_{n}^{\star}$, based on Definition 5.4 , are defined by

$$
\begin{aligned}
\limsup _{n \rightarrow \infty} x_{n}^{\star} & :=\inf _{n \in \mathbb{N}} \sup \left\{x_{n}^{\star}, x_{n+1}^{\star}, \cdots\right\} \quad \text { and } \\
\liminf _{n \rightarrow \infty} x_{n}^{\star} & :=\sup _{n \in \mathbb{N}} \inf \left\{x_{n}^{\star}, x_{n+1}^{\star}, \cdots\right\}
\end{aligned}
$$

respectively.

Remark: Note that the fact that $\lim _{\sup _{n \rightarrow \infty}} x_{n}^{\star}$ and $\liminf _{n \rightarrow \infty} x_{n}^{\star}$ are fuzzy numbers follows immediately from the construction. 
The next lemma shows that one could also define the limes superior and the limes inferior directly by starting from classical limes superior and the limes inferior of the boundaries $\underline{x}_{n, \alpha}$ and $\bar{x}_{n, \alpha}$ of the $\alpha$-cuts $\left[x_{n}^{\star}\right]_{\alpha}$.

Lemma 5.7 Suppose that $\left(x_{n}^{\star}\right)_{i \in \mathbb{N}}$ is a cut-wise bounded sequence of fuzzy numbers and let limsup $\sup _{n \rightarrow \infty} x_{n}^{\star}$ and $\liminf _{n \rightarrow \infty} x_{n}^{\star}$ be defined according to Definition 5.6. Then for $\lambda$-almost every $\alpha \in(0,1]$ the following two equalities hold:

$$
\begin{aligned}
{\left[\limsup _{n \rightarrow \infty} x_{n}^{\star}\right]_{\alpha} } & =\left[\limsup _{n \rightarrow \infty} \underline{x}_{n, \alpha}, \limsup _{n \rightarrow \infty} \bar{x}_{n, \alpha}\right] \\
{\left[\liminf _{n \rightarrow \infty} x_{n}^{\star}\right]_{\alpha} } & =\left[\liminf _{n \rightarrow \infty} \underline{x}_{n, \alpha}, \liminf _{n \rightarrow \infty} \bar{x}_{n, \alpha}\right]
\end{aligned}
$$

Proof: For every $n \in \mathbb{N}$ set $z_{n}^{\star}:=\sup \left\{x_{n}^{\star}, x_{n+1}^{\star}, \cdots\right\}$ and $y^{\star}:=\lim \sup _{n \rightarrow \infty} x_{n}^{\star}$. According to Theorem 1.36 for every $n \in \mathbb{N}$ there exists a countable set $N_{n} \subseteq(0,1]$ such that

$$
\left[\underline{z}_{n, \alpha}, \underline{z}_{n, \alpha}\right]:=\left[z_{n}^{\star}\right]_{\alpha}=\left[\sup _{k \geq n} \underline{x}_{k, \alpha}, \sup _{k \geq n} \bar{x}_{k, \alpha}\right]
$$

holds for every $\alpha \in N_{n}^{c}$. In the same manner (also according to Theorem 1.36) there exists a countable set $N$, such that

$$
\left[y^{\star}\right]_{\alpha}=\left[\inf _{n \in \mathbb{N}} \underline{z}_{n, \alpha}, \inf _{n \in \mathbb{N}} \bar{z}_{n, \alpha}\right]
$$

holds for every $\alpha \in N^{c}$. Define $\Lambda:=(0,1] \cap N^{c} \cap \bigcap_{n=1}^{\infty} N_{n}^{c}$, then obviously $\Lambda$ is measurable and fulfills $\lambda(\Lambda)=1$. For every $\alpha \in \Lambda$ it follows that

$$
\left[y^{\star}\right]_{\alpha}=\left[\inf _{n \in \mathbb{N}} \underline{z}_{n, \alpha}, \inf _{n \in \mathbb{N}} \bar{z}_{n, \alpha}\right]=\left[\inf _{n \in \mathbb{N}} \sup _{k \geq n} \underline{x}_{k, \alpha}, \inf _{n \in \mathbb{N}} \sup _{k \geq n} \bar{x}_{k, \alpha}\right] .
$$

This completes the proof of the first part of (5.7). The second part can be proved in the same manner.

Suppose now that $\left(X_{n}^{\star}\right)_{n \in \mathbb{N}}$ is a sequence of fuzzy random variables on a complete probability space $(\Omega, \mathcal{A}, \mathcal{P})$, such that for every $\omega \in \Omega$ the sequence $\left(X_{n}^{\star}(\omega)\right)_{n \in \mathbb{N}}$ is cut-wise bounded. Using Definition 5.4 and Definition 5.6 the supremum, the infimum, the limes superior and the limes inferior of the sequence $\left(X_{n}^{\star}\right)_{n \in \mathbb{N}}$ can be defined (pointwise) for every $\omega \in \Omega$ by:

$$
\begin{aligned}
\left(\sup _{n \in \mathbb{N}} X_{n}^{\star}\right)(\omega) & :=\sup _{n \in \mathbb{N}}\left(X_{n}^{\star}(\omega)\right) \\
\left(\inf _{n \in \mathbb{N}} X_{n}^{\star}\right)(\omega) & :=\inf _{n \in \mathbb{N}}\left(X_{n}^{\star}(\omega)\right) \\
\left(\limsup _{n \rightarrow \infty} X_{n}^{\star}\right)(\omega) & :=\limsup _{n \rightarrow \infty}\left(X_{n}^{\star}(\omega)\right) \\
\left(\liminf _{n \rightarrow \infty} X_{n}^{\star}\right)(\omega) & :=\liminf _{n \rightarrow \infty}\left(X_{n}^{\star}(\omega)\right)
\end{aligned}
$$


Obviously every mapping $\sup _{n \in \mathbb{N}} X_{n}^{\star}, \inf _{n \in \mathbb{N}} X_{n}^{\star}, \limsup _{n \in \mathbb{N}} X_{n}^{\star}$, as well as $\liminf _{n \in \mathbb{N}} X_{n}^{\star}$ is a fuzzy-valued mapping on $(\Omega, \mathcal{A}, \mathcal{P})$ by construction. In addition (and that is the interesting point) each of these four mappings is a fuzzy random variable according to Definition 4.1. This is the content of the next theorem.

Theorem 5.8 Suppose that $(\Omega, \mathcal{A}, \mathcal{P})$ is a complete probability space, and that $\left(X_{n}^{\star}\right)_{n \in \mathbb{N}}$ is a fuzzy stochastic process in discrete time (i.e. a sequence of fuzzy random variables), such that for every $\omega \in \Omega$ the sequence $\left(X_{n}^{\star}(\omega)\right)_{n \in \mathbb{N}}$ is cut-wise bounded according to Definition 5.2.

Then $\sup _{n \in \mathbb{N}} X_{n}^{\star}, \inf _{n \in \mathbb{N}} X_{n}^{\star}, \lim \sup _{n \rightarrow \infty} X_{n}^{\star}$, and $\lim \inf _{n \rightarrow \infty} X_{n}^{\star}$ defined according to (5.8) are fuzzy random variables on $(\Omega, \mathcal{A}, \mathcal{P})$.

Proof: First of all consider $S^{\star}:=\sup _{n \in \mathbb{N}} X_{n}^{\star}$, which is a fuzzy-valued mapping on $\Omega$. It follows from the construction of the convex hull, compare Theorem 1.5 and Theorem 1.36, and from Definition 5.4, that

$$
\left[\underline{S}_{\alpha}(\omega), \bar{S}_{\alpha}(\omega)\right]:=\left[S^{\star}(\omega)\right]_{\alpha}=\bigcap_{\beta<\alpha}\left[\sup _{n \in \mathbb{N}} \underline{X}_{n, \beta}(\omega), \sup _{n \in \mathbb{N}} \bar{X}_{n, \beta}(\omega)\right]
$$

holds for every $\alpha \in(0,1]$ and every $\omega \in \Omega$. Fix $\alpha \in(0,1]$ and suppose that $\left(\alpha_{k}\right)_{k \in \mathbb{N}}$ is a strictly increasing sequence in $(0, \alpha)$ that converges to $\alpha$. Then obviously

$$
\bigcap_{\beta<\alpha}\left[\sup _{n \in \mathbb{N}} \underline{X}_{n, \beta}(\omega), \sup _{n \in \mathbb{N}} \bar{X}_{n, \beta}(\omega)\right]=\bigcap_{k=1}^{\infty}\left[\sup _{n \in \mathbb{N}} \underline{X}_{n, \alpha_{k}}(\omega), \sup _{n \in \mathbb{N}} \bar{X}_{n, \alpha_{k}}(\omega)\right]
$$

holds, which implies that

$$
\left[\underline{S}_{\alpha}(\omega), \bar{S}_{\alpha}(\omega)\right]=\bigcap_{k=1}^{\infty}\left[\sup _{n \in \mathbb{N}} \underline{X}_{n, \alpha_{k}}(\omega), \sup _{n \in \mathbb{N}} \bar{X}_{n, \alpha_{k}}(\omega)\right]
$$

is satisfied for every $\omega \in \Omega$. Consequently if follows immediately (for example by applying Lemma 1.11) that

$$
\begin{aligned}
& \bar{S}_{\alpha}(\omega)=\lim _{k \rightarrow \infty}\left(\sup _{n \in \mathbb{N}}\left(\bar{X}_{n, \alpha_{k}}(\omega)\right)\right) \\
& \underline{S}_{\alpha}(\omega)=\lim _{k \rightarrow \infty}\left(\sup _{n \in \mathbb{N}}\left(\underline{X}_{n, \alpha_{k}}(\omega)\right)\right) \quad \text { and }
\end{aligned}
$$

holds for every $\omega \in \Omega$. Using the well-known fact that the supremum of every (pointwise) bounded sequence of real-valued random variables is itself a realvalued random variable (compare [2], [13]) it follows that $\underline{S}_{\alpha}$ and $\bar{S}_{\alpha}$ are 
(pointwise) limits of measurable functions according to (5.14) and therefore measurable too. Since $\alpha \in(0,1]$ was arbitrary it follows directly by applying Theorem 4.12 that $S^{\star}=\sup _{n \in \mathbb{N}} X_{n}^{\star}$ is a fuzzy random variable.

The assertion that $\inf _{n \in \mathbb{N}} X_{n}^{\star}$ is a fuzzy random variable can be proved completely analogous.

Having this and looking back at Definition 5.6 and (5.8) it follows immediately that $\lim \sup _{n \in \mathbb{N}} X_{n}^{\star}$, and $\lim \inf _{n \in \mathbb{N}} X_{n}^{\star}$ are fuzzy random variables too.

Given these results for fuzzy stochastic processes in discrete time it is possible to prove similar results for so-called separable fuzzy stochastic processes in continuous time.

Before giving an exact definition of a separable fuzzy stochastic process in continuous time the classical case of (real-valued) separable stochastic processes in continuous time and some basic properties are briefly discussed.

Remember the following definition of a (real-valued) separable stochastic process in continuous time (compare [11] and [24]). Thereby $\operatorname{cls}(A)$ as before denotes the closure of the set $A$.

Definition 5.9 Suppose that $(\Omega, \mathcal{A}, \mathcal{P})$ is a complete probability space, and that $\left(X_{t}\right)_{t \in T}$ is a (real-valued) stochastic process in continuous time (i.e. $T$ is an open or closed interval). Then $\left(X_{t}\right)_{t \in T}$ is called separable, if there exists a countable set $D$ which is dense in $T$, and a set $N \in \mathcal{A}$, fulfilling $\mathcal{P}(N)=0$, such that for every $t_{0} \in T$ and every $\epsilon>0$

$$
X_{t_{0}}(\omega) \in \operatorname{cls}\left\{X_{s}(\omega): s \in D \cap\left(t_{0}-\epsilon, t_{0}+\epsilon\right)\right\}
$$

holds for every $\omega \in N^{c}$.

Remark: Note that for a general stochastic processes in continuous time $\left(X_{t}\right)_{t \in T}$ neither $\sup _{t \in T} X_{t}$ nor $\inf _{t \in T} X_{t}$ need to be measurable, however measurability can be proved for separable stochastic processes, which is a proposition of the following lemma (again compare [11]).

Furthermore note that assuming that a process is separable is not a strong restriction, compare [11].

Lemma 5.10 Suppose that $(\Omega, \mathcal{A}, \mathcal{P})$ is a complete probability space, and that $\left(X_{t}\right)_{t \in T}$ is a separable stochastic process in continuous time with corresponding sets $D$ and $N$. Then for every open interval $I \subseteq T$ and every $\omega \in N^{c}$ the following equalities hold:

$$
\sup _{t \in I} X_{t}(\omega)=\sup _{t \in D \cap I} X_{t}(\omega), \quad \inf _{t \in I} X_{t}(\omega)=\inf _{t \in D \cap I} X_{t}(\omega)
$$


Proof: First of all notice that obviously $\sup _{t \in I} X_{t}(\omega) \geq \sup _{t \in D \cap I} X_{t}(\omega)$ holds for every $\omega \in \Omega$. In order to prove the opposite inequality suppose that $t \in I$. Since $t$ is an inner point of $I$ (open) there exists $\epsilon>0$ such that $(t-\epsilon, t+\epsilon) \subseteq I$. Separability of $\left(X_{t}\right)_{t \in T}$ implies that for every $\omega \in N^{c}$

$$
X_{t}(\omega) \in \operatorname{cls}\left\{X_{s}(\omega): s \in D \cap(t-\epsilon, t+\epsilon)\right\}
$$

holds, from which it follows immediately that

$$
\sup _{s \in D \cap(t-\epsilon, t+\epsilon)} X_{s}(\omega) \geq X_{t}(\omega)
$$

Since $t \in I$ was arbitrary this proves the first part of equation 5.11 for every $\omega \in N^{c}$. The second part can be proved similarly.

Again suppose that $(\Omega, \mathcal{A}, \mathcal{P})$ is a complete probability space, and that $\left(X_{t}\right)_{t \in T}$ is a separable stochastic process in continuous time. Remember the following definition of the limes superior of $\left(X_{t}(\omega)\right)_{t \in T}$ at $t_{0}$ and the limes inferior of $\left(X_{t}(\omega)\right)_{t \in T}$ at $t_{0} \in T$ for every $\omega \in \Omega$ :

$$
\begin{aligned}
\limsup _{t \rightarrow t_{0}} X_{t}(\omega):= & \inf _{n \in \mathbb{N}} \sup \left\{X_{s}(\omega): s \in T \cap\left(t_{0}-1 / n, t_{0}+1 / n\right)\right\} \\
\liminf _{t \rightarrow t_{0}} X_{t}(\omega):= & \sup _{n \in \mathbb{N}} \inf \left\{X_{s}(\omega): s \in T \cap\left(t_{0}-1 / n, t_{0}+1 / n\right)\right\}
\end{aligned}
$$

Proposition 5.11 Suppose that $(\Omega, \mathcal{A}, \mathcal{P})$ is a complete probability space, and that $\left(X_{t}\right)_{t \in T}$ is a (real-valued) separable stochastic process in continuous time. Furthermore suppose that $\left[\inf _{t \in T} X_{t}(\omega), \sup _{t \in T} X_{t}(\omega)\right] \subset(-\infty, \infty)$ for every $\omega \in \Omega$. Then for every open interval $I \subseteq T$ both $\sup _{t \in I} X_{t}: \Omega \rightarrow \mathbb{R}$ and $\inf _{t \in I} X_{t}: \Omega \rightarrow \mathbb{R}$ are random variables (i.e measurable).

Furthermore for every $t_{0} \in I$ both mappings limsup $\operatorname{sut}_{t \rightarrow t_{0}} X_{t}: \Omega \rightarrow \mathbb{R}$ and $\liminf _{t \rightarrow t_{0}} X_{t}: \Omega \rightarrow \mathbb{R}$, defined according to (5.12), are random variables.

Proof: For every $\omega \in \Omega$ set $Y(\omega):=\sup _{t \in I} X_{t}(\omega)<\infty$. Since $(\Omega, \mathcal{A}, \mathcal{P})$ is complete, for every $c \in \mathbb{R}$ it follows immediately from Lemma 5.10 that ( $D$ and $N$ as in Definition 5.9)

$$
\begin{aligned}
Y^{-1}((-\infty, c]) & =\left\{\omega \in \Omega: \sup _{t \in I} X_{t}(\omega) \leq c\right\} \\
& =\left\{\omega \in N: \sup _{t \in I} X_{t}(\omega) \leq c\right\} \cup\left\{\omega \in N^{c}: \sup _{t \in I} X_{t}(\omega) \leq c\right\} \\
& =\left\{\omega \in N: \sup _{t \in I} X_{t}(\omega) \leq c\right\} \cup\left\{\omega \in N^{c}: \sup _{t \in D \cap I} X_{t}(\omega) \leq c\right\} \\
& =\underbrace{\left\{\omega \in N: \sup _{t \in I} X_{t}(\omega) \leq c\right\}}_{\in \mathcal{A}} \cup \underbrace{\left(N^{c} \cap \bigcap_{t \in D \cap I} X_{t}^{-1}((-\infty, c])\right)}_{\in \mathcal{A}} .
\end{aligned}
$$


Since the system $\mathfrak{E}_{1}=\{(-\infty, c]: c \in \mathbb{R}\}$ is a generator of the Borel sets $\mathcal{B}(\mathbb{R})$ (compare [2], [13]) this shows that $Y$ is measurable, i.e. a random variable. Measurability of $\inf _{t \in I} X_{t}$ can be proved in completely the same manner.

It remains to prove the measurability of $\limsup _{t \rightarrow t_{0}} X_{t}$ and $\liminf t_{t \rightarrow t_{0}} X_{t}$, which can easily be done as follows:

Since $I$ is open, there exists $n_{0} \in \mathbb{N}$ such that $\left(t_{0}-1 / n, t_{0}+1 / n\right) \subseteq I \subseteq T$ holds for every $n \geq n_{0}$. Consequently it follows from the first part of the proof that for every $n \geq n_{0}$ the mapping $Y_{n}: \Omega \rightarrow \mathbb{R}$, defined by

$$
Y_{n}(\omega):=\sup \left\{X_{s}(\omega): s \in T \cap\left(t_{0}-1 / n, t_{0}+1 / n\right)\right\},
$$

is measurable. Since $\lim \sup _{t \rightarrow t_{0}} X_{t}(\omega)=\inf _{n \geq n_{0}} Y_{n}$ holds measurability of $\lim \sup _{t \rightarrow t_{0}} X_{t}$ follows immediately. Measurability of $\liminf \operatorname{int}_{t \rightarrow t_{0}} X_{t}$ can be proved analogously.

The notion of separability of a stochastic process can be extended to the case of fuzzy stochastic processes in the following way:

Definition 5.12 Suppose that $(\Omega, \mathcal{A}, \mathcal{P})$ is a complete probability space and that $\left(X_{t}^{\star}\right)_{t \in T}$ is a fuzzy stochastic process in continuous time. Then $\left(X_{t}^{\star}\right)_{t \in T}$ is called separable if for every $\alpha \in(0,1]$ both (real-valued) stochastic processes $\left(\bar{X}_{t, \alpha}\right)_{t \in T}$ and $\left(\underline{X}_{t, \alpha}\right)_{t \in T}$ are separable according to Definition 5.9.

Remark: Note that Definition 5.12 only demands that for every $\alpha \in(0,1]$ there exists a countable dense subset $D_{\alpha}$ of $T$ and a set $N_{\alpha} \in \mathcal{A}$, fulfilling $\mathcal{P}\left(N_{\alpha}\right)=0$, for every $\alpha$ - it is not demanded that there exist simultaneous sets $D$ and $N$ for every $\alpha$, which would be a much stronger requirement.

Given a fuzzy stochastic process $\left(X_{t}^{\star}\right)_{t \in T}$ in continuous time, an open interval $I \subseteq T$, and a point $t_{0} \in I$, then using Definition $5.4 \lim \sup _{t \rightarrow t_{0}} X_{t}^{\star}$ and $\lim \inf _{t \rightarrow t_{0}} X_{t}^{\star}$ can be defined analogous to (5.12) by

$$
\begin{aligned}
\limsup _{t \rightarrow t_{0}} X_{t}^{\star}(\omega) & :=\inf _{n \in \mathbb{N}} \sup \left\{X_{s}^{\star}(\omega): s \in T \cap\left(t_{0}-1 / n, t_{0}+1 / n\right)\right\} \\
\liminf _{t \rightarrow t_{0}} X_{t}^{\star}(\omega) & :=\sup _{n \in \mathbb{N}} \inf \left\{X_{s}^{\star}(\omega): s \in T \cap\left(t_{0}-1 / n, t_{0}+1 / n\right)\right\}
\end{aligned}
$$

for every $\omega \in \Omega$.

Remark: Note that if $\left(X_{t}^{\star}\right)_{t \in T}$ is a real-valued stochastic process then (5.13) coincides with (5.12).

The following measurability result analogous to Proposition 5.11 can be proved (compare [44]): 
Theorem 5.13 Suppose that $(\Omega, \mathcal{A}, \mathcal{P})$ is a complete probability space, that $\left(X_{t}^{\star}\right)_{t \in T}$ is a separable fuzzy stochastic process in continuous time, and that $I \subseteq T$ is an open interval. Furthermore suppose that the family $\left(X_{t}^{\star}(\omega)\right)_{t \in I}$ is cut-wise bounded for every $\omega \in \Omega$.

Then both $\sup _{t \in I} X_{t}^{\star}: \Omega \rightarrow \mathcal{F}_{c}^{1}$ and $\inf _{t \in I} X_{t}^{\star}: \Omega \rightarrow \mathcal{F}_{c}^{1}$ are fuzzy random variables according to Definition 4.1.

Furthermore for every $t_{0} \in I$ both mappings $\lim \sup _{t \rightarrow t_{0}} X_{t}^{\star}: \Omega \rightarrow \mathcal{F}_{c}^{1}$ and $\liminf \operatorname{int}_{t \rightarrow t_{0}} X_{t}^{\star}: \Omega \rightarrow \mathcal{F}_{c}^{1}$, defined according to (5.12), are fuzzy random variables according to Definition 4.1 .

Proof: For every $\omega \in \Omega$ using Definition 5.4 set $S^{\star}(\omega):=\sup _{t \in I} X_{t}^{\star}(\omega) \in \mathcal{F}_{c}^{1}$. Fix $\alpha \in(0,1]$ and suppose that $\left(\alpha_{k}\right)_{k \in \mathbb{N}}$ is a strictly increasing sequence in $(0, \alpha)$ that converges to $\alpha$. Just as in the proof of Theorem 5.8 it follows from the construction of the convex hull (again compare Theorem 1.5 and Theorem 1.36) that

$$
\left[\underline{S}_{\alpha}(\omega), \bar{S}_{\alpha}(\omega)\right]:=\left[S^{\star}(\omega)\right]_{\alpha}=\bigcap_{k=1}^{\infty}\left[\sup _{t \in I} \underline{X}_{t, \alpha_{k}}(\omega), \sup _{t \in I} \bar{X}_{t, \alpha_{k}}(\omega)\right]
$$

holds for every $\alpha \in(0,1]$ and every $\omega \in \Omega$, which in turn shows that

$$
\begin{aligned}
& \bar{S}_{\alpha}(\omega)=\lim _{k \rightarrow \infty}\left(\sup _{t \in I}\left(\bar{X}_{t, \alpha_{k}}(\omega)\right)\right) \quad \text { and } \\
& \underline{S}_{\alpha}(\omega)=\lim _{k \rightarrow \infty}\left(\sup _{t \in I}\left(\underline{X}_{t, \alpha_{k}}(\omega)\right)\right)
\end{aligned}
$$

is satisfied for every $\omega \in \Omega$.

Because of the fact that the process $\left(X_{t}^{\star}\right)_{t \in T}$ is separable by assumption it follows that for every $\alpha_{k}$ there exists a countable set $D_{k}$ and a set $N_{k} \in \mathcal{A}$, fulfilling $\mathcal{P}\left(N_{k}\right)=0$, such that $(5.11)$ is satisfied for the process $\left(\bar{X}_{t, \alpha_{k}}\right)_{t \in I}$ and the process $\left(\underline{X}_{t, \alpha_{k}}\right)_{t \in I}$ simultaneously for every $\omega \in N_{k}^{c}$.

For every $\omega \in \Omega$ and every $k \in \mathbb{N}$ define

$$
\bar{Y}_{k}(\omega):=\sup _{t \in I} \bar{X}_{t, \alpha_{k}}(\omega) \quad \text { and } \quad \underline{Y}_{k}(\omega):=\sup _{t \in I} \underline{X}_{t, \alpha_{k}}(\omega)
$$

then according to Proposition 5.11 both $\bar{Y}_{k}, \underline{Y}_{k}: \Omega \rightarrow \mathbb{R}$ are measurable for every $k \in \mathbb{N}$. Having this using (5.14) immediately yields that $\bar{S}_{\alpha}$ and $\underline{S}_{\alpha}$ are measurable functions too.

Since $\alpha \in(0,1]$ was arbitrary this completes the proof that $S^{\star}=\sup _{t \in I} X_{t}^{\star}$ is a fuzzy random variable according to Definition 4.1 .

The fact that $\inf _{t \in I}\left(X_{t}^{\star}\right): \Omega \rightarrow \mathcal{F}_{c}^{1}$ is a fuzzy random variable according to Definition 4.1 too can be proved completely analogous. 
It remains to prove the measurability of $\lim \sup _{t \rightarrow t_{0}} X_{t}^{\star}$ and $\liminf \operatorname{int}_{t \rightarrow} X_{t}^{\star}$, which can easily be done as follows:

Since $I$ is open, there exists $n_{0} \in \mathbb{N}$ such that $\left(t_{0}-1 / n, t_{0}+1 / n\right) \subseteq I \subseteq T$ holds for every $n \geq n_{0}$. Consequently it follows from the first part of the proof that for every $n \geq n_{0}$ the mapping $Z_{n}^{\star}: \Omega \rightarrow \mathcal{F}_{c}^{1}$, defined by

$$
Z_{n}^{\star}(\omega):=\sup \left\{X_{s}^{\star}(\omega): s \in T \cap\left(t_{0}-1 / n, t_{0}+1 / n\right)\right\},
$$

is measurable. Since $\lim \sup _{t \rightarrow t_{0}} X_{t}^{\star}(\omega)=\inf _{n \geq n_{0}} Z_{n}^{\star}(\omega)$, applying Theorem 5.8 shows that lim $\sup _{t \rightarrow t_{0}} X_{t}^{\star}$ is a fuzzy random variable in the sense of Definition 4.1. The assertion for $\liminf _{t \rightarrow t_{0}} X_{t}^{\star}$ can be proved analogously.

Remark: Obviously separability of a fuzzy stochastic process in continuous time could also be defined directly, i.e. without concentrating on the corresponding $\alpha$-cuts, by using one of the metrics explained in Section 1.5.

For example for a fuzzy stochastic process $\left(X_{t}^{\star}\right)_{t \in T}$ with values in $\mathcal{F}_{c, c}^{1}$ one could use the metric $\delta_{H, \infty}^{\star}$ and say that $\left(X_{t}^{\star}\right)_{t \in T}$ is (strongly) separable if there exists a countable set $D$, dense in $T$, and a set $N \in \mathcal{A}$, fulfilling $\mathcal{P}(N)=0$, such that for every $t_{0} \in T$ and every $\epsilon>0$

$$
X_{t_{0}}^{\star}(\omega) \in \operatorname{cls}_{\infty}\left\{X_{s}^{\star}(\omega): s \in D \cap\left(t_{0}-\epsilon, t_{0}+\epsilon\right)\right\}
$$

holds for every $\omega \in N^{c}$ (thereby cls $_{\infty}$ denotes the closure with respect to the metric $\left.\delta_{H, \infty}^{\star}\right)$. Obviously this notion of separability is much stronger than Definition 5.12 .

This section will be concluded by the definition of (and a result on) strongly continuous fuzzy stochastic processes in continuous time.

Definition 5.14 Suppose that $(\Omega, \mathcal{A}, \mathcal{P})$ is a complete probability space and that $\left(X_{t}^{\star}\right)_{t \in T}$ is a fuzzy stochastic process in continuous time with values in $\mathcal{F}_{c, c}^{1}$. Then $\left(X_{t}^{\star}\right)_{t \in T}$ is called strongly continuous if there exists a set $N \in \mathcal{A}$, fulfilling $\mathcal{P}(N)=0$, such that for every $\omega \in N^{c}$ the path $t \mapsto X_{t}^{\star}(\omega) \in \mathcal{F}_{c, c}^{1}$ is a continuous mapping with respect to the metric $\delta_{H, \infty}^{\star}$.

Remark Any other metric explained in Section 1.5 can be used in order to define continuity of a fuzzy stochastic process in continuous time.

Furthermore if $\left(X_{t}^{\star}\right)_{t \in T}$ fulfills Definition 5.14 then obviously for every $w \in N^{c}$ and every $\alpha \in(0,1]$ both paths $t \mapsto \bar{X}_{t, \alpha}(\omega)$ and $t \mapsto \underline{X}_{t, \alpha}(\omega)$ are continuous. (This is a direct consequence of the definition of the metric $\delta_{H, \infty}^{\star}$.)

Analogous to the real case (compare [11]) strong continuity of a fuzzy stochastic process implies separability: 
Theorem 5.15 Suppose that $(\Omega, \mathcal{A}, \mathcal{P})$ is a complete probability space and that $\left(X_{t}^{\star}\right)_{t \in T}$ is a strongly continuous fuzzy stochastic process in continuous time with values in $\mathcal{F}_{c, c}^{1}$. Then $\left(X_{t}^{\star}\right)_{t \in T}$ is separable.

Proof: Set $D:=\mathbb{Q} \cap T$, then for every $t_{0} \in T$ and every $\epsilon>0$ it follows immediately that

$$
X_{t_{0}}^{\star}(\omega) \in \operatorname{cls}_{\infty}\left\{X_{s}^{\star}(\omega): s \in D \cap\left(t_{0}-\epsilon, t_{0}+\epsilon\right)\right\}
$$

holds for every $\omega \in N^{c}$. Using the definition of the metric $\delta_{H, \infty}^{\star}$ on $\mathcal{F}_{c, c}^{1}$, this shows that both $\left(\bar{X}_{t, \alpha}\right)_{t \in T}$ and $\left(\underline{X}_{t, \alpha}\right)_{t \in T}$ are separable (real-valued) stochastic processes in continuous time according to Definition 5.9 for every $\alpha \in(0,1]$. Looking at Definition 5.12 this completes the proof.

\subsection{Independence of fuzzy random vectors}

Definition 5.16 (Independence of fuzzy random vectors)

Suppose that $(\Omega, \mathcal{A}, \mathcal{P})$ is a complete probability space and that $X^{\star}: \Omega \rightarrow \mathcal{F}_{c}^{d}$ and $Y^{\star}: \Omega \rightarrow \mathcal{F}_{c}^{d}$ are $d$-dimensional fuzzy random vectors. Then $X^{\star}$ and $Y^{\star}$ are said to be independent if for arbitrary Borel sets $B_{1}, B_{2} \in \mathcal{B}\left(\mathbb{R}^{d}\right)$ the following equality holds for every $\alpha \in(0,1]$ :

$$
\mathcal{P}\left(X_{\alpha}(\omega) \subseteq B_{1}, Y_{\alpha}(\omega) \subseteq B_{2}\right)=\mathcal{P}\left(X_{\alpha}(\omega) \subseteq B_{1}\right) \cdot \mathcal{P}\left(Y_{\alpha}(\omega) \subseteq B_{2}\right)
$$

Analogous to the notation in Theorem 1.20 and Proposition 1.21 in Section 1.2.1 set

$$
\mathcal{K}^{B}:=\left\{K \in \mathcal{K}_{c}^{d}: K \subseteq B\right\} \quad \text { and } \quad \mathcal{K}_{B}:=\left\{K \in \mathcal{K}_{c}^{d}: K \cap B \neq \emptyset\right\}
$$

for every $B \in \mathcal{B}\left(\mathbb{R}^{d}\right)$ as well as

$$
\widetilde{\mathscr{E}}_{1}:=\left\{\mathcal{K}^{B}: B \in \mathcal{B}\left(\mathbb{R}^{d}\right)\right\}, \quad \widetilde{\mathscr{E}}_{2}:=\left\{\mathcal{K}_{B}: B \in \mathcal{B}\left(\mathbb{R}^{d}\right)\right\} .
$$

Note that $\widetilde{\mathscr{E}}_{1}$ is closed under intersection, in fact $\mathcal{K}^{B_{1}} \cap \mathcal{K}^{B_{2}}=\mathcal{K}^{B_{1} \cap B_{2}}$ holds, and that $\left(\mathcal{K}^{B}\right)^{c}=\mathcal{K}_{B^{c}}$ is satisfied. Having this it follows immediately that the generated $\sigma$-algebras $\mathcal{A}_{\sigma}\left(\mathscr{F}_{1}\right)$ and $\mathcal{A}_{\sigma}\left(\mathscr{F}_{2}\right)$ respectively coincide, which, using Proposition 1.21, implies that

$$
\mathcal{A}_{\sigma}\left(\widetilde{\mathscr{E}}_{1}\right)=\mathcal{A}_{\sigma}\left(\widetilde{\mathscr{E}}_{2}\right) \supseteq \mathcal{B}\left(\left(\mathcal{K}_{c}^{d}, \delta_{H}\right)\right)
$$

holds.

Using notation (5.17) obviously independence (5.16) can be reformulated equivalently as

$$
\mathcal{P}\left(X_{\alpha}^{-1}\left(\mathcal{K}^{B_{1}}\right) \cap Y_{\alpha}^{-1}\left(\mathcal{K}^{B_{2}}\right)\right)=\mathcal{P}\left(X_{\alpha}^{-1}\left(\mathcal{K}^{B_{1}}\right)\right) \cdot \mathcal{P}\left(Y_{\alpha}^{-1}\left(\mathcal{K}^{B_{2}}\right)\right)
$$


for all Borel sets $B_{1}, B_{2} \in \mathcal{B}\left(\mathbb{R}^{d}\right)$ and every $\alpha \in(0,1]$.

The above notion of independence has the following implications summarized in the next theorem.

Theorem 5.17 Suppose that $(\Omega, \mathcal{A}, \mathcal{P})$ is a complete probability space and that $X^{\star}: \Omega \rightarrow \mathcal{F}_{c}^{d}$ and $Y^{\star}: \Omega \rightarrow \mathcal{F}_{c}^{d}$ are d-dimensional fuzzy random vectors. If $X^{\star}$ and $Y^{\star}$ are independent according to Definition 5.16, then the following assertions hold:

1. For all sets $\mathscr{C}_{1}, \mathscr{C}_{2} \in \mathcal{A}_{\sigma}\left(\widetilde{\mathscr{E}}_{1}\right)$ the following equality is fulfilled for every $\alpha \in(0,1]$ :

$$
\mathcal{P}\left(X_{\alpha}^{-1}\left(\mathscr{C}_{1}\right) \cap Y_{\alpha}^{-1}\left(\mathscr{C}_{2}\right)\right)=\mathcal{P}\left(X_{\alpha}^{-1}\left(\mathscr{C}_{1}\right)\right) \cdot \mathcal{P}\left(Y_{\alpha}^{-1}\left(\mathscr{C}_{2}\right)\right)
$$

2. $X_{\alpha}$ and $Y_{\alpha}$ are independent as $\mathcal{K}_{c}^{d}$-valued mappings on $(\Omega, \mathcal{A}, \mathcal{P})$ with respect to the Borel $\sigma$-algebra $\mathcal{B}\left(\left(\mathcal{K}_{c}^{d}, \delta_{H}\right)\right)$ for every $\alpha \in(0,1]$.

3. For every $\alpha \in(0,1]$ and every $u \in S^{d-1}$ the support functions $s_{X_{\alpha}(\omega)}(u)$ and $s_{Y_{\alpha}(\omega)}(u)$ (as functions of $\omega$ ) are independent real-valued random variables.

Proof: Since $\widetilde{\mathscr{E}}_{1}$ is closed under intersection and generates $\mathcal{A}_{\sigma}\left(\widetilde{\mathscr{E}}_{1}\right)$, it is well known from probability theory (compare [3]) that the fulfillment of (5.20) immediately implies the fulfillment of $(5.21)$ for every pair $\mathscr{C}_{1}, \mathscr{C}_{2} \in \mathcal{A}_{\sigma}\left(\widetilde{\mathscr{E}}_{1}\right)$ and every $\alpha \in(0,1]$. This proves the first assertion.

The second assertion is an immediate consequence of the first assertion since according to (5.19) $\mathcal{A}_{\sigma}\left(\widetilde{\mathscr{E}}_{1}\right)=\mathcal{A}_{\sigma}\left(\widetilde{\mathscr{E}}_{2}\right) \supseteq \mathcal{B}\left(\left(\mathcal{K}_{c}^{d}, \delta_{H}\right)\right)$ holds.

In order to prove the third assertion remember that according to Theorem 1.24

$$
\left\|s_{K}-s_{L}\right\|_{\infty}=\delta_{H}(K, L)
$$

holds for all $K, L \in \mathcal{K}_{c}^{d}$, which implies that $\left|s_{K}(u)-s_{L}(u)\right| \leq \delta_{H}(K, L)$ for every $u \in S^{d-1}$. Therefore the mapping $\Upsilon_{u}: \mathcal{K}_{c}^{d} \rightarrow \mathbb{R}$, defined by

$$
\Upsilon_{u}(K):=s_{K}(u)
$$

for every $K \in \mathcal{K}_{c}^{d}$, is Lipschitz-continuous (with Lipschitz constant 1) and hence Borel measurable.

Because of the fact that

$$
s_{X_{\alpha}(\omega)}(u)=\Upsilon_{u} \circ X_{\alpha}(\omega) \text { and } s_{Y_{\alpha}(\omega)}(u)=\Upsilon_{u} \circ Y_{\alpha}(\omega)
$$

holds it follows again by well-known results from probability theory (compare [3]) that $s_{X_{\alpha}(\omega)}(u)$ and $s_{Y_{\alpha}(\omega)}(u)$ (as functions of $\omega$ ) are independent real-valued random variables if $X^{\star}$ and $Y^{\star}$ are independent according to Definition 5.16. 
Proposition 5.18 Suppose that $(\Omega, \mathcal{A}, \mathcal{P})$ is a complete probability space and that $X^{\star}: \Omega \rightarrow \mathcal{F}_{c}^{1}$ and $Y^{\star}: \Omega \rightarrow \mathcal{F}_{c}^{1}$ are independent fuzzy random variables. Then $\bar{X}_{\alpha}$ and $\bar{Y}_{\alpha}$ as well as $\underline{X}_{\alpha}$ and $\underline{Y}_{\alpha}$ are independent real-valued random variables.

Proof: For fuzzy random variables $X^{\star}: \Omega \rightarrow \mathcal{F}_{c}^{1}$ and $Y^{\star}: \Omega \rightarrow \mathcal{F}_{c}^{1}$ according to Example 1.22 the support function fulfills

$$
\begin{aligned}
& \bar{X}_{\alpha}(\omega)=s_{X_{\alpha}(\omega)}(1), \quad \underline{X}_{\alpha}(\omega)=-s_{X_{\alpha}(\omega)}(-1) \\
& \bar{Y}_{\alpha}(\omega)=s_{Y_{\alpha}(\omega)}(1), \quad \underline{Y}_{\alpha}(\omega)=-s_{Y_{\alpha}(\omega)}(-1) .
\end{aligned}
$$

Consequently appying Theorem 5.17 it follows immediately that $\bar{X}_{\alpha}$ and $\bar{Y}_{\alpha}$ as well as $\underline{X}_{\alpha}$ and $\underline{Y}_{\alpha}$ are independent real-valued random variables.

\subsection{A Strong Law of Large Numbers for fuzzy relative frequencies}

The purpose of this section is to prove that given a sequence $\left(X_{n}^{\star}\right)_{n \in \mathbb{N}}$ of pairwise independent, identically distributed $d$-dimensional fuzzy random vectors for every Borel set $B \in \mathcal{B}\left(\mathbb{R}^{d}\right)$ with probability one the relative frequencies $h_{n}^{\star}(B, \omega)$ converge to $\mathbb{P}^{\star}(B)$ for $n \rightarrow \infty$ even with respect to various different metrics explained in Section $1.5\left(h_{n}^{\star}(B, \omega)\right.$ defined as in Section 2.1 and $\mathbb{P}^{\star}(B)$ defined according to $\left.(4.11)\right)$.

The following definitions and lemmas are preliminary steps going in that direction.

Given a sequence $\left(X_{n}^{\star}\right)_{n \in \mathbb{N}}$ of $d$-dimensional fuzzy random vectors on a probability space $(\Omega, \mathcal{A}, \mathcal{P})$ then the lower and upper relative frequencies of level $\alpha$ for every $\omega \in \Omega$ and every $B \in \mathcal{B}\left(\mathbb{R}^{d}\right)$ analogous to Section 2.1 are defined by

$$
\underline{h}_{n, \alpha}(B, \omega):=\frac{1}{n} \#\left\{i \in\{1, \ldots, n\}:\left[X_{i}^{\star}(\omega)\right]_{\alpha} \subseteq B\right\}
$$

and

$$
\bar{h}_{n, \alpha}(B, \omega):=\frac{1}{n} \#\left\{i \in\{1, \ldots, n\}:\left[X_{i}^{\star}(\omega)\right]_{\alpha} \cap B \neq \emptyset\right\}
$$

respectively.

Moreover for every $\omega \in \Omega$ and every $B \in \mathcal{B}\left(\mathbb{R}^{d}\right)$ the fuzzy relative frequency $h_{n}^{\star}(B, \omega) \in \mathcal{F}_{c}^{1}$ analogous to Section 2.1 is defined as the convex hull of the family $\left(\left[\underline{h}_{n, \alpha}(B, \omega), \bar{h}_{n, \alpha}(B, \omega)\right]\right)_{\alpha \in(0,1]}$. 
Definition 5.19 Suppose that $(\Omega, \mathcal{A}, \mathcal{P})$ is a complete probability space, that $X^{\star}: \Omega \rightarrow \mathcal{F}_{c}^{d}$ is a d-dimensional fuzzy random vector, and that $B \in \mathcal{B}\left(\mathbb{R}^{d}\right)$ is an arbitray Borel set. Then for every $\alpha \in(0,1]$ the random variable $T_{X_{\alpha}}^{B}: \Omega \rightarrow\{0,1\}$, defined by

$$
T_{X_{\alpha}}^{B}(\omega):=\left\{\begin{array}{ll}
1 & \text { if } X_{\alpha}(\omega) \cap B \neq \emptyset \\
0 & \text { otherwise }
\end{array}\right\} \forall \omega \in \Omega,
$$

is called $\alpha$-cut hitting variable of $B$ induced by $X^{\star}$ and the random variable $I_{X_{\alpha}}^{B}: \Omega \rightarrow\{0,1\}$, defined by

$$
I_{X_{\alpha}}^{B}(\omega):=\left\{\begin{array}{ll}
1 & \text { if } X_{\alpha}(\omega) \subseteq B \\
0 & \text { otherwise }
\end{array}\right\} \forall \omega \in \Omega,
$$

is called $\alpha$-cut inclusion variable of $B$ induced by $X^{\star}$.

Remark: Throughout the whole section the notation explained in Section 4.3 will be used.

Since $X^{\star}$ is a $d$-dimensional fuzzy random vector obviously $T_{X_{\alpha}}^{B}$ and $I_{X_{\alpha}}^{B}$ are measurable for every $\alpha \in(0,1]$. Furthermore $T_{X_{\alpha}}^{B}$ and $I_{X_{\alpha}}^{B}$ are integrable for every $\alpha \in(0,1]$ and the expectations $\mathbb{E}\left(T_{X_{\alpha}}^{B}\right)$ and $\mathbb{E}\left(I_{X_{\alpha}}^{B}\right)^{\alpha}$ can be calculated as follows:

$$
\begin{gathered}
\mathbb{E}\left(T_{X_{\alpha}}^{B}\right)=\mathcal{P}\left(\left\{\omega \in \Omega: X_{\alpha}(\omega) \cap B \neq \emptyset\right\}\right)=\bar{\pi}_{\alpha}(B) \\
\mathbb{E}\left(I_{X_{\alpha}}^{B}\right)=\mathcal{P}\left(\left\{\omega \in \Omega: X_{\alpha}(\omega) \subseteq B\right\}\right)=\underline{\pi}_{\alpha}(B)
\end{gathered}
$$

Lemma 5.20 Suppose that $(\Omega, \mathcal{A}, \mathcal{P})$ is a complete probability space, that $X^{\star}, Y^{\star}: \Omega \rightarrow \mathcal{F}_{c}^{d}$ are d-dimensional fuzzy random vectors and that $B \in \mathcal{B}\left(\mathbb{R}^{d}\right)$ is an arbitray Borel set. If $X^{\star}$ and $Y^{\star}$ are independent then $T_{X_{\alpha}}^{B}$ and $T_{Y_{\alpha}}^{B}$ as well as $I_{X_{\alpha}}^{B}$ and $I_{Y_{\alpha}}^{B}$ are independent random variables for every $\alpha \in(0,1]$.

Proof: It is well known from probability theory (compare for instance [3]) that the independence of two events $E_{1}, E_{2}$ implies the independence of the generated $\sigma$-algebras $\mathcal{A}_{\sigma}\left(\left\{E_{1}\right\}\right)$ and $\mathcal{A}_{\sigma}\left(\left\{E_{2}\right\}\right)$.

Consequently if $X^{\star}$ and $Y^{\star}$ are independent according to Definition 5.16, then obviously the events $\left\{\omega \in \Omega: X_{\alpha}(\omega) \subseteq B\right\}$ and $\left\{\omega \in \Omega: Y_{\alpha}(\omega) \subseteq B\right\}$ respectively, and the corresponding generated $\sigma$-algebras are independent, which immediately shows that for every $\alpha \in(0,1] T_{X_{\alpha}}^{B}$ and $T_{Y_{\alpha}}^{B}$ as well as $I_{X_{\alpha}}^{B}$ and $I_{Y_{\alpha}}^{B}$ are independent random variables. 
Lemma 5.21 Suppose that $(\Omega, \mathcal{A}, \mathcal{P})$ is a complete probability space, that $X^{\star}, X_{1}^{\star}, X_{2}^{\star}, \cdots$ are pairwise independent, identically distributed d-dimensional fuzzy random vectors and that $B \in \mathcal{B}\left(\mathbb{R}^{d}\right)$ is an arbitrary Borel set. Futhermore for every $\alpha \in(0,1]$ let $\bar{\pi}_{\alpha}$ and $\underline{\pi}_{\alpha}$ be defined according to (4.10).

Then for every $\alpha \in(0,1]$ there exists a set $N \in \mathcal{A}$, fulfilling $\mathcal{P}(N)=0$, such that for every $\omega \in N^{c}$ the following identities hold:

$$
\begin{aligned}
& \lim _{n \rightarrow \infty} \bar{h}_{n, \alpha}(B, \omega)=\bar{\pi}_{\alpha}(B) \\
& \lim _{n \rightarrow \infty} \underline{h}_{n, \alpha}(B, \omega)=\underline{\pi}_{\alpha}(B)
\end{aligned}
$$

Proof: For fixed $B \in \mathcal{B}\left(\mathbb{R}^{d}\right)$, every $\alpha \in(0,1]$, and every $n \in \mathbb{N}$, define $T_{\left(X_{n}\right)_{\alpha}}^{B}$ and $I_{\left(X_{n}\right)_{\alpha}}^{B}$ according to $(5.24)$ and (5.25) respectively.

Suppose now that $B \in \mathcal{B}\left(\mathbb{R}^{d}\right)$ and $\alpha \in(0,1]$ are fixed. To simplify matters for every $n \in \mathbb{N}$ and every $\omega \in \Omega$ set

$$
T_{n}(\omega):=T_{\left(X_{n}\right)_{\alpha}}^{B}(\omega) \quad \text { and } \quad I_{n}(\omega):=I_{\left(X_{n}\right)_{\alpha}}^{B}(\omega) .
$$

Since by assumption $X_{1}^{\star}, X_{2}^{\star}, \cdots$ are pairwise independent Lemma 5.20 implies that both $\left(T_{n}\right)_{n \in \mathbb{N}}$ and $\left(I_{n}\right)_{n \in \mathbb{N}}$ are sequences of pairwise independent random variables. Because of the fact that obviously all $T_{n}$ and all $I_{n}$ are integrable and identically distributed the Strong Law of Large Numbers (Theorem 2.1) can be applied to both sequences, which yields the following result: There exist two sets $N_{1}, N_{2} \in \mathcal{A}$, fulfilling $\mathcal{P}\left(N_{1}\right)=\mathcal{P}\left(N_{2}\right)=0$, such that both

$$
\lim _{n \rightarrow \infty} \frac{1}{n} \sum_{i=1}^{n} T_{i}(\omega)=\lim _{n \rightarrow \infty} \bar{h}_{n, \alpha}(B, \omega)=\mathbb{E}\left(T_{X_{\alpha}}^{B}\right)=\bar{\pi}_{\alpha}(B),
$$

and

$$
\lim _{n \rightarrow \infty} \frac{1}{n} \sum_{i=1}^{n} I_{i}(\omega)=\lim _{n \rightarrow \infty} \underline{h}_{n, \alpha}(B, \omega)=\mathbb{E}\left(I_{X_{\alpha}}^{B}\right)=\underline{\pi}_{\alpha}(B),
$$

holds for every $\omega \in\left(N_{1} \cup N_{2}\right)^{c}$.

Setting $N:=N_{1} \cup N_{2}$ completes the proof since $\mathcal{P}(N)=\mathcal{P}\left(N_{1} \cup N_{2}\right)=0$.

The next main step consists in finding a simultaneous set $N \in \mathcal{A}$ of probability zero for $\lambda$-almost every $\alpha \in(0,1]$ and in passing over from $\bar{\pi}_{\alpha}$ to $\bar{p}_{\alpha}$ and from $\underline{\pi}_{\alpha}$ to $\underline{p}_{\alpha}$.

Looking back to Section 4.3 , Lemma 4.23 can be used to prove the following simple result, which will be helpful in the sequel:

Lemma 5.22 Suppose that $(\Omega, \mathcal{A}, \mathcal{P})$ is a complete probability space, that $X^{\star}$ is a d-dimensional fuzzy random vector and that $B \in \mathcal{B}\left(\mathbb{R}^{d}\right)$ is a Borel 
set. Using the notation from Section 4.3 define functions $g, \bar{g}:(0,1] \rightarrow[0,1]$ by setting $\underline{g}(\alpha):=\underline{p}_{\alpha}(B)$ and $\bar{g}(\alpha):=\bar{p}_{\alpha}(B)$ for every $\alpha \in(0,1]$, then the following assertions are fulfilled:

- If $\bar{g}$ is continuous at $\alpha_{0} \in(0,1]$ then $\bar{p}_{\alpha_{0}}(B)=\bar{\pi}_{\alpha_{0}}(B)$ holds.

- If $\underline{g}$ is continuous at $\alpha_{0} \in(0,1]$ then $\underline{p}_{\alpha_{0}}(B)=\underline{\pi}_{\alpha_{0}}(B)$ holds.

Proof: Note that it follows immediately from Lemma 1.4 and Lemma 4.23 that $\bar{g}$ is a monotonically decreasing, left-continuous function, that $g$ is a monotonically increasing, left-continuous function, and that $\bar{p}_{\alpha}(B) \geq \overline{\bar{\pi}}_{\boldsymbol{\alpha}}(B)$ as well as $\underline{p}_{\alpha}(B) \leq \underline{\pi}_{\alpha}(B)$ holds for arbitrary $\alpha \in(0,1]$.

Suppose now that $\bar{p}_{\alpha_{0}}(B)-\bar{\pi}_{\alpha_{0}}(B)=: c>0$, then

$$
\bar{p}_{\alpha}(B) \leq \bar{\pi}_{\alpha_{0}}(B)=\bar{p}_{\alpha_{0}}(B)-c
$$

follows for every $\alpha>\alpha_{0}$, which implies that $\bar{g}$ can not be continuous at $\alpha_{0}$ and therefore proves the first assertion. The second assertion can be proved similarly.

Theorem 5.23 Suppose that $(\Omega, \mathcal{A}, \mathcal{P})$ is a complete probability space, that $X^{\star}, X_{1}^{\star}, X_{2}^{\star}, \cdots$ are pairwise independent, identically distributed d-dimensional fuzzy random vectors and that $B \in \mathcal{B}\left(\mathbb{R}^{d}\right)$ is an arbitrary Borel set.

Then there exists a set $\Lambda \subseteq(0,1]$, fulfilling $\lambda(\Lambda)=1$, and a set $N \in \mathcal{A}$, fulflling $\mathcal{P}(N)=0$, such that for every $\alpha \in \Lambda$ and every $\omega \in N^{c}$ the following identities hold:

$$
\begin{aligned}
& \lim _{n \rightarrow \infty} \bar{h}_{n, \alpha}(B, \omega)=\bar{p}_{\alpha}(B) \\
& \lim _{n \rightarrow \infty} \underline{h}_{n, \alpha}(B, \omega)=\underline{p}_{\alpha}(B)
\end{aligned}
$$

Proof: Define the functions $\underline{g}, \bar{g}$ as in Lemma 5.22 and denote by $\Lambda=\mathcal{S}(\underline{g}, \bar{g})$ the set of all points $\alpha \in(0,1]$ at which both $\underline{g}$ and $\bar{g}$ are continuous. Because of the fact that $\underline{g}$ and $\bar{g}$ are monotonic bounded functions the set $\Lambda \subseteq(0,1]$ is the complement of a countable set (compare [16]), and therefore has full measure, i.e. $\lambda(\Lambda)=1$. Furthermore $\Lambda$ is (as subset of a separable set in a metric space itself) separable, so there exists a countable dense set $\Lambda_{0} \subseteq \Lambda$. Note that by construction according to Lemma $5.22 \underline{p}_{\alpha}(B)=\underline{\pi}_{\alpha}(B)$ and $\bar{p}_{\alpha}(B)=\bar{\pi}_{\alpha}(B)$ holds for every $\alpha \in \Lambda$.

Since countable unions of sets of probability zero also have probability zero, applying Lemma 5.21 yields that there exists a (simultaneous) set $N \in \mathcal{A}$ for every $\alpha \in \Lambda_{0}$, fulfilling $\mathcal{P}\left(N^{c}\right)=1$, such that

$$
\lim _{n \rightarrow \infty} \bar{h}_{n, \alpha}(B, \omega)=\bar{p}_{\alpha}(B) \quad \text { and } \quad \lim _{n \rightarrow \infty} \underline{h}_{n, \alpha}(B, \omega)=\underline{p}_{\alpha}(B)
$$


holds for every $\alpha \in \Lambda_{0}$ and every $\omega \in N^{c}$. This result will now be extended from $\Lambda_{0}$ to $\Lambda$ by using monotonicity in the following way:

For arbitrary but fixed $\omega \in N^{c}$ define functions $\underline{g}_{n}, \bar{g}_{n}:(0,1] \rightarrow[0,1]$ for every $n \in \mathbb{N}$ by setting

$$
\bar{g}_{n}(\alpha):=\bar{h}_{n, \alpha}(B, \omega) \quad \text { and } \quad \underline{g}_{n}(\alpha):=\underline{h}_{n, \alpha}(B, \omega)
$$

for every $\alpha \in(0,1]$. It follows immediately from the construction that $\bar{g}_{n}(\cdot)$ is monotonically decreasing and $\underline{g}_{n}(\cdot)$ is monotonically increasing.

Since $\omega \in N^{c}$ is fixed according to equation (5.32) both $\lim _{n \rightarrow \infty} \bar{g}_{n}(\alpha)=\bar{g}(\alpha)$ and $\lim _{n \rightarrow \infty} \underline{g}_{n}(\alpha)=\underline{g}(\alpha)$ holds for every $\alpha \in \Lambda_{0}$.

Choose an arbitrary $\alpha_{0} \in \Lambda$ and let $\epsilon>0$, then by construction there exists $\delta>0$ such that for every $\alpha \in\left(\alpha_{0}-\delta, \alpha_{0}+\delta\right)$ both

$\bar{g}(\alpha) \in\left(\bar{g}\left(\alpha_{0}\right)-\frac{\epsilon}{2}, \bar{g}\left(\alpha_{0}\right)+\frac{\epsilon}{2}\right) \quad$ and $\quad \underline{g}(\alpha) \in\left(\underline{g}\left(\alpha_{0}\right)-\frac{\epsilon}{2}, \underline{g}\left(\alpha_{0}\right)+\frac{\epsilon}{2}\right)$

is satisfied. Choose points $\alpha_{1}, \alpha_{2} \in \Lambda_{0}$ fulfilling $\alpha_{0}-\delta<\alpha_{1}<\alpha_{0}<\alpha_{2}<$ $\alpha_{0}+\delta$, then there exists an integer $n_{0} \in \mathbb{N}$, such that

$$
\max _{i \in\{1,2\}}\left\{\max \left\{\left|\bar{g}_{n}\left(\alpha_{i}\right)-\bar{g}\left(\alpha_{i}\right)\right|,\left|\underline{g}_{n}\left(\alpha_{i}\right)-\underline{g}\left(\alpha_{i}\right)\right|\right\}\right\}<\frac{\epsilon}{2} \quad \text { for all } n \geq n_{0} .
$$

Using monotonicity this implies that both

$$
\left|\bar{g}_{n}\left(\alpha_{0}\right)-\bar{g}\left(\alpha_{0}\right)\right|<\epsilon \quad \text { and } \quad\left|\underline{g}_{n}\left(\alpha_{0}\right)-\underline{g}\left(\alpha_{0}\right)\right|<\epsilon
$$

holds for every $n \geq n_{0}$, which directly shows that $\lim _{n \rightarrow \infty} \bar{g}_{n}\left(\alpha_{0}\right)=\bar{g}\left(\alpha_{0}\right)$ and $\lim _{n \rightarrow \infty} \underline{g}_{n}\left(\alpha_{0}\right)=\underline{g}\left(\alpha_{0}\right)$ since $\epsilon>0$ was arbitrary. This completes the proof of the theorem.

Having this a Strong Law of Large Numbers (SLLN) for fuzzy relative frequencies and fuzzy probability distributions induced by fuzzy random vectors can be stated and proved as follows $\left(\delta_{H, p}^{\star}\right.$ defined as in Section 1.5 and $\mathbb{P}^{\star}(\cdot)$ defined as in Section 4.3):

\section{Theorem 5.24 (SLLN for fuzzy relative frequencies)}

Suppose that $(\Omega, \mathcal{A}, \mathcal{P})$ is a complete probability space, that $X^{\star}, X_{1}^{\star}, X_{2}^{\star}, \cdots$ are pairwise independent, identically distributed d-dimensional fuzzy random vectors, and that $B \in \mathcal{B}\left(\mathbb{R}^{d}\right)$ is an arbitrary Borel set.

Then there exists a set $N \in \mathcal{A}$, fulfilling $\mathcal{P}(N)=0$, such that for every $\omega \in N^{c}$ the following identity holds $(p \in[1, \infty)$ arbitrary):

$$
\lim _{n \rightarrow \infty} \delta_{H, p}^{\star}\left(h_{n}^{\star}(B, \omega), \mathbb{P}^{\star}(B)\right)=0
$$


Proof: Again suppose that $B \in \mathcal{B}\left(\mathbb{R}^{d}\right)$ is fixed. Since for every $\omega \in \Omega$ by defnition $h_{n}^{\star}(B, \omega)$ is the convex hull of the family $\left(\left[\underline{h}_{n, \alpha}(B, \omega), \bar{h}_{n, \alpha}(B, \omega)\right]\right)_{\alpha \in(0,1]}$, according to (2.5) for every $n \in \mathbb{N}$

$$
\left[h_{n}^{\star}(B, \omega)\right]_{\alpha}=\left[\underline{h}_{n, \alpha}(B, \omega), \bar{h}_{n, \alpha}(B, \omega)\right]
$$

holds for all except (at most) finitely many $\alpha \in(0,1]$. Since a countable union of finite sets is countable, it follows immediately that for all except countably many $\alpha \in(0,1](5.34)$ even is satisfied simultaneously for all $n \in \mathbb{N}$. Consequently applying Theorem 5.23 shows the existence of set $\Lambda^{\prime} \subseteq \Lambda \subseteq(0,1$, fulfilling $\lambda\left(\Lambda^{\prime}\right)=1$, and of a set $N \in \mathcal{A}$, fulfilling $\mathcal{P}(N)=0$, such that for every $\omega \in N^{c}$ and every $\alpha \in \Lambda^{\prime}$

$$
\lim _{n \rightarrow \infty} \delta_{H}\left(\left[h_{n}^{\star}(B, \omega)\right]_{\alpha},\left[\mathbb{P}^{\star}(B)\right]_{\alpha}\right)=0
$$

holds (note that $\delta_{H}(K, L)=\max \{|d-b|,|c-a|\}$ holds for intervals $K:=[a, b]$ and $L:=[c, d])$.

Consequently for arbitrary $p \geq 1$ using Lemma 1.38 and Lebesgue's Dominated Convergence Theorem immediately yields

$$
\lim _{n \rightarrow \infty} \delta_{H, p}^{\star}\left(h_{n}^{\star}(B, \omega), \mathbb{P}^{\star}(B)\right)=0,
$$

for $\omega \in N^{c}$, which had to be proved.

Proposition 5.25 Under the assumptions of Theorem 5.24 the convergence (5.33) also holds with $\delta_{p}^{\star}(\cdot, \cdot)$ replaced by the metric $\rho_{p}^{\star}(\cdot, \cdot)$ (defined as in Section 4.3) for any $p \geq 1$.

Proof: This is an immediate consequence of Theorem 1.46 and Theorem 5.24 .

Remark: Theorem 5.24 is a strong generalization of the SLLN stated in [32]. 


\section{Chapter 6}

\section{Appendix}

\subsection{Short introduction to Souslin sets}

Much of the material presented in this section can be found in [38].

Throughout this section $\mathbb{N}^{\mathbb{N}}$ denotes the set of all sequences in $\mathbb{N}=\{1,2, \ldots\}$, $\mathbb{N}<\mathbb{N}$ denotes the set of all finite sequences in $\mathbb{N}$ including the empty sequence, which will be denoted by $e$. Elements of $\mathbb{N}^{\mathbb{N}}$ will be written in the form $\sigma=\left(\sigma_{1}, \sigma_{2}, \ldots\right)$ and elements of $\mathbb{N}<\mathbb{N}$ in the form $\left(s_{1}, \ldots, s_{n}\right)$.

For $\sigma \in \mathbb{N}^{\mathbb{N}}$ and $k \in \mathbb{N}$ the finite sequence $\sigma \mid k:=\left(\sigma_{1}, \ldots, \sigma_{k}\right)$ is called the initial segment of $\sigma$ of length $k$. If $s \in \mathbb{N}<\mathbb{N}$ then $|s|$ denotes the length of $s$. Furthermore if $s=\left(s_{1}, \ldots, s_{n}\right), t=\left(t_{1}, \ldots, t_{m}\right) \in \mathbb{N}<\mathbb{N}$, then $t$ is called initial segment of $s$ and $s$ is called (possible) extension of $t$ if $m<n$ and $s_{i}=t_{i} \forall i \in\{1, \ldots, m\}$. If $t$ is an initial segment of $s$ this will briefly be expressed by $t \prec s$.

Definition 6.1 Let $\Omega$ be an arbitrary set and denote by $\mathfrak{F}$ a family of subsets of $\Omega$. Then a function $\Phi: \mathbb{N}<\mathbb{N} \longrightarrow \mathfrak{F}, s \mapsto \Phi(s)=: A_{s}$ is called a tree in $\mathfrak{F}$. (The family $\left\{A_{s}: s \in \mathbb{N}<\mathbb{N}\right\}$ will be called tree in $\mathfrak{F}$ as well).

Furthermore a tree $\Phi$ is called regular, if $A_{t} \supseteq A_{s}$ whenever $t \prec s$ holds.

Remark: If $\left\{A_{s}: s \in \mathbb{N}^{<\mathbb{N}}\right\}$ is a tree in $\mathfrak{F}$ then it is possible to derive a regular tree $\left\{B_{s}: s \in \mathbb{N}^{<\mathbb{N}}\right\}$ by simply defining

$$
B_{\left(s_{1}, \ldots, s_{n}\right)}:=\bigcap_{k=1}^{n} A_{\left(s_{1}, \ldots, s_{k}\right)}
$$

for every $s=\left(s_{1}, \ldots, s_{n}\right) \in \mathbb{N}<\mathbb{N}$. 
Definition 6.2 Let $\Omega$ be an arbitrary set, $\mathfrak{F}$ a family of subsets of $\Omega$ and $\left\{A_{s}: s \in \mathbb{N}^{<\mathbb{N}}\right\}$ a tree in $\mathfrak{F}$, then the set $\mathcal{G}\left(\left\{A_{s}\right\}\right)$, defined by

$$
\mathcal{G}\left(\left\{A_{s}\right\}\right):=\bigcup_{\sigma \in \mathbb{N}^{\mathbb{N}}} \bigcap_{n=1}^{\infty} A_{\left(\sigma_{1}, \ldots, \sigma_{n}\right)}=\bigcup_{\sigma \in \mathbb{N}^{\mathbb{N}}} \bigcap_{n=1}^{\infty} A_{\sigma \mid n},
$$

is called Souslin set generated by the tree $\left\{A_{s}: s \in \mathbb{N}<\mathbb{N}\right\}$.

Furthermore the family of all Souslin sets generated by $\mathfrak{F}$ will be denoted by $\mathcal{G}_{\text {sous }}(\mathfrak{F})$, i.e.

$$
\mathcal{G}_{\text {sous }}(\mathfrak{F})=\left\{\mathcal{G}\left(\left\{A_{s}\right\}\right):\left\{A_{s}: s \in \mathbb{N}^{<\mathbb{N}}\right\} \text { is a tree in } \mathfrak{F}\right\} .
$$

The following lemma shows that the Souslin operation contains countable unions and countable intersections.

Lemma 6.3 Let $\Omega$ be an arbitrary set and $\mathfrak{F}$ a family of subsets of $\Omega$, then $\mathcal{G}_{\text {sous }}(\mathfrak{F})$ contains $\mathfrak{F}$ and all countable unions and countable intersections of elements in $\mathfrak{F}$. In other words: $\mathfrak{F}, \mathfrak{F}_{\sigma}, \mathfrak{F}_{\delta} \subseteq \mathcal{G}_{\text {sous }}(\mathfrak{F})$

Proof: Suppose that $A \in \mathfrak{F}$ and set $\Phi(s)=A_{s}=A$ for every $s \in \mathbb{N}<\mathbb{N}$, which gives $\mathcal{G}\left(\left\{A_{s}\right\}\right)=A \in \mathcal{G}_{\text {sous }}(\mathfrak{F})$. This proves that $\mathfrak{F} \subseteq \mathcal{G}_{\text {sous }}(\mathfrak{F})$.

Next let $\left(B_{n}\right)_{n \in \mathbb{N}}$ be a sequence in $\mathfrak{F}$ and set $A_{s}=B_{i}$ for every $s \in \mathbb{N}<\mathbb{N}$ with $s=\left(i, s_{2}, s_{3}, \ldots\right)$. This gives

$$
\mathcal{G}\left(\left\{A_{s}\right\}\right)=\bigcup_{i=1}^{\infty} B_{i} \in \mathcal{G}_{\text {sous }}(\mathfrak{F})
$$

and shows that $\mathfrak{F}_{\sigma} \subseteq \mathcal{G}_{\text {sous }}(\mathfrak{F})$. Furthermore if $\left(B_{n}\right)_{n \in \mathbb{N}}$ is a sequence in $\mathfrak{F}$, then setting $A_{s}=B_{i}$ if $|s|=i$ gives a tree $\left\{A_{s}: s \in \mathbb{N}^{<\mathbb{N}}\right\}$ fulfilling

$$
\mathcal{G}\left(\left\{A_{s}\right\}\right)=\bigcap_{i=1}^{\infty} B_{i} \in \mathcal{G}_{\text {sous }}(\mathfrak{F})
$$

This shows that $\mathfrak{F}_{\delta} \subseteq \mathcal{G}_{\text {sous }}(\mathfrak{F})$ and completes the proof of the lemma.

The following Theorem on the idempotence of the Souslin operation can be considered as one of the most important results in the theory of Souslin sets and plays an important part throughout this section.

Theorem 6.4 Suppose $\mathfrak{F}$ is a family of subsets of a given set $\Omega$, then

$$
\mathcal{G}_{\text {sous }}\left(\mathcal{G}_{\text {sous }}(\mathfrak{F})\right)=\mathcal{G}_{\text {sous }}(\mathfrak{F})
$$


Proof: Because of Lemma $6.3 \mathcal{G}_{\text {sous }}\left(\mathcal{G}_{\text {sous }}(\mathfrak{F})\right) \supseteq \mathcal{G}_{\text {sous }}(\mathfrak{F})$ holds. Therefore it suffices to prove the other inclusion.

If $A \in \mathcal{G}_{\text {sous }}\left(\mathcal{G}_{\text {sous }}(\mathfrak{F})\right)$ then there exists a tree $\left\{A_{s}: s \in \mathbb{N}^{<\mathbb{N}}\right\}$ in $\mathcal{G}_{\text {sous }}(\mathfrak{F})$ such that

$$
A=\mathcal{G}\left(\left\{A_{s}: s \in \mathbb{N}^{<\mathbb{N}}\right\}\right)=\bigcup_{\alpha \in \mathbb{N}^{\mathbb{N}}} \bigcap_{n=1}^{\infty} A_{\alpha \mid n} .
$$

For every $s \in \mathbb{N}^{<\mathbb{N}}$ and $A_{s}$ there exists a tree $\left\{B_{t}^{s}: t \in \mathbb{N}^{<\mathbb{N}}\right\}$ in $\mathfrak{F}$ fulfilling

$$
A_{s}=\mathcal{G}\left(\left\{B_{t}^{s}: t \in \mathbb{N}^{<\mathbb{N}}\right\}\right)=\bigcup_{\gamma \in \mathbb{N}^{\mathbb{N}}} \bigcap_{m=1}^{\infty} B_{\gamma \mid m}^{s} .
$$

Consequently

$$
A=\bigcup_{\alpha \in \mathbb{N}^{\mathbb{N}}} \bigcap_{n=1}^{\infty} \bigcup_{\gamma \in \mathbb{N}^{\mathbb{N}}} \bigcap_{m=1}^{\infty} B_{\gamma \mid m}^{\alpha \mid n}
$$

In other words:

$$
\begin{aligned}
x \in A & \Longleftrightarrow \exists \alpha \in \mathbb{N}^{\mathbb{N}}: \text { for every } n \in \mathbb{N}: \exists \gamma_{n} \in \mathbb{N}^{\mathbb{N}}: x \in B_{\gamma_{n} \mid m}^{\alpha \mid n} \forall m \in \mathbb{N} \\
& \Longleftrightarrow \exists \alpha \in \mathbb{N}^{\mathbb{N}}: \exists\left(\gamma_{n}\right)_{n \in \mathbb{N}} \in\left(\mathbb{N}^{\mathbb{N}}\right)^{\mathbb{N}}: x \in B_{\gamma_{n} \mid m}^{\alpha \mid n} \forall m, n \in \mathbb{N}
\end{aligned}
$$

It suffices to prove that there exists a tree $\left\{C_{s}: s \in \mathbb{N}<\mathbb{N}\right\}$ in $\mathfrak{F}$ such that $A=\mathcal{G}\left(\left\{C_{s}\right\}\right)$.

Claim: There exist bijections $u: \mathbb{N} \times \mathbb{N} \longrightarrow \mathbb{N}, v: \mathbb{N}^{\mathbb{N}} \times\left(\mathbb{N}^{\mathbb{N}}\right)^{\mathbb{N}} \longrightarrow \mathbb{N}^{\mathbb{N}}$ and mappings $\varphi, \psi: \mathbb{N}<\mathbb{N} \longrightarrow \mathbb{N}^{<\mathbb{N}}$ such that for every $\left(\alpha,\left(\gamma_{i}\right)\right) \in: \mathbb{N}^{\mathbb{N}} \times\left(\mathbb{N}^{<N}\right)^{\mathbb{N}}$ the following holds:

If $v\left(\alpha,\left(\gamma_{i}\right)\right)=\beta$ and $s=\beta \mid u(n, m)$ for some $n, m \in \mathbb{N}$ then $\varphi(s)=\alpha \mid n$ and $\psi(s)=\gamma_{n} \mid m$.

For the moment assume that such functions exist and define

$$
C_{s}=B_{\psi(s)}^{\varphi(s)} \in \mathfrak{F} \quad \text { for every } s \in \mathbb{N}<\mathbb{N} .
$$

If $x \in A$ it follows by the above series of equivalences that there exists $\alpha \in \mathbb{N}^{\mathbb{N}}$ and $\left(\gamma_{i}\right) \in\left(\mathbb{N}^{\mathbb{N}}\right)^{\mathbb{N}}$ such that $x \in B_{\gamma_{n} \mid m}^{\alpha \mid n}$ for all $m, n \in \mathbb{N}$.

Set $\beta:=v\left(\alpha,\left(\gamma_{i}\right)\right)$ and take any $k \in \mathbb{N}$, then there exist unique $n, m \in \mathbb{N}$ with $u(n, m)=k$. Therefore $\varphi(\beta \mid k)=\alpha\left|n, \psi(\beta \mid k)=\gamma_{n}\right| m$ and $x \in C_{\beta \mid k}=B_{\gamma_{n} \mid m}^{\alpha \mid n}$. Since $k$ was arbitrary it follows that

$$
x \in \bigcap_{k=1}^{\infty} C_{\beta \mid k} \subseteq \bigcup_{\sigma \in \mathbb{N}^{\mathbb{N}}} \bigcap_{k=1}^{\infty} C_{\sigma \mid k}=\mathcal{G}\left(\left\{C_{s}\right\}\right) .
$$


This proves that $A \subseteq \mathcal{G}\left(\left\{C_{s}\right\}\right)$.

On the other hand if $x \in \mathcal{G}\left(\left\{C_{s}\right\}\right)$ then there exists $\beta \in \mathbb{N}^{\mathbb{N}}$ such that $x \in C_{\beta \mid k}$ for every $k \in \mathbb{N}$. Choose $\left(\alpha,\left(\gamma_{i}\right)\right) \in \mathbb{N}^{\mathbb{N}} \times\left(\mathbb{N}^{\mathbb{N}}\right)^{\mathbb{N}}$ such that $v\left(\alpha,\left(\gamma_{i}\right)\right)=\beta$.

Fix $m, n \in \mathbb{N}$ and set $k=u(n, m)$ then it follows again that $\varphi(\beta \mid k)=\alpha \mid n$ and $\psi(\beta \mid k)=\gamma_{n} \mid m$. Consequently $x \in B_{\gamma_{n} \mid m}^{\alpha \mid n}$. Since $m, n \in \mathbb{N}$ were chosen arbitrarily it follows immediately that $x \in A$.

This shows that $\mathcal{G}\left(\left\{C_{s}\right\}\right) \subseteq A$ and completes the proof if the claim is proved.

Proof of the Claim: In order to keep notation as concise as possible, the following will be used throughout the proof:

If $\sigma \in \mathbb{N}^{\mathbb{N}}$ then $\sigma(k):=\sigma_{k}$ denotes the $k$-th coordinate for every $k \in \mathbb{N}$.

(i) Define a function $u: \mathbb{N} \times \mathbb{N} \longrightarrow \mathbb{N}$ by

$$
u(n, m)=2^{n-1}(2 m-1), \quad n, m \in \mathbb{N} .
$$

Since $u\left(n^{\prime}, m^{\prime}\right)=u(n, m)$ for $n, m, n^{\prime}, m^{\prime} \in \mathbb{N}$ is equivalent to

$$
2 m^{\prime}-1=2^{n-n^{\prime}}(2 m-1)
$$

it follows immediately that $n=n^{\prime}$ and therefore $m=m^{\prime}$, which proves that $u$ is one-to-one. Furthermore $u$ is easily seen to be onto, since every natural number can be decomposed in a product of primes.

Obviously $u$ is (strictly) monotonically increasing in both coordinates and fulfills $n \leq u(n, m)$ for all $n, m \in \mathbb{N}$.

Let the function $(l, r): \mathbb{N} \longrightarrow \mathbb{N} \times \mathbb{N}$ be defined in the following way

$$
(l(k), r(k))=(i, j) \quad \Longleftrightarrow u(i, j)=k \quad \text { for all } i, j, k \in \mathbb{N} .
$$

(In other words $(l, r)$ is the inverse function of $u$.)

(ii) Given $u$ define a function $v: \mathbb{N}^{\mathbb{N}} \times\left(\mathbb{N}^{\mathbb{N}}\right)^{\mathbb{N}} \longrightarrow \mathbb{N}^{\mathbb{N}}$ in the following way:

$$
v\left(\alpha,\left(\gamma_{i}\right)\right)(k)=u\left(\alpha(k), \gamma_{l(k)}(r(k))\right) \quad \text { for } k \in \mathbb{N}
$$

Suppose that $\left(\alpha,\left(\gamma_{i}\right)\right),\left(\alpha^{\prime},\left(\gamma_{i}^{\prime}\right)\right) \in \mathbb{N}^{\mathbb{N}} \times\left(\mathbb{N}^{\mathbb{N}}\right)^{\mathbb{N}}$ and $\left(\alpha,\left(\gamma_{i}\right)\right) \neq\left(\alpha^{\prime},\left(\gamma_{i}^{\prime}\right)\right)$.

If $\alpha \neq \alpha^{\prime}$ then there exists $k \in \mathbb{N}$ such that $\alpha(k) \neq \alpha^{\prime}(k)$. Since $u$ is one-to-one it follows that

$$
u\left(\alpha(k), \gamma_{l(k)}(r(k))\right) \neq u\left(\alpha^{\prime}(k), \gamma_{l(k)}^{\prime}(r(k))\right)
$$

and therefore

$$
v\left(\alpha,\left(\gamma_{i}\right)\right) \neq v\left(\alpha^{\prime},\left(\gamma_{i}^{\prime}\right)\right)
$$


If, on the other hand $\gamma_{i} \neq \gamma_{i}^{\prime}$ for some $i \in \mathbb{N}$ then there exists $j \in \mathbb{N}$ such that $\gamma_{i}(j) \neq \gamma_{i}^{\prime}(j)$. Choose $k$ so that $l(k)=i$ and $r(k)=j$, then it follows immediately that

$$
v\left(\alpha,\left(\gamma_{i}\right)\right)(k)=u\left(\alpha(k), \gamma_{l(k)}(r(k))\right) \neq u\left(\alpha^{\prime}(k), \gamma_{l(k)}^{\prime}(r(k))\right)=v\left(\alpha^{\prime},\left(\gamma_{i}^{\prime}\right)\right)(k)
$$

Therefore $v$ is one-to-one.

In order to show that $v$ is also onto, let $\beta \in \mathbb{N}^{\mathbb{N}}$ be arbitrary and define $\left(\alpha,\left(\gamma_{i}\right)\right) \in \mathbb{N}^{\mathbb{N}} \times\left(\mathbb{N}^{\mathbb{N}}\right)^{\mathbb{N}}$ by

$$
\alpha(k)=l(\beta(k)), \quad \gamma_{i}(j)=r(\beta(u(i, j))) \quad \text { for all } i, j, k \in \mathbb{N} .
$$

It follows immediately that

$$
v\left(\left(\alpha,\left(\gamma_{i}\right)\right)\right)(k)=u\left(\alpha(k), \gamma_{l(k)}(r(k))\right)=u(l(\beta(k)), r(\beta(k)))=\beta(k),
$$

which proves that $v$ is onto, and therefore bijective.

(iii) It remains to construct the functions $\varphi, \psi$.

Let $s \in \mathbb{N}^{<\mathbb{N}}$ arbitrary. Pick $\beta \in \mathbb{N}^{\mathbb{N}}$ and $k \in \mathbb{N}$ so that $s=\beta \mid k$ holds. Furthermore choose $\left(\alpha,\left(\gamma_{i}\right)\right) \in \mathbb{N}^{\mathbb{N}} \times\left(\mathbb{N}^{\mathbb{N}}\right)^{\mathbb{N}}$ and $n, m \in \mathbb{N}$ fulfilling $v\left(\left(\alpha,\left(\gamma_{i}\right)\right)\right)=\beta$ and $u(n, m)=k$. Having this define the function $\varphi: \mathbb{N}<\mathbb{N} \longrightarrow \mathbb{N}<\mathbb{N}$ by

$$
\varphi(s)=(l(\beta(1)), \ldots, l(\beta(n))) .
$$

Since $n \leq u(n, m)=k$ the definition makes sense. Using (6.5) it follows immediately that

$$
\varphi(s)=\varphi(\beta \mid k)=(l(\beta(1)), \ldots, l(\beta(n)))=(\alpha(1), \ldots, \alpha(n))=\alpha \mid n .
$$

In the same manner define the function $\psi: \mathbb{N}<\mathbb{N} \longrightarrow \mathbb{N}^{<N}$ by

$$
\varphi(s)=(r(\beta(u(n, 1))), \ldots, r(\beta(u(n, m)))) .
$$

Since $u(n, m)=k$ it follows that $u(n, i) \leq k$ for every $i \in\{1, \ldots, m\}$, which shows that $\psi$ is well defined. Again using (6.5) gives

$$
\begin{aligned}
\psi(s) & =\psi(\beta \mid k)=(r(\beta(u(n, 1))), \ldots, r(\beta(u(n, m)))) \\
& =\left(\gamma_{n}(1), \ldots, \gamma_{n}(m)\right)=\gamma_{n} \mid m .
\end{aligned}
$$

As $s$ was arbitrary this completes both the proof of the Claim and the proof of Theorem 6.4. 
Theorem 6.5 Suppose $\mathfrak{F}$ is a family of subsets of a given set $\Omega$, then $\mathcal{G}_{\text {sous }}(\mathfrak{F})$ is closed under countable unions and countable intersections.

Proof: According to Lemma $6.3 \mathfrak{F}_{\sigma}, \mathfrak{F}_{\delta} \subseteq \mathcal{G}_{\text {sous }}(\mathfrak{F})$ holds for every family $\mathfrak{F}$ of subsets of $\Omega$. Therefore

$$
\begin{aligned}
& \left(\mathcal{G}_{\text {sous }}(\mathfrak{F})\right)_{\delta} \subseteq \mathcal{G}_{\text {sous }}\left(\mathcal{G}_{\text {sous }}(\mathfrak{F})\right)=\mathcal{G}_{\text {sous }}(\mathfrak{F}) \quad \text { and } \\
& \left(\mathcal{G}_{\text {sous }}(\mathfrak{F})\right)_{\sigma} \subseteq \mathcal{G}_{\text {sous }}\left(\mathcal{G}_{\text {sous }}(\mathfrak{F})\right)=\mathcal{G}_{\text {sous }}(\mathfrak{F})
\end{aligned}
$$

This completes the proof.

So far, no assumption, like being closed under unions or intersection, were imposed on the family $\mathfrak{F}$. Especially if $\mathfrak{F}$ is a $\sigma$-algebra $\mathcal{A}$ the question naturally arises whether there exists a Souslin set $A \in \mathcal{G}_{\text {sous }}(\mathcal{A})$ that is not measurable, i.e. $A \notin \mathcal{A}$. It will be proved that the answer is no if $(\Omega, \mathcal{A}, \mu)$ is a complete $\sigma$-finite measure space.

Before doing that a first result is presented in order to demonstrate how large the family of Souslin sets is, if the generator $\mathfrak{F}$ is the family $\mathcal{O}$ of all open sets in a metric space $(\Omega, d)$ :

Theorem 6.6 Suppose that $(\Omega, d)$ is an arbitrary metric space. Denote by $\mathcal{O}$ the topology induced by the metric $d$ and by $\mathcal{B}(\Omega)$ the Borel $\sigma$-algebra (generated by the topology). Then it follows that $\mathcal{B}(\Omega) \subseteq \mathcal{G}_{\text {sous }}(\mathcal{O})$.

Proof: It is well known that every closed subset of a metric space is a countable intersection of open sets. Namely if $F$ is closed then obviously

$$
F=\bigcap_{n=1}^{\infty} K\left(F, \frac{1}{n}\right),
$$

where $K(F, \epsilon)=\{x \in \Omega: \exists y \in F$ with $d(x, y)<\epsilon\} \in \mathcal{O}$ for every $\epsilon>0$.

Claim: $\mathcal{B}(\Omega)$ is the smallest collection in $2^{\Omega}$ containing $\mathcal{O}$, that is closed under countable intersections and countable unions.

Suppose that $\mathcal{R} \subseteq \mathcal{B}(\Omega)$ is closed under countable intersections and countable unions and that $\mathcal{R}$ contains $\mathcal{O}$. Defining $\mathcal{R}^{\prime}:=\left\{A \in \mathcal{R}: A^{c} \in \mathcal{R}\right\} \subseteq \mathcal{R}$ it follows that $\mathcal{R}^{\prime}$ is closed under complementation and countable unions. Therefore $\mathcal{R}^{\prime}$ is a $\sigma$-algebra. Using the fact that for every open set $B$ the complement $B^{c}$ is a countable intersection of open sets it follows that $B^{c} \in \mathcal{R}$, proving that $\mathcal{O} \subseteq \mathcal{R}^{\prime}$. Applying

$$
\mathcal{O} \subseteq \mathcal{R}^{\prime} \subseteq \mathcal{R} \subseteq \mathcal{B}(\Omega) \subseteq \mathcal{R}^{\prime}
$$

gives $\mathcal{R}^{\prime}=\mathcal{R}=\mathcal{B}(\Omega)$, which completes the proof of the Claim.

Because of $\mathcal{O} \subseteq \mathcal{G}_{\text {sous }}(\mathcal{O})$ and the fact that according to Theorem 6.5 $\mathcal{G}_{\text {sous }}(\mathcal{O})$ 
is closed under countable intersections and countable unions it finally follows that $\mathcal{B}(\Omega) \subseteq \mathcal{G}_{\text {sous }}(\mathcal{O})$.

As preintimated the case where $\mathfrak{F}$ is a $\sigma$-algebra will now be analyzed. Remember the following definition.

Definition 6.7 A measure space $(\Omega, \mathcal{A}, \mu)$ is called complete if every subset of a set $A \in \mathcal{A}$ of measure zero belongs to $\mathcal{A}$.

Remark: It is well known that every measure space $(\Omega, \mathcal{A}, \mu)$ can be completed easily. One just has to consider the outer measure $\mu^{*}: 2^{\Omega} \rightarrow \mathbb{R}$ induced by $\mu$, which is defined by

$$
\mu^{*}(A)=\inf \{\mu(B): A \subseteq B \text { and } B \in \mathcal{A}\},
$$

and the $\sigma$-algebra $\mathcal{A}^{*}$ of all $\mu^{*}$-mesurable sets. This gives a complete mesure space $\left(\Omega, \mathcal{A}^{*}, \mu^{*}\right)$ fulfilling $\mathcal{A} \subseteq \mathcal{A}^{*}$ (compare for instance [2], [13]).

Another also well known method, which provides a complete extension $\bar{\mu}$ of $\mu$ with minimal domain, called completion of $\mu$, works as follows:

Suppose that $(\Omega, \mathcal{A}, \mu)$ is a non-complete measure space and denote with $\mathcal{N}$ the family of all subsets of sets of measure zero. Futhermore define

$$
\begin{aligned}
\bar{A} & :=\{A \cup N: A \in \mathcal{A} \text { and } N \in \mathcal{N}\} \quad \text { and } \\
\bar{\mu}(A \cup N) & :=\mu(A) \quad \text { for } A \in \mathcal{A}, N \in \mathcal{N} .
\end{aligned}
$$

It can be shown easily that $\overline{\mathcal{A}}$ is a $\sigma$-algebra and that $(\Omega, \overline{\mathcal{A}}, \bar{\mu})$ is a complete measure space. Moreover if $\mu$ is $\sigma$-finite, it can be proved that both constructions yield the same result, i.e. $\overline{\mathcal{A}}=\mathcal{A}^{*}$ (compare [13]).

The following lemmas will be very helpful.

Lemma 6.8 Suppose that $(\Omega, \mathcal{A}, \mu)$ is an arbitrary measure space and denote by $\mu^{*}$ the outer measure defined according to (6.6). Then for every $A \subseteq \Omega$ there exists a set $\hat{A} \in \mathcal{A}$ such that $A \subseteq \hat{A}$ and $\mu(\hat{A})=\mu^{*}(A)$.

Proof: Let $A \subseteq \Omega$ be arbitrary. If $\mu^{*}(A)=\infty$ then set $A=\Omega$.

If $\mu^{*}(A)<\infty$ it follows by (6.6) that for every $n \in \mathbb{N}$ there exists a set $A_{n} \in \mathcal{A}$ fulfilling $A \subseteq A_{n}$ and $\mu\left(A_{n}\right) \leq \mu^{*}(A)+\frac{1}{n}$. Set $B_{n}=\bigcap_{k=1}^{n} A_{k}$ for every $n \in \mathbb{N}$ and $\hat{A}=\bigcap_{n=1}^{\infty} B_{n}$, then it follows immediately that $A \subseteq \hat{A}$, $B_{n} \searrow \hat{A}$ and $\hat{A} \in \mathcal{A}$. Consequently

$$
\mu^{*}(A) \leq \mu^{*}\left(B_{n}\right)=\mu\left(B_{n}\right) \leq \mu\left(A_{n}\right) \leq \mu^{*}(A)+\frac{1}{n} \quad \text { for every } n \in \mathbb{N},
$$

from which $\mu(\hat{A})=\mu^{*}(A)$ immediately follows by considering $n \rightarrow \infty$. This completes the proof of the lemma. 
Lemma 6.9 Suppose that $(\Omega, \mathcal{A}, \mu)$ is a $\sigma$-finite measure space. Then for every $A \subseteq \Omega$ there exists a set $\hat{A} \in \mathcal{A}$ such that $A \subseteq \hat{A}$ and $\mu(\hat{A} \backslash B)=0$ for every set $B \in \mathcal{A}$ containing $A$.

If in addition $(\Omega, \mathcal{A}, \mu)$ is complete, then every subset of $\hat{A} \backslash B$ is measurable.

Proof: Again let $\mu^{*}$ denote the outer measure induced by $\mu$ according to (6.6) and suppose that $A \subseteq \Omega$. If $\mu^{*}(A)<\infty$ choose $\hat{A}$ according to Lemma 6.8 and suppose that Lemma 6.9 does not hold. Then there exists a set $B \in \mathcal{A}$ with $A \subseteq B$ and $\mu(\hat{A} \backslash B)>0$. Defining $\tilde{A}:=\hat{A} \backslash(\hat{A} \backslash B)$ it follows immediately that $\tilde{A} \cap(\hat{A} \backslash B)=\emptyset, \tilde{A} \cup(\hat{A} \backslash B)=\hat{A}$ and $A \subseteq \tilde{A}$. Therefore

$$
\mu^{*}(A) \leq \mu^{*}(\tilde{A})=\mu(\tilde{A})=\mu(\hat{A})-\mu(\hat{A} \backslash B)=\mu^{*}(A)-\mu(\hat{A} \backslash B)<\mu^{*}(A),
$$

which is a contradiction and proves that $\mu(\hat{A} \backslash B)=0$ for every set $B \in \mathcal{A}$ containing $A$.

If on the other hand $\mu^{*}(A)=\infty$, then choose a countable partition $\left(C_{i}\right)_{i \in \mathbb{N}}$ of $\Omega$ such that $\mu\left(C_{i}\right)<\infty$ for every $i \in \mathbb{N}$, which is possible since $(\Omega, \mathcal{A}, \mu)$ is $\sigma$-finite by assumption. Define $A_{i}:=A \cap C_{i}$ for every $i \in \mathbb{N}$, choose $\hat{A}_{i}$ according to Lemma 6.8 and set $\hat{A}:=\bigcup_{i=1}^{\infty} \hat{A}_{i} \in \mathcal{A}$.

If $B \in \mathcal{A}$ such that $A \subseteq B$, setting $B_{i}=B \cap C_{i} \in \mathcal{A}$ it follows that

$$
\hat{A} \backslash B=\left(\bigcup_{i=1}^{\infty} \hat{A}_{i}\right) \backslash\left(\bigcup_{k=1}^{\infty} B_{k}\right)=\bigcup_{i=1}^{\infty}\left(\hat{A}_{i} \backslash \bigcup_{k=1}^{\infty} B_{k}\right) \subseteq \bigcup_{i=1}^{\infty}\left(\hat{A}_{i} \backslash B_{i}\right) .
$$

Therefore

$$
\mu(\hat{A} \backslash B) \leq \sum_{i=1}^{\infty} \mu\left(\hat{A}_{i} \backslash B_{i}\right)=0 .
$$

If in addition $(\Omega, \mathcal{A}, \mu)$ is complete, then every subset of $\hat{A} \backslash B$ is measurable since $\mu(\hat{A} \backslash B)=0$. This completes the proof.

Theorem 6.10 If $(\Omega, \mathcal{A}, \mu)$ is a complete $\sigma$-finite measure space, then it follows that $\mathcal{G}_{\text {sous }}(\mathcal{A})=\mathcal{A}$. In other words, in case of a complete $\sigma$-finite measure space, $\mathcal{A}$ is closed under the Souslin operation.

Proof: Suppose that $B \in \mathcal{G}_{\text {sous }}(\mathcal{A})$ is an arbitrary Souslin set generated by a tree $\left\{A_{s}: s \in \mathbb{N}^{<\mathbb{N}}\right\}$ in $\mathcal{A}$. Without loss of generality suppose that $A_{e}=\Omega$ and that the tree $\left\{A_{s}: s \in \mathbb{N}^{<\mathbb{N}}\right\}$ is regular. For every $s \in \mathbb{N}^{<\mathbb{N}}$ define the set $B_{s}$ by

$$
B_{s}:=\bigcup_{\left\{\alpha \in \mathbb{N}^{\mathbb{N}}: s \prec \alpha\right\}} \bigcap_{n=1}^{\infty} A_{\alpha \mid n} .
$$

Obviously $\left\{B_{s}: s \in \mathbb{N}^{<\mathbb{N}}\right\}$ is a regular tree in $\mathcal{A}$ too, $B_{e}=B$ and because of the regularity of $\left\{A_{s}: s \in \mathbb{N}^{<\mathbb{N}}\right\}$ it follows that $B_{s} \subseteq A_{s}$ for every $s \in \mathbb{N}^{<\mathbb{N}}$. 
For the rest of the proof let $s \vee t$ denote the concatenation of $s=\left(s_{1}, \ldots, s_{k}\right)$, $t=\left(t_{1}, \ldots, t_{l}\right) \in \mathbb{N}^{<\mathbb{N}}$, defined by $s \vee t:=\left(s_{1}, \ldots, s_{k}, t_{1}, \ldots, t_{l}\right) \in \mathbb{N}^{<\mathbb{N}}$.

Using this notation it follows that

$$
B_{s}=\bigcup_{n=1}^{\infty} B_{s \vee n} \quad \text { for every } s \in \mathbb{N}^{<\mathbb{N}} \text {. }
$$

For every $s \in \mathbb{N}<\mathbb{N}$ choose $\hat{B}_{s} \in \mathcal{A}$ according to Lemma 6.9 and define

$$
\tilde{B}_{s}:=A_{s} \cap \hat{B}_{s} \quad \text { for every } s \in \mathbb{N}<\mathbb{N} \text {. }
$$

Obviously every set $\tilde{B}_{s}$ inherits the following properties of $\hat{B}_{s}: \mu\left(\tilde{B}_{s} \backslash D\right)=0$ for every set $D \in \mathcal{A}$ containing $B_{s}$ and $E \in \mathcal{A}$ for every set $E \subseteq \tilde{B}_{s} \backslash D$. Furthermore $B_{s} \subseteq \tilde{B}_{s} \subseteq A_{s}$ for every $s \in \mathbb{N}^{<\mathbb{N}}$.

Next define a regular tree $\left\{H_{s}: s \in \mathbb{N}<\mathbb{N}\right\}$ in $\mathcal{A}$ by $H_{e}=\hat{B}$ and

$$
H_{\left(s_{1}, \ldots, s_{n}\right)}:=\bigcap_{k=1}^{n} \tilde{B}_{\left(s_{1}, \ldots, s_{k}\right)}, \quad\left(s_{1}, \ldots, s_{n}\right) \in \mathbb{N}^{<\mathbb{N}} .
$$

Since the tree $\left\{B_{s}: s \in \mathbb{N}^{<\mathbb{N}}\right\}$ is regular it follows that

$$
B_{s} \subseteq H_{s} \subseteq \tilde{B}_{s} \subseteq A_{s} \text { for every } s \in \mathbb{N}^{<\mathbb{N}} .
$$

Consequently $\mu\left(H_{s} \backslash D\right)=0$ for every set $D \in \mathcal{A}$ containing $\dot{B}_{s}$ and $E \in \mathcal{A}$ holds for every set $E \subseteq H_{s} \backslash D$.

Finally define $C_{s}$ for $s \in \mathbb{N}^{<\mathbb{N}}$ by

$$
C_{s}:=H_{s} \backslash \bigcup_{n=1}^{\infty} H_{s \vee n} \in \mathcal{A},
$$

and set

$$
C:=\bigcup_{s \in \mathbb{N}<\mathbb{N}} C_{s}
$$

Using (6.7) and the regularity of $\left\{H_{s}: s \in \mathbb{N}^{<\mathbb{N}}\right\}$ it follows that

$$
B_{s}=\bigcup_{n=1}^{\infty} B_{s \vee n} \subseteq \bigcup_{n=1}^{\infty} H_{s \vee n} \subseteq H_{s} \subseteq \tilde{B}_{s} \subseteq A_{s} \quad \text { for every } s \in \mathbb{N}^{<\mathbb{N}}
$$

Therefore $\mu\left(C_{s}\right)=0$ for every $s \in \mathbb{N}^{<\mathbb{N}}$, from which it follows by using the completeness of $(\Omega, \mathcal{A}, \mu)$, that every subset of $C_{s}$, is measurable. Since $\mathbb{N}<\mathbb{N}$ is as a countable union of countable sets itself countable, it follows that $\mu(C)=0$ and especially that every subset of $C$ is measurable. 
Claim: $H_{e} \backslash B=\hat{B} \backslash B \subseteq C$.

If $x \in \hat{B} \backslash C=H_{e} \backslash C$ it follows that $x \notin C_{e}$, which means by definition that there exists a natural number $\alpha_{1}$ such that $x \in H_{\alpha_{1}} \subseteq A_{\alpha_{1}}$. In the same manner there exists a natural number $\alpha_{2}$ such that $x \in H_{\left(\alpha_{1}, \alpha_{2}\right)} \subseteq A_{\left(\alpha_{1}, \alpha_{2}\right)}$. Proceeding inductively yields a sequence $\alpha=\left(\alpha_{1}, \alpha_{2}, \ldots\right) \in \mathbb{N}^{\mathbb{N}}$ such that

$$
x \in \bigcap_{n=1}^{\infty} A_{\alpha \mid n} \subseteq \bigcup_{\beta \in \mathbb{N}^{N}} \bigcap_{n=1}^{\infty} A_{\beta \mid n}=B
$$

This shows that $\hat{B} \backslash C \subseteq B$. Finally since $B \subseteq \hat{B}$ it follows that $\hat{B} \backslash B \subseteq C$, which completes the proof of the claim.

Now the theorem follows easily: Since $C \in \mathcal{A}$ has measure zero and $\hat{B} \backslash B \subseteq C$ it follows from the completness of the mesure space that $\hat{B} \backslash B \in \mathcal{A}$. Therefore $B=\hat{B} \backslash(\hat{B} \backslash B) \in \mathcal{A}$. This proves that $\mathcal{G}_{\text {sous }}(\mathcal{A}) \subseteq \mathcal{A}$.

The other inclusion is a direct consequence of Lemma 6.3 .

\subsection{Results from functional analysis and mea- sure theory}

The following two theorems can be found in [12],[23],[36]. The first one can be considered as analogon to the theorem of Arzela-Ascoli for $L^{1}(\Omega, \mathcal{A}, \mu)$.

Theorem 6.11 Let $(\Omega, \mathcal{A}, \mu)$ be an arbitrary measure space. Then a family of functions $\mathcal{H} \subseteq L^{1}(\Omega, \mathcal{A}, \mu)$ is weakly precompact if and only if the following three conditions are fulfilled:

1. There exists a constant $M<\infty$ such that $\|f\|_{1} \leq M$ for all $f \in \mathcal{H}$.

2. For every $\epsilon>0$ there exists $\delta>0$ with

$$
\int_{A}|f(x)| d \mu(x)<\epsilon \quad \text { for } \mu(A)<\delta \text { and } f \in \mathcal{H} .
$$

3. For every $\epsilon>0$ there exists a set $B \in \mathcal{A}$ such that $\mu(B)<\infty$ and

$$
\int_{B^{c}}|f(x)| d \mu(x)<\epsilon \quad \text { for all } f \in \mathcal{H} .
$$




\section{Theorem 6.12 (Mazur)}

Let $(E,\|\cdot\|)$ be a normed linear space and $C \subseteq E$ a convex set.

Then the weak closure of $C$ is equal to the strong (norm) closure of $C$.

Especially if $\left(x_{n}\right)_{n \in \mathbb{N}}$ is a sequence in $E$ that converges weakly to some $x \in E$, then there exists a sequence $\left(y_{k}\right)_{k \in \mathbb{N}}$ in $E$ such that

- each $y_{k}$ is a convex combination of finitely many $x_{n}$, and

- $\left\|x-y_{k}\right\| \longrightarrow 0$ for $k \rightarrow \infty$.

The following definition and the following two theorems are taken from [2]:

Definition 6.13 Let $(\Omega, \mathcal{A}, \mu)$ be an arbitrary finite measure space and denote for every $p \geq 1$ by $\mathcal{L}^{p}(\Omega, \mathcal{A}, \mu)$ the set of all measurable real-valued functions $f$, such that $|f|^{p}$ is $\mu$-integrable. Then a family $\mathcal{I} \subseteq \mathcal{L}^{1}(\Omega, \mathcal{A}, \mu)$ is called uniformly integrable if the following two conditions are fulfilled:

1. There exists a constant $M<\infty$ such that $\int_{\Omega}|f(t)| d \mu(t) \leq M$ for all $f \in \mathcal{I}$.

2. For every $\epsilon>0$ there exists $\delta>0$ such that $\int_{A}|f(x)| d \mu(x)<\epsilon$ whenever $\mu(A)<\delta$ and $f \in \mathcal{I}$.

Using uniform integrability it is possible to deduce convergence in $p$-mean as follows:

Theorem 6.14 Let $(\Omega, \mathcal{A}, \mu)$ be a finite measure space and for every $p \geq 1$ denote by $\mathcal{L}^{p}(\Omega, \mathcal{A}, \mu)$ the set of all measurable real-valued functions $f$, such that $|f|^{p}$ is $\mu$-integrable.

If a sequence of functions $\left(f_{n}\right)_{n \in \mathbb{N}}$ in $\mathcal{L}^{p}(\Omega, \mathcal{A}, \mu)$ converges in measure to a measurable function $f$ and if the sequence $\left(\left|f_{n}\right|^{p}\right)_{n \in \mathbb{N}}$ is uniformly integrable, then $f$ is in $\mathcal{L}^{p}(\Omega, \mathcal{A}, \mu)$ and $\left(f_{n}\right)_{n \in \mathbb{N}}$ converges to $f$ in $p$-mean.

On the other hand if a sequence $\left(f_{n}\right)_{n \in \mathbb{N}}$ of functions in $\mathcal{L}^{p}(\Omega, \mathcal{A}, \mu)$ converges in $p$-mean to a measurable function $f$ then the sequence $\left(\left|f_{n}\right|^{p}\right)_{n \in \mathbb{N}}$ is uniformly integrable and $\left(f_{n}\right)_{n \in \mathbb{N}}$ converges in measure to $f$.

Theorem 6.15 (Subsequence principle for convergence in measure) Suppose that $(\Omega, \mathcal{A}, \mu)$ is a finite measure space, then a sequence $\left(f_{n}\right)_{n \in \mathbb{N}}$ of measurable real-valued functions convergens to a measurable real-valued function $f$ in measure if and only if for every subsequence $\left(f_{n_{k}}\right)_{k \in \mathbb{N}}$ of $\left(f_{n}\right)_{n \in \mathbb{N}}$ there exists a further subsequence $\left(f_{n_{k_{j}}}\right)_{j \in \mathbb{N}}$ that converges to $f \mu$-almost everywhere. 


\title{
List of Symbols
}

\author{
$\oplus \ldots \ldots \ldots$. Sum of fuzzy numbers (and fuzzy vectors) \\ $\ominus \ldots \ldots \ldots \ldots$ Difference of fuzzy numbers (and fuzzy vectors) \\ $[\cdot]_{\alpha} \ldots \ldots \ldots \ldots, \alpha$-cut of a fuzzy number or fuzzy vector \\ $|\cdot| \ldots \ldots \ldots . .$. Absolute value (modulus) \\ $\subseteq \ldots \ldots \ldots \ldots$ Inclusion \\ $\preceq \ldots \ldots \ldots \ldots$. Semiordering for fuzzy numbers \\ $\cap \ldots \ldots \ldots$..... Intersection \\ $\cup \ldots \ldots \ldots$ Union \\ $\langle\cdot, \cdot\rangle \ldots \ldots \ldots .$. Standard inner product on $\mathbb{R}^{d}$ \\ o ............ Composition of functions \\ $\|\cdot\|_{p} \ldots \ldots \ldots . L^{p}$-norm \\ $\|\cdot\|_{\infty} \ldots \ldots .$. Uniform norm \\ $\mathbf{1}_{A}(\cdot) \ldots \ldots \ldots$ Indicator function of the set $A$ \\ $2^{\Omega} \ldots \ldots \ldots \ldots$ Set of all subsets of $\Omega$ (power set) \\ $\mathcal{A}_{\sigma}(\cdot) \ldots \ldots \ldots$ Generated $\sigma$-algebra \\ $A+B \ldots \ldots$ Minkowksi sum of the sets $A$ and $B$ \\ $\mu A \ldots \ldots$...... Minkowski scalar multiplication of the set $A$ with the \\ scalar $\mu$ \\ $\bar{A}, \operatorname{cls}(A) \ldots \ldots$ Closure of the set $A$ \\ $A \times B \ldots \ldots$. Cartesian product of $A$ and $B$ \\ $\mathcal{A}_{1} \otimes \mathcal{A}_{2} \ldots \ldots$. Product $\sigma$-algebra of $\mathcal{A}_{1}$ and $\mathcal{A}_{2}$ \\ $B(x, r) \ldots \ldots \ldots$ Open Ball with center $x$ and radius $r$ with respect to the \\ Euclidean norm in $\mathbb{R}^{d}$ \\ $\bar{B}(x, r) \ldots \ldots$ Closed Ball with center $x$ and radius $r$ with respect to the \\ Euclidean norm in $\mathbb{R}^{d}$ \\ $B_{\delta_{H}}(A, r) \ldots \ldots$ Open ball with center $A$ and radius $r$ with respect to the \\ Hausdorff metric $\delta_{H}$ \\ $\mathfrak{B} \ldots \ldots \ldots$ Countable basis of the Euclidean topology on $\mathbb{R}^{d}$ consis- \\ ting of all open balls $B(m, r)$ with $m \in \mathbb{Q}^{d}$ and $r \in \mathbb{Q}^{+}$
}


$\mathcal{B}((0,1]) \ldots \ldots$ Borel $\sigma$-algebra on $(0,1]$

$\mathcal{B}\left(\left(\mathcal{K}^{d}, \delta_{H}\right)\right) \ldots$ Borel $\sigma$-algebra on $\mathcal{K}^{d}$

$\mathcal{B}\left(\left(\mathcal{K}_{c}^{d}, \delta_{H}\right)\right) \ldots$ Borel $\sigma$-algebra on $\mathcal{K}_{c}^{d}$

$\mathcal{B}(\mathbb{R}) \ldots \ldots \ldots$ Borel $\sigma$-algebra on $\mathbb{R}$

$\mathcal{B}\left(\mathbb{R}^{d}\right) \ldots \ldots$ Borel $\sigma$-algebra on $\mathbb{R}^{d}$

$\mathcal{B}\left(S^{d-1}\right) \ldots \ldots$ Borel $\sigma$-algebra on $S^{d-1}$

$C\left(S^{d-1}\right) \ldots \ldots$. Space of all continuous real-valued functions on $S^{d-1}$

$\operatorname{conv}(A) \ldots \ldots$. Convex hull of the set $A$

$\operatorname{diam}(\cdot) \ldots \ldots$. Diameter

d............ Dimension

$\widetilde{\mathcal{D}}_{\alpha} \ldots \ldots \ldots$ Set of all measurable selections of the $\alpha$-cut of a fuzzy probability density $f^{\star}$ which are classical probability densities too

$\mathcal{D}_{\alpha} \ldots \ldots \ldots$. Set of all functions (classes) in $L_{1}$, which are measurable selections of the $\alpha$-cut of a fuzzy probability density $f^{\star}$ and classical probability densities too

$\delta_{H}(\cdot, \cdot) \ldots \ldots$ Hausdorff metric

$\delta_{H, \infty}^{\star}(\cdot, \cdot) \ldots \ldots$ Uniform metric induced by the Hausdorff metric $\delta_{H}$

$\delta_{H, p}^{\star}(\cdot, \cdot) \ldots \ldots \ldots L^{p}$-metric induced by the Hausdorff metric $\delta_{H}$

$\mathbb{E}(X) \ldots \ldots \ldots$ Expectation of the random variable $X$

$f^{\star}(\cdot) \ldots \ldots \ldots$ Fuzzy probability density

$\bar{f}_{\alpha}(\cdot) \ldots \ldots \ldots$ Upper $\alpha$-level function of a fuzzy probability density $f^{\star}$

$\underline{f}_{\alpha}(\cdot) \ldots \ldots \ldots$. Lower $\alpha$-level function of a fuzzy probability density $f^{\star}$

$\mathcal{F}_{c}^{1} \ldots \ldots \ldots \ldots$ Set of all fuzzy numbers

$\mathcal{F}_{c, c}^{1} \ldots \ldots \ldots \ldots$ Set of all fuzzy numbers with compact support

$\mathcal{F}_{c}^{d} \ldots \ldots \ldots \ldots$ Set of all $d$-dimensional fuzzy vectors

$\mathcal{F}_{c, c}^{d} \ldots \ldots \ldots \ldots$ Set of all $d$-dimensional fuzzy vectors with compact support

$\mathcal{F}_{c, p}^{d} \ldots \ldots \ldots \ldots$ Set of all fuzzy vectors with $p$-integrable support functions on $S^{d-1} \times(0,1]$ with respect to the product measure $\vartheta \otimes \lambda$

$\Gamma\left(X_{\alpha}\right) \ldots \ldots \ldots$ Graph of $X_{\alpha}$

$\mathcal{G}_{\text {sous }}(\cdot) \ldots \ldots$ Generated family of Souslin sets

$h_{n}(B, \omega) \ldots \ldots$. Classical relative frequency of the set $B$ with respect to realizations $X_{1}(\omega), \cdots, X_{n}(\omega)$

$h_{n}^{\star}(B) \ldots \ldots$. Fuzzy relative frequency of the set $B$ given a fuzzy sample $x_{1}^{\star}, x_{2}^{\star}, \ldots, x_{n}^{\star}$ 
$\bar{h}_{n, \alpha}(B) \ldots \ldots$ Upper relative frequency of level $\alpha$ of the set $B$ given a fuzzy sample $x_{1}^{\star}, x_{2}^{\star}, \ldots, x_{n}^{\star}$

$\underline{h}_{n, \alpha}(B) \ldots \ldots$. Lower relative frequency of level $\alpha$ of the set $B$ given a fuzzy sample $x_{1}^{\star}, x_{2}^{\star}, \ldots, x_{n}^{\star}$

$h_{n}^{\star}(B, \omega) \ldots \ldots$ Fuzzy relative frequency of the set $B$ given realizations $X_{1}^{\star}(\omega), X_{2}^{\star}(\omega), \ldots, X_{n}^{\star}(\omega)$

$\bar{h}_{n, \alpha}(B, \omega) \ldots$ Upper relative frequency of level $\alpha$ of the set $B$ given realizations $X_{1}^{\star}(\omega), X_{2}^{\star}(\omega), \ldots, X_{n}^{\star}(\omega)$

$\underline{h}_{n, \alpha}(B, \omega) \ldots$ Lower relative frequency of level $\alpha$ of the set $B$ given realizations $X_{1}^{\star}(\omega), X_{2}^{\star}(\omega), \ldots, X_{n}^{\star}(\omega)$

inf ............ Infimum

$\operatorname{int}(A) \ldots \ldots$. Interior of the set $A$

$\mathcal{K}^{d} \ldots \ldots \ldots \ldots$ Set of all non-empty compact sets in $\mathbb{R}^{d}$

$\mathcal{K}_{c}^{d} \ldots \ldots \ldots \ldots$ Set of all non-empty compact, convex sets in $\mathbb{R}^{d}$

$\lambda \ldots \ldots \ldots \ldots$. Lebesgue measure

$\lim \ldots \ldots \ldots \ldots$ Limes

limsup $\ldots \ldots \ldots$ Limes superior

$\lim \inf \ldots \ldots \ldots$. Limes inferior

$\mathcal{L}^{p}(\Omega, \mathcal{A}, \mu) \ldots$ set of all $\mathcal{A}$-measurable real-valued functions $f$, such that $|f|^{p}$ is integrable on $\Omega$ with respect to the measure $\mu$

$L^{p}(\Omega, \mathcal{A}, \mu) \ldots$ Banach space of all equivalence classes of $p$-integrable realvalued functions on $\Omega$

$\rho_{p}(\cdot, \cdot) \ldots \ldots \ldots L^{p}$-distance induced by support functions

$\rho_{p}^{\star}(\cdot, \cdot) \ldots \ldots \ldots L^{p}$-metric for fuzzy vectors induced by the $L^{p}$-metric $\rho_{p}(\cdot, \cdot)$

$\max \ldots \ldots \ldots$ Maximum

$m_{1}^{\star} \ldots \ldots \ldots \ldots$ Expectation of a fuzzy probability distribution $\mathbb{P}^{\star}$ induced by a fuzzy probability density $f^{\star}$

$\min \ldots \ldots \ldots$ Minimum

$m_{k}^{\star} \ldots \ldots \ldots \ldots, k$-th moment of a fuzzy probability distribution $\mathbb{P}^{\star}$ induced by a fuzzy probability density $f^{\star}$

$\tilde{m}_{k}^{\star} \ldots \ldots \ldots \ldots k$-th central moment of a discrete fuzzy probability distribution $\mathbb{P}^{\star}$

$\mu_{1} \otimes \mu_{2} \ldots \ldots$. Product measure of $\mu_{1}$ and $\mu_{2}$

$(\Omega, \mathcal{A}, \mathcal{P}) \ldots \ldots$ Probability space

$\bar{p}_{\alpha}(\cdot) \ldots \ldots \ldots$. Upper boundary of the $\alpha$-cut of a fuzzy probability distribution $\mathbb{P}^{\star}(\cdot)$

$\underline{p}_{\alpha}(\cdot) \ldots \ldots \ldots$ Lower boundary of the $\alpha$-cut of a fuzzy probability distribution $\mathbb{P}^{\star}(\cdot)$ 
$\mathcal{P} \ldots \ldots \ldots$. . Probability measure

$\mathbb{P}^{\star}(\cdot) \ldots \ldots \ldots$ Fuzzy probability distribution

$\operatorname{Sel}(\mathrm{F}) \ldots \ldots \ldots$ Set of all measurable selections of a set-valued function $F$

SLLN ......... Strong Law of Large Numbers

$S^{d-1} \ldots \ldots \ldots d$-dimensional unit sphere

$\operatorname{supp}\left(\xi^{\star}\right) \ldots \ldots$ Support of a fuzzy number or fuzzy vector

$s_{A}(\cdot) \ldots \ldots \ldots$ Support function of the set $A$

$s_{A^{\star}}(\cdot, \cdot) \ldots \ldots$. Support function of a fuzzy number or fuzzy vector $A^{\star}$

$\sup \ldots \ldots \ldots$...... Supremum

$\vartheta \ldots \ldots \ldots$ Normalized Lebesgue (surface) measure on $S^{d-1}$

$T \ldots \ldots \ldots$. Time for fuzzy stochastic processes

$x_{1}, x_{2}, \ldots, x_{n} \ldots$ Real-valued sample of size $n$

$x_{1}^{\star}, x_{2}^{\star}, \ldots, x_{n}^{\star} \ldots$ Fuzzy sample of size $n$

$X^{\star}(\cdot) \ldots \ldots \ldots$. Fuzzy random variable or fuzzy random vector

$X_{\alpha}(\cdot) \ldots \ldots \ldots \ldots$-cut of a fuzzy random variable or fuzzy random vector $X^{\star}$

$\bar{X}_{\alpha}(\cdot) \ldots \ldots \ldots$ Upper boundary of the $\alpha$-cut of a fuzzy random variable $X^{\star}$

$\underline{X}_{\alpha}(\cdot) \ldots \ldots \ldots$ Lower boundary of the $\alpha$-cut of a fuzzy random variable $X^{\star}$

$\left(X_{t}^{\star}\right)_{t \in T} \ldots \ldots \ldots$ Fuzzy stochastic process 


\section{Bibliography}

[1] M.F. Barnsley: Fractals everywhere, Academic Press, Cambridge, 1993

[2] H. Bauer: Maß- und Integrationstheorie, W. de Gruyter Verlag, Berlin New York, 1993

[3] H. Bauer: Wahrscheinlichkeitstheorie, W. de Gruyter Verlag, Berlin New York, 2002

[4] D. Bierlein: Der Graph meßbarer Funktionen mit abstraktem Definitionsbereich, Math. Zeitschrift 76, 468-471 (1961)

[5] J.J. Buckley: Graphs of measurable functions, Proc. Amer. Math. Soc. 44, 78-80 (1974)

[6] J.J. Buckley: Fuzzy Probabilities, Physica, Heidelberg New York, 2003

[7] C. Castaing, M. Valadier: Convex Analysis and Measurable Multifunctions, Lecture Notes in Mathematics, Springer, Berlin Heidelberg New York, 1977

[8] G. Choquet: Theory of capacities, Ann. Inst. Fourier 5, 131-295 $(1953 / 54)$

[9] P. Diamond, P. Kloeden: Metric Spaces of Fuzzy Sets, World Scientific, Singapore, 1994

[10] P. Diamond, P. Kloeden: Metric spaces of fuzzy sets, Fuzzy Sets and Systems 35, 241-249 (1990)

[11] J.L. Doob: Stochastic Processes, John Wiley \& Sons, New York, 1953

[12] N. Dunford, J.T. Schwartz: Linear Operators, Wiley-Interscience, New York, 1958

[13] J. Elstrodt: Maß- und Integrationstheorie, Springer Verlag, Berlin Heidelberg New York, 1999 
[14] N. Etemadi: An elementary proof of the strong law of large numbers, Zeitschrift für Wahrscheinlichkeitstheorie und verwandte Gebiete $\mathbf{5 5}$ 119-122 (1981)

[15] D. Hareter:Zeitreihenanalyse mit unscharfen Daten, Dissertation, TU Wien, 2003

[16] E. Hewitt, K. Stromberg: Real and Abstract Analysis, Springer Verlag, Berlin Heidelberg, 1965

[17] B.K. Kim, J.H. Kim: Stochastic Integrals of Set-Valued Processes and Fuzzy Processes, Journal of Mathematical Analysis and Applications 236, 480-502 (1999)

[18] E.P. Klement, M.L. Puri, D.A. Ralescu: Limit theorems for fuzzy random variables, Proc. Roy. Soc. London, A 407, 171-182 (1986)

[19] R. Körner: Linear Models with Random Fuzzy Variables, Dissertation der TU Bergakademie Freiberg, 1997

[20] V. Krätschmer: Some complete metrics on spaces of fuzzy subsets, Fuzzy Sets and Systems 130, 357-365 (2002)

[21] H. Kwakernaak: Fuzzy random variables - I. Definitions and theorems, Inform. Sci. 15, 1-29 (1978)

[22] H. Kwakernaak: Fuzzy random variables - II. Algorithms and examples for the discrete case, Inform. Sci. 17, 253-278 (1979)

[23] A. Lasota, M. C. Mackey: Chaos, Fractals and Noise, Springer Verlag, New York, 1994

[24] M. Ledoux, M. Talagrand: Probability in Banach Spaces, Springer Verlag, Berlin Heidelberg New York, 1991

[25] J. Lehn: Prämeßbare Funktionen, manuscripta math. 20, 141-152 (1977)

[26] J. Lehn: Remark on measurable graph theorems, Proc. Amer. Math. Soc. 63, 46-48 (1977)

[27] G. Matheron: Random Sets and Integral Geometry, Wiley, New York, 1975

[28] R.E. Megginson: An Introduction to Banach Space Theory, Springer, New York, 1998 
[29] I. Molchanov: Theory of Random Sets, Springer, London, 2005

[30] B. Möller, M. Beer: Fuzzy Randomness - Uncertainty in Civil Engineering and Computational Mechanics, Springer, Berlin, 2004

[31] B. Möller, M. Beer, U. Reuter: Theoretical Basis of Fuzzy Randomness - Application to Time Series with Fuzzy Data, in: G. Augusti, G.I. Schuëller, M. Ciampoli (Eds.): Safety and Reliability of Engineering Systems and Structures - Proceedings of the 9-th International Conference on Structural Safety and Reliability, Millpress, Rotterdam, pp. 1701 - 1707, 2005

[32] S. Niculescu, R. Viertl: Bernoulli's Law of Large Numbers for Vague Data, Fuzzy Sets and Systems 50, 167-173 (1992)

[33] M.L. Puri, D.A. Ralescu: Fuzzy random variables, J. Math. Anal. Appl. $114,409-422(1986)$

[34] H. Radström: An embedding theorem for spaces of convex sets, Proc. Amer. Math. Soc. 3, 165-169 (1968)

[35] W. Rudin: Real and Complex Analysis, McGraw-Hill International Editions, Singapore, 1987

[36] W. Rudin: Functional Analysis, McGraw-Hill International Editions, Singapore, 1991

[37] R. Schneider: Convex Bodies: The Brunn-Minkowski Theory, Cambridge University Press, 1993

[38] S.M. Srivastava: A Course on Borel Sets, Springer Verlag, New York, 1998

[39] W. Trutschnig: Iterierte Funktionensysteme und Chaos Game, Diplomarbeit, TU Wien, 2001

[40] W. Trutschnig, D. Hareter: Fuzzy Probability Distributions, in: M. Lopez-Diaz, M.A. Gil, P. Grzegorzewski, O. Hryniewicz, J. Lawry (Eds.): Soft Methodology and Random Information Systems, SpringerVerlag, Heidelberg, pp. 399 - 406, 2004

[41] R. Viertl, D. Hareter: Fuzzy Information and Imprecise Probability, ZAMM 84, No. 10-11 (2004)

[42] R. Viertl, D. Hareter: Beschreibung und Analyse unscharfer Information, Springer, Wien New York, 2006 
[43] R.A. Vitale: $L_{p}$ Metrics for Compact, Convex Sets, Journal of Approximation Theory 45, 280-287 (1985)

[44] G. Wang, Y. Zhang: The theory of fuzzy stochastic processes, Fuzzy Sets and Systems 51, 161-178 (1992)

[45] Z. Wang, G. Klir: Fuzzy Measure Theory, Plenum Press, New York, 1992 
Wolfgang Trutschnig

Quellenstraße 160/12

1100 Wien

trutschnig@statistik.tuwien.ac.at

\title{
Curriculum Vitae
}

\author{
Geburtsdatum: $\quad$ 14.01.1977 \\ Geburtsort: Lienz (Osttirol), Österreich \\ Staatsangehörigkeit: Österreich \\ Familienstand: ledig
}

\section{Ausbildung:}

1991-1995: $\quad$ BORG Lienz (naturwissenschaftliche Richtung)

Matura mit Ausgezeichnetem Erfolg

1995-2001: $\quad$ Studium der Technischen Mathematik an der TU Wien, Studienzweig Mathematik in den Naturwissenschaften Diplomarbeit 'Iterierte Funktionensysteme und Chaos Game' 2001 Abschluss mit Ausgezeichnetem Erfolg

11/2001-8/2003: Wissenschaftlicher Mitarbeiter am Mathematikinstitut der Universität Wien, Forschungsgruppe Ergodentheorie

seit 9/2003: $\quad$ Dissertant bei Prof. Viertl an der TU Wien

Thema: 'Fuzzy Probability Distributions'

\section{Arbeitserfahrung:}

9/2003-1/2004: Beschäftigt bei der Firma Drei (Hutchison) im Bereich Regulatory Affairs

2/2004-12/2005: Beschäftigt bei der Oesterreichischen Nationalbank in der Abteilung für Zahlungsbilanzangelegenheiten, Hauptabteilung Statistik 


\section{Lehre:}

1997: $\quad$ Übungsgruppenleiter Mathematik für

Lebensmitteltechnologen an der BOKU Wien

1997-2000: $\quad$ Leiter von Mathematikkursen am BFI Tirol und in diversen Nachhilfeinstituten in Wien

Seit 1998: $\quad$ Übungsgruppenleiter Mathematik 1-3 für Elektrotechniker an der TU Wien

WS 2004: $\quad$ Übungsgruppenleiter für Maß- und

Wahrscheinlichkeitstheorie an der TU Wien

\section{Stipendien:}

1996-1999, 2002: Leistungsstipendium der TU Wien 